\title{
Exploring the Past, Present, and Future of Cultural Competency Research: The Revision and Expansion of the Sociocultural Adaptation Construct
}

\author{
Jessie Kaye Wilson \\ A thesis submitted to Victoria University of Wellington \\ in fulfilment of the requirements for the degree of \\ Doctor of Philosophy in Psychology
}

Victoria University of Wellingon 


\begin{abstract}
International migration trends have heralded a marked increase in intercultural contact, creating a greater need for effective cultural competency in both inter- and intra-cultural situations. The current research programme, positioned within the field of acculturation psychology, examined a specific behavioural aspect of cultural competency known as sociocultural adaptation. Defined as an individual's acquisition and expression of culturally appropriate behavioural skills used to negotiate interactive aspects of a new cultural setting, an in-depth examination of the sociocultural adaptation construct was provided. Three studies addressed issues concerning the review, revision, and expansion of work on the topic of cross-cultural behavioural competency. Study 1 offered a meta-analytic review of the correlates or antecedents of sociocultural adaptation. Results emphasised the importance of individual differences, such as personality characteristics and motivation, in relation to adaptation difficulties. Suggestions were also provided for future theoretical and applied research regarding how demographic (e.g, age, gender), situational (e.g., language proficiency), and individual differences (e.g., cross-cultural empathy) components relate to and influence an individual's successful cross-cultural adjustment. Study 2 examined the operationalisation of behavioural competency through revision of an existing measure of sociocultural adaptation (the Sociocultural Adaptation Scale or SCAS) and investigated five adjustment domains: Ecological, interpersonal, personal interests and community involvement, language, and professional/work adjustment. The final study sought to corroborate the factor structure of the revised SCAS and explored the effects of migration motivation and perceived discrimination-two underrepresented variables in the acculturation literature-in relation to cross-cultural adjustment using path analysis techniques. Direct linkages were found between migration motivation and positive psychological outcomes, and behavioural competency and discrimination were found to have significant mediating effects on the relationship between these two variables. The limitations and contributions of these studies are discussed in relation to the existing acculturation psychology literature, and new avenues for theoretical and applied applications of the findings are suggested.
\end{abstract}




\section{Acknowledgements}

"The more that you read, the more things you will know. The more that you learn, the more places you'll go.” (Dr. Seuss, 1978, p. 29)

In February of 2008 I stuffed my life into two big suitcases and boarded a plane to Wellington to begin my doctoral studies. Little did I know that my academic journey would also involve me calling New Zealand home with a resident visa, cat, husband, and six-month old daughter, too! Along this winding $\mathrm{PhD}$ road I've read more than I believed possible, know even less than I thought I did before, and have several people to thank for helping me reach the place where I find myself today.

First and foremost, I wouldn't have gotten on that plane if it weren't for Education New Zealand and Victoria University. I am extremely thankful for the financial support both organisations have provided me throughout my academic endeavours. And thank you to those who participated in my research: Your time and effort has created the backbone of this thesis! I am also grateful to those at the Department of Labour, the Office of Ethnic Affairs, and the various embassies and consulates who helped to distribute survey materials.

I have met an amazing group of people during my studies at Victoria who have encouraged me to keep walking toward the finish line. To my primary supervisor Colleen: Thank you so much for your wisdom, patience, grammar lessons, and wine. There are two sides to the research story, and you have given me a better understanding of both. Thank you also to my secondary supervisor Ron for his contributions. Furthermore, the Centre for Applied Cross-Cultural Research and its members-past and present-deserve mention. Thanks particularly to Floor, Aidan, and Jai for their assistance with data collection and the literature review. A big thank you to my examiners: Your suggestions have made this thesis a better, stronger piece of writing. And to the School of Psychology staff-Ngaire, Nic, Wendy, and Jebi-thank you for supporting me and so many others in our scholastic undertakings.

There are others who helped make this $\mathrm{PhD}$ a reality and have also made New Zealand my second home. Jaimee, it may be that your heart is too big sometimes, but I am the lucky recipient all the same of the generosity and kindness it holds. Let's keep thinking big together. Robyn, I couldn't have asked for a better PhD ally, and I am honoured to call both you and George friends. You inspire me to be good and do better. Thanks to Jarrod for 
proofreading, and also to Wendy and Pip. Your encouragement and friendship means so much to me.

A big thank you to my friends back in the States. David, you encouraged me to take that first step into the world of psychology. I will always be appreciative for that. To Ray Sunshine Leki, as promised. And to Maria and Jess, who are also journeying down this precarious $\mathrm{PhD}$ path. We have traversed a good many miles together already, girls, with many more adventures to come.

Many thanks to my absolutely amazing Kiwi family: I couldn't have asked for a more spirited and intelligent whānau to become a part of. You have kept me going with all of those pizza nights and knitting sessions! Thank you especially to Robyn, who has time and again demonstrated a supernatural ability to be a wonderful mentor, mum-in-law, grandmother, friend, and fellow $\mathrm{PhD}$ candidate... simultaneously.

To the Bird House inhabitants on Bird St near Sank Park. Da ville may not be a tropical paradise, but you, Mam, Dah, and my seester Kowz, provide an island sanctuary nonetheless. The three of you have always nurtured my interests-academic and extracurricular - and are the best fan club anyone could ever hope for. Thank you for catching those rambling sentences, keeping me laughing, and for your support and love. ILU!

Playing the most important support role in the completion of this dissertation is my fabulous husband Simon. You have been there for me from when this research was nothing more than primordial soup, and have had a bigger hand in its evolution than I can chronicle (but I could start with your words of encouragement, counselling, house cleans, dinners, baby-minding, shoulder massages, and tech support). I appreciate and love you to the moon and back!

Last, to my smiling daughter Quinn Celia Tuohy, the true PhD baby. Thank you for the new perspective you've brought to my life, and for not crawling until after I submit. Here's to all of your future reading, learning, knowing, and going! 


\section{Table of Contents}

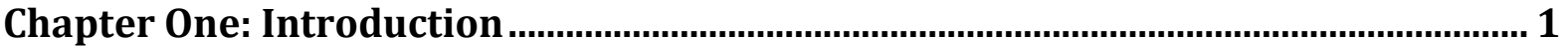

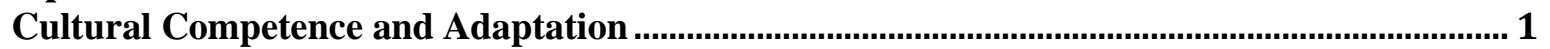

Acculturation and Adaptation: Theoretical Frameworks......................................................... 3

Inside the Acculturative Framework: The Culture Learning Paradigm .......................................... 7

Advancement of the Culture Learning Paradigm .........................................................................12

Adaptation within the Behavioural Domain of Cultural Competency......................................14

Measurement of Sociocultural Adaptation .......................................................................

The Current Research Programme ......................................................................................

Chapter Two: A Meta-Analysis of the Correlates of Sociocultural Adaptation ........21

Review of Sociocultural Adaptation and the SCAS .................................................................22

Culture Learning Theory: Situational Factors and Sociocultural Adaptation..........................22

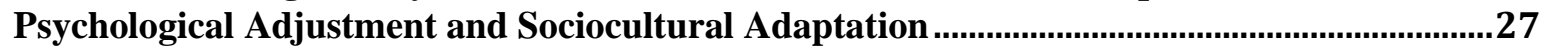

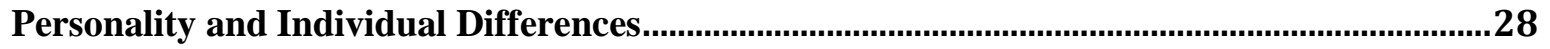

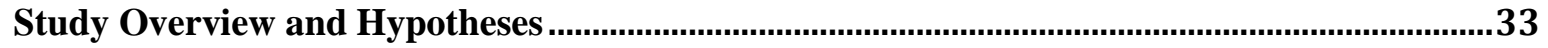

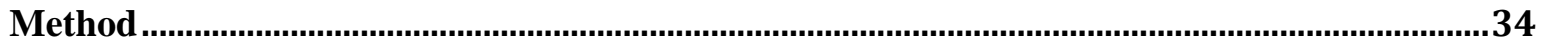

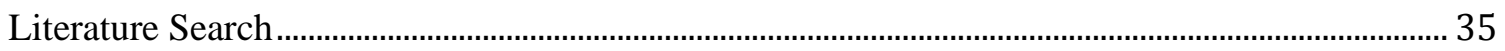

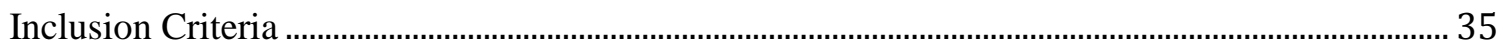

Coding and Meta-Analytic Procedures............................................................................................... 36

Homogeneity Analysis and Moderator Analyses .............................................................................. 37

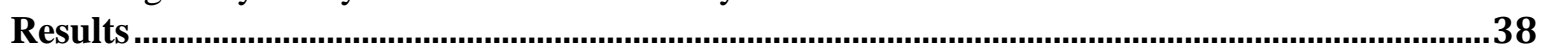

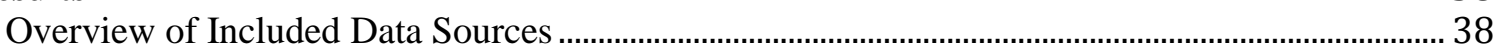

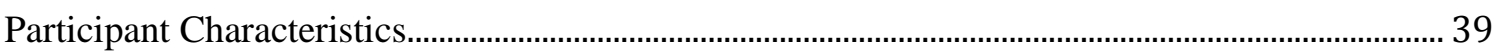

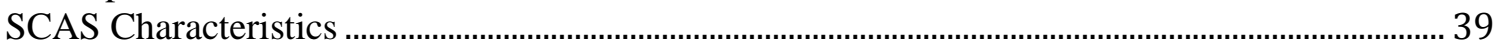

Demographic, Situational, Psychological Adjustment, and Individual Differences Correlates of

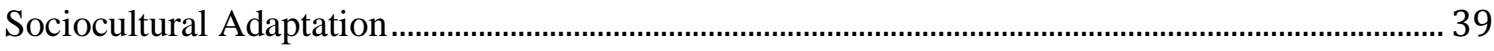

Moderator Analyses Results...................................................................................................... 41

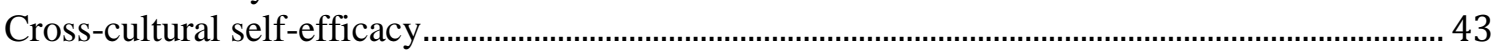

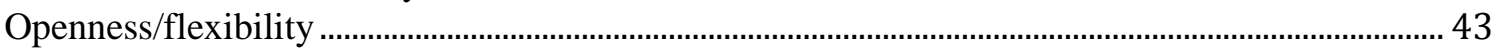

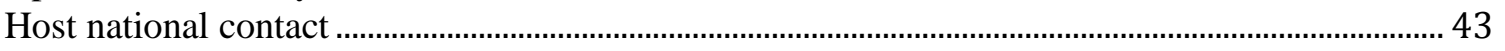

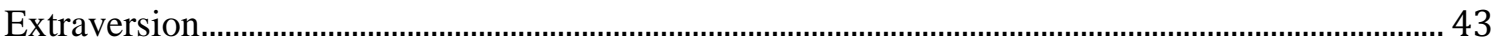

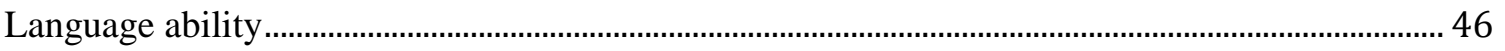

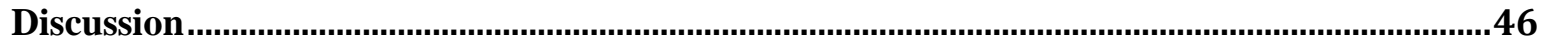

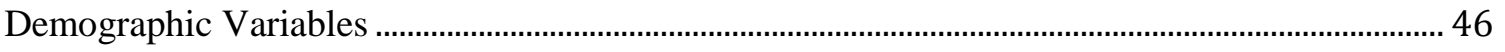

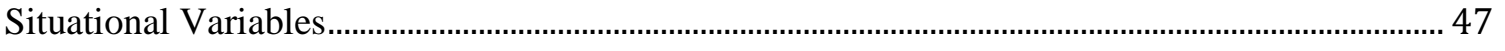

Psychological Adjustment................................................................................................................ 49

Individual Differences: Personality and Motivation Factors ................................................................ 51

Broad personality and motivation factors …………........................................................................ 51

Specific personality and motivation variables .................................................................................... 53

Meta-analytic Contributions to Acculturation Theory and Research................................................. 54

Limitations, Conclusions, and Future Directions …………………………………………………....... 56

Chapter Three: Development and Validation of a Revised Measure of Sociocultural

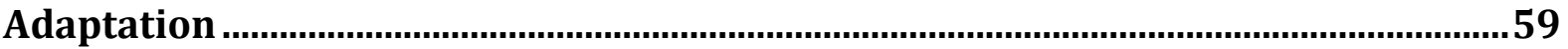

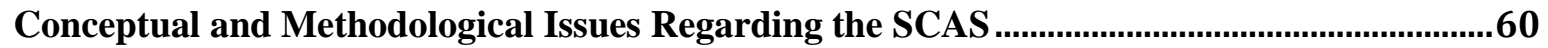

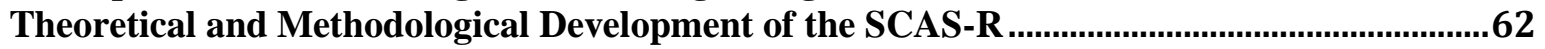

Theoretical development of the SCAS-R: Domain generation ............................................................... 63

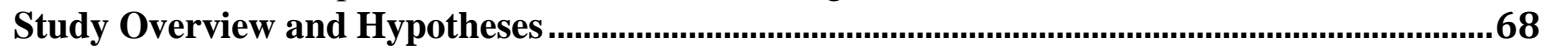

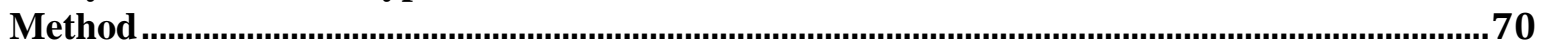

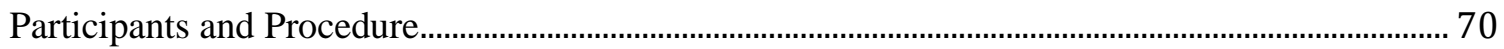

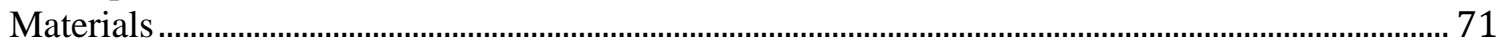




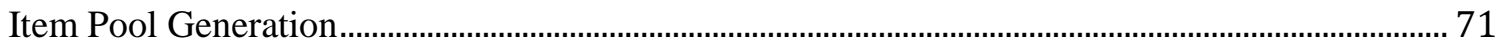

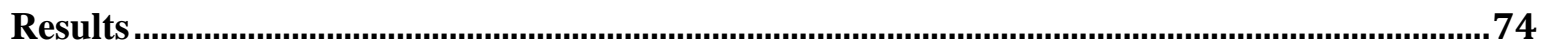

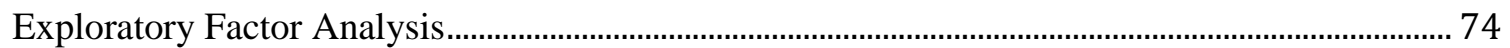

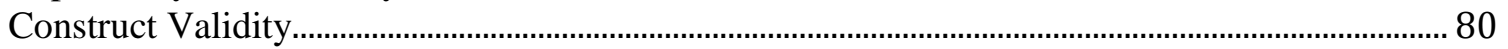

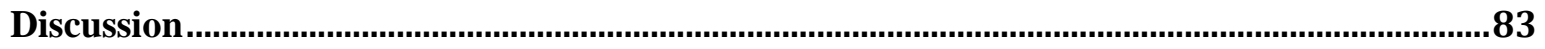

Exploratory Factor Analysis, Internal Reliability, Construct Validity of the SCAS-R, and General

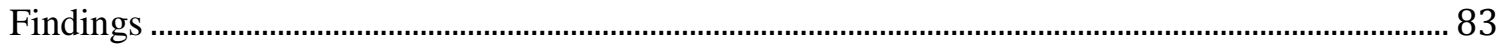

Limitations and Future Directions........................................................................................................... 86

Chapter Four: Migration Motivation, Cultural Competence, and Discrimination:

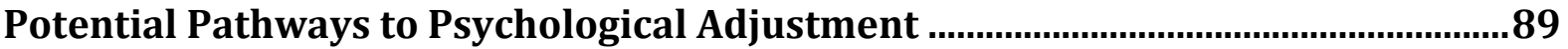

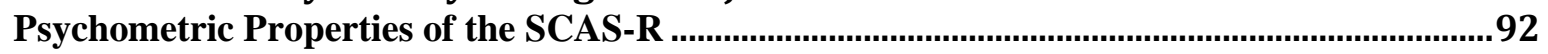

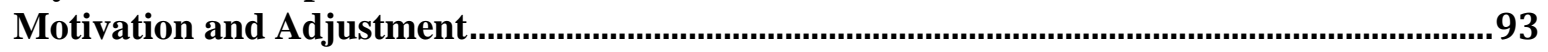

Migration motivation and adjustment outcomes................................................................................. 95

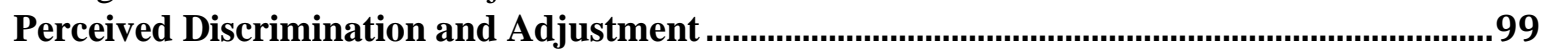

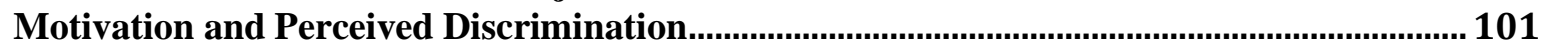

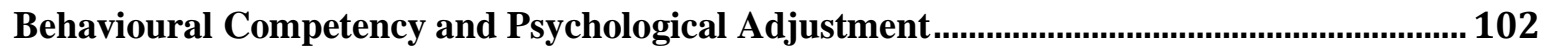

The Potential Mediating Effects of Perceived Discrimination ...................................................... 103

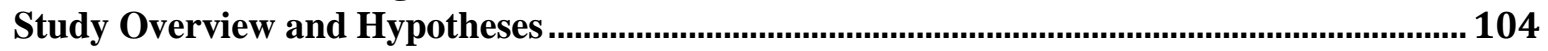

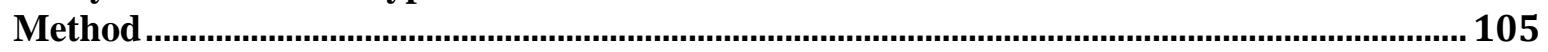

Procedure

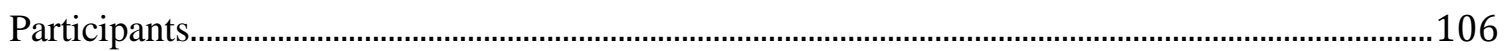

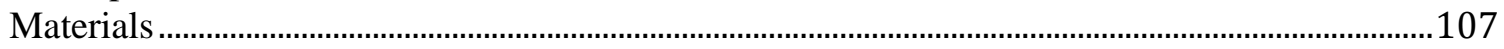

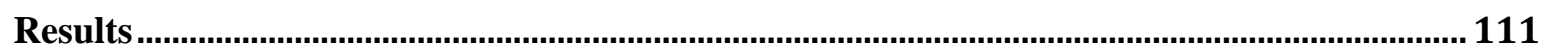

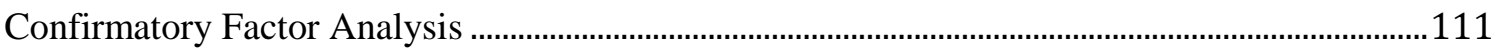

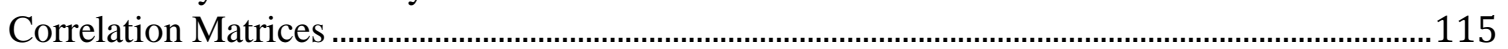

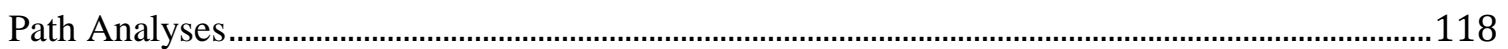

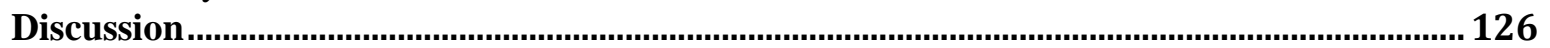

Confirmatory Factor Analysis and Internal Reliability of the SCAS-R ..........................................126

Pathways between Migration Factors and Adjustment.......................................................................130

Pathways between Migration Motivation and Adjustment...............................................................132

Pathways between Perceived Discrimination and Psychological Adjustment .................................133

Pathways between Behavioural Competency, Perceived Discrimination, and Psychological

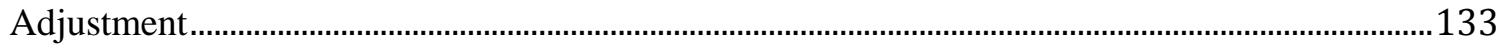

Indirect Pathways: The Mediating Effects of Competency and Perceived Discrimination .........134

Limitations and Future Directions........................................................................................................

Chapter Five: General Discussion.......................................................................... 139

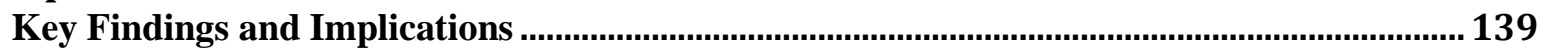

Beyond Culture Learning Theory: A New Look at Sociocultural Adaptation Correlates............139

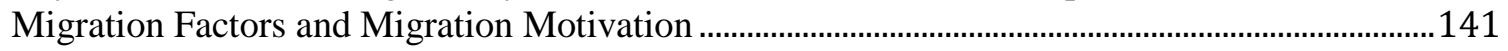

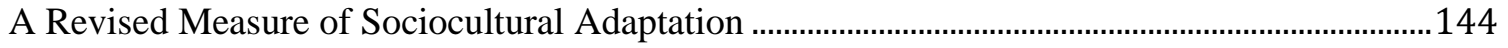

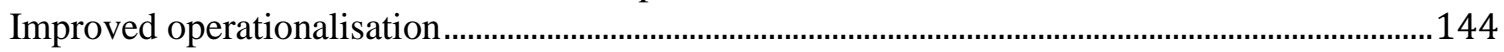

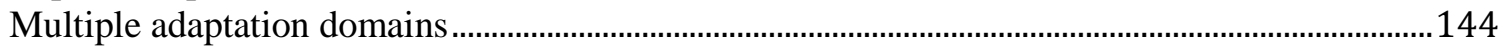

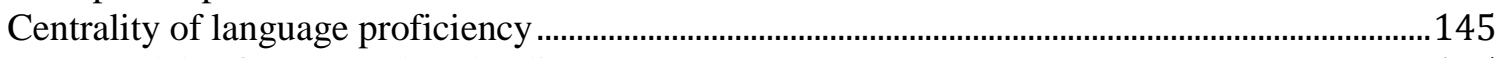

New Models of Cross-Cultural Adjustment ................................................................................. 145

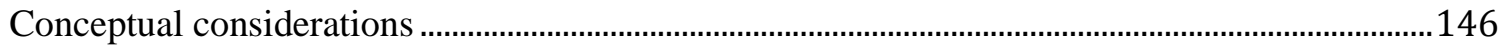

Methodological considerations....................................................................................................146

Refining the Model and Measurement of Acculturation...................................................................147

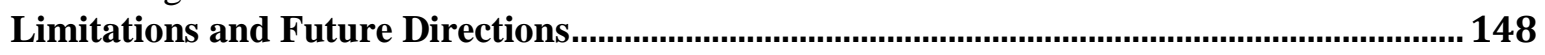

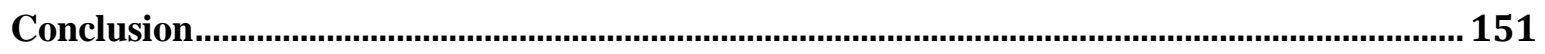

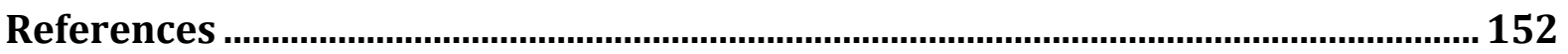

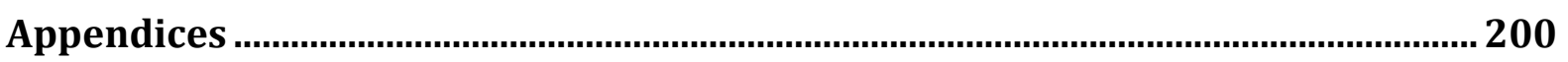


Appendix A. Study Characteristics of the Meta-Analysis ....................................................................20

Appendix B. Overview of Multi-National Studies..............................................................................208

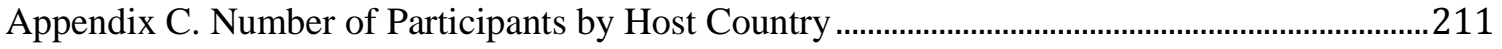

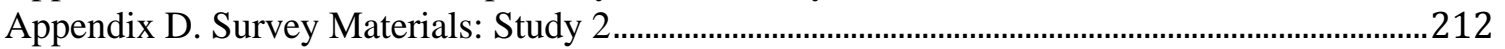

Appendix E. Study 3 Participant Demographics ...........................................................................228

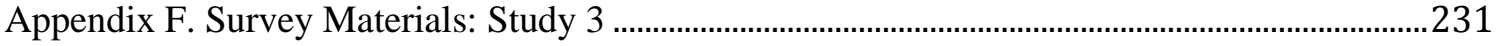




\section{List of Tables}

Table 1. Effect Sizes per SCAS Correlate Based on the Fixed-Effects Model ............... 40

Table 2. $\quad$ ANOVA Summary for the Presence of Moderator Variables ........................... 44

Table 3. Results of Variance Explained from 21-item SCAS-R .................................... 76

Table 4. SCAS-R Factor Loadings for Exploratory Factor Analysis with Oblique

Promax Rotation …........................................................................................ 77

Table 5. Internal Consistency, Means, and Standard Deviations of the SCAS-R and

SCAS-R Subscales

Table 6. $\quad$ Correlations Between SCAS-R and Other Adjustment Indices........................ 81

Table 7. Correlations Between SCAS-R Subscales and Other Adjustment Indices

Table 8. Means, Standard Deviations, and Zero-Order Correlations Among

Variables

Table 9. Confirmatory Factor Analysis of the SCAS-R and Factor Loadings

of Items

Table 10. Means, Standard Deviations, and Zero-Order Correlations Among

SCAS-R Subscales and Study Variables

Table 11. $\quad$ Summary of the Direct Paths Between Migration Factors and

Psychological Adjustment Outcomes

Table 12. Summary of the Indirect Paths of Sociocultural Adaptation and

Perceived Discrimination on the Relationship Between Lifestyle Goals

and Psychological Adjustment Outcomes.

Table 13. $\quad$ Summary of the Direct Paths Between Migration Motivation and

Psychological Adjustment.

Table 14. Summary of the Indirect Paths of Sociocultural Adaptation and

Perceived Discrimination on the Relationship Between Migration

Motivation and Psychological Adjustment 


\section{List of Figures}

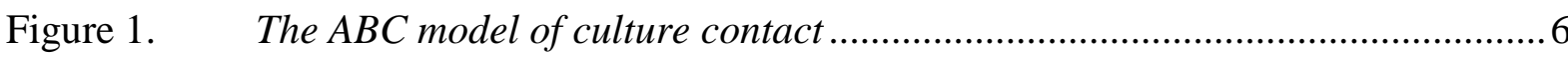

Figure 2. Interactive model of foreign language proficiency, communication competence, effective intercultural interaction and sociocultural adaptation 64

Figure 3. Results of scree plot from the 21-item SCAS-R .76

Figure 4. Hypothesised path model illustrating the mediating effects of sociocultural adaptation and perceived discrimination on the relationship between migration factors and psychological adjustment outcomes....

Figure 5. Hypothesised path model illustrating the mediating effects of sociocultural adaptation and perceived discrimination on the relationship between migration motivation and psychological adjustment outcomes. 92

Figure 6. Hypothesised first-order CFA model of the SCAS-R

Figure 7. Re-specified first-order CFA model of the SCAS-R

Figure 8. Refitted path model illustrating the mediated effects of sociocultural adaptation and perceived discrimination on the relationship between lifestyle goals and psychological adjustment outcomes.

Figure 9. Refitted path model illustrating the mediated effects of sociocultural adaptation and perceived discrimination on the relationship between migration motivation and psychological adjustment.

Figure 10. Hypothesised pathways tested in Study 3 between the frameworks, processes, and outcomes of the ABC model of culture contact

Figure 11. Adapted ABC model of culture contact. 


\section{Chapter One: Introduction}

\section{Cultural Competence and Adaptation}

In our world today, people are moving across international boundaries at everincreasing and unprecedented rates. Approximately one out of every 33 people in the world is a migrant, with over 214 million people estimated to be living abroad in 2010; an increase from 194 million in 2005 and 150 million in 2000 (UNDESA, 2011). There are at least 38 million expatriates living in the United States alone, and 15.4 million refugees and 3.4 million international students worldwide (Ward, 2012). These international migration trends have heralded a marked increase in intercultural contact. This contact is occurring both across countries in terms of the cross-cultural adaptation of individuals and groups, as well as within countries with regard to intercultural relations and the political, organisational, and social management of cultural diversity. With this global rise in cross-cultural contact and cultural diversity, there has never before been a greater demand for competence in withinculture interactions and across-culture transitions.

What constitutes intercultural effectiveness or cultural competence is, however, dependent upon definition, conceptualisation, and interpretation. Cultural competence within national boundaries (e.g., cultural diversity and intercultural relations) has been conceptualised in fields such as educational, clinical and counselling psychology as successful therapeutic interactions with multicultural clientele, cultural sensitivity, and the development of programmes containing multicultural content (Arthur \& Achenbach, 2002; Chao, Okazaki, \& Hong, 2011; Pedersen, Draguns, Lonner, \& Trimble, 2007; Sue, Arrondondo, \& McDavies, 1992). In organisational contexts, on the other hand, cultural competency may be associated with expatriate retention, productive multicultural work teams, management of culturally diverse groups to obtain organisational goals, or specific task or job performance (Mol, Born, \& van der Molen, 2005; Pedersen, Draguns, Lonner, \& Trimble, 2007; Sue, Arrondondo, \& McDavies, 1992; Tan \& Chua, 2003).

Alternatively, cultural competence across national boundaries (e.g., cross-cultural transition) can be described at the most general level as an individual's potential ability to be effective in a new cultural environment (Gertsen, 1990). Three broad aspects of intercultural competence exist: (1) A cognitive component that involves integration of knowledge and perceptions about a new culture; (2) a skill element that entails behaviours acquired in a new setting; and (3) an intrapersonal component comprised of attitudes, personality traits, and 
motivations towards new situations that enables cross-cultural effectiveness (Hannigan, 1990).

A variety of ways exist to measure effective cross-cultural adjustment outcomes within these three cognitive, skill, and intrapersonal domains of cultural competency. According to a review provided by Ward and Kennedy (1999), for example, successful crosscultural outcomes can be relational in nature, such as an individual's perceptions and feelings of acceptance from peers and the host society (Brislin, 1981; Deshpande \& Viswesvaran, 1992; Eshel \& Rosenthal-Sokolov, 2000) or frequency and/or satisfaction with contact between hosts and co-nationals (Bakker, Van Oudenhouven, \& Van der Zee, 2004; Berry, Phinney, Sam, \& Vedder, 2006). Identity indices of cross-cultural adjustment have also been utilised, which involve conflict with and/or management of cultural and host identities (Leong \& Ward, 2000; Ward, Stuart, \& Kus, 2011). Other outcome indices have included health measures of medical or physical symptoms (Anderzen \& Arnetz, 1999; Babiker, Cox, \& Miller, 1980; Dasen, Berry, \& Sartorius, 1988); task-specific outcomes such as employment or academic performance (Aycan, 2008; Berry, Phinney, Sam, \& Vedder, 2006; Black, 1988; Black \& Stephens, 1989; Leung, 2001); psychological measures of sociocultural distress or concern (Rohrlich \& Martin, 1991; Schupe, 2007), life satisfaction (Sam, 2001; Ullman \& Tatar, 2001) and depression (Aronowitz, 1984; Berry \& Kim, 1988; Torres \& Rollock, 2007); and behavioural indices that assess cultural skills acquisition (Bochner, McLeod, \& Lin, 1977; Hammer, 1987; Harrison, Chadwick, \& Scales, 1996; Kosic, 2004; Ruben, 1989; Sodowsky \& Lai, 1997; Torbiorn, 1982).

Some researchers have taken a more methodical approach to the theoretical and applied examination of cultural competence and effective cross-cultural transitions. For instance, Hammer, Gudykunst, and Wiseman (1978) conducted a series of factor analytic studies that identified stress management, relational, and communication aspects of intercultural effectiveness. Black, Mendenhall, and Oddou (1991) have also initiated a large body of research integrating various theories on successful work, interaction, and general adjustment outcomes, and more recent theoretical contributions have also been made with Berry's views on acculturative stress (1997) and by Ward and colleagues, who discuss crosscultural adaptation in relation to affective and behavioural acculturative changes (Ward, 2001a; Ward, Bochner, \& Furnham, 2001).

The current research programme, positioned within the field of acculturation psychology and set in an international context of cross-cultural transition, views crosscultural competence as an individual's potential capabilities (e.g., their knowledge, 
behavioural skills, and attitudes or motivation) that enable them to adapt effectively in crosscultural environments, and examines a behavioural component of cross-cultural competency known as sociocultural adaptation. As an adaptive measure of intercultural competency, sociocultural adaptation is situated within a theoretical framework of acculturation known as culture learning (e.g., Brislin, 1981; Furnham \& Bochner, 1986), and is defined here as an individual's ability to effectively acquire culturally appropriate skills and negotiate interactive aspects of a new cultural setting (Ward \& Kennedy, 1999). Sociocultural adaptation, as a behavioural outcome, has been differentiated between other adaptive outcomes such as psychological adjustment (Searle \& Ward, 1990; Ward, 1996; Ward \& Kennedy, 1996a), which involves affective or emotional responses experienced during crosscultural transitions. The following sections will provide further information about this sociocultural-psychological adjustment distinction, acculturation and culture learning paradigms, other behavioural outcomes of cultural competency, and the conceptualisation and measurement of the sociocultural adaptation construct.

\section{Acculturation and Adaptation: Theoretical Frameworks}

Acculturation is a process of cultural learning, where changes occur as a result of continuous first-hand contact between individuals of differing cultural backgrounds (Ward, 2001b). Empirical studies of acculturation processes began in the fields of sociology and anthropology, with historical accounts most often attributing the burgeoning of the topic to anthropologist Robert Redfield. In his much-cited "Memorandum for the Study of Acculturation" (Redfield, Linton, \& Herskovits, 1936), Redfield provided a general outline of the purpose of acculturation studies with the hopes of clarifying methodological and classification issues in the field. Redfield and colleagues also made strong distinctions between primitive and literate groups during this anthropological period of acculturation research at a societal level, in which an emphasis was placed on "acquiescence on the part of the members of the accepting [minority]" group (Redfield, Linton, \& Herskovits, 1936, p. 152). Acculturation research at this time was, in general, biased towards Western political and epistemological ideologies (e.g., Cheung, 1996; Sinha, 1987).

In the psychological field, Rudmin (2003) has recognised G. Stanley Hall as being the first psychologist to discuss acculturation. Hall's work was followed by social psychologists Znaniecki and Thomas and their research on Chicago immigrants, and later by British psychologist Frederic Bartlett, who may have been the first to highlight the importance of minority groups' attitudes towards majority groups. Psychologists became interested in 
acculturation psychology as an individual- rather than group-level phenomenon in the 1940's and 1950's, which helped formalise the term "psychological acculturation" (Sam, 2006, p. 14). One of the most prominent theorists in the acculturation literature is John Berry who, from the 1970's, has helped to shape the current state of acculturation psychology through his explication of acculturation strategies (integration, assimilation, separation, and marginalisation) regarding an individual's orientation towards their home and host culture (Berry, 1976, 2006, 2009).

Berry and colleagues have also contributed to the acculturation psychology field through further differentiating the concepts of acculturation, enculturation, and cultural change. Acculturation is a result of second-culture acquisition through intercultural contact, whereas enculturation is a process occurring during an individual's development of encoding cultural norms and values that, along with socialisation, allows for cultural transmission within a culture (Berry, Poortinga, Segall, \& Dasen, 1992; Castro, 2003). These two constructs contrast with cultural change, a construct concerning population-level changes that occur as a result of internal events such as intracultural discovery and innovation rather than contact with other cultures (Berry, 1995; Castro, 2003).

According to Sam (2006), there are three main steps involved in the acculturation process. One step in this process is continuous and first-hand contact between individuals or groups of disparate cultural backgrounds. Sam (2006) and Berry (1990) have suggested three broad dimensions of contact that differ between acculturating groups and individuals: Permanent-temporary, voluntary-involuntary, and sedentary-migrant. For instance, indigenous people have permanent and sedentary cultural contact with another cultural group, whereas refugees and sojourners have more temporary contact. In terms of the voluntaryinvoluntary continuum, asylum seekers have involuntary contact with another cultural group, whereas migrants or immigrants have more voluntary contact. The second step or "building block" of the acculturation process has been termed reciprocal influence, wherein two groups or individuals have influence on one another (Sam, 2006). Although power differences can contribute to one group-normally the dominant group-having more influence than the other, both dominant and non-dominant group change does occur. The third acculturative step involves change, which has been examined both in terms of the process of change as well as outcomes that take place as a result of change. In addition to psychological acculturation, the general topic of this research programme, other types of acculturation can include cultural, economic, political, biological, and physical change (Berry et al., 1992; Sam, 2006). 
As was previously mentioned, one of the most prominent theoretical frameworks used to conceptualise acculturation outcomes, the ABC model of culture contact (Ward, 2001b; Ward, Bochner, \& Furnham, 2001), categorises cross-cultural adaptation into three general groups: Affective, cognitive, and behavioural acculturative change (see Figure 1). Research conducted within the affective domain has a stress and coping focus centred on feelings of anxiety, well-being, or satisfaction during cross-cultural encounters, and the coping strategies an individual employs in these situations (Berry, 2006; Lazarus \& Folkman, 1984). Beginning with a foundation in psychiatry, the affective domain of acculturative change once held more of a psychopathological perspective that emphasised the negative consequences of intercultural contact (Ward, 1996). For example, the term culture shock, falling within this theoretical category, was described as an "occupational disease... precipitated by the anxiety that results from losing all our familiar signs and symbols of social interaction" in a foreign environment (Oberg, 1960, p. 177). More positive approaches within the stress and coping framework have since emerged, such as Berry's acculturative stress model (1970) and Lazarus and Folkman's work on stress and coping (1984). These later theories have posited that negative as well as positive life changes affect stress levels, which in turn prompt various coping strategies that can result in either adaptive or maladaptive outcomes (Ward, 1996). Further, as was briefly mentioned in the previous section, Ward and Kennedy (1999) proposed that emotive/affective outcomes falling within this domain be termed psychological adjustment as a way of distinguishing between other types of adaptive outcomes such as sociocultural adaptation. Additional information regarding the differentiation between these adaptive outcomes will be provided in the forthcoming section.

The cognitive domain of cross-cultural adaptation is based in social identification theories concerning how individuals consider and perceive themselves and others. For instance, social identity theory or SIT (Tajfel \& Turner, 1979; Tajfel, 1981) has emphasised the perceptions and categorisation of the self and others within the intercultural context (Ward, 2001b). At a group level, SIT considers issues pertaining to boundary permeability, power and status, and in- versus out-group differences and similarities (Turner, 1999; Ward, Bochner, \& Furnham, 2001). At an individual level, the potential discrimination or prejudice an individual who belongs to a minority group experiences from a majority group may cause a variety of reactions (Tajfel \& Turner, 1986), including increasing their in-group identification or leaving their group to find more positive opportunities for self-identification elsewhere (Brown, 2000). Phinney and colleagues (2001) suggested that these reactions depend on the characteristics and attitudes of immigrants, their group's specific 
circumstances, as well as the counteraction of the receiving society such as support or rejection of ethnic maintenance. Changes to in-group identity and an immigrant's sense of self may be exacerbated during the acculturative process, particularly with regard to disparate psychological outcomes (Berry, 1997).

Last, the behavioural or culture learning approach focusses on communication issues that arise within intercultural contexts, and highlights the importance of an individual's ability to acquire culturally appropriate skills and establish personal relationships that result in successful behavioural outcomes (Bochner, 1981; Furnham \& Bochner, 1986; Gudykunst \& Hammer, 1988; Hannigan, 1990). This theoretical domain of acculturative change will be further described in the following section.

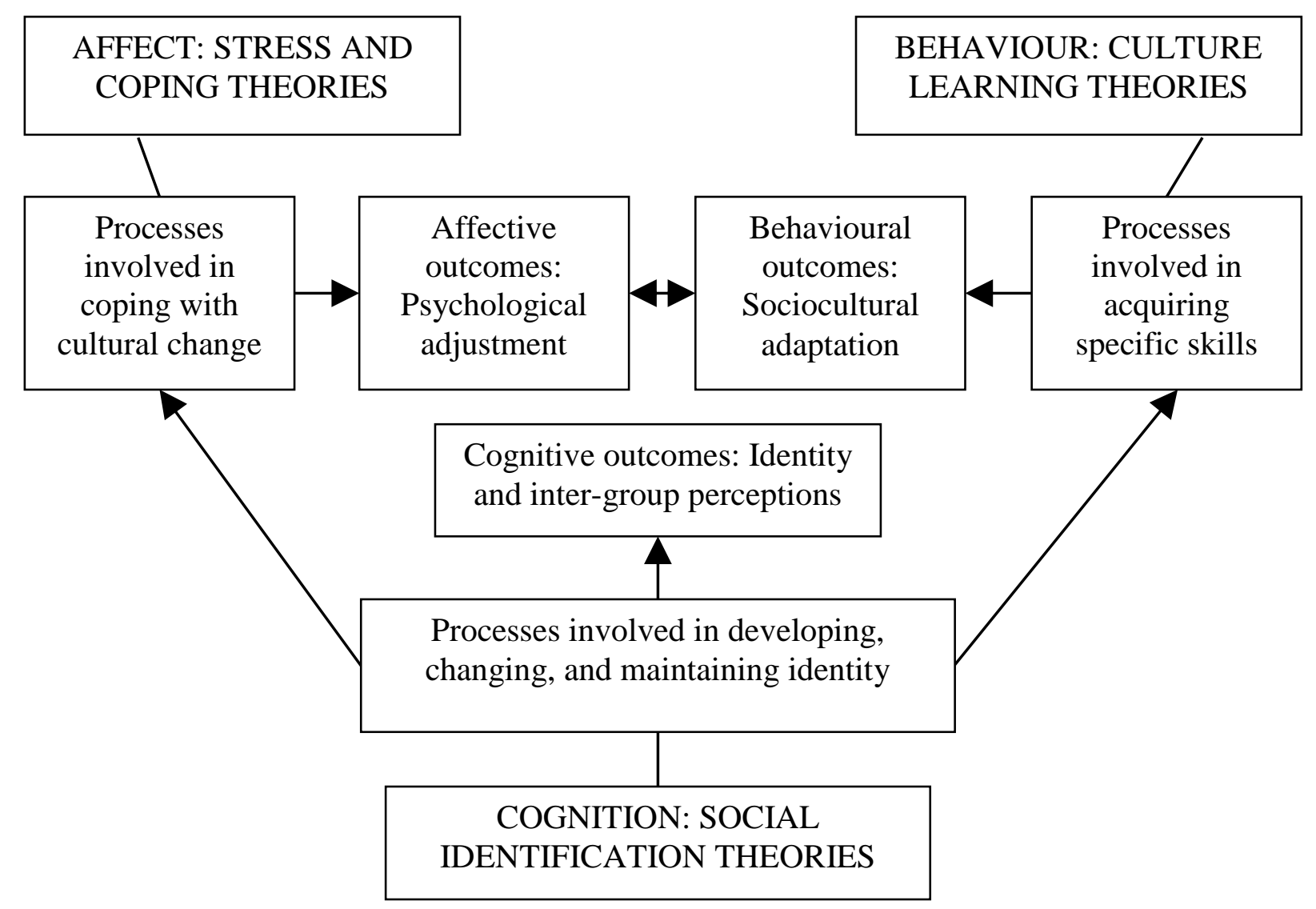

Figure 1. The ABC model of culture contact. Reproduced from "The A, B, Cs of Acculturation" by C. Ward, 2001, p. 416. In D. Matsumoto (Ed.), The Handbook of Culture and Psychology. Copyright 2001 by the Oxford University Press.

In sum, these three approaches to acculturative change-stress and coping, social identity theory, and culture learning-have disparate theoretical foundations derived from experimental, social, health, and developmental psychology. However, these domains are interrelated and can act as an overarching framework for the study of acculturation 
psychology (Ward, 2001b), specifically with regard to the sociocultural adaptation construct and its theoretical placement within the culture learning paradigm.

\section{Inside the Acculturative Framework: The Culture Learning Paradigm}

In the previous section, cross-cultural adaptation was described in terms of three overarching theoretical frameworks: Stress and coping, social identification, and culture learning. The following section provides an in-depth examination of the culture learning approach; an acculturative process that involves acquisition of culturally appropriate skills to assist in alleviating intercultural difficulties attributed to cross-cultural differences.

Culture learning was founded within an experimental research paradigm of social psychology that considered social behaviour as a mutual performance between individuals, and specifically as a performance that required regulation in order to avoid communication difficulties (Argyle, 1969; Argyle \& Kendon, 1967). Such interpersonal communication problems were thought to arise as a result of missed or absent social cues, including the verbal and non-verbal expression of emotions and feelings, gestures and postures, gaze patterns, and the performance of routines or social norms such as greetings and departures (Trower, Bryant, \& Argyle, 1979).

This interpersonal communication premise was later developed into the culture learning approach, where social interactions were considered in an intercultural rather than intracultural context (Bochner, 1972, 1981; Furnham \& Bochner, 1986). Researchers who contributed to the development of the culture learning framework highlighted the importance of identifying cross-cultural communication differences between cultures. For example, the culture learning paradigm assumed that, upon arrival to a new host culture, a sojourner would likely be unfamiliar with the social interaction patterns of the culture and therefore unaware of and socially unskilled in communication differences between his or her home and host culture environments. Accordingly, individuals who were able to recognise and understand the salient content of these intercultural communication differences were believed to more easily acquire, develop, and utilise cultural specific skills in order to successfully navigate intercultural situations (Furnham \& Bochner, 1982a; Taft, 1977).

As a result of this initial culture learning approach, a large breath of research has been conducted on identifying the verbal and non-verbal communication differences that exist between cultures with the belief that transitioning individuals who recognised and managed these cross-cultural differences would be more successful at adapting to a new cultural context. Language proficiency in a host society has been considered to be a central 
component of the culture learning framework, as language is "the primary medium through which cultural information is communicated", and the absence of verbal skills in a new cultural environment will likely result in communication misunderstandings (Masgoret \& Ward, 2006, p. 63). In particular, many researchers have asserted that language proficiency, linguistic self-confidence, and communication competence are essential for the completion of day-to-day tasks in a foreign environment, building meaningful interpersonal relationships and social support networks with members of the host society, and successful acculturation in general (Church, 1982; Clément \& Bourhis, 1996; Kang, 2006; Masgoret, 2006; Masgoret \& Ward, 2006; Noels, Pon, \& Clément, 1996; Ward, 2004; Ward \& Kennedy, 1993a).

In addition to language competency, other forms of communication have also been found to vary across cultures. For instance, high versus low context communication has been studied (Hall, 1976; Gudykunst \& Matsumoto, 1996; Ting-Toomey, 1988) in terms of how directly or indirectly information is provided within a cultural context. Messages or communication in low context countries (e.g., generally individualistic nations such as the United States) have been shown to be more explicit or unambiguous in nature, whereas high context countries such as Japan that are more collectivistic appear to rely less on explicit messages and more on an individual's internalisation of the message and the physical context in which the message is transmitted (Gudykunst \& Matsumoto, 1996; Hall, 1976). This cultural variability can result in individuals from high-context cultures expecting more out of other people than individuals who were raised in low-context cultures:

When talking about something that they have on their minds, a high-context individual will expect his [or her] interlocutor to know what's bothering him [or her], so that he [or she] doesn't have to be specific. The result is that he [or she] will talk around and around the point... putting all the pieces in place except the crucial one. Placing it properly... is the role of his [or her] interlocutor. (Hall, 1976, p. 98)

Other research on disparities in communicating across cultures involves gestures, an important component of non-verbal communication that enhance verbal messages and shared understanding (Efron, 1941; Kendon, 1997; Levenson, Ekman, \& Friesen, 1990; Molinsky, 2007; Molinsky, Krabbenhoft, Ambady, \& Choi, 2005). Past research has indicated that nonverbal gestures differ significantly between cultures (Archer, 1997; Kendon, 1992; Poortinga, Schoots, \& Van de Koppel, 1993; Safadi \& Valentine, 1998). For example, the "okay" symbol in the United States (forefinger and thumb form a circle with other fingers held 
upright) is the symbol for "money" in Japan, and the gesture indicating "anger" in Nepal is the same as that signifying "fear" in Mexico (Archer, 1997). Whereas individuals born and raised in their home country develop an implicit, automatic understanding of non-verbal gestures through socialisation (Archer, 1997; Collett, 1993), cross-cultural sojourners must become competent with the non-verbal language of a new culture through the explicit acquisition of gestural recognition and by learning the meanings associated with these gestures (Elfenbein \& Ambady, 2003; Molinsky, 2007).

Emotive facial expressions, another aspect of non-verbal communication, are an imperative component of successful intercultural communication that varies interculturally. Emotive facial expressions across cultures include cultural display rules (Matsumoto, 1990), the inter-generational transmission of emotional expressions or displays concerning the appropriateness of behavioural responses in various situations and contexts, as well as cultural decoding rules (Buck, 1984; Matsumoto \& Ekman, 1989); the culture-specific perception of emotive facial expressions and how they are understood or interpreted. Several cross-cultural comparative studies conducted by Matsumoto and colleagues have shown cultural differences in emotion recognition and displays of emotive intensity (Ekman, 1992; Matsumoto, 1989, 1990; Matsumoto \& Ekman, 1989; Matsumoto, Kasri, \& Kooken, 1999), and further work has indicated that exposure to and recognition of culture-specific facial expressions influences successful interpersonal communication skills, the establishment of relationships, and overall intercultural competence (Beaupré \& Hess, 2006; Elfenbein \& Ambady, 2003; Kang, Shaver, Sue, Min, \& Jing, 2003; Porter \& Samovar, 1998; Yoo, Matsumoto, \& LeRoux, 2006).

There are several other components of non-verbal communication differences known to affect an individual's adaptation to a new cultural context. Physical contact and personal space, for instance, are types of non-verbal communication that can be categorised into high versus low-contact (Hayduk, 1983; Ward, 2001a). In high contact cultures (i.e., Latin America and Mediterranean countries), frequently-occurring contact like embraces or armtouching, and close standing and sitting proximal distance in conversations create more sensory input and a sense of immediacy that is generally a cultural norm (Hall \& Hall, 1990; Sussman \& Rosenfield, 1982). This contrasts with low-contact countries (i.e., northern European countries, various Asian countries) in which less bodily contact and greater personal space between communicators are more commonplace (McDaniel \& Anderson, 1998). Interestingly, low-contact countries are generally further away from the equator and high-contact societies generally closer to the equator, which has led some researchers to 
hypothesise that low-contact societies in cooler climates must be more organised, constrained, and structured to manage harsher environmental conditions than those in warmer regions (Anderson, Lustig, \& Anderson, 1990; Pennebaker, Rimé, \& Blakenship, 1996).

Eye contact or mutual gaze frequency also differs across cultures. Mutual gaze frequency has several functions in social interactions, such as the regulation of the beginning, ending, or maintaining of a conversation; assessment of others' behaviour and character judgments; and as an aid in the expression of an emotive state (Kendon, 1967; LaFrance \& Mayo, 1976; McCarthy, Lee, Itakura, \& Muir, 2006; Rule, Ambady, Adams, \& Macrae, 2008). Cross-cultural differences in these functions of gaze or eye contact include both duration and direction of eye gaze (Knapp \& Hall, 2002). Indigenous North American Navaho Indians and Japanese have been found to judge steady eye contact negatively, for example, whereas North American Europeans and Trinidadians positively judged eye contact (Bull \& Gibson-Robinson, 1981; Hornik, 1987; McCarthy et al., 2006). Further, North American Europeans may perceive looking up to represent thinking and looking down to represent deceitfulness or insecurity, which differs from Navaho Indians, Ethiopians, and Japanese, who generally consider looking down as a symbol of respect (Argyle \& Cook, 1976; Collett, 1971). Additionally, cultural differences in an individual's ability to infer others' mental states from the eyes alone have been evidenced in an fMRI investigation where Japanese and Americans of European descent performed better on same versus otherculture mental state decoding from the eyes (Adams et al., 2009).

There are also differences in the way individuals from different cultures use silence in communication. Silence provides a background or foundation for meaning as a conversation unfolds, and language can be considered all the more consequential when contrasted against these related silences (Johannesen, 1974). Culture has an effect on how individuals use and perceive silence, as well as meanings they attach to it (Hasegawa \& Gudykunst, 1998; Enniger, 1987; Jaworski, 1993). A considerable amount of research has been done comparing the different definitions and uses of silence in Japanese and Americans. Japanese, for instance, consider the word for silence to mean keeping quiet or not speaking, and Americans define silence as an absence of sound or lack of verbal communication (Giles, Coupland, \& Wiemann, 1992). Additionally, as was previously described, low-context communication is more emphasised in individualistic societies. Use of silence in these countries occurs more infrequently as silence does not contain meaning in the communicated message, which suggests that Americans may be more consciously aware of their use of silence (Hasegawa \& Gudykunst, 1998). High-context cultures such as Japan are more often 
collectivistic in nature, and silence is thus more often employed and Japanese people are generally not aware of their use of silence (Miyahira, 1991). The use of silence by Japanese may be a sign of politeness, respect, or a desire to continue speaking after making a point rather than a lack of interest in continuing to speak (Sue \& Sue, 1977).

Conventions or norms that regulate interpersonal communication may also be a source of polarity in intercultural situations. Normative rules of communication behaviours can be attributed to variations in the cultural dimensions of values-defined as a set of abstract, desirable goals that assist in the pursuit of life goals (Rokeach, 1973; Smith, Bond, \& Kagitçibaşi, 2006) — at both the national and individual level. Prominent research programmes have examined variations and similarities in values across cultures (Hofstede, 1980, 1983, 2001; Schwartz, 1992, 1994), and, in part, appear to reflect the different ways individuals from various cultures communicate in terms of volume of speech, directness of approach, how turn-taking is distributed, and forms of address indicating status (Masgoret \& Ward, 2006). However, some research has found that adopting new cultural values in order to achieve more successful intercultural communication and adjustment may not be as crucial as obtaining a deeper understanding and awareness of differences in cultural values (Kurman \& Ronen-Eilon, 2004; Ward \& Searle, 1991).

Other cultural dimensions and theories used to study variability in intercultural communication also exist. For example, face negotiation theory (Ting-Toomey, 1988; TingToomey \& Kurogi, 1998) examines how cultures differ in their management strategies to save, honour, or maintain face; the projected image of one's self, particularly in conflict situations. Expectancy violation (Burgoon, 1978, 1995) is another communication theory related to how interpersonal contact varies as a function of the expectations an individual holds for behavioural conduct and responses to violations of these expectations. Other theories include conversational constraints theory (Kim, 1995), which concerns how messages are constructed in terms of social-relational or task-oriented constraints; and communication accommodation theory, a theory that examines how individuals exhibit their opinions about and attitudes towards another vis-à-vis verbal or non-verbal convergence, divergence, or maintenance (Gallois, Giles, Jones, Cargile, \& Ota, 1995).

In summary, cross-cultural communication is a central aspect of the culture learning paradigm. Specifically, initial approaches in the area emphasised the identification of cultural differences in communication styles, as it was believed that a better understanding of behavioural norms would enable individuals to more quickly and successfully learn culturespecific cognitive and performance skills required in new situations. Ultimately, the culture 
learning approach stressed the importance of understanding intercultural communication differences and the ability to acquire new culture-specific communication skills in order for an individual to be effective and successful in a new culture.

\section{Advancement of the Culture Learning Paradigm}

The previous section described how research within the culture learning framework first focussed on the identification and comparison of cross-cultural communication differences in order for individuals to learn culture-specific skills necessary for successful intercultural adjustment. Several advances within the culture learning framework since its initial development have been made. As Masgoret and Ward (2006) have noted, for example, culture learning theory has been combined with other theoretical approaches concerning the psychological processes behind social encounters. These approaches include communication styles and intercultural communication competence theories developed by researchers such as Gudykunst (Gudykunst \& Kim, 1984), Kim (1991, 1995), and others (Gallois, FranklynStokes, Giles \& Coupland, 1988). Other theoretical approaches to culture learning theory include Bhawuk and colleagues' "triple-loop" learning process (Bhawuk, Sakuda, \& Munusamy, 2008), incorporation of the framework into social learning and cultural evolutionary theories, and application of the culture learning approach to cross-cultural training programmes.

One theoretical development within the culture learning paradigm is the "triple-loop" learning process delineated by Bhawuk and colleagues (Bhawuk, Sakuda, \& Munusamy, 2008) in relation to their theory of intercultural sensitivity. They have argued that development of an individual's intercultural sensitivity and cultural intelligence depends on the learning that takes place within intercultural interactions, and outlined a three-step process involving information gathering, information comparison, and enactment of behavioural strategies within cross-cultural interactions.

A potential theoretical advancement for culture learning research not yet widely considered in the literature relates to a corresponding social psychology paradigm known as social learning, and in turn to prospective associations between social learning and contemporary cultural evolutionary theories. Social learning theory is a learning theory that provides a basis for understanding behaviour across (intercultural) and within (intracultural) cultures in terms of intercultural adjustment, cross-cultural and management training, motivational aspects of self-efficacy, and organisational behaviour (Bandura, 2006; Black \& Mendenhall, 1990; Davis \& Luthans, 1980; Latham, Fay, \& Saari, 1979; Noe, 1986). 
Specifically, Bandura (1977) suggested that human behaviour is acquired more predominantly via imitation of others' behaviour (e.g., observational or social learning) than through individual learning (i.e., trial and error) or genetic heredity.

In terms of the relational overlap between culture learning and social learning, culture learning theory concerns cultural transition and skill acquisition, but the actual process by which an individual acquires these skills in intracultural or intercultural contexts is through social learning. Using this existing overlap in culture and social learning theories as a foundation, work done in the cultural evolutionary literature may be a new and interesting avenue of research inquiry to apply to the culture learning and intracultural communication fields.

In the broadest sense, cultural evolutionary theory considers culture as socially transmitted information, and many researchers consider this culturally acquired information to be transmitted genetically or biologically, much like Darwinian evolutionary processes (Richerson \& Boyd, 2005; Mesoudi, 2009). Cultural evolutionary theory and social psychology can mutually inform one another through focussing on the process of selective learning a person experiences during cultural change. For example, some work (Mesoudi, 2008; Rogers, 1988) has shown that when an environment changes too rapidly, people who learn individually do better in detecting these environmental shifts in comparison to social learners, who may be left imitating irrelevant or obsolete behaviours. With this in mind, work from the social learning and cultural evolutionary theories could be used to examine to what extent cultural change affects the selective processes by which an individual acquires information, as well as the interplay of individual versus social learning.

In addition to the aforementioned theoretical advances, the culture learning approach has also formed the foundation for many applied intercultural training programmes. Curriculums have, for example, incorporated Trower, Bryant, and Argyle's (1979) emphasis on specific learning elements such as guidance, practice, and feedback in the development of intercultural effectiveness using behavioural training methodologies (Gudykunst, Hammer, \& Wiseman, 1977). Others have taken elements from culture learning to examine the interpersonal and intercultural components known to facilitate social skill acquisition, and some have utilised the culture learning paradigm to analyse cross-cultural similarities and differences in the prediction of successful adaptation when implementing culture-general and culture-specific training methods (Ward, 1996). The significance that the culture learning framework places on culture-specific skills acquisition also parallels cross-cultural training literature authored by Black, Mendenhall, and Oddou (Black \& Mendenhall, 1990; 
Mendenhall \& Oddou, 1985, as cited in Blake, Heslin, \& Curtis, 1996). Black and colleagues have considered the cross-cultural training process as a learning exercise which requires an individual to adopt new skill sets within three main areas of cultural transition: The self, where skills are developed to improve and maintain self-confidence and well-being; the other, whereby behavioural skills are espoused to foster host national relationships; and perception, where the acquisition of new skills promotes appropriate attitudes and beliefs towards the host culture.

The current section outlined more recent theoretical influences of the culture learning framework on communication across and within cultures, introduced a novel theoretical approach to the culture learning paradigm that incorporates cultural evolutionary psychology and social learning theory, and examined the applied influence of culture learning on crosscultural training programmes. With regard to the current research programme, consideration of the culture learning paradigm will be approached in terms of how the framework has evolved to incorporate the definition and prediction of sociocultural adaptation.

\section{Adaptation within the Behavioural Domain of Cultural Competency}

As was mentioned previously, adaptive outcomes of cultural transition have been viewed across a variety of cognitive, intrapersonal, and behavioural skill domains such as relational, identity, health, task-specific, and psychological indices. The present research programme takes a behavioural skills approach to the investigation of adaptive outcomes within the culture learning framework through an emphasis on Ward and colleagues' investigation of the sociocultural adaptation construct and its distinction from psychological indices of cross-cultural adjustment (Ward, 1996; Ward \& Kennedy, 1999). The differences between these two adaptive outcomes will be discussed at the conclusion of this section; first, other corresponding conceptualisations and adaptive outcomes indices within the behavioural skills domain of cultural competency will be examined including research conducted by Hammer, Gudykunst, and Wiseman (1978); Furham and Bochner (1982b); Black and Stephens (Black, 1988; Black \& Stephens, 1989); and Mak and associates (Mak, Westwood, Barker, \& Ishiyama, 1998).

One conceptualisation of behavioural intercultural effectiveness was described in Hammer, Gudykunst, and Wiseman's factor analytic study (1978) of Americans' effective functioning in a foreign culture. These sojourners self-reported how much they believed that 24 different personality abilities such as perceptive skills, interaction management, and anxiety management skills related to their intercultural competency. These self-reports were 
factor analysed and found to have three general behavioural dimensions: (a) The ability to initiate interpersonal relationships; (b) the ability to communicate effectively; and (c) the ability to manage intercultural stress (Hammer, Gudykunst, and Wiseman, 1978). Hammer (1987) later replicated these findings to offer additional confirmation of the importance of these three general abilities in facilitating sojourner effectiveness in a new cultural environment.

Gudykunst and Hammer (1988) went on to generate a communication-based theory of intercultural adaptation based on the theory of uncertainty reduction (Berger, 1979). In particular, Gudykunst and colleagues speculated that the difficulties experienced with intercultural adaptation can be assuaged by a sojourner becoming more confident and less anxious about how to behave in their host society (Gao \& Gudykunst, 1990), and found that variables such as knowledge of the host culture, favourable contact with host nationals, cultural similarity (e.g., less cultural distance), and competence in the host language mediated this uncertainty and anxiety (Gudykunst \& Hammer, 1988). Gao and Gudykunst (1990) found support for this theoretical approach, and also suggested implications of the theory for various intercultural training exercises and simulations.

Furnham and Bochner (1982b) developed another conceptualisation and measurement of behavioural adaptation. In their empirical analysis of international students' abilities to negotiate social encounters in new cultural environments, these researchers created the Social Situations Questionnaire, and with use of the instrument concluded that international students living in Britain found greatest difficulty in establishing and maintaining personal relationships with host nationals. They also determined that the greater degree of disparity or cultural distance between Britain and the students' home culture, the greater amount of difficulty the students experienced in negotiating social interactions.

Black and colleagues were some of the first researchers to examine differing dimensions of behavioural skills adjustment (Black, 1988; Black \& Stephens, 1989). Based on previous work by Torbiorn (1982), Black (1988) developed a measurement of adjustment consisting of three behavioural facets: Adjustment to a professional work role in the host country; adjustment to host national interactions; and general adjustment to factors outside of the work environment. In using this scale to investigate American expatriate adjustment to Japanese culture, Black's multi-dimensional adjustment construct challenged extant theoretical suppositions that behavioural adaptation was a "generic or unitary phenomenon" (1988, p. 289). This three-dimensional model of adjustment has since been utilised in a large breadth of international business, expatriate, and organisational management literature (e.g., 
Ali, Van der Zee, \& Sanders, 2003; Parker \& McEvoy, 1993; Shaffer, Harrison, \& Gilley, 1999).

Of further relevance to the behavioural skills approach is the ExcelL programme developed by Anita Mak and colleagues (Mak, Westwood, Barker, \& Ishiyama, 1998). The ExcelL programme, based on Bandura's (1986) social cognitive learning paradigm, is an experiential- and knowledge-based course that assists newcomers in developing successful social interactions skills with host nationals (Mak \& Barker, 2004) through behavioural competence training in areas such as making social contact, seeking help, participation in a group, expressing disagreement, and giving feedback (Mak \& Buckingham, 2007). The programme aims to improve both an individual's intercultural competence and social selfefficacy through social skills training in order to increase sociocultural adjustment (2007).

The current research, situated within the culture learning framework, also takes a behavioural skills approach to the investigation of adaptive outcomes through its emphasis on the sociocultural adaptation construct, and the distinction Ward and colleagues have made between sociocultural adaptation and psychological indices of cross-cultural adjustment (Searle \& Ward, 1990; Stone Feinstein \& Ward, 1990; Ward, 1996; Ward \& Kennedy,1992a, 1993a; Ward \& Searle, 1991). Whereas psychological adjustment involves affective or emotive outcomes of cross-cultural transition such as depression or well-being (Ward, 2001a; Ward \& Kennedy, 1999), sociocultural adaptation describes behavioural outcomes of intercultural transition that relate to an individual's ability to learn culturally appropriate skills and negotiate interactive aspects of a new cultural setting (Ward \& Kennedy, 1999). Ward and colleagues have also examined how the two domains differ in terms of patterns of change over time (Ward \& Kennedy, 1996a; Ward, Okura, Kennedy, \& Kojima, 1998), and how predictive models of psychological adjustment and sociocultural adaptation contrast with one another (Searle \& Ward, 1990; Ward \& Kennedy, 1992b; Ward \& Searle, 1991).

One empirical distinction between psychological and sociocultural adjustment relates to their respective patterns of adjustment over time. For example, sociocultural adaptation has been found to improve consistently over time in a general approximation of a learning curve, whereas psychological adjustment has shown greater instability and variation (Ward \& Kennedy, 1996a). Further, in a longitudinal study of the relationship between the two adjustment domains involving Japanese students in New Zealand (Ward, Okura, Kennedy, \& Kojima, 1998), data analyses from four separate time periods (24 hours to one year) revealed significant differences in the psychological-sociocultural adjustment relationship between 
initial entry into New Zealand and one year, indicating that the magnitude of this relationship increases with time, at least as was the case for this particular sojourning group.

Research has also found psychological adjustment and sociocultural adaptation to be affected by different variables (Sam, Vedder, Ward, \& Horenczyk, 2006). Psychological adjustment has been associated with life changes (Searle \& Ward, 1990), social support (Adelman, 1988; Oppedal, Røysamb, \& Sam, 2004; Ward \& Chang, 1997), various personality dimensions (Bakker, Van Oudenhoven, \& Van der Zee, 2004; Van Oudenhoven \& Van der Zee, 2002; Ward \& Fischer, 2008), and coping strategies (Berno \& Ward, 1998; Stone Feinstein \& Ward, 1990; Ward \& Kennedy, 1999; Ward \& Rana-Deuba, 1999). Sociocultural adaptation, contrastingly, has typically been shown to be predicted by factors such as cultural knowledge (Kurman \& Ronen-Eilon, 2004; Li \& Gasser, 2005; Ward \& Kennedy, 1999), cultural distance (Searle \& Ward, 1990; Zlobina, Basabe, Paez, \& Furnham, 2006), identification and interaction with host nationals (Ataca \& Berry, 2002; Li \& Gasser, 2005; Ward \& Rana-Deuba, 1999), length of residence in a new host society (Ward \& Kennedy, 1996a; Ward, Okura, Kennedy, \& Kojima, 1998), and language fluency (Masgoret, 2006; Ward \& Kennedy, 1994, 1999).

Further, differences in sojourners' opportunities, needs, and capacity for integration into the host culture also creates disparities in adaptive outcomes and the relationship between the adjustive outcomes themselves (Ward \& Kennedy, 1996). For instance, minimal interaction with host nationals has been found to weaken the strength of the relationship between psychological adjustment and sociocultural adaptation, whereas integrated sojourners report greater associations between these adjustment domains.

Despite the differences between psychological and sociocultural adaptation, both adaptive outcomes have been found to be affected by certain contextual factors. For example, Berry (1997) has described a number of factors present in an immigrant's country of settlement that contribute to acculturation outcomes, such as the host society's policies towards immigration and pluralism, and the relative acceptance of specific religious and ethnic groups. Further, both psychological and sociocultural acculturation outcomes appear to be influenced by host nationals' perceptions of and attitudes towards various immigrant groups, as burgeoning research in this area suggests (Kim, 1999; Lalonde \& Cameron, 1993; Liebkind, 2001; Piontkowski, Florack, Hoilker, \& Obdrzalek, 2000).

The Interactive Acculturation Model (IAM) formulated by Bourhis and colleagues (Bourhis, Moïse, Perreault, \& Senecal, 1997; Montreuil \& Bourhis, 2001) is one perspective on majority-minority acculturation dynamics that suggests the attitudes of host majority 
group members towards acculturation orientations can change those orientations held by immigrant group members, "yielding relational outcomes [relative 'fit'] that may be harmonious, problematic, or conflictual" (2001, p. 700). A growing body of research has investigated the association between the relative fit of majority-minority groups in terms of concordant/discordant acculturation preferences and sociocultural and psychological adjustment including life satisfaction, successful intercultural adjustment, and competence (Horenczyk, 1996; Kurman, Eshel, \& Sbeit, 2005; Jasinskaja-Lahti, Liebkind, Horenczyk, \& Schmitz, 2003; Kus \& Ward, 2009; Schwartz, Roccas, \& Horenczyk, 2000; Van Selm, Sam, $\&$ Van Oudenhoven, 1997). These studies strengthen the premise that societal contexts, including the extent to which majority group members accept minority groups, immigrants' circumstances, and the perceptions immigrants hold of majority members' attitudes towards them, have a substantial influence on both sociocultural and psychological adjustment outcomes (Chirkov, 2009a; Ward, Fox, Wilson, Stuart, \& Kus, 2010; Ward, Okura, Kennedy, \& Kojima, 1998).

\section{Measurement of Sociocultural Adaptation}

One of the most prominent challenges in the field of acculturation psychology has involved the conceptualisation and measurement of cross-cultural adjustment. A multitude of researchers have voiced their concern over the ambiguous and undifferentiated use of terms such as culture shock, adjustment, adaptation, acculturation, assimilation, cross-cultural competence, and cultural effectiveness, and how a lack of consensus about the operational definitions of these terms has impeded more rigorous empirical inquiry (see Abbe, Gulick, \& Herman, 2008; Benson, 1978; Collier, 1989; Hannigan, 1990; Johnson, Lenartowicz, \& Apud, 2006; Kealey, 1989; Ruben, 1989; Rudmin, 2003, 2009; Rudmin \& Ahmadzadeh, 2001). To address these concerns, the following section examines the operationalisation of the sociocultural adjustment construct.

The measurement of sociocultural adaptation as an adaptive, behaviourally-based outcome of cultural competence can first be traced back to the work of Argyle and colleagues on social competence, where self-reported degree of difficulty was measured as an index of social skill across situational categories such as situations requiring assertiveness, formal social occasions, and meeting strangers (Argyle, 1969; Argyle, Furnham, \& Graham, 1981; Trower, Bryant, \& Argyle, 1978). Searle and Ward (1990) modified this measure, known as the Social Situation Competence Scale (Bryant \& Trower, 1974), to create the first version of the Sociocultural Adaptation Scale or SCAS. The SCAS was conceptualised as the 
acquisition of behavioural skills required for an individual to negotiate life in new cultural environments, and was measured in terms of the degree of self-reported difficulty experienced in interpersonal situations and with the accomplishment of day-to-day tasks.

Since its inception, the SCAS has been utilised both in a programme of acculturation research and across other disciplines including communication and language acquisition, education, international business and management, and organisational psychology (Kim, 2009; Lai, 2006; Townsend \& Wan, 2007; Yu, 2010; Zhang, 2005). Psychometric development of the SCAS spanned approximately 9 years using data from 16 cross-sectional and four longitudinal samples (Ward \& Kennedy, 1999), and based on the empirical support provided by these studies, Gudykunst (1999, p. 553) evaluated the instrument as having "the most empirical foundation of any measure used in the study of intercultural relations" and as "a highly reliable, valid, and versatile measure of behavioural adaptability." In sum, the SCAS is one of the first in the acculturation field to address the discrepant and often varied ways in which sojourner adjustment had previously been defined, measured, and interpreted through its use of the more systematic and theoretically-driven acculturation framework.

\section{The Current Research Programme}

The current research programme provides an in-depth examination of sociocultural adaptation and the behavioural competencies individuals acquire when living in a new cultural environment. It considers factors that both assist and hinder this acculturative process, and how culturally-based behavioural competencies are defined and measured within the acculturation psychology domain. Ultimately, the following work aims to provide a more concise conceptual and operational understanding of immigrants' adaptation and settlement within a new country, including the contextual and interpersonal variables that contribute to their adjustment.

The following studies included in this thesis address three central issues concerning the review, revision, and expansion of work on the topic of sociocultural adaptation. Chapter 2 consists of the first empirical investigation (Study 1), which provides a quantitative review in the form of a meta-analysis regarding the correlates or antecedents of sociocultural adaptation as measured by the SCAS. Along with providing an in-depth review of the theoretical origins of sociocultural adaptation, a central question is also examined: What aspects of a migrating individual's personal history (e.g., situational factors such as previous cross-cultural experience and foreign language ability) and personality contribute to their successful adjustment to living and working in a foreign environment? What are the 
theoretical foundations of these assumed associations with adjustment difficulty, and what do the meta-analytic findings suggest in terms of future research concerning these antecedents?

In Chapter 3 (Study 2), a second issue is addressed concerning how researchers and practitioners working in the field of diversity and settlement approach the measurement or quantification of sojourner adjustment. How valid is the current methodology used within the acculturation field to measure the sociocultural adaptation construct? How well does this method relate to the construct's operational definition? To address these questions, Study 2 seeks to refine the measurement of sociocultural adaptation as a behavioural facet of cultural competency. Using exploratory factor analysis, existing issues with the construct as measured by the SCAS are addressed and resolved through development of a revised measure. This revised measure of sociocultural adaptation also explores behavioural competency in terms of specific ecological, interpersonal, language, and professional/work domains.

The fourth chapter of this research, Study 3, is based on findings from the first two studies in the research programme. First, this final study employs confirmatory factor analysis to replicate and validate findings from Study 2 with regard to the psychometric properties of the revised sociocultural adaptation measure. Second, path analysis techniques facilitate the examination of two underrepresented variables in the acculturation literature, motivation and perceived discrimination, which were found in Study 1 to have strong associations with successful cross-cultural adaptation. A novel approach to sociocultural adaptation is presented with two hypothetical path models integrating the two aforementioned constructs. In particular, these path models test direct and indirect pathways between reasons or factors for moving abroad, migration motivation (conceptualised by two different facets of cross-cultural motivation; autonomous regulation and Motivational CQ), sociocultural adaptation competency, perceived discrimination, and psychological well-being.

The fifth and final chapter of this thesis provides a summary of the main empirical findings from Studies 1, 2, and 3. The contributions, implications, and limitations of these studies are discussed in relation to the existing acculturation psychology literature. Directions for future research and potential application of the findings are also suggested. 


\section{Chapter Two: A Meta-Analysis of the}

\section{Correlates of Sociocultural Adaptation}

The previous chapter introduced the topic of cultural competence as positioned within the culture learning framework. Emphasis was placed on the concept of cultural competence and particularly sociocultural adaptation with regard to its conceptualisation, measurement, and differences that exist between this behavioural dimension of cross-cultural transition and other adaptive domains such as psychological adjustment. Specifically, differences between sociocultural adaptation and psychological adjustment were examined in terms of their theoretical backgrounds, how the two domains differ with regard to their patterns of change over time, and what disparate variables are thought to influence these two outcomes.

Commencement of this research programme on sociocultural adaptation begins with the present chapter, which details a meta-analytic review of the correlates of cultural competency as measured by the Sociocultural Adaptation Scale (SCAS; Ward \& Kennedy, 1999). The review seeks to address two primary issues. First, because theoretical and applied research on sociocultural adaptation has traditionally been situated within the culture learning framework, far less research has focussed on the influence of an individual's personality traits on their adaptation to a new sociocultural milieu, although some researchers have recognised the paucity of personality research in this area or have made appeals for this lack of research to be addressed (Berry, Poortinga, Breugelmans, Chasiotis, \& Sam, 2011; Kosic, 2006; Padilla \& Perez, 2003; Ward, 1996, 2001b). Second, no quantitative, systematic review based on empirical evidence using the SCAS has investigated how demographic, situational variables, other aspects of adaptation such as psychological adjustment, and individual differences including factors such as personality and motivation relate to an individual's adaptation to a new culture.

In order to address these issues, data from various studies utilising the SCAS were collected and analysed to examine a total of 21 variables. These variables included demographic factors (age and gender; $N=2$ ), as well as situational variables such as contact with host and co-nationals, cultural distance, cultural knowledge, previous cross-cultural experience, language ability, length of residence, and perceived discrimination $(N=8)$. Other factors included psychological adjustment $(N=1)$, and personality and motivation components such as the Big Five personality traits, cultural empathy, general and crosscultural self-efficacy, integrative motivation, and an amalgamated motivation construct $(N=$ 10). Results of the meta-analysis emphasise the importance of individual differences such as 
personality and motivation in relation to adaptation difficulties, and suggestions are provided for future theoretical and applied research regarding how demographic, situational, and individual differences components relate to and influence an individuals' cross-cultural adjustment.

\section{Review of Sociocultural Adaptation and the SCAS}

As was previously mentioned in Chapter 1, sociocultural adaptation is based in the culture learning paradigm, which highlights the understanding and acquisition of new behavioural repertoires within social interactions and communication to successfully navigate cross-cultural situations (Bochner, 1972, 1981; Furnham \& Bochner, 1982a, 1986; Taft, 1977). Sociocultural adaptation also relates to social learning theory, which suggests that human behaviour is acquired predominantly via imitation of others' behaviours rather than through individual learning. Both of these learning theories concern skill acquisition within the cultural transition process.

As a behavioural index of sociocultural adaptation, the SCAS is based on Argyle and colleagues' work concerning social competence (Argyle, 1969; Argyle, Furnham, \& Graham, 1981; Trower, Bryant, \& Argyle, 1978). Searle and Ward (1990) developed the SCAS based on this social competence foundation, and conceptualised sociocultural adaptation as the acquisition of behavioural skills needed for living in novel cultural environments as measured by difficulty associated with interpersonal and daily life situations. Development of the SCAS included over 20 cross-sectional and longitudinal samples (Ward \& Kennedy, 1999), and the scale has been considered a reliable and valid measure of behavioural adaptability with a strong empirical foundation (Gudykunst, 1999). By 2012, more than 100 studies had been published using the SCAS, and it is this research that establishes the basis of the current meta-analysis.

\section{Culture Learning Theory: Situational Factors and Sociocultural Adaptation}

As sociocultural adaptation is positioned within a culture learning framework and involves behavioural skills, research concerning its predictors or antecedents has largely focussed on situational variables associated with the learning process. Eight of these commonly investigated situational variables are included in the meta-analysis: Length of residence abroad, previous cross-cultural training or international experience, cultural knowledge, cultural distance, language fluency, host and co-national contact, and perceived discrimination. 
In a new cultural environment, knowledge and skill acquisition are necessary requirements for successful sociocultural adaptation. Some researchers have suggested that intercultural experience and cross-cultural training assist with the early stages of skill acquisition or culture learning, helping an individual move from layperson to cultural novice (Bhawuk \& Triandis, 1996), and much research has purported the positive effects of training on positive learning outcomes such as multicultural competency and life satisfaction (Arthur \& Achenbach, 2002; Fielder, Mitchell, \& Triandis, 1971; Fowler, 1994; Ponterotto, Fuertes, \& Chen, 2000; Pope-Davis, Breaux, \& Liu, 1997). Some debate exists regarding the generalisability of learning (e.g., the transfer of skill-based knowledge or experience from one context to another) to other settings (Bhawuk, 1998; Masgoret \& Ward, 2006), but a substantial amount of learning theory and research has indicated that learning generalisation does occur in cross-cultural contexts. Specifically, previous international experience has been associated with an increased ability to cope with everyday aspects of a new culture such as housing and shopping, work adjustment and better adjustment in general (Black, 1988; Klineberg \& Hull, 1979; Parker \& McEvoy, 1993; Pruitt, 1978). Based on these findings, a positive relationship between previous cross-cultural training or international experience and sociocultural adaptation is hypothesised.

Length of residence or time abroad in a host country can also be considered as a situational variable associated with the learning process. Temporal stages of learning have been applied to the topic of intercultural adjustment (e.g., psycho-emotional adjustment) in terms of the U-curve theory or UCT (Lysgaard, 1955). This theory involves various phases in an individual's capacity to adapt effectively to a new culture and has been a central tenet within adjustment and transition research for the past several decades (for a review see Black \& Mendenhall, 1990), despite criticisms concerning empirical support for the UCT (Church, 1982; Furnham \& Bochner, 1986; Ward, Okura, Kennedy, \& Kojima, 1998). Contrary to some researchers' (e.g., Lysgaard, 1955; Oberg, 1960) U-curve propositions that crosscultural adjustment involves four distinct stages ("honeymoon", "culture shock", "adjustment", and "mastery"), Ward and colleagues (Armes \& Ward, 1989; Ward \& Kennedy, 1996; Ward, Okura, Kennedy, \& Kojima, 1998) have found that sociocultural adaptation approximates more of a learning curve with linear improvement over time. Based on this literature, a positive relationship is hypothesised between individuals' length of residence in a new country and sociocultural adaptation.

Length of residence in a host country also increases cultural knowledge (Armes \& Ward, 1989; Torbion, 1982), another situational variable known to facilitate the acquisition 
of behavioural skills in a new cultural environment. Kurman and Ronen-Eilon (2004), for example, found that immigrants' lack of knowledge about Israeli social axioms (e.g., beliefs that guide culturally appropriate behaviours) was related to sociocultural adaptation difficulties. Other researchers (Brown, 2000; Duckitt, 1992) concluded that cultural knowledge was related to positive increases in between-group understanding, and Gong (2003) reported that an individual's tendency to acquire new skills and knowledge was positively associated with both academic and social adjustment. These previous research findings suggest that cultural knowledge will be positively related to sociocultural adaptation.

Learning generalisation-skill-based knowledge or experience that is transferable from one context to another-may be more effective across similar cultural settings. In the acculturation literature, this concept is known as cultural distance, or the objective and subjective differences that exist between an individual's culture of origin and host culture (Babiker, Cox, \& Miller, 1980). Whereas Babiker and colleagues situated cultural distance within a clinical paradigm, other academics (Furnham \& Tresize, 1981) have applied the concept to an intercultural context, and reported that cultural distance exhibited a negative relationship with cross-cultural adjustment. Viewed as a situational factor, additional work on cultural distance has mirrored Furnham and Tresize's findings: Individuals experience greater difficulty with culturally relevant skills acquisition, psychological adjustment, and sociocultural adaptation the more culturally unfamiliar or "distant" their host country is from their country of origin (Chirkov, Lynch, \& Niwa, 2005; Furnham \& Bochner, 1982; Parker \& McEvoy, 1993; Ward \& Chang, 1997). Concordant with these studies, meta-analytic results are expected to show a negative association between cultural distance and sociocultural adaptation.

The culture learning literature has placed importance on the influence of effective intercultural interactions and on sociocultural adaptation more generally. Interpersonal communication skills have been operationalised in terms of language fluency, contact and involvement with nationals, and, to a lesser extent, contact with co-nationals. For example, language fluency is a necessity for learning new cultural behaviours and skills and for increasing culture learning opportunities. Masgoret and Ward (2006) proposed that language proficiency and communication competence comprise the core of sociocultural adaptation processes and act as prerequisites to effective intercultural interactions. Further, language and communication competence have been associated with increased host national contact and friendships (Gullahorn \& Gullahorn, 1966; Perrucci \& Hu, 1995; Quintrell \& Westwood, 
1994; Ward \& Kennedy, 1993b; Zimmerman, 1994). Based on this previous research, language proficiency is anticipated to relate to better sociocultural adaptation.

Although a positive relationship is expected between language proficiency and adaptation, this association is likely to be moderated by contextual factors. In particular, language proficiency is assumed to relate more strongly with sociocultural adaptation in individuals resident in Western countries who are more reliant upon the national languageEnglish, in this instance - to engage with host society members, as opposed to those sojourners in Asian countries or those with multi-national destinations. This hypothesis is predicated upon the assumption that the English-speaking Western countries included in the meta-analysis (e.g., Australia, New Zealand, the United States) may be less likely to accommodate non-English speakers unfamiliar with the language compared to Asian countries such as Singapore, for example, where there is a moderate level of English language proficiency and four official languages: English, Mandarin, and Malay, and Tamil (Education First, 2011). Other Asian countries may have only one national language, but English as a lingua franca may be utilised in urban centres, which can provide sojourning individuals the opportunity to understand and participate in society and may therefore act as a conduit for culture learning.

In addition to foreign language proficiency, early work in the culture learning and cultural competence field centred around intercultural communication vis-à-vis verbal and non-verbal differences, and the influence of these differences on successful social interactions. Although research within the culture learning paradigm later focussed on sociocultural adaptation as an adaptive outcome (Berry, 1997; Sam, 2006; Ward, 1996, 2001a), the importance placed on effective intercultural interactions with members of the receiving society as a way to acquire and master cultural competence has remained. A large breadth of research exists regarding the association between sojourners' interactions with host nationals and increases in culturally appropriate social skills (Bochner, McLeod, \& Lin, 1977; Furnham \& Bochner, 1982; Mak \& Buckingham, 2007; Trice, 2004), cultural knowledge (Bochner, McLeod, \& Lin, 1977; Ong \& Ward, 2005), and observational learning (Black \& Mendenhall, 1990; Black, Mendenhall, \& Oddou, 1991). Based on these findings and Furnham and Bochner's assertion that contact with host members provides learning opportunities necessary for culture-specific skills (1986), meta-analytic findings are anticipated to demonstrate a positive association between host contact and sociocultural adaptation. 
The relationship between host national contact and adaptation may be moderated by how these interactions are operationalised in terms of quality versus quantity of contact, although mixed findings have been reported in this regard. Studies involving intergroup contact theory (Allport, 1954) have shown that both the quantity (e.g., number of outgroup friends) and quality (e.g., closeness of outgroup friendships) of intergroup contact diminishes intergroup prejudice (see also Tropp \& Pettigrew, 2005; Pettigrew \& Tropp, 2006), and sociocultural adaptation research has not methodically differentiated between these two types of involvement. Culture learning theorists have suggested that frequency of contact provides more opportunities for observational learning and cultural instruction, and several studies have corroborated this theoretical rationale (Kagan \& Cohen, 1990; Searle \& Ward, 1990; Ward \& Kennedy, 1992b), whereas other culture learning research has shown that the quality of contact-often framed in terms of social support-a sojourner experiences was also positively associated with effective adaptation, particularly psychological adjustment (Adelman, 1988; Fontaine, 1986; Searle \& Ward, 1990; Stone Feinstein \& Ward, 1990). As these studies suggest, both quantity and quality of contact appear to relate to successful adaptation; however, based on the culture learning premise, it is anticipated that the quantity of contact an individual has with host nationals will have a stronger relationship with sociocultural adaptation than quality of contact.

Some disagreement exists as to whether contact with co-nationals-individuals from a sojourner's home society-hinders or assists successful adaptation. Some research has purported that immigrants and sojourners successfully rely on co-nationals as a source of culture learning and assistance with settling in, presuming the co-nationals have experience in the host country and are able to provide relevant cultural knowledge and skills (Ong \& Ward, 2005). Furthermore, co-national contact may provide recreational or social satisfaction beyond work or academic adaptation, as well as the opportunity to discuss host country issues (Taft, 1977). On the other hand, some academics (Bochner et al., 1977) have suggested that host nationals are more capable of assisting newcomers achieve their adjustment goals than co-nationals, and recent research (Zlobina, Basabe, Paez, \& Furnham, 2006) has found that co-national ties were associated with greater sociocultural adaptation difficulties. Given these contradictory findings, the meta-analysis will examine the direction of the relationship between co-national contact and sociocultural adaptation, but no specific assumptions are made about this association.

Last, the situational variable of perceived discrimination is considered in relation to sociocultural adaptation. The role of perceived discrimination as a predictor of adjustment 
problems has been less investigated in the acculturation literature, but what research has been done on the topic has shown that greater perceptions of discrimination were associated with more adaptation difficulties (Ataca \& Berry, 2002; Ward, 1996; Zlobina, Basabe, Paez, \& Furnham, 2006) and less frequent contact with host nationals (Leong \& Ward, 2000; Ward \& Leong, 2006). It is also of interest to note that the causal direction of the relationship between these two variables has not been established: Weaker intercultural communication skills and cultural competency may lead to an individual's experience of perceived discrimination from the host community, or discrimination experienced by a sojourner may lead to fewer culture learning opportunities and therefore less sociocultural adaptation. Although causality cannot be investigated in a meta-analysis, it is predicted that a significant and negative association between perceived discrimination and sociocultural adaptation will be found.

\section{Psychological Adjustment and Sociocultural Adaptation}

Differences between sociocultural adaptation and psychological adjustment have been previously discussed regarding various antecedents that affect cross-cultural transition. To reiterate, psychological adjustment has been positioned within the stress and coping framework of culture contact, and has been found to be influenced by life changes, coping strategies, social support, and various personality dimensions (Bakker, Van Oudenhoven, \& Van der Zee, 2004; Berno \& Ward, 1998; Searle \& Ward, 1990; Ward \& Kennedy, 1999). Contrastingly, more situational- and learning-based factors involved with the culture learning approach have been shown to predict sociocultural adaptation such as contact with host nationals, previous cross-cultural experience, cultural distance, and language fluency (Ataca \& Berry, 2002; Li \& Gasser, 2005; Masgoret, 2006; Searle \& Ward, 1990; Ward \& Kennedy, 1994, 1999; Zlobina, Basabe, Paez, \& Furnham, 2006). Given the two disparate theoretical backgrounds from which these adaptive outcomes originate and empirical evidence that supports the existence of their contrasting predictive frameworks, it is hypothesised that sociocultural adaptation will be related to but distinct from psychological adjustment with a medium-sized and positive effect size correlation.

Empirical work has also indicated differences between the temporal patterns of psychological and sociocultural adjustment, thus it is suggested that the association between these two adaptive outcomes may be moderated by the length of time individuals spend in their respective host countries. As was previously mentioned, the temporal pattern of sociocultural adaptation resembles a learning curve in that it has been shown to improve over 
time (Ward \& Kennedy, 1995; Ward, Okura, Kennedy, \& Kojima, 1998), with individuals normally experiencing a greater level of social difficulty upon entry to a new culture and a marked decrease in sociocultural adjustment problems later on. In contrast, some studies on psychological adjustment have reported initial difficulties upon entry, followed by an improvement in mental health, and then another subsequent decrease in well-being (Ward \& Kennedy, 1996a). Further, Ward and colleagues (1998) found a non-significant relationship in the magnitude of the psychological-sociocultural adjustment upon participants' entry into the host country (i.e., within 24 hours of arrival) that increased to a significant level after one year. In the current study, a larger association between psychological adjustment and sociocultural adaptation is anticipated in the earlier stages of cultural transition in comparison to later stages of stay. Upon initial entry to a host society, individuals are primarily engaged in coping with a new cultural environment, and as such are managing stress, establishing social support networks, and developing culturally relevant skills to enable more effective functioning during the adjustment process. Both psychological adjustment and sociocultural adaptation improve rapidly during this initial period. As time passes, individuals have more or less learned necessary behavioural repertoires to adapt to their new cultural surroundings and sociocultural adaptation levels off as a result. Contrastingly, psychological adjustment is more variable than sociocultural adaptation, as it is more dependent upon social support and both personal and situational contexts. Therefore, during these later periods of stay, the correlation between sociocultural adaptation and psychological adjustment is likely to be weaker, given the relative stability of sociocultural adaptation and the variability of psychological well-being.

\section{Personality and Individual Differences}

The majority of theoretical and empirical work on cultural competence has traditionally been based within the culture learning framework. As such, much less attention has been paid to the role of personal, compared to situational, factors in the adaptation process.

One rationale for the examination of individual differences and sociocultural adaptation may be found in the substantial amount of theoretical literature on traits considered to be conducive to intercultural effectiveness or cultural competency. Frequently cited individual and personality characteristics thought to enhance effective overseas performance and positive psychological health include open-mindedness, flexibility, and cultural empathy (Abe \& Wiseman, 1983; Church, 1982; Hammer, Gudykunst \& Wiseman, 
1978; Kobrin, 1984; Ratiu, 1983), as well as tolerance to stress (Mendenhall \& Oddou, 1985; Stening \& Hammer, 1989) and ambiguity (Brislin, 1981; Cort \& King, 1979; Locke \& Feinsod, 1982; Maretzki, 1969). The attention paid to personality characteristics and cultural competency throughout the last several decades, as well as the continued use of personality measures in the selection of expatriates and international personnel (Bernardin \& Bownas, 1985; Deller, 1997; Ones \& Viswesvaran, 1999) and in the examination of acculturation processes (Aycan, 2008; Ali, Van der Zee, \& Sanders, 2003; Kealey, 1989; Padilla, Wagatsuma, \& Lindholm, 1985) is indicative of the potentially significant role personality factors play in the explanation and prediction of behaviour.

However, the majority of literature on personality, acculturation, and cross-cultural adjustment has been based on anecdotal evidence and "armchair theorising" (Ward, Leong, \& Low, 2004, p. 137), and what empirical work has been done on the topic has shown inconsistent results regarding the predictive influence of personality on cultural transition. Nonetheless, several researchers have commented on the paucity of and need for empirical research on personality and acculturation (Berry, Poortinga, Breugelmans, Chasiotis, \& Sam, 2011; Kosic, 2006; Ward, Leong, \& Low, 2004), and demands for the consideration of social as well as individual factors associated with acculturation processes have also been voiced (Berry, 1997; Berry, Poortinga, Segall \& Dasen, 2002; Padilla \& Perez, 2003; Schmitz, 2001; Ward, 1996, 2001a). Overall, this literature provides further justification for additional empirical inquiries into the relationship between individual differences and intercultural adjustment.

With this previous literature in mind, the following meta-analysis examined a total of 10 individual differences factors, including both personality and motivation variables, thought to be associated with culture learning and behavioural performance as conceptualised by sociocultural adaptation: These include the Big Five factors of personality (openness, conscientiousness, extraversion, agreeableness, and neuroticism or, conversely, emotional stability); generalised self-efficacy; cross-cultural self-efficacy; cultural empathy; integrative motivation; and an amalgamated motivation factor combining individuals' various motives for moving abroad. These 10 variables are further categorised into a broad individual differences domain, and a more narrowly defined category that relates specifically to intercultural transition and adaptation.

Components of personality within the broader individual differences domain belonging to the Five Factor Model of personality or Big Five (Costa \& McCrae, 1992) will be discussed first. Openness or flexibility, which relates to an individual's willingness to 
experience new behaviours, has been found to predict cross-cultural competence and adjustment. Positioned within the Big Five, various components of openness can be seen to relate specifically to sociocultural adaptation such as an individual's willingness to examine religious, political, or social ideologies, intellectual curiosity, and a readiness or inclination to engage in novel activities. Similarly, conscientiousness - a trait in people considered to be productive, organised, and systematic - has also been investigated in relation to cultural transition in terms of positive expatriate job performance outcomes, more effective transfer of cross-cultural training and learning, and increased self-efficacy (Blume, Ford, Baldwin, \& Huang, 2009; Caprara, Vecchione, Alessandri, Gerbino, \& Barbaranelli, 2011; Kappe \& van der Flier, 2010). Furthermore, research on the Big Factor trait of agreeableness (an individual's tendency to be cooperative and accommodating) has found it to be associated with better social skills, adaptation, and increased social learning opportunities (Sneed, 2002; Tams, 2008). Similar research has replicated this relationship within a cross-cultural context: Ones and Viswesvaran (1997) discovered a relationship between agreeableness and interpersonal aspects of expatriate performance, and Caligiuri (2000) reported that premature international assignment terminations were predicted by lower levels of agreeableness. Based on these studies, positive relationships between openness or flexibility, conscientiousness, agreeableness, and sociocultural adaptation are anticipated.

Conversely, it is predicted that neuroticism, another component of the Big Five, will be related to lower levels of sociocultural adaptation. This hypothesis is based on research that has shown neuroticism to be negatively related to adjustment, job performance, and other domain-specific skill proficiencies (Cheung \& Leung, 1998; Furukawa \& Shibayama, 1993; Tett, Jackson, \& Rothstein, 1991). Further, neuroticism has been linked to the behavioural dimension of cultural intelligence, which involves an individual's ability to learn new behaviours that are culturally relevant (Earley \& Ang, 2003). Emotional stability, as the opposite of neuroticism, has been positioned within the stress and coping framework of acculturation in terms of an individual's ability to manage transition stress and challenges associated with intercultural encounters (Ward, 1996, 2001a). Emotional stability has also been considered an important aspect of cultural competency as is indicated by its inclusion in intercultural measures such as the Multicultural Personality Questionnaire (Van der Zee \& Van Oudenhoven, 2000) and the Cross-Cultural Adaptability Inventory (Kelley \& Meyers, 1995); two instruments that are utilised in the prediction of cross-cultural effectiveness.

The final component of the Big Five Factor Model, extraversion, is expected to have a positive relationship with sociocultural adaptation. Tams (2008), for example, suggested that 
people who exhibit higher degrees of extraversion are more motivated to build relationships, seek feedback, and "create more opportunities for social learning because they engage in more outgoing, gregarious, active, and excitement-seeking behaviours" (p. 199). These sorts of behaviours are advantageous for learning and the acquisition of culturally relevant skills.

Although a positive relationship is expected between extraversion and adaptation, this association is likely to be moderated by context. The relationship between extraversion and sociocultural adaptation is anticipated to be stronger in individuals living in Western, compared to Asian, countries. Support for this supposition can be found in cross-cultural studies of extraversion: Individuals in the United States and Canada, for example, have been found to exhibit higher mean extraversion scores where high levels of extraversion are normative than individuals in Asian countries such as Japan and China (McCrae, 2002). Further, Ward and Chang (1997) found support for normative differences in extraversion with their cultural fit hypothesis, which suggests that extraversion is associated with crosscultural adaptation when levels of extraversion are more similar to host culture norms.

One final factor included in the meta-analysis that has been categorised within the broader individual differences domain is generalised self-efficacy. As a component of Bandura's (1995) social cognitive learning theory, self-efficacy relates to an individual's beliefs regarding their competence and ability to cope with life demands, and to personal agency, the belief that one's motivation and persistence has a direct effect on behavioural outcomes $(1995,1997)$. Bandura has suggested that social cognitive theory is "well suited to elucidate human personal development, adaptation, and change in diverse cultural milieus" (2002, p. 271). He has further proposed that, through personal agency, individuals establish new behaviours, which in turn facilitate effective outcomes that assist them in adapting to diverse cultural environments. Additional research on this topic within intercultural contexts has supported Bandura's theory: Results of a study conducted by Harrison and colleagues (1996) revealed that individuals with high generalised self-efficacy reported significantly greater interaction, work, and general adjustment than those with lower levels of generalised self-efficacy. In another study of young adults from East Germany moving to West Germany after the fall of the Berlin Wall in 1989, Jerusalem and Mittag (1995) also reported that higher generalised self-efficacy was related to more favourable adaptation outcomes. In accordance with this research, generalised self-efficacy is expected to positively relate to higher levels of sociocultural adaptation.

In contrast to generalised self-efficacy, attention will now be given to cross-cultural self-efficacy, one of the variables included in the specific individual differences category. 
Cross-cultural self-efficacy relates to a set of beliefs in one's cross-cultural competence and ability to manage difficulties arising from intercultural situations. Context-specific measures of this form of self-efficacy appear to be warranted (Brenner, 2003), as a growing body of research has shown a positive relationship between it and acculturation in terms of both psychological and sociocultural adjustment (Gong \& Fan, 2006; Long, Yan, Yang, \& Van Oudenhoven, 2009; Mak \& Tran, 2001; Tsang, 2001). In the current study, cross-cultural self-efficacy is operationalised by instruments such as the CQ Motivation Subscale of Cultural Intelligence (Ang, Van Dyne, \& Koh, 2006). The CQ Motivation Subscale entails an individual's drive to function effectively in and to learn more about diverse cultural situations (Ward, Wilson, \& Fischer, 2011). Self-efficacy has been conceptualised as an important aspect of motivational CQ (Ang, Van Dyne, Koh, \& Ng, 2004) as individuals who possess interest and self-confidence in diverse settings are expected to persist through various challenges or difficulties and experience success within these environments. Indeed, motivational CQ has been shown to predict adaptive outcomes such as interaction, work, and general adjustment (Ang et al., 2004; Templer, Tay, \& Chandrasekar, 2006; Ward \& Fischer, 2008) as well as fewer psychological and sociocultural difficulties during cultural transitions (Ward, Fischer, Lam, \& Hall, 2008). This cross-cultural self-efficacy research suggests that the present meta-analysis will produce a positive relationship between this construct and sociocultural adaptation.

Cultural empathy also illustrates the importance of intercultural interactions in the acquisition of behavioural skills and culture-specific knowledge. As a component of intercultural sensitivity, cultural empathy includes aspects of altruism, trust, and sympathy for others, and is characterised by the ability to empathise with the beliefs, emotions, and behaviours of others from differing cultural backgrounds (Ruben, 1976; Van der Zee \& Van Oudenhoven, 2001). It has been suggested that cultural empathy relates to intercultural effectiveness (Arthur \& Bennett, 1995; Hannigan, 1990) and empirically-driven support for this can be seen with Van der Zee and Van Oudenhoven's (2000) incorporation of a cultural empathy scale in their Multicultural Personality Questionnaire, an instrument which was developed in order to examine relevant traits associated with multicultural success. These findings imply that cultural empathy will be positively related to sociocultural adaptation.

As has been noted (Gezentsvey \& Ward, 2008), the concept of motivation has not historically been included in the ABC model of acculturation. To address this concern, two variables specifically related to motivation are included in the current research: Integrative motivation and an amalgamated variable of motives for moving abroad. Integrative 
motivation is a concept that involves an individual's openness and favourable attitudes towards host nationals, and their desire to learn the national language in order to become socially engaged with the host community (Gardner, 1985, 2000). Integrative motivation was initially considered in relation to motivation for second language acquisition and development (Masgoret \& Gardner, 2003); however, consequent research on the topic has indicated that motivation to learn a foreign language and to participate in the host society can be predictive of cultural adjustment (Masgoret, 2006; Masgoret, Bernaus, \& Gardner, 2000) as is an important aspect in the development of both communication and cultural competence (Culhane, 2001; Culhane \& Kimber, 2001).

A second, amalgamated motivation variable was created for the meta-analysis and expected to positively correlate with adaptation. This variable consisted of a group of measures used to examine individuals' motives for moving abroad, engaging in interculturalrelated activities, integrating into a new host society, and socialising with host nationals. Specific cross-cultural transition motivations such as a desire to work overseas (Arthur \& Bennett, 1995; Sinangil \& Ones, 1997; Stone, 1991) and to study abroad (Chirkov, Vansteenkiste, Tao, \& Lynch, 2007) have been found to positively relate with cultural adaptation outcomes. Given this information, both the integrative motivation and the combined motives for moving abroad variables are anticipated to have positive associations with sociocultural adaptation.

\section{Study Overview and Hypotheses}

Within the context of culture learning theory, the current study examined the correlates of cultural competence as assessed by the SCAS. A meta-analytic approach was applied to empirical studies of demographic and situational factors related to learning culturespecific skills, including age, gender, cultural knowledge, length of residence abroad, previous cross-cultural experience, perceived discrimination, host and co-national interactions, cultural distance, and language proficiency. Studies examining the relationship between psychological adjustment and sociocultural adaptation were also included. Research was then extended to individual differences including personality and motivation traits potentially associated with cultural competence. Broader traits including the Big Five (neuroticism, extraversion, agreeableness, conscientiousness and openness/flexibility) and generalised self-efficacy were investigated in addition to more narrowly defined individual differences variables (cultural empathy, cross-cultural self-efficacy, integrative motivation, and an amalgamated motivation variable) believed to relate to intercultural effectiveness. 
Several hypotheses were formulated regarding the expected relationships between these variables and sociocultural adaptation.

1. Positive associations are anticipated between sociocultural adaptation and the situational variables of host and co-national contact, cultural knowledge, previous cross-cultural experience, language ability, and length of host country residence.

2. Negative correlations are hypothesised between sociocultural adaptation and the situational variables of cultural distance and perceived discrimination.

3. A positive, moderate relationship is anticipated between psychological adjustment and sociocultural adaptation.

4. Extending the research inquiry to individual differences, positive relationships between openness, conscientiousness, extraversion, agreeableness, cultural empathy, self-efficacy and motivation variables (e.g., general and cross-cultural self-efficacy, integrative motivation, and a combined motivation variable) and sociocultural adaptation are anticipated.

5. A negative correlation between neuroticism and adaptation is expected.

No specific hypotheses were formulated for the demographic variables (age and gender), as these were not a specific focus of the current study. In terms of potential moderators, the effect of measurement or scale type is examined whenever possible for all relationships, but no specific hypotheses are made. However, two definite moderator hypotheses were formulated:

6. It is anticipated that the language proficiency-adaptation and extraversion-adaptation relationships will be moderated by sojourners' host country destinations; and

7. the psychological-sociocultural adjustment association will be moderated by sojourners' length of residence in their host countries.

\section{Method}

A meta-analysis was conducted to determine the strength of the relationships between variables associated with sociocultural adaptation, a behavioural aspect of cultural competence. As a statistical method that quantifies empirical research findings, metaanalysis (Glass, 1976; Rosenthal, 1984) was chosen due to its potential to provide a broad review of research findings regarding sociocultural adaptation, its ability to allow for comparison of varying strengths and directions of variables across studies, and as a way to 
investigate between-study differences or moderators in participant and study characteristics (Lipsey \& Wilson, 2001).

The following sections provide information about how data were collected and criteria for including studies in the data set. Steps taken in the analysis are then detailed, followed by a discussion of the final sample's characteristics. Homogeneity and heterogeneity of the effect size distributions are also examined, as well as moderator analyses that were conducted on heterogeneous distributions.

\section{Literature Search}

Empirical studies related to sociocultural adaptation as measured by the SCAS were collected via an electronic search using the following databases: PsychInfo, ProQuest, the Web of Science, Dissertations Abstracts International, the Psychological Index, and Psychological Abstracts. Search terms such as "SCAS", "Ward and Kennedy", "adaptation/sociocultural adaptation", "sociocultural adjustment", “acculturation", "difficulty in life", "social behaviour/competencies", and "cultural competencies" were entered into the aforementioned databases (hits $=234)$ and all studies utilising the SCAS were retrieved $(k=$ 104). Any relevant references within these electronic articles were obtained and searched for use of the SCAS. An electronic search of non-English databases (e.g., Anales de Psicologia, Psicothema, and the Directory of Open Access Journals which lists journals published in multiple languages such as French, Spanish, and Portuguese) was performed using translations done by the author of the search terms listed previously (hits $=3$ ).

Electronic mailing lists of psychological organisations were utilised, including Victoria University of Wellington's School of Psychology, the International Association for Cross-Cultural Psychology, the American Psychological Association, the International Academy of Intercultural Research, and the Asian Association of Social Psychology. A request was sent to members of these organisations for any data related to sociocultural adaptation as measured by the SCAS. The request asked for information such as paper identification, country of research, SCAS properties, sample characteristics, and a zero-order correlation $(r)$ table between the SCAS and any demographic, situational, psychological adjustment, or individual differences (personality and motivation) factors examined in the research.

\section{Inclusion Criteria}

Various inclusion criteria were created in order to select research appropriate for the meta-analysis. Only empirical studies utilising the SCAS, published or conducted starting 
with the scale's development (Searle \& Ward, 1990) through April 1, 2011, were included. Study participants were restricted to adults 16 years of age and older. Last, only studies involving variables related to demographic, situational, psychological adjustment, and personality and motivation constructs were included in the current study.

One hundred and four potential studies were found for inclusion in the meta-analysis; however, 24 of these failed to meet the inclusion criteria, leaving a final total of 80 studies for analysis (Appendix A). A total of 21 variables were analysed, falling under the four overarching categories of demographic, situational, psychological adjustment, and individual differences correlates. As was previously mentioned, the demographic category included age and gender $(k=2)$. Psychological adjustment was examined as an additional aspect of crosscultural adaptation $(k=1)$. Contact with host nationals and co-nationals, cultural distance, cultural knowledge, previous cross-cultural experience, language ability, length of residence and perceived discrimination were considered situational variables $(k=8)$. Individual differences variables included the Big Five personality traits, cultural empathy, general and cross-cultural self-efficacy, integrative motivation and an amalgamated motivation factor $(k=$ $10)$.

\section{Coding and Meta-Analytic Procedures}

The study's descriptors (e.g., author, publication year, publication language), SCASspecific information (i.e., number of scale items, internal consistency), and the effect sizes between each of the variables and the SCAS were coded. In the case of longitudinal studies where multiple effect sizes were reported, all time points were averaged together. As the SCAS was scored bi-directionally across studies, the direction of reported effect sizes was reversed when necessary to reflect the relationships between the variables of interest and better sociocultural adaptation.

An effect size is a statistic that determines the quantitative information from each relevant study finding (Lipsey \& Wilson, 2001). These statistics related specifically to the individual procedures and measures used, therefore all metrics were standardised as a value of the same effect size statistic to allow for the equivalent combination, comparison, and interpretation of findings.

Coding findings into standardised effect sizes depends upon the relationship of the variables reported within the studies and the quantitative, reported value of these relationships. As such, effect sizes take various forms such as $r$ or the product-moment 
correlation coefficient (Rosenthal \& DiMatteo, 2001). Effect sizes belonging to this group include Pearson $r$ and $\mathrm{Zr}$, the Fisher transformation of $r$ (Rosenthal \& DiMatteo, 2001).

As the large majority of studies collected for the present meta-analysis included continuous variables that co-varied within a single sample, the $r$ or product-moment correlation coefficient effect size statistic was utilised. The product-moment correlation effect size was also chosen due to its easily understood nature and the fact that it can be converted from other effect sizes (Rosenthal, 1984), including $F$ or $t$ values and standardised beta weights or coefficients $(\beta)$ deriving from simple linear regressions (Reis \& Judd, 2000). Some researchers (Cohen \& Cohen, 1983; Hunter \& Schmidt, 1990) suggest that beta weights originating from regressions with more than one predictor variable cannot be transformed, as $\beta$-values for predictor variables within a multiple regression equation are adjusted for one another. Other researchers have posited that standardised beta weights can be used as effect-size metrics (c.f., Farley, Lehmann, \& Sawyer, 1995; Peterson \& Brown, 2005; Rosenthal \& DiMatteo, 2001). In this meta-analysis, authors of studies that included multiple regression analyses were contacted to obtain a correlation matrix.

Following procedures outlined by Rosenthal (1984), each correlation coefficient was transformed using Fisher's $\mathrm{Zr}$ (Fisher, 1915) and then weighted by their degrees of freedom $(N-3)$ to reflect each values' precision associated with smaller and larger sample sizes. Fisher's $\mathrm{Zr}$ values were converted back to $r$ to issue a weighted average effect size once the mean was calculated.

The correlation coefficients and their corresponding weights were calculated using random-effects analysis procedures. The random-effects approach was utilised because studies in the meta-analysis were only a sampling of research conducted on the topic, and random-effects models account for both between- and within-study differences, which creates more conservative significance tests than fixed-effects models (Field, 2001, 2003; Hedges \& Vevea, 1998; Overton, 1998). A SPSS macro written by David Wilson (2005) was used to obtain basic central tendency statistics. These included the mean effect size, a z-test, homogeneity test, and confidence intervals around the mean effect size. Variability estimates were calculated using $95 \%$ confidence intervals, which estimate variability in the mean correlation.

\section{Homogeneity Analysis and Moderator Analyses}

Homogeneity analyses using a random-effects model were undertaken to examine whether the studies included in the meta-analysis shared similar effect sizes (Hedges \& 
Vevea, 1998). A homogeneous distribution of effect sizes (when the $Q$ statistic is nonsignificant) signifies that the studies collected within the meta-analysis do not vary outside of subject-level sampling error, or that there is no unexplained variability due to external factors that cannot be accounted for by a moderator variable (further discussed below). A heterogeneous distribution of effect sizes (rejecting homogeneity if the $Q$ statistic is significant), on the other hand, indicates that there is significantly more variation than expected by chance that may be due to cross-study differences. The resulting $Q$ statistics indicated that some distributions were heterogeneous, which further necessitated the examination of potential moderators for sources of this variability (Hedges \& Olkin, 1985; Lipsey \& Wilson, 2001). Moderator analyses using the ANOVA analog were performed using Lipsey and Wilson macros (2001). The maximum likelihood method was utilised in order to examine potential differences across mean effect sizes. When interpreting these analyses, $Q_{B}$ pertains to effect size variance due to or moderated by the systematic, betweenstudy differences of measurement type.

\section{Results}

\section{Overview of Included Data Sources}

In total, the meta-analysis was comprised of 80 data sets that met the previously described inclusion criteria. Of these 80 studies, 39 were drawn from peer-reviewed articles. For 9 of these studies, data from correlation matrices provided by the authors were utilised rather than the results published in their articles (see Appendix A). These correlation matrices used the same participant samples described in the corresponding articles; therefore the 9 matrices were coded in the meta-analysis using the articles' study information, participant descriptors, and measurement information (type of measure used, alpha levels). In addition to these data sets, $20 \mathrm{PhD}$ dissertations, 8 studies from book chapters, two underreview papers, one non-peer reviewed article, and one unpublished data set were also included in the meta-analysis.

As the nature of these studies involved adaptation to a new country of residence and comparison of different groups in terms of their sociocultural adaptation, 17 out of 80 of the samples were conducted in two or more countries, hereafter labelled "Multi-National" (see Appendix B and the data set summary in Appendix A). Of the studies conducted solely in one country, data were collected in the United States $(k=19)$, followed by New Zealand $(k=$ 13), Australia $(k=7)$, and Singapore $(k=5)$. Other research countries included Canada, China $(k=3)$, Taiwan, and Israel $(k=2)$. 


\section{Participant Characteristics}

A total of 13,619 participants were recorded in the meta-analysis, with an age range of 17 to 42 and a mean age of 28.01 ( $S D=7.63$ ). There were more females than males; 6,748 and 6,318, respectively. Further, a little over half of the studies' participants were international students $(k=42$ or $52.5 \%)$, followed by combination samples of expatriates, immigrants, sojourners, spouses, and/or volunteers ( $k=9$ or $11.3 \%)$ expatriates only $(k=9$ or $11.3 \%)$; domestic students only ( $k=6$ or $7.9 \%)$; both domestic and international students $(k=$ 5 or $7.5 \%)$; and immigrants $(k=5$ or $6.3 \%)$.

Participants were from varied regions around the globe. Of the 77 studies that reported nationality, 35.4\% involved groups of individuals with various nationalities $(k=28)$. Twenty or $25.3 \%$ of studies reported that respondents came from countries in Asia and South-East Asia (e.g., Japan, China, Malaysia, the Philippines). Participants from Australasia (Australia, New Zealand, and the Pacific Islands) comprised $16.5 \%$ of the studies $(k=13)$, followed by participants from North America (e.g., Canada, the United States, or Mexico; $k=$ $7,8.9 \%)$ and Europe $(k=3,3.8 \%)$.

The majority of research included in the meta-analysis omitted information regarding participants' native languages $(k=63,78.8 \%)$. However, of those data sources that did include such information, English was reported as the most frequent language spoken $(k=7$, $8.8 \%$ ), followed by Chinese (Mandarin or Cantonese) and bilingual speakers of English plus a second language $(k=4 ; 5.0 \%)$, and two native languages of the Philippines, Ilocano and Tagalog $(k=1,1.3 \%$, respectively).

\section{SCAS Characteristics}

The following information pertains specifically to the SCAS as used by the 80 studies included in the meta-analysis. The minimum number of SCAS items included was five, and the maximum number of scale items was $39(\mathrm{M}=22.70, \mathrm{SD}=6.46)$. Over one third of studies included in the meta-analysis reverse coded the SCAS $(k=30$ or 39\%). Overall, internal reliability of the SCAS was high $(\mathrm{M} \alpha=.87)$.

\section{Demographic, Situational, Psychological Adjustment, and Individual Differences Correlates of Sociocultural Adaptation}

A total of 295 correlations were included in the meta-analysis, each of which was categorised into four overarching categories: Demographic, situational, psychological adjustment, and individual differences. In Table 1, the relationships between the variables within these categories and sociocultural adaptation are reported. 
Table 1

Effect Size Correlations Based on the Random-Effects Model

\begin{tabular}{|c|c|c|c|c|c|c|c|}
\hline SCAS Correlates & $\begin{array}{c}\text { Studies } \\
(k)\end{array}$ & $\begin{array}{c}\text { Sample } \\
\text { Size } \\
(N)\end{array}$ & $\begin{array}{l}\text { Effect } \\
\text { Size } \\
(r)\end{array}$ & SE & $\begin{array}{c}-95 \% \\
\text { CI }\end{array}$ & $\begin{array}{c}+95 \% \\
\text { CI }\end{array}$ & $Q$ \\
\hline \multicolumn{8}{|c|}{ Demographic Variables } \\
\hline Age & 27 & 4,350 & .12 & .02 & .09 & .15 & 23.92 \\
\hline Gender & 6 & 1,049 & .19 & .05 & .10 & .29 & $11.21 *$ \\
\hline \multicolumn{8}{|l|}{$\underline{\text { Situational Variables }}$} \\
\hline \multicolumn{8}{|l|}{ Host National } \\
\hline Contact & 23 & 4,127 & .33 & .05 & .23 & .42 & $193.58 *$ \\
\hline Co-National Contact & 5 & 1,232 & .14 & .09 & .04 & .31 & $31.21 *$ \\
\hline Cultural Distance & 20 & 3,640 & -.33 & .05 & -.42 & -.24 & $133.94 *$ \\
\hline Cultural Knowledge & 7 & 876 & .34 & .04 & .26 & .42 & 7.33 \\
\hline Experience & 16 & 2,335 & .17 & .03 & .12 & .22 & 20.02 \\
\hline Language Ability & 32 & 4,523 & .38 & .04 & .30 & .45 & $198.07 *$ \\
\hline $\begin{array}{l}\text { Length of Residence } \\
\text { Perceived }\end{array}$ & 33 & 5,261 & .18 & .03 & .13 & .24 & $104.35^{*}$ \\
\hline Discrimination & 6 & 1,704 & -.50 & .13 & -.75 & -.25 & $116.51 *$ \\
\hline
\end{tabular}

Psychological Adjustment

$\begin{array}{lllllll}53 & 8,529 & .42 & .02 & .38 & .46 & 156.12 *\end{array}$

Individual Differences

Variables

General

Agreeableness

Conscientiousness

$4 \quad 537$

37

$\begin{array}{cccc}.05 & .06 & .25 & 3.68 \\ .04 & .14 & .30 & 6.38 \\ .04 & .21 & .37 & 40.01 * \\ & & & \\ .08 & .06 & .38 & 11.03 * \\ .05 & -.42 & -.24 & 13.94 * \\ .05 & .21 & .39 & 27.81 *\end{array}$

Openness/Flexibility

$6 \quad 868$

868

.16

.22

$14 \quad 2,212 \quad .29$

Generalised Self-

Efficacy

$4 \quad 721$

.29

721

$\begin{array}{ll}.22 & .08\end{array}$

$\begin{array}{ll}. .33 & .05\end{array}$

$\begin{array}{cc}7 & 1,276 \\ 10 & 1,623\end{array}$

.30

Specific

Combined

Motivation Variable

Cross-Cultural Self-

Efficacy

Cultural Empathy

Integrative

Motivation

$.30 \quad .05$

$27.81 *$

\begin{tabular}{cccccccc} 
Motivation & 4 & 1,529 & .26 & .04 & .19 & .34 & 1.40 \\
\hline$k=$ & $N=$ & & & & & \\
& 295 & 50,051 & & & & & \\
\hline
\end{tabular}

Note. Studies in the meta-analysis may have included more than one variable, therefore $295>80$. Significant $Q$ statistics are indicated by $*$ and are significant at the $p \leq .05$ level. 
In the demographic category, the composite effect size was $r=.19$ for gender and $r=$ .12 for age, with females and younger people reporting lower levels of sociocultural adaptation.

Findings from the study supported hypotheses. The results for situational variables included large and medium negative correlations between sociocultural adaptation, perceived discrimination $(r=-.50)$, and cultural distance $(r=-.33)$. The more discrimination participants perceived, the poorer their adaptive outcomes. Participants also experienced lower levels of sociocultural adaptation when their countries of origin were more culturally disparate to their host cultures. In contrast, significant and positive effect sizes were found for the relationships between sociocultural adaptation and co-national contact $(r=.14)$, contact with host nationals $(r=.33)$, and cultural knowledge $(r=.34)$. Greater language proficiency was also related to better sociocultural adaptation, $r=.38$. Effect sizes for the relationship between sociocultural adaptation and previous cross-cultural experience abroad and length of host country residence were similar; $r=.17$ and $r=.18$, respectively. Further, the effect size correlation between psychological adjustment and sociocultural adaptation was $r=.42$.

Individual differences effect sizes ranged from $r=-.33$ to $r=.54$. For variables in the broad individual differences domain, agreeableness was positively correlated with adaptation at $r=.16$, as was conscientiousness $(r=.22)$, extraversion $(r=.29)$, and the openness/flexibility variable $(r=.30)$. Further, a medium, negative effect size was found for the relationship between neuroticism and sociocultural adaptation, $r=-.33$. Generalised selfefficacy and sociocultural adaptation were positively correlated, $r=.22$.

With regard to the more specific culture-centred variables, two large, positive effect sizes were found between cross-cultural self-efficacy, cultural empathy, and sociocultural adaptation. In particular, belief and confidence in one's ability to adapt to a different culture was positively related to adaptation $(r=.47)$, as was participants' empathy, awareness and understanding of their host culture's values and characteristics $(r=.54)$. Last, integrative motivation and the combined motivation variable were found to have similar effect size correlations with sociocultural adaptation ( $r=.26$ and .28 , respectively).

\section{Moderator Analyses Results}

Heterogeneous effect size distributions were investigated for sources of variability to define factors, if any, that systematically affected the effect sizes in the meta-analysis beyond the population parameters. The ANOVA analog was performed in order to examine potential 
differences across mean effect sizes for the categorical variables. As was previously mentioned, the moderating effects of type of scale or psychometric measurement used (where applicable), length of residence in host country, and host country destination were examined.

The moderator variables were defined as follows. The moderating effects of measurement type were investigated in relation to psychological adjustment (scales measuring positive affect such as Diener, Emmons, Larsen, \& Griffin's [1985] Satisfaction with Life Scale versus measures of negative affect such as the Zung Self-Rating Depression Scale [Zung, Richards, \& Short, 1965]); contact with host nationals (quantity of contact versus quality or satisfaction with contact); perceived discrimination (psychometric instruments related specifically to discrimination versus instruments that measured constructs closely related to discrimination such as racism and group permeability); cross-cultural selfefficacy (the CQ Motivation subscale [Ang et al., 2004] versus other intercultural efficacy measures); extraversion (Eysenck's EPQ-R Extraversion Scale [Eysenck, Eysenck, \& Barrett, 1985] versus other extraversion measures including the NEO-FFI [Costa \& McCrae, 1992] and the MPQ Social Initiative subscale [Van der Zee \& Van Oudenhoven, 2000]), and openness/flexibility (measures of flexibility or tolerance of ambiguity versus measures of openness in the Big Five tradition). A second moderator, participants' length of stay in their host countries, was coded into two categories of less and more than two years' stay. A third moderator, participants' host country destinations, was categorised into Eastern culture countries (e.g., China, Japan, Singapore), Western culture countries (e.g., Canada, New Zealand, the United States), and studies that involved multiple destinations.

Despite significant $Q$ statistics, no significant amounts of variance were found for the variables of length of residence, cultural distance, perceived discrimination, and neuroticism. Effect size differences for these variables may have been entirely random, information regarding the moderator values may not have contained enough information to source specific causes of variability, or perhaps certain study level characteristics were not examined by the moderators (Hedges, 1983; Hedges \& Vevea, 1998; Viechtbauer, 2007). However, moderator analyses did uncover sources of variance in the effect sizes for psychological adjustment, cross-cultural self-efficacy, openness/flexibility, host national contact, extraversion, language ability, and cultural empathy (Table 2).

Psychological adjustment. The effect of length of residence was found to partially explain effect size variability in the psychological-sociocultural adjustment relationship, $Q_{B}(1)=9.81, p<.01$. In support of hypotheses, the association between psychological adjustment and sociocultural adaptation was greater for individuals who had resided for two 
years or less in their host country $(r=.50)$ compared to sojourners who had lived for two years or more in a new cultural environment, $r=.37$.

Cross-cultural self-efficacy. Variance in the cross-cultural self-efficacy effect size distribution was found to be moderated by the type of instrument used to measure the construct $\left(Q_{B}[1]=8.25, p<.01\right)$. As was mentioned previously, cross-cultural self-efficacy instruments were grouped into two categories: One category contained those effect sizes based on scores from the CQ Motivation subscale, and the second category involved a combination of other measures of cross-cultural self-efficacy such as Fan and Mak's (1998) cross-cultural self-efficacy instrument. Weaker effect sizes in the relationship between selfefficacy and sociocultural adaptation were found for the CQ Motivation subscale category ( $r$ $=.36)$ than the amalgamated cross-cultural self-efficacy instrument category $(r=.64)$.

Openness/flexibility. The association between openness/flexibility and sociocultural adaptation was moderated by whether or not the studies measured flexibility or openness. Effect sizes derived from flexibility or tolerance of ambiguity scales (e.g., the MSTAT Tolerance of Ambiguity Scale [Mclain, 1993], the MPQ Flexibility subscale) were greater in relationship to participants' sociocultural adaptation $(r=.34)$ than those effect sizes derived from instruments measuring openness such as the NEO-FFI $\left(\mathrm{r}=.17, Q_{B}[1]=4.41, p<.05\right)$.

Host national contact. The effect of measurement type between quantity of host national contact and quality of host national contact was found to partially explain effect size variability, $Q_{B}(1)=5.31, p=.02$. Consistent with hypotheses, the frequency of host national contact and sociocultural adaptation had a larger mean effect size of $r=.37$ compared to the smaller effect size of $r=.18$ for quality of or satisfaction with host national contact and adaptation.

Extraversion. The type of instrument used to measure extraversion explained some variance in effect size heterogeneity: Effect sizes derived from Eysenck's Personality Questionnaire Extraversion Scale were greater in relationship to participants' extraversion and sociocultural adaptation scores $(r=.33)$ than scores from the NEO-FFI $(r=.11)$ and a combined group of other extraversion instruments such as the MPQ Social Initiative subscale $\left(r=.32, Q_{B}[2]=12.07, p<.01\right)$. Further, participants' host countries also contributed to some variation in the relationship between self-reported extraversion and sociocultural adaptation, $Q_{B}(2)=6.13, p<.05$. As hypothesised, a weaker relationship between extraversion and adaptation was found for participants living in Eastern countries than those living in Western countries or groups of participants living in several different countries $(r=$ $.20, .31$, and .35 , respectively). 
Table 2

ANOVA Summary for the Presence of Moderator Variables

Variable

Statistic

(F-Ratio)

$d f \quad$ Average

Effect Size

$(r)$

Psychological Adjustment

Effects of Length of Residence

Between groups

9.81

1

Within groups

16.18

Resident in host country for two years or less

$\begin{array}{lll}8.93 & 4 & .50\end{array}$

Resident in host country for two years or more

7.25

10

.37

Total within groups

25.99

14

\section{Cross-Cultural Self-Efficacy}

Effects of Measurement Type

Between groups

$8.25 \quad 1$

Within groups

6.98

CQ Motivation Subscale

$6.00 \quad 4$

Other cross-cultural efficacy scales

.98

Total within groups

15.23

.36

.64

Openness/Flexibility

Effects of Measurement Type

Between groups

Within groups

Flexibility and tolerance scales

6.22

Openness scales

2.71

.17

Total within groups

13.35

7

Contact with Host Nationals

Effects of Measurement Type

Between groups

5.31

1

Within groups

22.24

Frequency of contact

20.60

15

.37

Satisfaction with contact

1.64

6

.18

Total within groups

27.55

21 


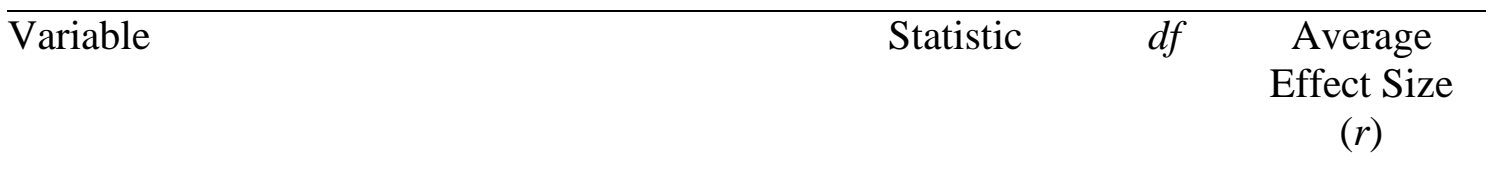

Extraversion

Effects of Measurement Type

Between groups

$12.07 \quad 2$

Within groups

12.98

Eysenck's Personality Questionnaire

Extraversion Scale

$\begin{array}{lll}3.50 & 6 & .33\end{array}$

NEO-FFI

Other extraversion scales including MPQ

Social Initiative

$5.81 \quad 3$

.32

Total within groups

25.06

11

Extraversion

Effects of Destination

Between groups

6.13

2

Within groups

11.37

Eastern destinations

7.05

4

.20

Western destinations

3.86

Multi-national destinations

.47

.35

Total within groups

17.50

1

11

\section{Language Ability}

Effects of Destination

Between groups

Within groups

29.67

Eastern destinations

7.38

4

Western destinations

22.29

24

.40

Total within groups

Note. All effects are significant at $p \leq .05$. 
Language ability. Concordant with predictions, heterogeneity in the effect size distribution of language ability was found to be moderated by destination, $Q_{B}(1)=5.88, p<$ .01 . The relationship between language ability and sociocultural adaptation was weaker for those participants living in Eastern countries than participants living in Western countries, $r=$ .17 and $r=.40$. Multi-national destinations were excluded from this particular analysis due to a lack of studies within this third category.

\section{Discussion}

The first study of this research programme regarding the sociocultural adaptation construct involved a meta-analytic examination of various correlates of cultural competence as assessed by the SCAS. Specifically, two hundred and ninety-five correlations drawn from past studies on the relationships between demographic, situational, psychological adjustment, and individual differences variables and the SCAS were investigated.

\section{Demographic Variables}

Utilising traditional benchmarks (small: $r=.10$; medium: $r=.30$; and large: $r=.50$ ) for effect size interpretation (Cohen, 1988), age and gender were found to have significant but small associations with sociocultural adaptation. Results from other studies involving demographic influences such as gender and age on successful intercultural adjustment have been mixed. Some research has reported a relationship between older expatriates and successful work outcomes and adjustment (Cox, 2004; Hechanova et al., 2003; Mol et al., 2005; Templer, Tay, \& Chandrasekar, 2006), whereas other work has not found such an association (Leong, 2007; Kurman \& Ronen-Eilon, 2004; Nesdale \& Mak, 2003; Searle \& Ward, 1990; Swami, 2008; Zlobina et al., 2006). Similarly, women have been found to be both more (Adler, 1986; Napier \& Taylor; 1995; Hechanova et al., 2003) and less (Caligiuri \& Tung, 1999) successful in new cultural contexts. These diverse findings suggest that, in general, demographic variables may be subject to a range of other contextual factors, such as type and origin of migrating groups and country of destination. Factors such as these would need to be considered carefully in order for meaningful interpretations about cross-cultural adjustment to be made. Overall, results from the current study imply that demographic variables, at least with regard to gender and age, have less of a role to play in the acquisition of culture-specific skills than other adjustment correlates such as situational, psychological adjustment, and individual differences variables. 


\section{Situational Variables}

Based on the established theoretical linkages between culture learning and sociocultural adaptation, predictions concerning situational variables and sociocultural competency as measured by the SCAS were substantiated. Support for these hypotheses includes the association between foreign language ability and sociocultural adaptation: The medium effect size obtained for this correlation highlights recent work by Masgoret and Ward (2006) concerning the central role language proficiency and intercultural communication plays in the adaptation process. Meta-analytic results also support other studies' findings on the relationship between language competence and increased host national contact, friendships, and adjustment (Gullahorn \& Gullahorn, 1966; Noels, Pon, \& Clément, 1996; Quintrell \& Westwood, 1994; Wang \& Mallinckrodt, 2006; Zimmerman, 1994). Further, in line with hypotheses regarding the extent to which sojourners must rely on the national language to operate in the destination country, host country destination partially explained the association between language proficiency and cross-cultural adaptation. Specifically, the relationship between language proficiency and sociocultural adaptation was stronger in Western, compared to Asian, destinations. Because most multilingual Asian countries recognise English as a lingua franca and as a valuable tool for both intercultural and intracultural communication (Honna, 2005; Yano, 2009), these societies may in turn be more accommodating of English-speaking sojourners than Asian-speaking foreigners in Western nations. Overall, further examination of the cultural or national contexts within which immigrants and sojourners acquire new language skills and the relationship between these contexts, language acquisition and intercultural adjustment seem justified and in demand.

Predictions were also confirmed with regard to the situational variables of cultural distance and cultural knowledge. Similar effect sizes between the two variables (cultural distance $r=-.33$ and cultural knowledge $r=.34$ ) and sociocultural competence may suggest more of a mutually dependent relationship between these constructs than has been previously considered. Within the culture learning framework, for example, greater disparities that exist between and individual's home and host cultures would be seen to require greater amounts of effort and learning in order for him or her to function effectively in the host society. Although some researchers have begun to investigate the interrelatedness of cultural knowledge, other adjustment antecedents, and cross-cultural adaptation using a path analysis approach (Swami, Arteche, Chamorro-Premuzic, \& Furnham, 2010), further exploration of potentially causal interrelationships between cultural knowledge, cultural distance, and their 
consequent association with intercultural adjustment would be an interesting avenue for future research.

Further meta-analytic findings supported the expected co-national/host national contact and adaptation relationships, and were congruent with other studies within the culture and social learning paradigms. For example, the socio-contextual model of second language acquisition (Clément, Gardner, \& Smythe, 1980; Noels, Pon, \& Clément, 1996) posits that second language learning and intercultural communication competency are influenced and improved through contact with the host community (Clément, Baker, \& MacIntyre, 2003), and other researchers have found that increased contact with host nationals allows sojourning individuals more opportunities to observe, learn, and model normative social interaction behaviours and cultural rules (Mak \& Buckingham, 2007). Although host national friendships can be more difficult to form (Fontaine, 1986), such relations may assist with more successful long-term adaptation. Conversely, transitioning individuals may initially find it easier and more helpful to initiate contact with co-nationals, especially because conationals who possess appropriate cultural knowledge would ideally be able to provide culture learning assistance (Ward \& Rana-Deuba, 2000). However, the small correlation between co-national and adaptation versus the medium relationship between host nationals and adaptation discovered in the meta-analysis lends credence to the possibility that individuals entering new cultural contexts reliant on contact from co-nationals rather than host nationals may in fact experience greater adjustment difficulties, particularly if they are required to function primarily within the host community (Fontaine, 1986).

Concordant with predictions, moderator analyses also indicated that variance in the host national contact-adaptation relationship may be attributed to measures of quality versus quantity of contact with the host community. Contact with hosts has been cited as one of the more complex antecedents of cross-cultural adaptation (Church, 1982), and the relative importance of the quality and quantity dimensions of contact has been a subject of debate in the acculturation literature (Ward, 2004; Ward \& Rana-Deuba, 2000). The medium correlation between quantity and adaptation versus the small correlation between quality and adaptation goes some way in resolving this debate, and provides a foundation for future studies of cultural competence as well as intergroup contact. To begin to further tease apart these dimensions of contact, however, studies would benefit from a clearer operationalisation of contact, as well as the potential inclusion of both affective (satisfaction with or perhaps another measure for quality of contact) and behavioural (frequency or amount of contact) aspects of host and co-national interactions. 
Last, the study's findings indicate that perceived discrimination, as one of the largest effect sizes of all the meta-analysis variables examined in relation to adaptation, remains a serious challenge to cross-cultural adjustment for some individuals. The mechanisms underpinning this relationship are not clear, however. For example, some research has found that lower levels of intercultural competence predicted greater amounts of perceived discrimination in young immigrants (Neto, 2006; Phinney, Madden, \& Santos, 1998). Other studies have found the opposite relationship, where higher levels of perceived discrimination predicted less social competence (Myrick \& Martorell, 2011). Yet other work has suggested that cross-cultural competence acts as a moderator rather than an antecedent of discrimination (Lee, 2005; Torres \& Rollock, 2007). It seems apparent that further research, and particularly longitudinal studies, concerning the relationship between perceived discrimination and adaptation should be conducted to clarify the processes underlying this relationship.

\section{Psychological Adjustment}

Psychological adjustment and sociocultural adaptation are both adaptive outcomes of cross-cultural adjustment. However, the two constructs are derived from two different theoretical frameworks; stress and coping and culture learning, respectively. Based on the disparate theoretical origins of these domains and previous empirical work demonstrating that the two adaptive outcomes are predicted by different sets of antecedent variables, a moderate association between psychological adjustment and sociocultural adaptation was expected and results confirmed this hypothesis.

The medium-sized correlation found between sociocultural adaptation and psychological adjustment suggests that the SCAS shares some degree of operational overlap with the affective measures of psychological adaptation included in the meta-analysis. For example, as previously noted, Ward and Kennedy's (1999) conceptualisation of the SCAS relates to the degree of self-reported difficulty individuals experience in novel cultural and interpersonal situations. As such, although operationalised as a measure of behavioural competency, the terminology used in the SCAS may potentially evoke emotive rather than behavioural reactions to cross-cultural transitions in a way that could be seen to fall within the stress and coping framework of psychological adjustment. Indeed, as was discussed in Chapter 1, psychological adjustment instruments capture affective processes associated with cross-cultural transitions such as distress, concern, tension, and anxiety (Goldberg, 1972; McNair, Loor, \& Droppleman, 1981; Rohrlich \& Martin, 1991; Schupe, 2007). These meta- 
analytic findings warrant further examination of the conceptual overlap between the SCAS and other indices of psychological adjustment, and more generally the psychometric operationalisation of the SCAS as a behavioural measure of cultural competency.

In terms of moderator analyses, the psychological adjustment and sociocultural adaptation relationship was found to vary as a result of the length of time individuals reported living in their host culture. In particular, the relationship between psychological well-being and sociocultural competence was greater in individuals who had lived in their host countries for two years or less than for those sojourners living abroad for more than two years. Previous research (Armes \& Ward, 1989; Ward \& Kennedy, 1996a; Ward \& Kennedy, 1996b; Ward et al., 1998) supports these results: Participants in these studies experienced the greatest amount of psychological adjustment upon initial arrival to a host culture, which may have been due in part to the extreme degree of life transitions they faced, their potential lack of social support networks, or limited resources. Similarly, results from these studies found that sociocultural adaptation difficulty was also greatest at initial entry, possibly because individuals had less familiarity with and knowledge about the host culture or experienced limited interactions with host nationals. This previous research paired with the meta-analytic findings suggest that, upon arrival, individuals are developing culturally relevant skills to help them function in their new cultural environment, and are also coping with the psychological stress and other emotive reactions experienced as a result of cultural transition. The simultaneous occurrence of these processes could create a strong psychologicalsociocultural adjustment association. However, as is evidenced by the current study, the magnitude of this relationship appears to weaken in later stages of stay. This decrease may be due to the variability that has been found in psychological adjustment over time (Ward \& Kennedy, 1996a) in comparison to findings that suggest sociocultural adaptation levels off once individuals have acquired the appropriate behavioural skills necessary for adapting to their new environments (Ward, Okura, Kennedy, \& Kojima, 1998). Further longitudinal research would be required to further substantiate this temporal relationship between psychological and sociocultural adjustment.

Although untested in the current study, the magnitude of the psychologicalsociocultural adjustment relationship may be affected by a variety of other factors in addition to a sojourner's length of residence in a host society, including acculturation preferences or opportunities for integration into the host culture. Sojourners who interact mainly with conationals or those living in an "expatriate bubble" (Ward, Okura, Kennedy, \& Kojima, 1998, p. 281) who do not rely on the host culture for social interactions may also experience a 
weaker association between psychological well-being and sociocultural competence in comparison to those who operate exclusively in the host culture environment (Ward \& Kennedy, 1996a). Another factor affecting the magnitude of the psychological-sociocultural adaptation association may be attributed to cultural distance. Individuals who experience less cultural distance between their home and host cultures have also reported stronger associations between psychological and sociocultural adjustment (Ward \& Kennedy, 1993b). Overall, the strength of the relationship between sociocultural competency and psychological adjustment cannot be generalised across all individuals or groups in cross-cultural transition: The demographic, situational, and personality factors involved with the sojourner(s) also require careful consideration.

\section{Individual Differences: Personality and Motivation Factors}

Significant relationships between the aforementioned situational variables and adaptation were anticipated due to established theoretical and empirical connections to the culture learning paradigm. However, the current study is the first quantitative, systematic review to provide evidence that individual differences, in terms of both personality and motivation factors, are also an important dimension to consider in the prediction of cultural competence and successful cross-cultural adjustment outcomes.

Broad personality and motivation factors. The meta-analytic results corroborate the expected associations between broad individual differences such as those personality factors belonging to the Big Five model and learning outcomes as measured by the SCAS. Openness, conscientiousness, extraversion, and agreeableness were all significantly and positively related to sociocultural adaptation. This complements other research that has examined how these personality traits relate to culturally relevant outcomes such as increased training ability, cultural awareness, and successful interpersonal relationships and behaviour (Blume, Ford, Baldwin, \& Huang, 2010; Costa \& McCrae, 1992; Kappe \& van de Flier, 2010; Ones \& Viswesvaran, 1997; Sneed, 2002; Tams, 2008). As such, it may be inferred that behavioural characteristics associated with these various personality traits, including intellectual curiosity, willingness to experience new activities, and appreciation or respect of different values and customs, are all favourable and perhaps even necessary attributes required for cross-cultural competence and success.

The negative relationship found between neuroticism/emotional stability and sociocultural adaptation also confirmed predictions. Previous studies have demonstrated negative associations between neuroticism and adjustment, job performance, and the 
behavioural dimension of cultural intelligence (Cheung \& Leung, 1998; Earley \& Ang, 2003; Furukawa \& Shibayama, 1993; Tett, Jackson, \& Rothstein, 1991; Ward, Leong, \& Low, 2004). More generally, neuroticism has been affiliated with skill deficits and occupational performance (Tett, Jackson, \& Rothstein, 1991), and in addition to the behavioural and emotive components of the trait such as hostility, anxiety, and vulnerability, the presumption can easily be made that this personality factor is less than conducive for easy or effective cross-cultural adjustment. Further, emotional stability, as the opposite of neuroticism, has been positioned within the stress and coping framework as it relates to a sojourner's ability to manage stress in intercultural interactions (Duru \& Poyrazli, 2007; Ward, 1996, 2001b). Emotional stability is also an important aspect of cultural competency, as is evidenced by its inclusion in various measures of cross-cultural effectiveness (Kelley \& Meyers, 1995; Van der Zee \& Van Oudenhoven, 2000).

Moderator analyses involving broader individual differences found that cultural context appears to play an important role in the relationship between extraversion and intercultural adjustment. Individuals living in Western countries reported a stronger association between extraversion and sociocultural adaptation compared to those participants residing in Eastern countries and multi-national destinations. Values placed on extraversion and its behavioural expression are known to vary across cultures (Markus \& Kitayama, 1991; McCrae, Costa, \& Yik, 1996) due in part to individualistic-collectivistic differences (Triandis, 1995; Lynn, 1981; Ward et al., 2004) and disparities between personality profiles and host culture norms. These discrepancies or differences in cultural fit highlights the significance of the individual-situation interaction (Mischel, 1984) and the influence of cultural context on interpersonal relations, communication, psychological processes, and intercultural effectiveness (Berry, Kim, Minde, \& Mok, 1987; Berscheid, 1985; Gudykunst \& Hammer, 1988; Taylor \& Moghaddam, 1994; Ting-Toomey \& Chung, 1996; Ward \& Chang, 1997; Ward \& Kennedy, 1993a, 1993b).

Generalised self-efficacy, another factor categorised within the broader individual differences domain, was also found to have a significant and positive relationship with sociocultural adaptation. This was an anticipated finding, as Bandura $(1995,1997)$ purported that an individual's belief in their competence, motivation, and ability to cope with life demands affects behavioural outcomes, and previous empirical work has evidenced a relationship between generalised self-efficacy and more favourable adjustment outcomes (Harrison et al., 1996; Jerusalem \& Mittag, 1995). Individuals who exhibit personal agency are able and willing to take responsibility for their life circumstances, exercise effective 
management of themselves and their surroundings, and show incentive to persist in difficult or troubling situations: These behavioural attributes would prove useful to an individual's successful cross-cultural adjustment. Ultimately, the meta-analytic findings regarding generalised self-efficacy support Bandura's theory and his postulation that the construct may act as a foundation for future investigations of cultural change (Bandura, 2002).

Specific personality and motivation variables. Meta-analytic findings suggest that a distinction can be made between broad individual differences factors and more narrowly defined aspects of personality and motivation in the prediction of sociocultural adaptation. In particular, medium effect size correlations were obtained for broad individual differences in comparison to the large correlations found for more specific aspects of personality and motivation. This broad versus specific distinction may be partially attributed to the fact that, in terms of personality for example, the Big Five traits were derived as a comprehensive and hierarchical organisation of personality characteristics rather than as a psychometric measure of personality specifically developed for acculturation research. As such, they may be less robust predictors of important facets of cross-cultural adjustment such as overseas job performance, multicultural attitudes, interpersonal behaviours, or intercultural effectiveness (Ashton, 1998; Van der Zee \& Van Oudenhoven, 2000). An increased focus on the development of cross-culturally validated instruments that measure more specific personality characteristics such as those included in this meta-analysis may therefore be a worthwhile enterprise (Van der Zee \& Van Oudenhoven, 2000).

Significant associations between measures of integrative motivation, individuals' motives for moving abroad, and cultural competency were also discovered. Generally speaking, motivation is not a widely researched topic in the acculturation literature (Gezentsvey \& Ward, 2008), but these meta-analytic results as well as past work on motivation and cultural adaptation (Chirkov, Vansteenkiste, Tao, \& Lynch, 2007; Sinangil \& Ones, 1997; Stone, 1991) provide convincing evidence for its further consideration in future research.

The large correlations found between cross-cultural self-efficacy, cultural empathy, and sociocultural adaptation are particularly noteworthy. Although some research has investigated the relationship between cross-cultural self-efficacy (Gong \& Fan, 2006; Long, Yan, Yang, \& Van Oudenhoven, 2009; Mak \& Nesdale, 2001; Tsang, 2001; Ward \& Fischer, 2008), cultural empathy (Arthur \& Bennett, 1995; Hannigan, 1990; Van der Zee and Van Oudenhoven, 2000), and adjustment, results from this meta-analysis strongly suggest that 
these variables may in fact influence sojourner adjustment to a much greater extent than other, more traditional variables researched in the past.

\section{Meta-analytic Contributions to Acculturation Theory and Research}

Overall, meta-analytic findings confirm previous culture learning research concerning the influence of situational variables such as host language competence, host and co-national contact, cultural distance, and cultural knowledge on the cross-cultural adjustment of various sojourning groups. Theoretically, this study's findings imply that the culture learning research programme is targeting an appropriate set of variables in the examination of acculturative processes, such as intergroup relations and sojourning groups' successful acquisition of new skills and knowledge in host societies. Results concerning this group of variables also highlight potential avenues of continued theoretical inquiry within the culture learning paradigm, such as further examination of the contextual influences (e.g., familial, intergroup, societal) on adaptation; causal inter-relationships between selected variables such as perceived discrimination and adjustment; differences that exist between quantity and quality of host contact, as well as various operationalisations of contact related to successful adjustment outcomes. In an applied sense, findings from the meta-analysis suggest that cross-cultural training programmes, international student offices, human resource teams, and recruitment agencies alike should continue to highlight the importance of factors such as language fluency, previous cross-cultural experience, and the establishment of meaningful relationships within the host society in relationship to the processes involved with successful cross-cultural transitions.

The current study also makes a novel contribution to the literature by introducing quantitative evidence that individual differences such as personality and motivation factors are important aspects of cross-cultural adaptation and cultural competency. These results also highlight the paucity of individual differences-multicultural competency theories that impedes both conceptual and empirical development in the area (Berry, Poortinga, Breugelmans, Chasiotis, \& Sam, 2011; Kosic, 2006; Ward, Leong, \& Low, 2004). Two potential theories may provide relevant foundations for future use in acculturation and personality research. One is the ecocultural framework developed by Berry and Georgas (Berry, 2001, Berry et al., 1987; Georgas, 1988, 1993), which suggests that individual differences such as personality factors are influenced by ecological and socio-political contexts. The ecocultural framework proposes that such psychological constructs, as manifested by behaviour, may be assessed both at the individual level through various 
psychological methodologies such as psychometric testing, as well as at the country level via population indicators (e.g., means of communication, economic systems, community engagement). Because the ecocultural premise suggests that personality characteristics inform an individual's behaviour, and that human behaviour is in turn shaped by various contextual influences (Georgas, van de Vijver, \& Berry, 2004), this framework would provide an ideal theoretical platform for further examination of the association between individual differences and sociocultural adaptation.

The cognitive-affective theory of personality conveyed by Mischel and Shoda (1995) also provides a foundation for the investigation of individual differences, cross-cultural competency, and adjustment. According to this theory, individuals differ in the extent to which cognitive-affective traits (e.g., beliefs, expectations and encodings) are negotiated and expressed dependent upon psychological features within contextual situations. The proposed influence of personality and other stable affective traits on how an individual processes information and expresses coping behaviours led Mischel and Shoda to suggest that behavioural variability, as a consequence or expression of the more stable components of an individual's personality system, can be relatively predictable. This idea of an established personality system that guides and shapes one's behaviour can easily be applied to differing cultural situations and multicultural contexts. Ultimately, both the ecocultural and cognitiveaffective frameworks would provide sound platforms upon which testable research questions could be based, such as whether or not sojourners who possess key personality traits living within a specific environment will adapt more effectively than those who do not possess these same key traits, or how sojourners differ in the extent to which cognitive-affective traits are negotiated and expressed dependent upon contextual situations.

In terms of empirical development, individual differences have not been widely examined in the culture learning framework. However, what evidence-based work on personality and motivation traits in relation to adjustment exists is encouraging (Ali, Van der Zee, \& Sanders, 2003; Gong \& Fan, 2006; Ones \& Viswesvaran, 1999; Van der Zee \& Van Oudenhoven, 2000; Ward, Wilson, \& Fischer, 2011). This study's findings provide further justification for the establishment of additional, empirical inquiries and development of specific research programmes to further explore the relationship between individual differences and intercultural adjustment.

Advancement of both theoretical and empirical research programmes on individual differences and culture change would also be greatly beneficial in an array of applied settings. For example, an increased focus on both broad and specific individual differences 
variables would aid in employee selection and training for overseas assignments, and predeparture training programmes could begin to incorporate specific techniques related to motivation components such as cross-cultural self-efficacy or cultural empathy. Such techniques based on developing an awareness of and perhaps increasing positive motivations behind cross-cultural transitions could have a substantial impact on an individual's impetus for moving abroad, which could in turn prove beneficial for intercultural communication and successful cross-cultural adaptation.

\section{Limitations, Conclusions, and Future Directions}

Generalisation of these results to all sojourning and immigrant groups and all receiving host societies is not advised due to some limitations of the meta-analysis. First, the meta-analytic approach is as valid as the research it is based upon, therefore any potential methodological issues with the 80 included studies remain potential issues in the quantitative synthesis presented here. In addition, the moderator analyses conducted must be viewed with caution given the relatively small number of studies present within the various study-level categories. Finally, because the analyses pertain to aggregated, higher-order data as opposed to lower-order or individual study-level data, no inferences between these different levels of analysis can be made with any degree of certainty due to ecological fallacy problems (Robinson, 1950; Viechtbauer, 2007).

In sum, the first study of this research programme on the sociocultural adaptation construct examined cultural competence within a culture learning framework, and provided the first comprehensive, quantitative investigation of the relationships between demographic, situational, psychological adjustment, and individual differences factors and sociocultural adaptation. Through an integrated approach to culture competence afforded by metaanalysis, this study confirmed the importance of situational variables related to the culture learning process and acculturation, and represents the first systematic attempt to address how personality and motivation components relate to cultural competence. Overall, the metaanalytic findings highlight the need to consider both personal and situational variables in the acquisition of cultural competence and as predictors of sociocultural adaptation.

This meta-analysis also provides a solid foundation for consequent studies included in this research programme through examination of the factors believed to both assist and hinder cross-cultural competency. The novel emphasis on personality and motivation traits highlights the strong associations that perceived discrimination, various aspects of motivation, and self-efficacy variables appear to share with cultural adaptation despite the 
general underrepresentation of these factors in the current acculturation literature. This gap in the research will in part be addressed in Chapter 4. The current study also brought to attention various psychometric and methodological issues concerning the operationalisation and measurement of the sociocultural adaptation construct using the SCAS. To address these issues, the following chapter summarises a study that sought to revise the SCAS and reframe sociocultural adaptation as a behavioural facet of cultural competency using exploratory factor analysis. 


\section{Chapter Three: Development and Validation of a Revised Measure of Sociocultural Adaptation}

The previous chapter provided a meta-analytic overview of the demographic, situational, psychological adjustment, and individual differences correlates of cultural competency as measured by the SCAS (Searle \& Ward, 1990). One meta-analytic finding of interest involved the correlation $(r=.42)$ between psychological adjustment and sociocultural adaptation. This association may indicate that the SCAS shares some operational or conceptual overlap with the affective measures of psychological adaptation included in the meta-analysis, despite it being a measure of behavioural adaptability situated within the cultural learning paradigm. More specifically, although the SCAS is operationalised as a measure of behavioural competency, the terminology used in the measure may capture emotive rather than behavioural reactions to cross-cultural transitions in a manner that falls more broadly into the area of psychological adjustment and the stress and coping framework of acculturation. To address this possibility, one objective of the present study is to examine the psychometric operationalisation of sociocultural adaptation as a behavioural facet of cultural competency through development of a revised measure of the SCAS.

The current study also addresses additional issues with the SCAS. For example, the SCAS is intended to be a measure of behavioural adjustment yet is worded and scored in terms of "difficulty", which in fact makes it a scale of maladjustment. Further, previous research has found evidence for the existence of various adaptation domains. With this in mind, development of a revised behavioural adaptation scale that captures an overall representation of adaptation as well as specific adaptation domains would prove useful to future research in a variety of ways.

Subsequent to an in-depth presentation of these three issues regarding the current SCAS, the theoretical and methodological origins of the revised sociocultural adaptation measure will be discussed. The theoretical frameworks from which the revised SCAS or SCAS-R is derived are outlined, including social and culture learning, emotion skills and interpersonal communication, cultural intelligence, Berry and Georgas' (Berry, 2001; Georgas, 1988, 1993) ecocultural framework, and domain-specific (task) performance. Presentation of these theoretical frameworks is followed by an overview of the methodological approach taken for the revised measure's development and a discussion of the study's hypotheses. Ultimately, as a prominent cross-cultural adaptation measure, expansion and revision of the SCAS through incorporation of both conceptual and empirical 
research published since its original development would be highly beneficial for the scale's continued reliability and validity, as well as for its utilisation across various fields of research and populations.

\section{Conceptual and Methodological Issues Regarding the SCAS}

The following section describes three main points of concern with regard to the current SCAS as a measure of behavioural cultural competency: (1) Conceptual and operational overlaps between the SCAS and affective measures of psychological adjustment; (2) SCAS terminology concerning adjustment and maladjustment; and (3) the advantages of examining multiple adjustment domains within a behavioural competency context.

Previous theoretical discussion of sociocultural adaptation has placed the construct within a culture learning approach to acculturation, which emphasises the importance of cross-cultural interactions and the acquisition of culture specific interpersonal skills and behaviours in new cultural environments. However, appraisal of the current SCAS raises the possibility that some undue conceptual overlap may exist between it and affective measures of psychological adjustment.

As was previously mentioned, the SCAS refers to the degree of self-reported difficulty a sojourner experiences in new cultural and interpersonal situations. The SCAS prompt reads as follows: "Please indicate how much difficulty you experience in (host country) in each of these areas. Use the following 1 to 5 scale. $1=$ No difficulty; $5=$ Extreme difficulty." Use of the word "difficulty" may capture emotive rather than behavioural responses to cross-cultural transitions in a way that could be seen to fall within the stress and coping framework of psychological adjustment. For example, psychological adjustment indices utilised in studies of sojourner adjustment often involve similar terminology to the SCAS such as "difficulty", as well as other emotive wording including “distress" and "concern" (Goldberg, 1972; McNair, Loor, \& Droppleman, 1981; Rohrlich \& Martin, 1991; Schupe, 2007). Further, acculturative stress, as an aspect of psychological adjustment, has often been defined in terms of difficulties or stressors that arise as a result of the adaptation or acculturative process (Castillo, Conoley, Brossart, \& Quiros, 2007; Joiner \& Walker, 2002, as cited in Rudmin, 2009). In this regard, Rudmin (2009) has suggested that the legitimacy of acculturation instruments is heavily dependent upon explicit and formal definitions of the specific constructs being measured.

Other researchers have also noted the conceptual overlap between the SCAS as a measure of sociocultural adaptation and psychological adjustment. Zhang and Goodson 
(2011) conducted a review of adjustment antecedents, and found that several factors such as English language proficiency, length of residence, and acculturation preferences predicted both sociocultural adaptation and psychological adjustment, and at times with equal strength. These researchers postulated that the aforementioned antecedents shared by both psychological and sociocultural adjustment may be due to an underlying association between the two domains, and, along with other scholars (Furnham \& Erdman, 1995; Oguri \& Gudykunst, 2002), recommended that a review of the adjustment framework as it currently stands be undertaken.

A second, related issue regarding the current SCAS concerns valence. In particular, the SCAS frames sociocultural adaptation in terms of maladjustment rather than adjustment: Higher scores on the SCAS indicate greater difficulty in adapting to a new culture, whereas lower scores indicate less sociocultural adaptation difficulty. The negative valence of the current SCAS could be a point of confusion for researchers and research participants alike, as behavioural adjustment or sociocultural adaptation may be considered as a positive rather than negative acculturative outcome. Indeed, as a way to facilitate interpretation, several researchers who have utilised the SCAS have reverse-scored the items so that higher scores were indicative of positive adaptation (e.g., Gungor \& Bornstein, 2009; Kashima \& Loh, 2006; Li \& Gasser, 2005; Oguri \& Gudykunst, 2002).

A third issue regarding the current SCAS pertains to whether or not sociocultural adaptation can be considered to have multiple domains. Ward and colleagues, as well as the majority of other researchers working with the instrument, have employed total SCAS scale scores as an overall representation of sociocultural adaptation. This conceptualisation mirrors the wider approach taken in the acculturation literature regarding sojourner adjustment, which has largely focussed on an individual's adaptation to the general cultural environment of the host society (Gullahorn \& Gullahorn, 1963; Oberg, 1960; Ruben \& Kealey, 1979; Torbiorn, 1982; Tung, 1983; for reviews see Church, 1982; Stening, 1979). However, researchers have also shown a nascent interest in identifying multiple domains of the SCAS. For example, an early factor analysis of the SCAS (Ward \& Kennedy, 1999) found two domains; one based on cognition and communication that Ward and Kennedy termed Cultural Empathy and Relatedness, and a second domain related to the management of interactions and behaviour titled Impersonal Endeavors and Perils. Further factor analyses on the SCAS have since been conducted: Some researchers have replicated the two-factor structure initially reported by Ward and Kennedy (Moore, 2009; Swagler \& Jome, 2005), and 
others have found various three-factor solutions (Chen, 2010; Daly, 2007; Townsend \& Wan, 2007; Yusoff, 2010).

Although the majority of acculturation research has taken an overall, whole score approach to sociocultural adaptation, examination of multiple dimensions of culture change also warrants attention. The related fields of international business and industrial/organisational psychology have already begun this investigation. For example, Feldman (1976 as cited in Black, 1988) has suggested that cross-cultural adjustment occurs both within and outside the work situation, and other researchers (Brein \& David, 1971; Hawes \& Kealey, 1981) have differentiated between behavioural adjustment that takes place within the general cultural environment (e.g., weather, food) and intercultural interactions with host nationals. Further, based on this previous work, Black and colleagues (Black, Gregersen, \& Mendenhall, 1992; Black, Mendenhall, \& Oddou, 1991; Black \& Stephens, 1989) have provided a widely-utilised, multidimensional conceptualisation of cultural adjustment. Their theoretical and empirical research has extended adjustment into three distinct sociocultural adjustment domains: Adjustment to host national interactions, adaptation to the general environment and culture, and work adjustment. This three-factor approach has been widely accepted theoretically, and also empirically replicated in consequent organisational and international business studies (Caligiuri, 2000; Parker \& McEvoy, 1993; Selmer \& Leung, 2003; Shaffer, Harrison, \& Gilley, 1999; Takeuchi, Yun, \& Tesluk, 2002; Templer, Tay, \& Chandrasekar, 2006).

With this research in mind, further investigation of multiple domains of behavioural adaptation within the acculturation framework would be beneficial. Such work would help delineate the relationships between various antecedents and specific adjustment domains, and would provide more contextual information about what sociocultural areas of adjustment individuals find more challenging than others. Furthermore, examination of multiple behavioural adjustment domains would allow for a more comprehensive understanding of how behavioural competency changes dependent upon differing domains individuals experience in a host society.

\section{Theoretical and Methodological Development of the SCAS-R}

As outlined in the preceding section, a revised measure of sociocultural adaptation, the SCAS-R, was devised to address the conceptual overlap between the original SCAS and affective measures of psychological adjustment, and issues with scale valence in terms of adjustment versus maladjustment. It was also of interest to investigate the potential of 
multiple sociocultural adaptation domains. This last point will be further explored in the following section.

Theoretical development of the SCAS-R: Domain generation. One aim of developing the SCAS-R was to create a measure of behavioural competency comprised of multiple adaptation domains. The SCAS-R was conceived as a measure of self-reported behavioural proficiency in adapting to a novel cultural setting, where the average of SCAS-R items would issue an overall score of behavioural competency. Four sociocultural domains or contexts of self-reported behavioural competency were also proposed: Interpersonal communication, community involvement and personal interests, work or academic performance, and ecological adaptation. The subsequent information summarises the theoretical considerations that were involved with the construction of these four contextual domains.

The theoretical underpinnings of the four suggested domains involve areas of adaptation in which individuals experience challenges with acquiring new behavioural skills. One of the proposed domains, interpersonal communication, was derived from the culture learning approach and conceptualised as an individual's behavioural proficiency or skill in cross-cultural social encounters. Culture learning theory, as has been previously discussed, is one of the most comprehensive models of behavioural adaptation available within the acculturation literature that focusses on an individual's ability to effectively negotiate social or interactive aspects of a new cultural setting (Ward \& Kennedy, 1999). Based on components of this framework, the emphasis placed on interpersonal communication extant in the SCAS has been retained in the SCAS-R with the inclusion of several scale items from the original scale involving verbal communication and social interactions. Specific scale items for this and the other three proposed domains will be further detailed in the Methods section.

An additional theoretical foundation for the proposed interpersonal communication domain originated from Masgoret and Ward's (2006) "concentric circles" representation of foreign language proficiency (Figure 2), in which they conceive of foreign language ability and broader communication competence as two central components of effective social interaction that in turn form the broader construct of sociocultural adaptation. Based on this theoretical positioning of language competency within the culture learning framework, two items measuring an individual's self-reported level of host national language fluency (speaking and understanding) were included in the SCAS-R. 


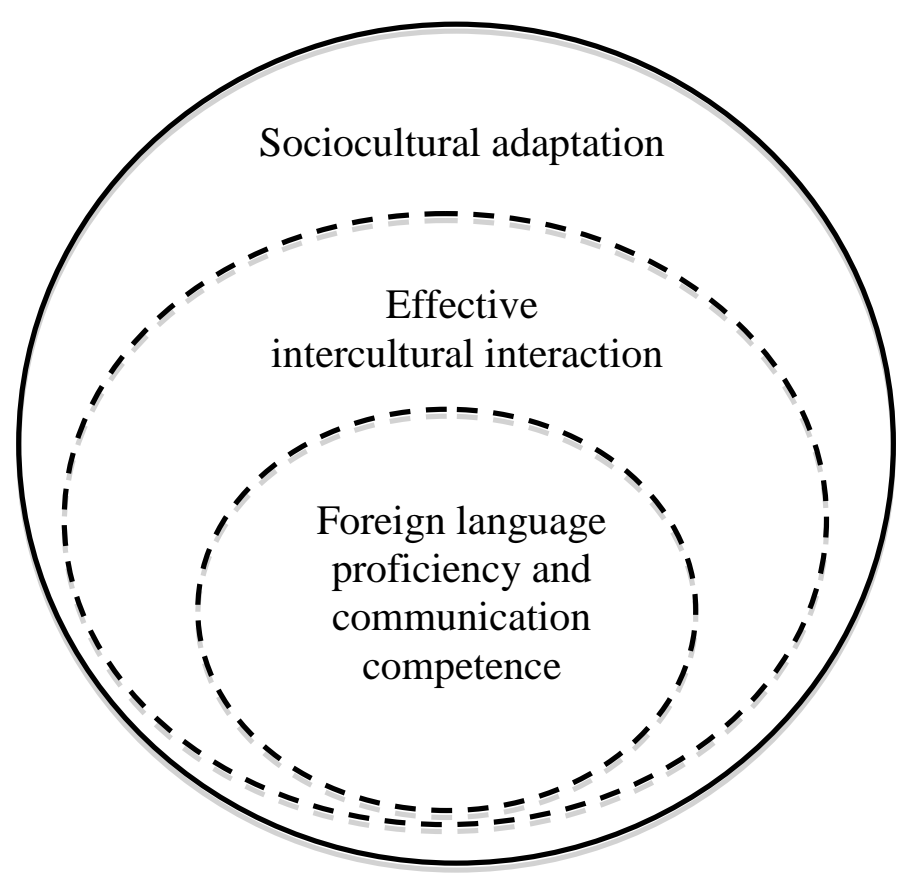

Figure 2. Interactive model of foreign language proficiency, communication competence, effective intercultural interaction and sociocultural adaptation. Reproduced from "The Culture learning approach to acculturation" by A.-M. Masgoret \& C. Ward, 2006, p. 61. In D.L. Sam \& J.W. Berry (Eds.), The Cambridge Handbook of Acculturation Psychology. Copyright 2006 by the Cambridge University Press.

Theoretical development of the proposed interpersonal communication domain was also derived from the social learning paradigm. As has been discussed in Chapter 1, social learning concerns how an individual's learning behaviours are acquired via experience and consequences that occur from actions, observation, and imitation (Bandura, 1977). As a hybrid of cognitive and behavioural theories (Black \& Mendenhall, 1990), the social learning framework offers a wide theoretical base as a way of explaining how individuals learn both intra- and interculturally (Black \& Mendenhall, 1990; Hilgard \& Bower, 1975; Swenson, 1980). Specifically, people experiencing new cultural environments adapt their behaviours, in part, through modelling the behaviours of others as a way to increase positive consequences and reduce negative repercussions (Oberg, 1960; Torbiorn, 1982). Social learning theory further suggests that sojourners retain information about these behaviourconsequence associations, which in turn bolsters their chances of replicating successful behaviours in similar contexts (Bandura, 1977 as cited in Black and Mendenhall, 1990).

Another facet of social interactions and cross-cultural communication included in the proposed interpersonal communication domain that was also briefly discussed in Chapter 1 relates to theories of emotion and emotion-related skills. A large amount of theoretical and 
empirical research has highlighted the importance of this area in successful social interactions, including the ability-based model of emotional intelligence and cultural intelligence.

The ability-based model of emotional intelligence (Mayer \& Salovey, 1993, 1997; Mayer, Salovey, \& Caruso, 2000) purports that emotions are a useful resource for individuals that assist in understanding and navigating social environments. Salovey and Mayer have suggested that individuals vary in their level of emotive skills (e.g., perceiving, understanding, utilising, and managing emotions), and that these individual differences affect wider cognition processes and the recognition of more subtle or complicated interpersonal relationships and cultural artifacts.

Also referenced in the SCAS-R was cultural intelligence or CQ (Ang et al., 2007). The CQ paradigm involves four knowledge components-cognitive, metacognitive, motivational, and behavioural—believed to relate to an individual's capability for the accurate interpretation of unfamiliar verbal and non-verbal cues in cross-cultural contexts (Earley \& Ang, 2003; Elenkov \& Pimentel, 2008; Van Dyne, Ang, \& Koh, 2008). However, only the behavioural CQ component was utilised as a foundation for SCAS-R development, as this factor refers to the enactment of appropriate behaviours within cultural situations.

Moving beyond the proposed intercultural communication domain, Berry and Georgas' (Berry, 2001, Berry et al., 1986; Georgas, 1988, 1993) ecocultural framework was broadly applied to two of the proposed sociocultural domains; an individual's behavioural adaptation to the general ecological environment, and a person's ability to become involved with the community and maintain personal interests. Berry and Georgas' ecocultural approach considers cultural and psychological human diversity as collective and individual adaptations to contexts that include ecology, education, the economy, mass communications, and population. Berry and colleagues (1986) later framed this approach within a crosscultural framework, employing these ecological and social political contexts as independent variables in order to study their influence on various psychological constructs. Georgas (1988, 1993) further differentiated elements of the ecocultural framework into three main sociocultural domains: (1) Societal and institutional organisation; (2) community or group participation and bonding; and (3) family. The two proposed sociocultural domains of ecology and community involvement/personal interests are a loose reflection of this theoretical perspective, but are not specifically situated within Berry and Georgas' ecocultural premise. 
A final, potential domain examined for inclusion in the revised SCAS involved an individual's ability to perform behavioural tasks (a combination of abilities, skills, and knowledge that shape role-prescribed behaviours [Campbell, 1999]) in domain-specific settings such as academic or work situations. These two contextual settings were specifically chosen for inclusion in the SCAS-R because sojourners commonly relocate as a result of either study (e.g., international students) or employment (e.g., expatriates) opportunities. Various research was drawn on to develop items related to this context-specific domain, such as Pulako, Arad, Donovan, and Plamondon's (2000) adaptive performance paradigm, Ward and colleagues (Berno \& Ward, 2003; Ward \& Masgoret, 2004) academic adjustment measurement, Black and Stephen's (1989) work adjustment domain, and theoretical work by Mak and colleagues used to develop the ExcelL programme, a behavioural competence training course previously mentioned in Chapter 1 (Mak \& Barker, 2004; Mak \& Buckingham, 2007; Mak et al., 1998).

First, Pulakos and colleagues (2000) defined a cultural dimension of adaptive performance - the performance of observable and measurable behaviours based on an individual's level of proficiency - that involves cultural adaptability within a work or academic role. This adaptability entails understanding the needs and values of other groups and organisations, and an individual's ability to willingly adjust behaviour out of respect for others' values and customs. Further, Ward and associates (Berno \& Ward, 2003; Ward \& Masgoret, 2004) have considered domain-specific adaptive performance in reference to difficulties international students have experienced in academic settings within their host societies. In addition, as was previously mentioned, research by Black and colleagues outlined a three-domain approach to adaptation that includes work adjustment. Last, the theoretical underpinnings of the ExcelL programme-a course designed to improve international students' behavioural competencies in various social contexts-were also drawn upon in conceptualising the context-specific domain. Overall, inclusion of this potential performance domain within the SCAS-R is advantageous due to its generalisability to expatriate, working migrant, and student populations.

Creation of a revised sociocultural adaptation measure with multiple adjustment domains has several advantages. A large amount of evidence has suggested the presence of various domains of adaptation an individual experiences when traversing cultures, therefore development of an instrument with multiple adaptation domains would potentially allow for more specific investigation and application of the sociocultural adaptation construct. 
Methodological development of the SCAS-R. Methodological and empirical development of a psychometric instrument begins with the construct's operational definition; operations or procedures that delineate the parameters of specific psychological phenomena (Ratner, 1997). The operational definition of sociocultural adaptation as measured by the SCAS-R was previously provided: The SCAS-R, as a measure of sociocultural adaptation, is conceptualised as the degree of behavioural competency an individual reports in adapting to a new cultural environment. Once an operational definition is developed, theoretical rationales must then be provided to validate why the content of an instrument is believed to accurately represent the construct. Such rationales were examined in the previous section in relation to theories of culture and social learning, emotion-related skills, emotional intelligence, theories of ecocultural adaptability, and adaptive performance. However, the value of these theoretical perspectives in terms of their association with sociocultural adaptation can only be determined through methodological investigation of behavioural competency.

The most common approach to the methodological construction of a psychometric instrument relates to the idea of construct validity, including face and convergent validity. Face validity, or prima facie, involves how well a scale item is judged to reflect the construct being measured. In the case of the SCAS-R, face validity relates to the degree to which each of the scale items reflects the idea of an individual's behavioural competency in adapting to a new cultural environment.

Another form of construct validity, convergent or criterion validity, refers to how well a newly developed measure correlates with measures with which it is theoretically associated. Several instruments were selected to examine the criterion validity of the SCAS-R, including measures of cross-cultural adaptation, non-verbal communication, behavioural and social skills, and psychological adjustment. Two measures of cross-cultural adaptation were selected, the original SCAS and Black and Gregersen's (1990) Subjective Adjustment Scale (SAS), as both scales involve adjustment to relationships and social interactions with host nationals. Three behavioural and social skills scales were also included as criterion measures: (1) The behavioural component of the Cultural Intelligence Scale or CQB (Ang et al., 2007); (2) Galchencko and van de Vijver's (2007) measurement of an individual's adaptation behaviours in a host country (SAB); and (3) the Social Skills Inventory or SSI (Riggio, 1986). Similar to various SCAS-R scale items, these measures assess different components of social and behavioural ability. The CQB, for example, measures overall behavioural effectiveness within novel cultural environments; the SAB is a scale that determines how often an individual engages in various behaviours in their host country such 
as listening to host national music or celebrating national holidays; and the SSI assesses an individual's skills in sending, receiving, and controlling interpersonal communication displays.

Last, as has been mentioned, a long-standing distinction exists between sociocultural adaptation and psychological adjustment (Ward, Bochner, \& Furnham, 2001). Research has suggested that these two dimensions of cross-cultural adaptation are interrelated (Kennedy, 2000; Ward \& Kennedy, 1999), therefore two measurements of psychological adjustment were included as criterion measures of the SCAS-R: The Satisfaction with Life Scale or SWLS (Diener, Emmons, Larsen, \& Griffin, 1985) and the Zung Self-Rating Depression Scale or ZSDS (Zung, Richards, \& Short, 1965). The former relates to an individual's selfreported life satisfaction, and the latter involves psychological, physiological, and affective components of depression. Further psychometric information about these criterion measures will be provided in the Methods section.

Once an item pool was generated and criterion instruments were selected, exploratory factor analysis (EFA) was employed as a method by which to examine the factor structure and internal consistency of the SCAS-R. Exploratory factor analysis, first developed by Spearman (as cited in Fabrigar, Wegener, MacCullum, \& Strahan, 1999), is an analytic technique widely utilised in psychological research that aims to identify and reduce a group of interrelated variables to a smaller number of latent factors (Fabrigar et al., 1999; Tinsley \& Tinsley, 1987).

\section{Study Overview and Hypotheses}

There were several aims of the present study. One central research objective sought to more solidly position the SCAS-R within the culture learning framework as a self-report measure of behavioural competency through removal of potentially emotive or affective terminology extant in the original scale. Other study aims were to examine the revised scale's factor structure through the inclusion of potential adaptation domains, to demonstrate evidence of its internal reliability, and to evaluate the new measure's construct validity.

With the study's first objective in mind, it was hypothesised that correlations between the SCAS-R and the two included measures of psychological adjustment would be smaller than the psychological adjustment-SCAS association found in Study $1(r=.42)$ :

1. The SCAS-R will have a positive correlation of $r \leq .42$ with the SWLS.

2. The SCAS-R will have a negative association of $r \leq .42$ with the ZSDS. 
In addition, an EFA of the SCAS-R item pool explored four provisional sociocultural adaptation domains including ecological adaptation, community involvement and personal interests, communication and social interactions, and adaptive performance in a work/academic setting. Several hypotheses were formulated in this regard.

3. Results of the factor analysis will reveal the existence of four potential SCAS-R domains, and are anticipated to confirm contrasting relational patterns between these domains and other study indices and subscales. Specific predictions relating to these subscales are outlined below in hypotheses 4-6.

4. If an interpersonal communication domain is established, this subscale is expected to moderately and positively correlate with the SAS Interaction Adjustment subscale, the SSI, and the CQB.

5. The academic/work performance domain, if supported by factor analysis results, is anticipated to have a large and positive correlation with the SAS Work Adjustment subscale.

6. Dependent on the discovery of a community and personal interests domain, this potential subscale is hypothesised to have moderate, positive correlations with the SAB scale.

Further, correlations between the SCAS-R and various criterion measures will be examined with regard to the revised scale's construct validity. As such, the following hypotheses were developed:

7. Construct validity of the SCAS-R will be supported through:

a) A negative, large association with the original SCAS;

b) a positive, moderate relationship with Black and Gregerson's SAS;

c) positive, moderate correlations with other measures of social and behavioural skills including the Social Skills Inventory or SSI, the behavioural component of the CQB scale, and Galchenko and van de Vijver's host country adaptation scale $(\mathrm{SAB})$. 


\section{Method}

\section{Participants and Procedure}

Various international organisations such as international student university offices, embassies and consulates, non-profit immigrant and refugee groups, online forums, and community groups were contacted in New Zealand and internationally. E-mails were sent asking these associations to provide affiliated individuals with information about the research via an e-mail, listserv, newsletter, or mailing list. Participants were not directly contacted by the researcher. E-mails to these organisations outlined the following inclusion criteria: Eligible participants were to be international students, expatriates, immigrants, and refugees aged 16 years and older living in a host country (e.g., not their country of origin) for five years or less at the time of the study. This time period was selected due to previous literature that has suggested that behavioural adaptation occurs during initial entry into a host society and levels off gradually as new cultural skills are acquired (Furnham \& Bochner, 1986; Ward \& Kennedy, 1999; Ward, Okura, Kennedy, \& Kojima, 1998; Ward \& Rana-Deuba, 1999). A link to the survey was also included in the e-mail. A total of 432 responses were collected initially, but exclusion of those individuals who failed to meet the inclusion criteria lead to a final participant count of 316. At the end of the survey, participants were offered the chance to participate in an anonymous lucky draw to win an iPod or gift voucher. The survey was open from 17 March 2010 to 1 July 2010, and preliminary results were posted on the Centre for Applied Cross-Cultural Research's website in October 2010.

The 316 eligible respondents who provided demographic information (216 women, 87 men, and four missing; $\mathrm{M}_{\mathrm{age}}=26.89, \mathrm{SD}=9.07$; age range 16-56 years) were self-reported expatriates $(N=64)$, immigrants $(N=89)$, international students $(N=144)$, refugees $(N=8)$, and spouses of expatriates or immigrants who had not yet secured permanent residency in their respective host countries $(N=4)$. The majority of participants were American $(N=58)$, New Zealander $(N=45)$, Malaysian $(N=38)$, and British $(N=16)$, followed by groups of participants of 14 and less representing over 55 nationalities and dual nationalities. When considering host country of residence, $67 \%$ or 193 participants were living in New Zealand, followed by $6.6 \%$ in Canada $(N=19)$, and 9 participants or $3.1 \%$ in the United States. A complete list of participants by host country is provided in Appendix C. Of the 302 participants who completed the demographic questionnaire items regarding languages spoken, 69\% spoke at least one additional language, 35\% spoke two additional languages, and $16 \%$ reported speaking three or more additional languages. Participants reported having 
lived in their respective host countries for an average of 1 year $(M=12.06$ months, $\mathrm{SD}=$ 11.35), and most expected to remain for one year or less (31.1\%), followed by three years or more $(26.5 \%)$, between one and three years $(25.9 \%)$, and permanently $(16.5 \%)$. Last, of the 316 individuals who completed the survey, over half were full-time students $(N=182)$. Seventy-seven participants were full-time employees, 18 were part-time students, 7 were part-time employees, and 31 fell into none of the aforementioned categories.

\section{Materials}

The survey included the following sections and information (Appendix D). First, an overview of the research project, inclusion criteria, and an agreement of anonymity and participation consent were provided. A demographic section followed to gather participants' personal information (e.g., gender, age, host country, English language proficiency). An item pool for construction of the SCAS-R was also included, along with criterion measures of sociocultural adaptation, depression, life satisfaction, and behavioural and social skills.

\section{Item Pool Generation}

Sociocultural adaptation is an outcome of culture learning that is generally defined as the process of acquiring specific skills to facilitate successful engagement with a new cultural environment (Ward \& Kennedy, 1999). Development of a 54-item pool and four provisional sociocultural adaptation domains therefore began with this definition and extended into other applied and theoretical research areas that were previously described. The theories and previous research used as a foundation for the SCAS-R item pool will therefore only be briefly reiterated below.

As sociocultural adaptation focusses on behavioural adaptability or cultural competence, a Likert scale was used to reflect the degree of competency an individual reports (endpoints $1=$ Not at all competent $5=$ Extremely competent). Historically, sociocultural adaptation has emphasised effective interpersonal communication and the absence and/or acquisition of social skills in new cultural contexts (Furnham \& Bochner, 1982a; Searle \& Ward, 1990; Trower, Bryant, \& Argyle, 1978; Ward \& Kennedy, 1999); as such, 14 items from the original SCAS were retained in the revised measure, including items such as "Understanding jokes and humour" and "Communicating with people of a different ethnic group". These items were tentatively placed in the Communication and Social Interaction domain.

Development of the interpersonal communication domain was also based on social learning theory (Bandura, 1977) and a "concentric circles" representation of foreign language 
proficiency developed by Masgoret and Ward (2006). Specifically, two language-related items were included to assess ability with the host language, "Understanding and speaking [host language]" and "Reading and writing [host language]". This communication domain also referenced the ability-based model of emotional intelligence (Mayer, Salovey, \& Caruso, 2000) concerning an individual's ability to process and express emotive information, and the cultural intelligence paradigm (Earley \& Ang, 2003; Elenkov \& Pimentel, 2008; Van Dyne, Ang, \& Koh, 2008). Eight items (e.g., "Changing my verbal behaviour [e.g., accent, tone] in a culturally appropriate manner") were influenced by these emotion-related frameworks.

A further 20 items and two proposed domains - one involving ecological adaptation (10 items) and the second community involvement and the maintenance of personal interests (10 items) — originated from Berry and colleagues (Berry, 2001; Berry et al., 1986) and Georgas' $(1988,1993)$ ecocultural framework. Items such as "Adapting to the noise level in my neighbourhood", "Adapting to the population density", and "Attending or participating in community activities" partially reflect this ecocultural premise.

Ten items for the potential task or contextual performance domain were developed to reflect an individual's behavioural competence within an academic or work context. These items (e.g., "Gaining feedback from other students/work colleagues to help improve my performance") were adapted from a measure of academic adjustment (Berno \& Ward, 2003; Ward \& Masgoret, 2004), the ExcelL programme (Mak, Westwood, Barker, \& Ishiyama, 1998), and the Job Adjustment domain developed by Black and colleagues (Black, Mendenhall, \& Oddou, 1991; Black \& Gregersen, 1990).

After the item pool (54 items total) had been generated, small groups of postgraduate students were formed to discuss the content, face validity, and parsimony of the scale. Individual items were revised and again examined until consensus regarding these scale considerations was reached. The items were then piloted on 10 different postgraduates who indicated that each item was easy to comprehend.

Criterion measures of adaptation. Adaptation was assessed with two criterion measures of adaptation; the original SCAS and Black and Gregersen's (1990) Subjective Adjustment Scale (SAS). A 23-item version of the original SCAS was included to measure the amount of difficulty an individual has experienced in various situations (e.g., making friends, getting used to local food). For each item, individuals respond to a five-point rating scale (endpoints 1 = No difficulty, 5 = Extreme difficulty) where higher scores reflect more sociocultural adaptation problems. The meta-analysis conducted in Study 1 found the SCAS to have an overall Cronbach's alpha of .87 in the 80 studies included in the meta-analysis, 
and the SCAS has shown acceptable validity and reliability in cross-cultural samples (Ward $\&$ Kennedy, 1999). The scale's internal consistency in this study was high $(\alpha=.92)$. The 13item SAS and its three sub-scales was utilised as an additional criterion measure. The SAS includes questions regarding an individual's general adjustment (6 items; e.g., living conditions in general, cost of living), interacting with host nationals (4 items; e.g., interacting with hosts on a day-to-day basis), and work adjustment (3 items; e.g., performance standards and expectations). Higher scores (from $1=$ Not adjusted at all to $7=$ Completely adjusted) signify greater adjustment. The SAS was found to have good internal consistency $(\alpha=.93)$ as well as its three subscales; General Adjustment, Interaction Adjustment, and Work Adjustment ( $\alpha \mathrm{s}=.92, .94$, and .94 , respectively), which is consistent with previous literature (Black \& Stephens, 1989; Selmer, 2005).

Psychological adjustment measures. Psychological adjustment was assessed with the Satisfaction with Life Scale or SWLS (Diener, Emmons, Larsen, \& Griffin, 1985) and the Zung Self-Rating Depression Scale or ZSDS (Zung, 1965). The SWLS consists of five items such as "I am satisfied with my life" that measure life satisfaction on a Likert scale of $1=$ Strongly disagree to $5=$ Strongly agree. This version of the SWLS has been utilised in 13 countries across 25 ethnic groups and has shown good validity and reliability (Diener et al., 1985; Pavot \& Diener, 1993, 2008). The scale's internal consistency in this study was $\alpha=$ .87. The second psychological adjustment instrument utilised was a 19-item version of the ZSDS, which examines affective, physiological, and psychological components of depression. Individuals are asked to indicate on a 5-point scale how frequently (endpoints 1 $=$ A little of the time, $5=$ Most of the time) they experience various depression components (e.g., "I have trouble sleeping at night"), where higher scores represent greater depression. The ZSDS has been used in cross-cultural studies (Zung, Richards \& Short, 1969; Zung, 1972) and has proven to be a reliable instrument with high internal consistency in Ward and colleagues' research with multinational samples of sojourners (Ward \& Kennedy, 1993a; Ward, Leong, \& Low, 2004). In this study, Cronbach's alpha was .81.

Behavioural and social skills. Two behavioural measures were included in the survey to further ascertain the construct validity of the SCAS-R. The first was the behavioural component of the Cultural Intelligence Scale or CQB (5 items). Developed by Ang and colleagues (2007), the CQB examines nonverbal and verbal behavioural capabilities thought to relate to a person's ability to function effectively in various cultural settings on a scale of 1 (Strongly disagree) to 7 (Strongly agree). The CQB has been shown to have acceptable internal consistency (Ang et al., 2007; Van Dyne, Ang, \& Koh, 2008) and 
Cronbach's alpha was .90 in the current study. The second 8-item behavioural measure adapted from Galchenko and van de Vijver (2007) examines sociocultural adaptation in participants' host countries (SAB). This scale assesses how often participants speak their host country language, listen to music from their host country, and participate in host country celebrations (endpoints 1 = Never, 7 = Daily or almost daily). Cronbach's alpha was acceptable $(\alpha=.76)$, which is consistent with previous literature (Galchenko \& van de Vijver, 2007; Suanet \& van de Vijver, 2009).

A social skills instrument, the Social Skills Inventory or SSI (Riggio, 1986), was also employed as a criterion measure. A 23 -item shortened version of the original 90 -item scale provided by the instrument's author measured basic social skills believed to underlie social competence through verbal and non-verbal communication (e.g., "I can control my emotions when I need to"). Some of the items were modified to reduce cultural bias. For example, colloquialisms such as "what makes people tick" were changed, and items regarding controlling emotions were removed due to evidence that cultural variations in how emotions are displayed and decoded serve as norms for the appropriateness of judging and displaying emotions (Ekman, 1972; Matsumoto, 1990; Matsumoto, Kudoh, Scherer, \& Wallbott, 1988). The SSI has been found to have high internal consistency in previous studies (DiTommaso, Brannen-McNulty, Ross, \& Burgess, 2003; Riggio, 1986) as well as within this study ( $\alpha=$ $.81)$.

\section{Results}

The main objectives of the present research were to develop a new version of the SCAS, explore its factor structure and potential adaptation domains, demonstrate evidence of its internal consistency, and to evaluate the revised scale's construct validity. To pursue these goals, an exploratory factor analysis was first conducted, followed by a series of correlations.

\section{Exploratory Factor Analysis}

The following steps were followed for conducting an exploratory factor analysis (EFA). First, the data matrix was examined to ensure all variables to be factor analysed were administered to all the participants (Tinsley \& Tinsley, 1987). Second, an adequate sample size of 316 was confirmed, as Tabachnick and Fidell (2001) and others (Comrey \& Lee, 1992) suggest that 300 or more participants represent an acceptable sample size. Subject-toitem ratio was also examined. According to various researchers (Gorsuch, 1983; Hatcher, 
1994; Osborne \& Costello, 2004) a minimum subject-to-item ratio of at least 5:1 in EFA is considered satisfactory. Internal consistency was then checked using inter-item correlations (Field, 2005). Any items correlating too highly (over $r=.90$ ) with any other item were removed to avoid issues with multicollinearity (Field, 2005; Hutcheson \& Sofroniou, 1999). Descriptives were run on the remaining items; those items with high means (indicating a ceiling effect), small standard deviations, and skewed box plots were omitted (Clark \& Watson, 1995; Tabachnick \& Fidell, 2001). A correlation matrix of the remaining 21 items was produced and examined for multicollinearity and sampling adequacy. The determinant of 3.63E-005 was greater than the suggested minimum of .0001, which suggested multicollinearity was not present. Further, results of the Kaiser-Meyer-Oklin measure of sampling adequacy $(\mathrm{KMO}=.89)$, and a test of the average item communalities $(\mathrm{M}=.64)$ found that the 21-item SCAS-R could be factor analysed (Hutcheson \& Sofroniou, 1999; MacCallum, Widaman, Zhang, \& Hong, 1999; Tabachnick \& Fidell, 2001). Principal component analysis (PCA) was chosen as the communality estimate and extraction method to employ, as the aim of the analysis was to reduce the original item pool into a smaller number of items while still retaining the same information (Fabrigar, Wegener, MacCallum, \& Strahan, 1999; Tinsley \& Tinsley, 1987).

After extraction, three techniques were employed to determine the number of factors to retain for rotation (Costello \& Osbourne, 2005; Tabachnick \& Fidell, 2001): (1) GuttmanKaiser's eigenvalue over 1 rule; (2) Cattell's scree plot; and (3) the percent of variance accounted for by the factor solution compared to randomly-generated eigenvalues (parallel analysis, see Horn, 1965) using Monte Carlo PCA for Parallel Analysis software (Watkins, 2000).

Using these three techniques, five components or factors were retained for rotation. First, the scree plot's point of inflection tailed off after approximately the fifth factor (Figure 3). Second, the eigenvalues of these five factors all were above 1 and explained $64 \%$ of the common variance, with the majority of this variance (36\%) attributed to the first factor (Table 3). Last, parallel analysis with 21 variables, 316 subjects, and 50 replications revealed that a four- rather than five-factor solution best explained the variance when eigenvalues from the data set were compared against eigenvalues generated from a random data set based on the same number of items and sample size: Factor 1, 7.57 versus 1.51; Factor 2, 2.01 versus 1.42; Factor 3, 1.54 versus 1.35, Factor 4, 1.28 versus 1.27, and Factor 5, 1.09 versus 1.24. Although parallel analysis explained only four factors, evidence from the Kaiser eigenvalue rule and the amount of variance explained by the fifth factor alone (5\%) led to the decision to 
retain all five factors. Conceptual rationale for retaining the fifth factor will be provided in the next section.

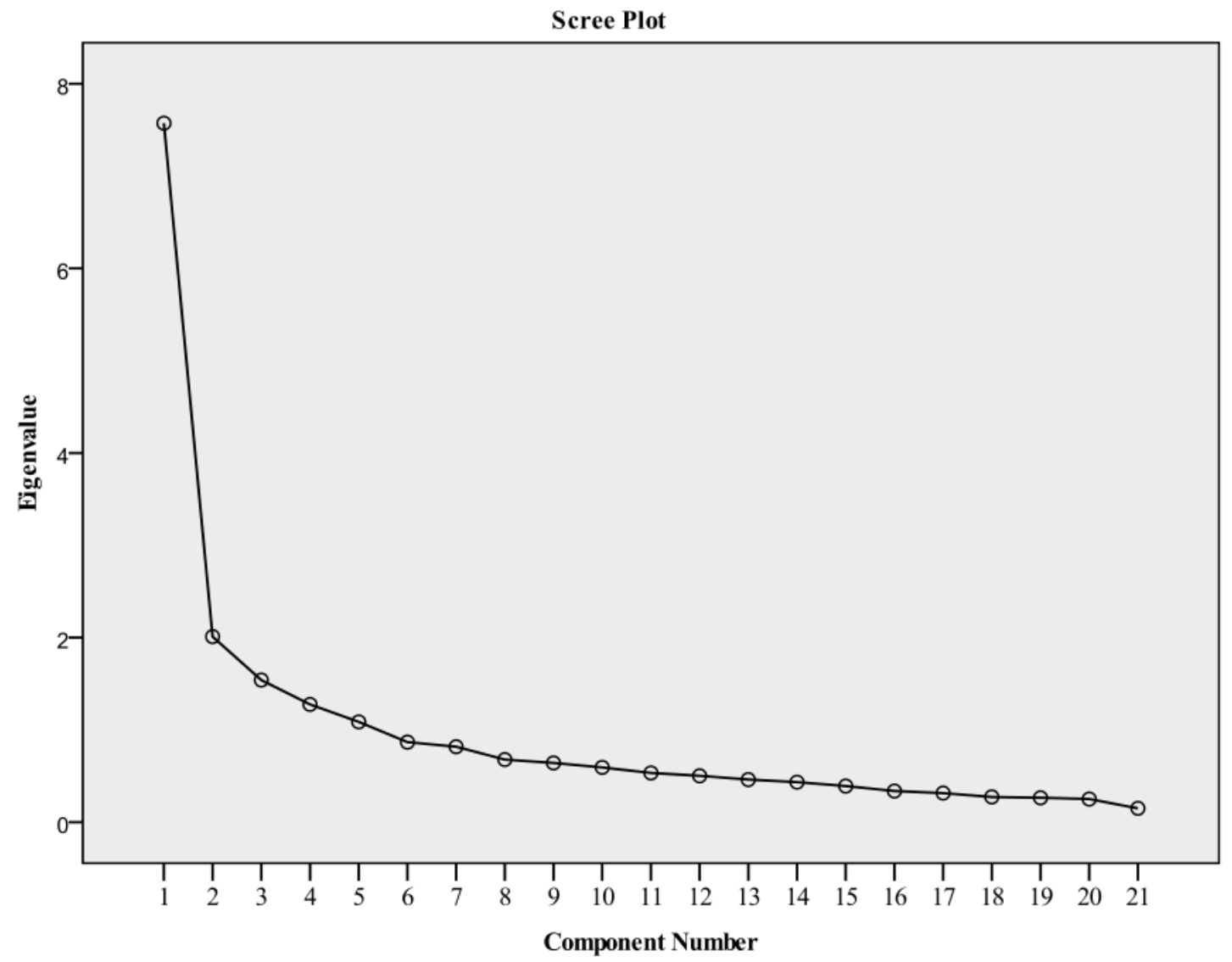

Figure 3. Results of scree plot from the 21-item SCAS-R.

Table 3

Results of Variance Explained from 21-item SCAS-R

\begin{tabular}{lcccccc}
\hline & \multicolumn{3}{c}{ Initial Eigenvalues } & \multicolumn{3}{c}{$\begin{array}{c}\text { Extraction Sums of Squared } \\
\text { Loadings }\end{array}$} \\
\cline { 2 - 7 } Component & Total & $\begin{array}{c}\text { \% of } \\
\text { Variance }\end{array}$ & $\begin{array}{c}\text { Cumulative } \\
\%\end{array}$ & Total & $\begin{array}{c}\% \text { of } \\
\text { Variance }\end{array}$ & $\begin{array}{c}\text { Cumulative } \\
\%\end{array}$ \\
\hline 1 & 7.57 & 36.06 & 36.06 & 7.57 & 36.06 & 36.06 \\
2 & 2.01 & 9.57 & 45.64 & 2.01 & 9.57 & 45.64 \\
3 & 1.54 & 7.33 & 52.97 & 1.54 & 7.33 & 52.97 \\
4 & 1.28 & 6.08 & 59.05 & 1.28 & 6.08 & 59.05 \\
5 & 1.09 & 5.18 & 64.22 & 1.09 & 5.18 & 64.22 \\
6 & .87 & 4.13 & 68.35 & & & \\
7 & .82 & 3.89 & 72.24 & & & \\
8 & .68 & 3.23 & 75.48 & & &
\end{tabular}




\begin{tabular}{llll}
9 & .64 & 3.06 & 78.53 \\
10 & .59 & 2.83 & 81.36 \\
11 & .53 & 2.54 & 83.90 \\
12 & .50 & 2.39 & 86.29 \\
13 & .46 & 2.20 & 88.49 \\
14 & .43 & 2.07 & 9.56 \\
15 & .39 & 1.86 & 92.42 \\
16 & .34 & 1.61 & 94.03 \\
17 & .32 & 1.50 & 95.53 \\
18 & .27 & 1.30 & 96.83 \\
19 & .27 & 1.26 & 98.09 \\
20 & .25 & 1.19 & 99.29 \\
21 & .15 & .71 & 10.00 \\
\hline
\end{tabular}

The factors were rotated using oblique Promax rotation. This rotation method was chosen because correlations among the factors were expected, and the use of oblique rotation creates a more reliable and accurate solution (Costello \& Osbourne, 2005). Items within the rotated pattern matrix loading onto the five retained factors were then analysed (Rummel, 1970). According to Tabachnick and Fidell (2001) and Costello and Osbourne (2005), no factor loadings should fall below .4 or cross-load with other factors at $\geq .32$. Item loadings on the first factor ranged from .66 to .84 and accounted for $36.06 \%$ of item variance (eigenvalue $=7.57$ ). The second factor's eigenvalue was 2.01 , held $9.57 \%$ variance, and item loadings ranged from .76 to .88 . The third factor accounted for $7.33 \%$ variance (eigenvalue $=$ 1.54 ) and item loadings were from .59 to .87 . The fourth factor held $6.08 \%$ of item variance and had an eigenvalue of 1.28. The lowest item loading for factor four was .40 and the highest was .89. The fifth and final factor had an eigenvalue of 1.09 and was found to account for $5.18 \%$ variance. Item loadings for the fifth factor were .89 and .96 . These factor loadings, as well as the 21 SCAS-R items, are presented in Table 4.

Table 4

SCAS-R Factor Loadings for Exploratory Factor Analysis with Oblique Promax Rotation

\begin{tabular}{|c|c|c|c|c|c|}
\hline SCAS-R Items & 1 & 2 & 3 & 4 & 5 \\
\hline Interacting at social events. & .86 & .00 & -.01 & -.05 & -.02 \\
\hline $\begin{array}{l}\text { Accurately interpreting and responding to other } \\
\text { people's gestures and facial expressions. }\end{array}$ & .81 & -.10 & -.02 & .06 & .00 \\
\hline
\end{tabular}


Varying the rate of my speaking in a culturally

.77 appropriate manner.

Interacting with members of the opposite sex.

Changing my behaviour to suit social norms, rules, attitudes, beliefs, and customs.

Building and maintaining relationships.

Accurately interpreting other people's emotions.

Managing my academic/work responsibilities.

Working effectively with other students/work colleagues.

Gaining feedback from other students/work

colleagues to help improve my performance.

Expressing my ideas to students/work colleagues in a culturally appropriate manner.

Maintaining my hobbies and interests.

Obtaining community services I require.

Attending or participating in community activities.

Dealing with the bureaucracy.

Adapting to the noise level in my neighbourhood.

Adapting to the population density.

Finding my way around.

Adapting to the pace of life.

Understanding and speaking the host language.

Reading and writing the host language.

\begin{tabular}{|c|c|c|c|c|}
\hline .77 & .05 & .00 & .15 & -.11 \\
\hline .73 & .02 & .15 & -.18 & .01 \\
\hline .72 & .07 & -.01 & .19 & -.15 \\
\hline .66 & .13 & -.07 & -.03 & .12 \\
\hline-.24 & .88 & .00 & .14 & -.02 \\
\hline .15 & .84 & -.01 & -.06 & -.06 \\
\hline .08 & .83 & -.08 & .01 & .03 \\
\hline .13 & .76 & .09 & -.15 & .05 \\
\hline-.26 & .09 & .87 & -.04 & -.01 \\
\hline .12 & -.06 & .76 & -.05 & .02 \\
\hline .15 & -.04 & .61 & .08 & .04 \\
\hline .24 & -.08 & .59 & .04 & -.08 \\
\hline-.03 & -.02 & -.13 & .89 & -.03 \\
\hline .10 & .04 & -.03 & .67 & .07 \\
\hline .03 & -.08 & .14 & .67 & .01 \\
\hline-.04 & .19 & .25 & .40 & .10 \\
\hline .06 & -.02 & -.03 & -.05 & .96 \\
\hline-.04 & .01 & .03 & .10 & .8 \\
\hline
\end{tabular}

Note. Factor loadings > .40 are in boldface. $1=$ Interpersonal Communication; $2=$ Academic/Work Performance; 3 = Personal Interests \& Community Involvement; 4 = Ecological Adaptation; 5 = Language Proficiency.

The results of the EFA pattern matrix largely supported the study's hypotheses. The first SCAS-R factor relates to items involving communication and social interaction (e.g., interacting at social events, accurately interpreting other people's emotions), and was labelled Interpersonal Communication. The second factor has been labelled Academic/Work Performance due to the loading of items such as "Managing my academic/work responsibilities" and "Expressing my ideas in class/at work in a culturally appropriate manner." The third factor included items involving community engagement and cultural maintenance (e.g., "Maintaining my hobbies and interests", "Attending or participating in 
community activities"), and was labelled Personal Interests \& Community Involvement. As items that loaded together such as "adapting to the population density" and "adapting to the noise level in my neighbourhood" involved ecology and the environment, the forth factor was labelled Ecological Adaptation. The fifth and final factor was termed Language Proficiency, as items centred around host language fluency (e.g., "Understanding and speaking the host language" and "Reading and writing the host language").

With the exception of the fifth Language Proficiency factor, the other four factors corroborated the initial conceptualisation of the SCAS-R as having four distinct but interrelated domains. The two items involving language proficiency were initially placed under the Interpersonal Communication factor given the importance of language in communicating with and understanding others. However, as both of the language items included in the SCAS-R were found to load onto one factor, were correlated highly with one another $(r=.82, p<.01)$, and had extremely low cross-loadings with other factors, the decision to retain the fifth factor was upheld ${ }^{1}$.

The final step of the EFA examined the overall internal consistency of the SCAS-R as well as the five factor or subscale alphas (Cronbach, 1951). Results indicated acceptable internal reliability for both the subscales and overall SCAS-R (see Table 5). Outside of one marginally acceptable item correlation ("Adapting to the noise level in my neighbourhood"; $r$ $=.29$ ), all subscale inter-item correlations were above the cut-off of .30 (Stevens, 1992). Correlations between the sub-scales are presented in Table 7.

Table 5

Internal Consistency, Means, and Standard Deviations of the SCAS-R and SCAS-R Subscales

\begin{tabular}{lccc}
\hline SCAS-R Subscales & $\alpha$ & M & SD \\
\hline Interpersonal Communication & .91 & 3.64 & .78 \\
Academic/Work Performance & .86 & 3.90 & .86 \\
Personal Interests \& Community & & & \\
Involvement & .76 & 3.23 & .85 \\
Ecological Adaptation & .71 & 3.91 & .74 \\
Language Proficiency & .90 & 3.95 & 1.15 \\
Overall SCAS-R & .92 & 3.75 & .62 \\
\hline
\end{tabular}

${ }^{1}$ A factor with two items may be considered sufficient, although this is generally seen as an exception rather than a norm (Bollen, 1989; Marsh, Hau, Balla, \& Grayson, 1998; Raubenheimer, 2004); more items per factor engenders replicability (Little, Lindenberger, \& Nesselroade, 1999; Velicer \& Fava, 1998). 


\section{Construct Validity}

A number of one-tailed correlations were executed to examine the internal validity of the SCAS-R against other convergent measures of sociocultural adaptation, psychological adjustment, and social/behavioural skills. The following table (Table 6) illustrates confirmation of the hypotheses regarding the anticipated relationship between these various scales. In particular, the SCAS-R was found to have a strong, negative relationship with the original SCAS, a large and positive correlation with the SAS, a positive correlation with the SWLS, and a negative relationship with the ZSDS. Further, the SCAS-R correlated positively and strongly with the SSI, the CQB, and the SAB.

It was also of interest to examine the correlational relationships between the original SCAS, the revised SCAS-R, and the criterion measures. Overall, the results show similar patterns between the original and revised SCAS and other included measures with some exceptions. Contrary to hypotheses, however, the SCAS-R exhibited larger overall relationships with the two psychological adjustment measures than anticipated, correlating with the SWLS and ZSDS at $r=.51$ and $r=-.49$, respectively. These correlations were both larger than the $r=.42$ correlation found between the SCAS and psychological adjustment in Study 1. However, in line with hypotheses, correlations between the SCAS-R and behavioural and social skill measures (e.g., the CQB, SAB, and SSI) were generally greater than the associations found between the SCAS and these instruments.

A number of one-tailed correlations were also conducted to test specific hypotheses relating to the SCAS-R subscales and other included measures. The SCAS-R Interpersonal Communication (IP) subscale, concordant with hypotheses, was correlated with the SAS Interaction Adjustment subscale $(r=.60, p<.001)$, SSI $(r=.47, p<.001)$, and CQB $(r=$ $.33, p<.001$ ). Further, the Academic/Work Performance (AWP) subscale was found to have a large, positive relationship with the SAS Work Adjustment subscale $(r=.56, p<.001$, respectively). In line with predictions, the Personal Interests and Community Involvement (PICI) subscale was moderately correlated with the SAB $(r=.43, p<.001)$. As was previously mentioned, a complete table with all subscale correlations is presented in Table 7 . 
Table 6

Correlations Between SCAS-R and Other Adjustment Indices

\begin{tabular}{|c|c|c|c|c|c|c|c|c|}
\hline Measure & 1. & 2. & 3. & 4. & 5. & 6. & 7. & 8. \\
\hline $\begin{array}{l}\text { 1. SCAS-R } \\
(.93)\end{array}$ & - & -.60 & .67 & .51 & -.49 & .37 & .53 & .35 \\
\hline $\begin{array}{l}\text { 2. SCAS } \\
(.92)\end{array}$ & & - & -.61 & -.40 & .53 & -.19 & -.53 & -.29 \\
\hline $\begin{array}{l}\text { 3. SAS } \\
(.93)\end{array}$ & & & - & .52 & -.38 & .28 & .61 & .26 \\
\hline $\begin{array}{l}\text { 4. SWLS } \\
(.87)\end{array}$ & & & & - & -.51 & .13 & .47 & .30 \\
\hline $\begin{array}{l}\text { 5. } \begin{array}{l}\text { ZSDS } \\
(.81)\end{array}\end{array}$ & & & & & - & -.13 & -.31 & -.20 \\
\hline $\begin{array}{l}\text { 6. } \mathrm{CQB} \\
(.90)\end{array}$ & & & & & & - & .23 & .33 \\
\hline $\begin{array}{r}\text { 7. SAB } \\
(.80)\end{array}$ & & & & & & & - & .36 \\
\hline $\begin{array}{l}\text { 8. SSI } \\
(.81)\end{array}$ & & & & & & & & - \\
\hline
\end{tabular}

Note. Cronbach's alphas are displayed in parentheses. Correlations between SCAS-R and other scales are in boldface. SCAS-R = Revised Sociocultural Adaptation Scale; SCAS = Original Sociocultural Adaptation Scale; SAS = Subjective Adjustment Scale; SWLS = Satisfaction With Life Scale; ZSDS = Zung Self-Rating Depression Scale; CQB = Cultural Intelligence Behaviour Component; $\mathrm{SAB}=$ Sociocultural Adaptation Behaviour in Host Country; SSI = Social Skills Inventory.

All correlations are significant at the .01 level (one-tailed). 
Table 7

Correlations Between SCAS-R Subscales and Other Adjustment Indices

\begin{tabular}{|c|c|c|c|c|c|c|c|c|c|c|c|c|c|}
\hline Measure & 1. & 2. & 3. & 4. & 5. & 6. & 7. & 8. & 9. & 10. & 11. & 12. & 13. \\
\hline 1. IP & - & .55 & .59 & .49 & .42 & .60 & .33 & .55 & .43 & -.39 & .33 & .53 & .47 \\
\hline 2. AWP & & - & .38 & .38 & .24 & .40 & .56 & .42 & .37 & -.40 & .27 & .31 & .32 \\
\hline 3. PICI & & & - & .47 & .40 & .47 & .30 & .51 & .40 & -.38 & .18 & .43 & .24 \\
\hline 4. EA & & & & - & .48 & .38 & .29 & .44 & .32 & -.32 & .26 & .32 & .23 \\
\hline 5. LP & & & & & - & .40 & .23 & .35 & .17 & -.23 & $.04^{\sim}$ & .39 & $.06^{\sim}$ \\
\hline 6. IA & & & & & & - & .46 & .86 & .45 & -.32 & .24 & .66 & .34 \\
\hline 7. WA & & & & & & & - & .63 & .29 & -.22 & .15 & .31 & .20 \\
\hline 8. GA & & & & & & & & - & .51 & -.39 & .27 & .51 & .16 \\
\hline 9. SWLS & & & & & & & & & - & -.51 & .13 & .47 & .30 \\
\hline 10. ZSDS & & & & & & & & & & - & -.13 & -.31 & -.20 \\
\hline 11. CQB & & & & & & & & & & & - & .23 & .33 \\
\hline 12. SAB & & & & & & & & & & & & - & .36 \\
\hline 13. SSI & & & & & & & & & & & & & - \\
\hline
\end{tabular}

Note. IP = SCAS-R Interpersonal Communication; AWP = SCAS-R Academic/Work Performance; PICI = SCAS-R Personal Interests and Community Involvement; EA = SCAS-R Ecological Adaptation; LP= SCAS-R Language Proficiency; IA = SAS Interaction Adjustment; WA = SAS Work Adjustment; GA = SAS General Adjustment; SWLS = Satisfaction With Life Scale; ZSDS = Zung Self-Rating Depression Scale; CQB = Cultural Intelligence Behaviour Component; SAB = Sociocultural Adaptation Behaviour in Host Country; SSI = Social Skills Inventory.

All correlations are significant at the .01 level (one-tailed) except ${ }^{\sim}, p>.01$. 
Notable differences between some of the SCAS-R subscales and psychological adjustment (life satisfaction and depression) were also discovered. Individuals who reported having greater adaptation competency on the IP subscale also reported higher scores on the SWLS at $r=.43, p<.001$, which was a slightly larger correlation than was found between the IP and ZSDS, $r=-.39, p<.001$. Whereas interpersonal communication competency appeared to relate more with life satisfaction than depression, the LP subscale, conversely, was found to have the opposite relational pattern. Higher language proficiency scores corresponded to a stronger relationship with depression $(r=-.23, p<.001)$ than life satisfaction, $r=.17, p<.001$.

\section{Discussion}

Three main concerns with the existing SCAS were addressed in the present study through the development and examination of a revised measure of sociocultural adaptation. First, development of the revised measure sought to decrease the conceptual overlap with psychological adjustment and more solidly position the SCAS-R within the culture learning framework as a self-report measure of behavioural competency through removal of potentially emotive or affective terminology within the SCAS. In a second but related issue, terminology in the SCAS-R was utilised to highlight behavioural adjustment, an improvement over the current SCAS's emphasis on adaptation difficulty or maladjustment to a new culture. Third, although some efforts have been previously made to measure multiple dimensions of behavioural competency in cross-cultural situations (Black, Gregersen, \& Mendenhall, 1992; Black, Mendenhall, \& Oddou, 1991; Black \& Stephens, 1989; Brein \& David, 1971; Feldman, 1976; Hawes \& Kealey, 1981), this study represents a preliminary attempt to develop a multi-domain measure of sociocultural adaptation that is situated within the culture learning framework of acculturation psychology.

\section{Exploratory Factor Analysis, Internal Reliability, Construct Validity of the SCAS-R, and General Findings}

Development of the SCAS-R included examination of the instrument's factor structure, as well as its internal reliability and construct validity. First, results largely corroborate the initial conceptualisation of the SCAS-R as having four distinct but interrelated domains (Interpersonal Communication, Personal Interests and Community Involvement, Academic/Work Performance, and Ecological Adaptation) with the exception of an unanticipated fifth Language Proficiency factor. 
Although these two items involving language ability were not hypothesised to create a subscale on their own, conceptual retention of the SCAS-R language subscale is reinforced by previous literature concerning the significance of host language competency. This is most notably evidenced in Masgoret and Ward's (2006) representation of foreign language ability as a central component of effective cross-cultural interaction and sociocultural adaptation. Other researchers (Church, 1982; Clément \& Bourhis, 1996; Kang, 2006; Noels, Pon, \& Clément, 1995; Ward, 2004; Ward \& Kennedy, 1993a) have also found that foreign language competence, as well as self-confidence in speaking a host country's language, are vital prerequisites in building meaningful interpersonal relationships and social support networks with members of the host society, for the completion of day-to-day tasks, and for successful cross-cultural adjustment.

Further methodological support for retention of the Language Proficiency domain is based on previous psychometric research suggesting that scales with multiple factors may have as little as two items per factor, although the usual case is a minimum of three items in order to improve chances of replication (Bollen, 1989; Little, Lindenberger \& Nesselroade, 1999; Marsh, Hau, Balla, \& Grayson, 1998; Raubenheimer, 2004; Velicer \& Fava, 1998). Anderson and Gerbing (1984) provide further validation for inclusion of the two-item subscale. They suggest that a sample size of 150, which is well below the current study's 316 participants, is usually sufficient for two indicators in a factor to be convergent and have a proper solution, and that high item loadings of approximately .9 per item can provide good convergence for two-item factors (factor loadings for the language subscale were .96 and .89). Ultimately, inclusion of a language proficiency domain within the SCAS-R and the domain's central positioning within the sociocultural adaptation construct is a relatively novel theoretical approach that, although validated both conceptually and methodologically here, deserves continued psychometric attention in future acculturation research.

Results also indicate acceptable internal reliability for the five SCAS-R subscales as well as the SCAS-R as an overall whole. Alphas span from .92 (overall SCAS-R) to .71 (the Ecological Adaptation subscale). Although all subscale inter-item correlations are above the acceptable cut-off point of .30 (Stevens, 1992) and all alphas are above .70-a threshold considered adequate for psychometric measurements due to the diversity of constructs measured within the field (Kline, 1999) - future research investigating the lower Ecological Adaptation subscale alpha of .71 is warranted in order to provide supplementary evidence of the reliability, consistency, and sufficient power of this domain. 
Construct validity is also evidenced in the SCAS-R and its various domains. Specifically, hypotheses regarding the construct validity of the SCAS-R are confirmed through significant correlations with other measures of adaptation, behaviour, and social skills (e.g., the SCAS and the CQB). Each of these criterion instruments relate to the appropriateness of either verbal and/or physical actions within different intra- and intercultural situations, therefore the congruent correlational evidence found suggests that the SCAS-R adequately reflects the learning and behavioural competency themes attributed to the sociocultural adaptation construct (Bochner, 1981; Furnham \& Bochner, 1986; Ward, Bochner, \& Furnham, 2001; Ward \& Kennedy, 1999).

Evidence of the construct validity of various sociocultural adaptation domains is further demonstrated through significant subscale correlations between the SCAS-R and other included criterion measures and their subscales. For example, an importance facet of sociocultural adaptation related to interpersonal communication and effective social interactions is reflected in the significant association between the SCAS-R Interpersonal Communication and SAS Interaction Adjustment subscales. Furthermore, the medium-sized correlations between the Academic/Work Performance and SAS Work Adjustment subscales provide support for the premise that behavioural skill acquisition and adaptation occurs within a contextual (e.g., work or academic) performance environment (Berno \& Ward, 2003; Black \& Stephens, 1989; Pulakos et al., 2003; Ward \& Masgoret, 2004), and the SCAS-R Personal Interests and Community Involvement and participants' host country behaviours subscale (SAB) correlation highlights the importance of maintaining one's personal interests and involving one's self in the host society (Ishiyama \& Westwood, 1992; Massimini \& Delle Fave, 2000; Stahl \& Caligiuri, 2005).

Last, a central objective of the study was to phrase the SCAS-R in a way that would capture behavioural rather than emotive responses to cross-cultural transition. These behavioural responses were hoped to engender a more unequivocal repositioning of the sociocultural adaptation construct within the framework of culture learning by further separating it from the stress and coping paradigm. To test this premise, two criterion indices of psychological adjustment were included - the SWLS and the ZSDS - and were expected to issue medium-sized correlations with the SCAS-R. In contrast to hypotheses, however, correlations between the SCAS-R, life satisfaction, and depression are somewhat larger than anticipated ( $r=.51$ and -.49 , respectively). However, the SCAS-R also demonstrates an overall stronger association to the behavioural criterion measures than the SCAS - the CQB 
in particular-which supports the revised measure's more solid repositioning within the culture learning domain.

\section{Limitations and Future Directions}

Although these initial findings are encouraging, there are a number of limitations that merit comment. One limitation is the unavailability of additional reliability and validity studies. Although this shortcoming will be partially addressed in the following chapter, various research constraints in the present study made more thorough development of a psychometric research programme unachievable (e.g., convenience sampling); continued work in this regard will be necessary in order to publish the SCAS-R. Second, the study does not examine the discriminant validity of the SCAS-R: Despite evidence of convergent validity, the extent to which the scale measures sociocultural adaptation-as opposed to tapping into broader or similar constructs-has not been thoroughly investigated. Again, further validation work would increase confidence in the measure.

Another limitation concerns shortcomings with the construction and validation of self-report measures and, in this instance, self-report measures of competency. Behavioural self-report measures are inherently subjective in nature, as they are based on an individual's assessment of their behaviour that may or may not adequately reflect the more objective nature of their skills. Given the debate surrounding self-report measures (see Howard, Maxwell, Wiener, Boynton, \& Rooney, 1980; Podsakoff, MacKenzie, Lee, \& Podsakoff, 2003; Stone et al., 2000) a multi-method approach would offer a way to further substantiate self-report findings. Such approaches include longitudinal or experimental designs, as well as performance assessments or independent observations of behaviour and reports from others (e.g., co-workers, friends, relatives). Multi-method approaches included in future research regarding psychometric development of the SCAS-R would provide additional support for the scale's validity.

Various issues with the sample also posed some limitations to the findings. Translation of the SCAS-R into multiple languages was nonviable due to research constraints, leaving the question of cross-cultural equivalence unanswered. Further, as the SCAS-R was provided in English only, concurrent validity is limited to participants who either spoke English as a first language or reported having high levels of English language proficiency. Whether similar results would be obtained in samples with lower host language fluency remains unknown. Another limitation concerns the fact that the study does not examine cross-cultural differences in SCAS-R scores: The extent to which the SCAS-R, as 
well as its antecedents and consequences, is moderated by cultural context is an avenue of cross-cultural comparative research worth exploring. Various cross-cultural research methodologies such as direct comparison, a combined "etic-emic" approach, and item response (Hui \& Triandis, 1985) could be utilised in this regard. Overall, cross-validation work with a sample of participants with varying degrees of host language ability and/or backtranslated versions of the SCAS-R in multiple languages would be extremely valuable and again necessary for publication of the SCAS-R.

A final methodological point concerns various issues with exploratory factor analysis (EFA). As Tabachnick and Fidell (2007) relate, EFAs by nature do not have existent or external criteria available for solution testing: An endless number of rotations and factors to retain are present, which leaves a degree of ambiguity requiring interpretation and decisions that may very well vary between researchers. Further, different participant samples will also likely result in variant EFA results. An awareness of these issues paired with further validation research would go some way in addressing these potential methodological concerns.

In sum, the present study represents the initial developmental stages of a revised measure of sociocultural adaptation. It is hoped that the target concept has been clearly conceptualised and the analyses have been conducted with as much theoretical and empirical clarity as possible in order to allow for later stages of measurement validation and refinement to proceed. Further, the SCAS-R represents a novel contribution to acculturation literature and related fields with particular regard to the 5-factor scale approach taken here. Further investigation of the empirical evidence presented in this chapter regarding multiple behavioural adaptation domains within the acculturation framework would be beneficial in terms of delineating the relationships that exist between various antecedents and specific adjustment domains, providing more contextual information about what sociocultural areas of adjustment individuals find more challenging than others, and adding a more comprehensive understanding of how behavioural competency changes dependent upon differing domains individuals experience in a host society. Ultimately, division of cross-cultural behavioural competencies into these separate domains can provide a foundation for more in-depth exploration of sociocultural adaptation and cultural transition more generally.

The SCAS-R, like its predecessor, may be utilised in research concerning acculturating persons, particularly migrants, international students, and expatriates. It complements existing measures of social skills and psychological adjustment, which can provide a valuable tool for exploring cross-cultural adaptation in multicultural contexts. 
Further, although the SCAS-R is designed primarily as a research tool-as a means of assessing individual differences in cross-cultural behavioural competency and for examining skill acquisition and adjustment in novel cultural contexts-it also has practical applications in international management, business, and educational settings. The SCAS-R places emphasis on behavioural skill acquisition, and skill development inherently involves a learning process. Therefore, it seems reasonable to assume that people can develop and enhance these basic skills. Practice and training with these skills should lead to learning more effective intercultural behaviours, and as such the SCAS-R may be a good starting point for initial or follow-up training assessments.

The following chapter details the third and final study of the research programme on sociocultural adaptation. In light of the findings and limitations of both the meta-analysis and the current exploratory study, subsequent research objectives will continue to extend the boundaries of sociocultural adaptation research through: (1) Corroboration of the five-factor structure of the SCAS-R using confirmatory factor analysis; (2) continued investigation into the validity of the SCAS-R as a behavioural facet of cultural competency as compared to other affective adjustment criterion measures; (3) examination of the motivational and discrimination influences on sociocultural adaptation, and (4) investigation of sociocultural adaptation as an antecedent to psychological adjustment. 


\section{Chapter Four: Migration Motivation, Cultural Competence, and Discrimination: Potential Pathways to Psychological Adjustment}

Selected findings from the SCAS-R validation study (Chapter 3) and meta-analysis (Chapter 2) are further considered in this final component of the sociocultural adaptation research programme. The previous chapter presented a revised measure of sociocultural adaptation competency, the SCAS-R, and its five subscales: Interpersonal Communication (IC), Personal Interests and Community Involvement (PICI), Academic/Work Performance (AWP), Ecological Adaptation (EA), and Language Proficiency (LP). The following chapter continues investigation of the psychometric and conceptual underpinnings of the SCAS-R. In particular, corroboration of the revised measure's five-factor structure and internal consistency of the scale using a new participant sample will be sought.

The second objective of the present study arises from meta-analytic results in Chapter 2, which found strong associations between sociocultural adaptation and two underrepresented variables in the acculturation literature, motivation and perceived discrimination. For example, one facet of motivation included in the meta-analysis, crosscultural self-efficacy, was found to have one of the strongest effect sizes $(r=.47)$ of all the meta-analytic variables, including more traditional culture learning constructs where correlations ranged from $r=.14$ (co-national contact) to $r=.38$ (language ability). Further, the discrimination-adaptation relationship was the second strongest meta-analysis correlate $(r$ $=-.54)$, though this effect size was comprised of only 6 studies. Because of the relatively small amount of research regarding motivation, discrimination, and cross-cultural adjustment outcomes, the following study aims to make further contributions to the acculturation literature through exploration of these variables in relation to psychological adjustment and behavioural competency as measured by the SCAS-R.

A novel approach to sociocultural adaptation is presented through empirical examination of two hypothetical path models integrating the aforementioned constructs of motivation and perceived discrimination. In particular, these path models test direct and indirect pathways between reasons or factors for moving abroad (preservation, familial, and lifestyle motives; to be discussed in further detail subsequently), migration motivation (conceptualised by two different facets of cross-cultural motivation; autonomous regulation and Motivational CQ), cross-cultural behavioural competency, discrimination, and psychological adjustment. 
These models are an innovative approach within the acculturation literature in two key ways. First, as will be outlined in more detail presently, previous research that exists on the topic has focussed almost exclusively on the independent associations between motivation, discrimination, and cross-cultural adjustment. Such studies have involved the influence of motivation on performance (e.g., Baard, Deci, \& Ryan, 2004), or how perceptions of discrimination affect the health and psychological well-being of ethnic minorities and migrant populations (e.g., Lee \& Ahn, 2012). Only a modest amount of literature has considered the possible linkages among these various constructs, such as how greater degrees of behavioural competencies in novel cultural environments may be predictive of less perceived discrimination and in turn better psychological outcomes (Dalhaug, Oppedal, \& Røysamb, 2011; Jasinskaja-Lahti, 2008; Jasinskaja-Lahti \& Liebkind, 2007; Neto, 2006). A further innovation concerns the examination of double-mediation pathways, whereby both sociocultural adaptation competency and perceived discrimination are hypothesised to mediate the relationship between aspects of motivation and psychological well-being. There are no known acculturation studies to date investigating this hypothesised mediational pathway. As such, the two hypothesised models represent a novel research endeavour due to the direction of hypothesised effects and the concurrent consideration of the linkages between these variables.

The first hypothesised path model considers the potential effects of sociocultural adaptation competency and perceived discrimination on the association between migrants' reasons for moving abroad and their psychological well-being. Specifically, it is posited that migrants who moved to New Zealand due to lifestyle reasons (e.g., more relaxed pace of life, outdoor opportunities) will report positive psychological outcomes, whereas migrants who relocated to New Zealand because of preservation factors (e.g., safety from crime) will report poorer psychological well-being. Further, it is suggested that adaptation competency and experiences of discrimination will mediate the relationship between these migration factors and psychological adjustment (Figure 4). 


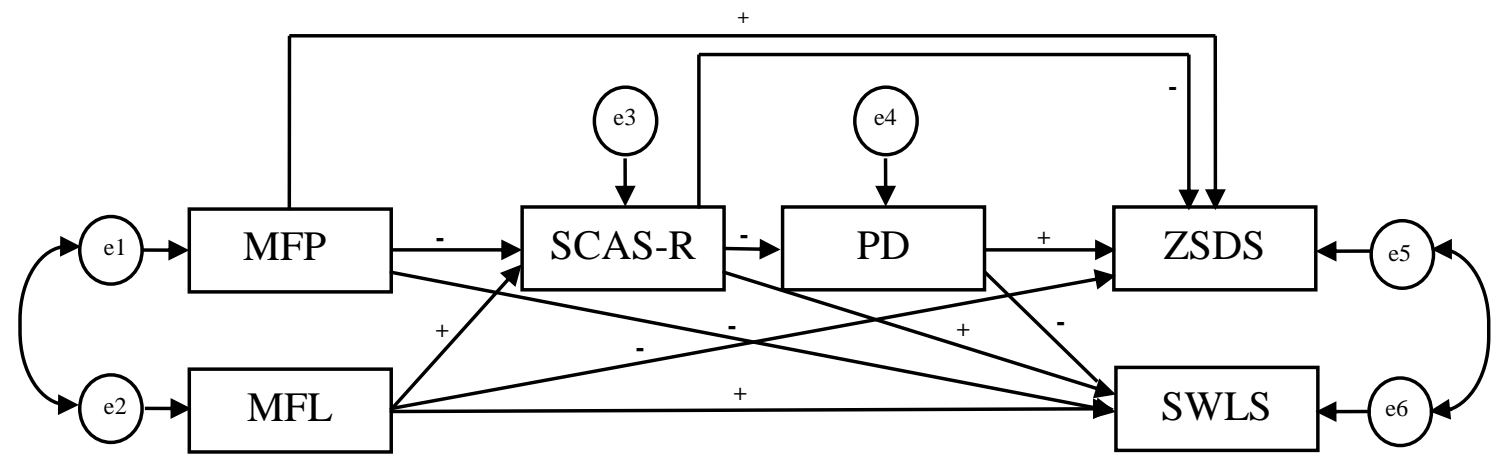

Figure 4. Hypothesised path model illustrating the mediating effects of sociocultural adaptation and perceived discrimination on the relationship between migration factors and psychological adjustment outcomes.

Note. $\quad$ MFP $=$ preservation migration factors; MFL = lifestyle migration factors; SCAS-R = sociocultural adaptation; $\mathrm{PD}=$ perceived discrimination; ZSDS = depression; SWLS = life satisfaction.

The second integrated path model investigates the potential pathways between two forms of migration motivation-Motivational $\mathrm{CQ}$ and autonomous regulation-and behavioural competency, discrimination, and psychological well-being. It is suggested that migrants with greater levels of migration motivation will report positive mental health outcomes, but that this association will be mediated by both a migrant's sociocultural adaptation competency and his or her perceptions of discrimination. More specifically, the second path model puts forth the premise that migrants who are incentivised to learn and enact culturally relevant skills in a new culture may perceive or experience less discrimination towards them, which then leads to more life satisfaction and less depression. A hypothetical path model was created (Figure 5) in order to describe the potential nature of these relationships.

To summarise, the current study will address theoretical gaps in the culture contact literature and extend the boundaries of the sociocultural adaptation construct through (1) confirmation of the SCAS-R's factor structure and reliability of the instrument, and (2) investigation of potentially causal pathways between various facets of motivation, sociocultural adaptation competency, discrimination, and psychological well-being. 


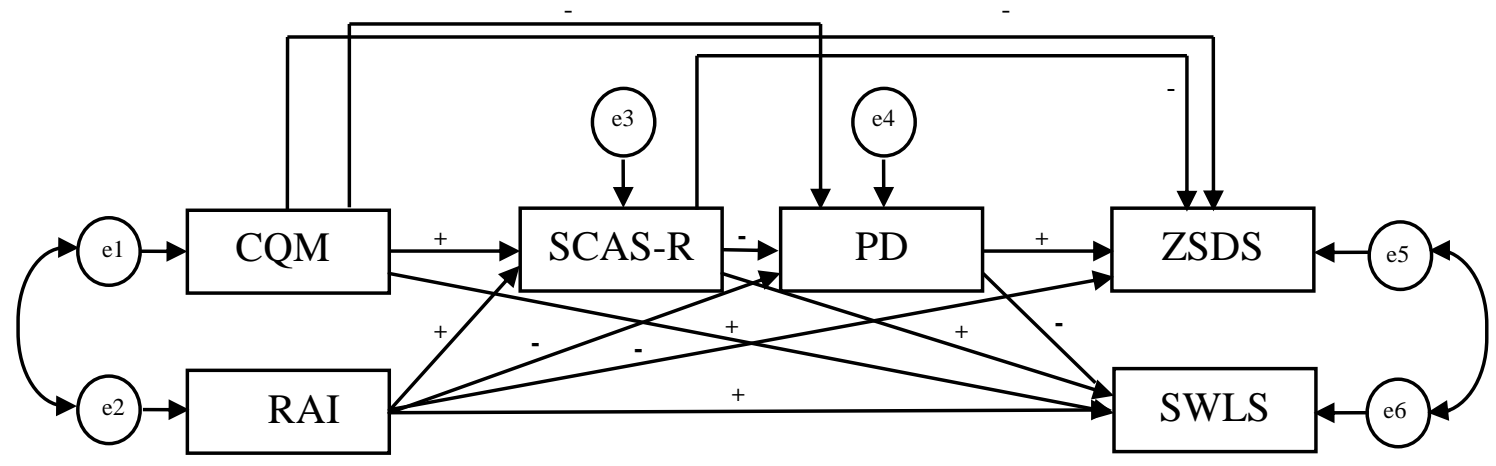

Figure 5. Hypothesised path model illustrating the mediating effects of sociocultural adaptation and perceived discrimination on the relationship between migration motivation and psychological adjustment outcomes.

Note. $\mathrm{CQM}=$ Motivational $\mathrm{CQ} ; \mathrm{RAI}=$ autonomous regulation; $\mathrm{SCAS}-\mathrm{R}=$ sociocultural adaptation; $\mathrm{PD}=$ perceived discrimination; ZSDS = depression; SWLS $=$ life satisfaction.

Further commentary on the aforementioned research objectives is presented next, beginning with a brief synopsis of exploratory versus confirmatory factor analyses. A literature review is then examined in six sections. First, theoretical and empirical work concerning reasons for migration, migration motivation, and cross-cultural transition-as conceptualised both by psychological adjustment and sociocultural adaptation-is reviewed. The relationships between sojourning individuals' perceived discrimination and intercultural adjustment is considered, followed by an overview of research on the motivationdiscrimination association. Fourth, an empirically based argument is outlined with regard to how sociocultural adaptation, as a behavioural measure of cultural competence, may be considered as an antecedent to psychological adjustment. Fifth, both sociocultural adaptation and perceived discrimination are considered as potential mediators of the relationship between motivation and psychological well-being. Last, a summary of the hypothesised causal pathways between these aforementioned constructs is provided along with delineation of the study's hypotheses.

\section{Psychometric Properties of the SCAS-R}

Using exploratory factor analysis (EFA), the previous chapter presented the development of a revised sociocultural adaptation measure with five subscales or factors grounded within various theoretical frameworks (e.g., culture learning, cultural intelligence). The present study seeks to corroborate this factor structure using confirmatory factor analysis or CFA. Whereas EFA is used to identify a factor structure, CFA is a priori in nature as it confirms the factors produced from the EFA and allows for specific hypotheses about the 
structure to be made (Fabrigar, Wegener, MacCallum, \& Strahan, 1999). In addition, the psychometric underpinnings of the SCAS-R are further examined for internal consistency. Cronbach's alphas for each of the factors and the overall SCAS-R will be investigated for acceptable reliability.

\section{Motivation and Adjustment}

Motivation is not a widely researched topic in the acculturation literature (Gezentsvey \& Ward, 2008). Padilla and Perez (2003) even suggest that psychological models of acculturation are "of limited value" as they fail to consider newcomers' motivations to acculturate (p. 50). Recommendations from other acculturation researchers have also been made for further investigation of how motivation and motivational factors relate to engagement and participation in host societies (e.g., Kosic, 2004; Winchie \& Carment, 1989). In further support of the need for additional research on motivation and acculturative processes, meta-analytic results from Chapter 2 revealed significant associations between migration motivation (e.g., reasons for international moves, integrative motivation) and cultural competency as measured by the SCAS, though fewer than 25 studies examining these variables were located. With this in mind, this section explores literature involving migrants' motives for moving abroad (e.g., migration factors), two specific migration motivation constructs-Motivational CQ and autonomous regulation-and the relationships between these components of motivation and cross-cultural adjustment.

Migration factors and adjustment outcomes. A rich history of multi-disciplinary research exists on factors that influence an individual's decision to migrate. In the late $19^{\text {th }}$ century German-English geographer and statistician Ernst Ravenstein was the first to publish information on migratory push-pull processes and the "laws of migration" using census data (Dorigo \& Tobler, 1983). Demographer Everett Lee furthered Ravenstein's work on pushpull processes through delineation of four main migration factors related to: (1) A migrant's country of origin; (2) the migrant's destination country; (3) geographical, physical, and economic barriers, and (4) personal factors (1966). The disciplines of geography, sociology, economics, and psychology alike have continued to examine push-pull determinants of migration (Chirkov, Vansteenkiste, Tao, \& Lynch, 2007; Martin, 1993). Push factors, for example, can include forced or reactive migration related to negative or unsatisfactory situations in an individual's country of origin that influence immigration, such as religious or political persecution or war (Berry, 1997; Marsella \& Ring, 2003; Richmond, 1993). Pull factors, conversely, are more proactive in nature, and include positive attributes an individual 
perceives in a host country that may potentially drive voluntary migration such as lifestyle or personal growth (Berry, 1997; Martin, 1993).

These push-pull migration factors may be further categorised according to differing kinds of migration goals. For example, Tartakovsky and Schwartz (2001) have termed one aspect of migration factors preservation motives, which they describe as reasons for relocation concerning physical, psychological, and social security. Another domain of migration factors may also involve familial relationships including filial duty (Cowling, 2002), relocation resulting from a partner's international job assignment (Tabor \& Milfont, 2012), or inter-generational migration of families abroad (Booth, Crouter, \& Landale, 1997). A third group of migration factors includes lifestyle motives, with specific migration determinants such as a country's standard of living, the ecology, pace of life, or the general atmosphere of a particular country (Benson \& O'Reilly, 2009; Lundmark, 2006; Winchie \& Carment, 1989) belonging to this category.

In the cross-cultural psychology literature, these push-pull determinants have been investigated in relation to various adjustment outcomes (Funham \& Bochner, 1986; Richmond, 1993). In relation to preservation and lifestyle factors, for example, Berry, Kim, Minde, and Mok (1987) found that both push (e.g., preservation) and pull (e.g., lifestyle) factors were positively related to acculturative stress. They surmised that those migrants forced to leave their home country may have experienced feelings of resentment and therefore higher levels of stress, and that people with pull motives for moving abroad may have had unrealistically high expectations which might have also resulted in higher acculturative stress. Similarly, Chirkov and colleagues (2007) and Tartakovsky and Schwartz (2001) found that preservation motives had strong negative associations with sociocultural and psychological adjustment outcomes such as positive affect and life satisfaction. Further, because the majority of preservation factors such as conflict-, disaster-, and developmentinduced displacement may be considered under the classification of forced or involuntary migration (FMO, 2012), a vast literature has been established examining the effects of these types of preservation migration factors and negative health and well-being indicators (for a review see Fazel, Wheeler, \& Danesh, 2005).

Family migration factors have also been associated with both positive and negative adaptation outcomes. For example, "trailing" or "tied" spouses (e.g., spouses of global professionals) appear to experience more adaptation and employment difficulties in a host society than their partners, who may be more motivated or enthusiastic to move due to the employment opportunities relocation presents (Cooke, 2001; Tabor \& Milfont, 2012). 
Conversely, partners of global professionals who felt they had some control over the migration process and were able to contribute to decisions related to the move have reported greater levels of satisfaction in the new host society (Hiller \& McCaig, 2007), and yet other research has not detected pre- versus post-migration differences in trailing spouses' life satisfaction (Nowok et al., 2011).

The voluntary nature of a migrant's international relocation for lifestyle purposes generally elicits positive adjustment outcomes. For example, one study found that a sample of retired Japanese expatriates living in Malaysia reported high levels of satisfaction with the quality of their retirement life (Ono, 2008). Moving abroad for lifestyle reasons has also been found to relate to the adoption of new cultural practices and generally positive experiences (Legido-Quigley \& McKee, 2012). Some participants (English retirees living in Spain) in the aforementioned qualitative research said, for example, that their "quest for "the good life" " had led to them feeling healthier, doing more exercise, and involving themselves in more social activities (2012).

When these previous research findings are considered in relation to the present study, it is believed that migrants who chose to move to New Zealand for lifestyle reasons will experience higher sociocultural adaptation, higher life satisfaction, and less depression, and that the opposite relationships will be evidenced for those individuals who moved due to preservation factors. Given the inconclusive findings on the relationship between familial factors and cross-cultural adjustment, no specific direct paths are hypothesised for this association.

In sum, the reasons why migrants leave their home countries due to reasons that range across a broad continuum of push factors (e.g., war or violence) and pull factors (e.g., to receive a better education abroad), and these factors in turn have been associated with the degree of adjustment they experience within their new host societies. Migration factors depend on both contextual or situation-specific factors as well as individual differences (Martin, 1993). Along with motives for migration, therefore, the current study also sought to examine the psychological processes involved with an individual's motivation or intentions to relocate and live in a new cultural environment.

Migration motivation and adjustment outcomes. Psychological research has examined the concept of motivation from various theoretical perspectives and across interrelated constructs (Winchie \& Carment, 1989). For example, motivation has been likened to and interchanged with variables such as personal agency, self-efficacy, selfregulation, and persistence (Gezentsvey \& Ward, 2008; Ryan \& Deci, 2000). What these 
overlapping constructs have in common is that each may be considered as an intention to act. "Motivation concerns energy, direction, persistence, and equifinality-all aspects of activation and intention" (Ryan \& Deci, 2001, p. 69). Migration motivation, then, can be viewed as an individual's intention to migrate to another culture or country. Based on evidence from the meta-analysis of the motivation-adjustment link as well as calls from researchers for more empirical investigation on the topic (Chirkov et al., 2007; Rumbaut, 1991; Schmitz, 2001), two main motivation constructs will be considered under the umbrella term of migration motivation: Autonomous regulation and Motivational CQ, a component of cultural intelligence.

Autonomous regulation. This perspective on motivation is derived from Deci and Ryan's Self-Determination Theory (Deci \& Ryan, 2000; Ryan \& Deci, 2001). The SelfDetermination Theory or SDT approach suggests that a person's innate psychological needs and growth tendencies form the base of their self-motivation (Ryan \& Deci, 2000). The theory postulates that behaviours derived from an individual's inherent values or interests (e.g., volitional or autonomously regulated behaviours) will result in better performance outcomes when compared to behaviours enacted as a result of external reasons (e.g., behaviours controlled or regulated by the values and interests of others).

Comparisons between people whose motivation is authentic (literally, selfauthored or endorsed) and those who are merely externally controlled for an action typically reveal that the former, relative to the latter, have more interest, excitement, and confidence, which in turn is manifest both as enhanced performance, persistence, ... and general well-being [Ryan, Deci, \& Grolnick, 1995]. This is so even when the people have the same level of perceived competence or self-efficacy for the activity (Ryan \& Deci, 2000, p. 69).

The SDT framework outlines four types of behavioural regulation (intrinsic, identified, introjected, and external regulation) that fall along a self-determination continuum that will be described in further detail as an autonomy index. Along this continuum, behaviours can range from those that fully embody self-determination (intrinsic regulation) to those that are entirely lacking in it (external regulation). Returning to migration factors as an example of the self-determination continuum, migrants who move to a new country based on pull motives (e.g., lifestyle factors) could be placed nearer the intrinsic or autonomouslyregulated behaviours side of the continuum, as they would have made the choice to relocate 
based upon their own interests and values. In contrast, individuals who relocate internationally due to push factors (e.g., preservation motives) would be seen to fall along the external regulation end of the continuum, as their move would be seen to be predicated upon the interests and values or others or external circumstances beyond their control.

The SDT paradigm has been applied across several disciplines including education, health care, and management/work settings to investigate how controlling versus autonomous regulation impacts on behavioural performance and competencies. For example, one SDT study primed participants' autonomous and controlled motivations prior to their engagement in a behavioural task they had never performed before. Participants who were primed for autonomous motivation learned the task more quickly and were more competent in the task than those participants primed for controlled motivation (Radel, Sarrazin, \& Pelletier, 2009). Additional studies utilising SDT have also evidenced the influence of autonomous regulation on behaviours such as work performance (Baard, Deci, \& Ryan, 2004) and academic competence (Houlfort et al., 2002; Vansteenkiste et al., 2010).

In addition to its impact on behavioural competencies, autonomous regulation has also been found to relate to psychological well-being. In a clinical setting of individuals experiencing depression, for example, participants who freely chose to receive treatment (intrinsic or autonomous regulation) reported better remission rates and lower post-treatment depression than those individuals who were forced or compelled to undergo treatment (e.g., controlled or extrinsic motivation; Zuroff et al., 2007). Within an academic domain, students' intrinsic self-regulation has been found to predict positive affect (Burton, Lydon, D'Alessandro, \& Koestner, 2006), enjoyment of elementary school and positive coping strategies (Ryan \& Connell, 1989), and positive mood (Reis et al., 2000). Numerous other studies have also evidenced a positive relationship between intrinsic or autonomous regulation and well-being outcomes such as happiness (e.g., Kasser \& Ryan, 1996; Sheldon \& Elliot, 1999; Koestner, Lekes, Powers, \& Chicoine, 2002).

The SDT framework has only recently been applied to the phenomena of migration motivation and cross-cultural adjustment. Research conducted by Chirkov and associates (e.g., Chirkov et al., 2004; Chirkov et al., 2007), for example, has focussed on how selfdetermined motivation differs in international students' intentions to study abroad. Studies within their research programme have demonstrated that self-determined migration motivation was predictive of various cultural adaptation outcomes such as greater subjective well-being, higher life satisfaction, and less depressive symptoms (Chirkov et al., 2007; Chirkov, Safdar, de Guzman, \& Playford, 2008). Associated research regarding Chinese 
sojourners in Canada has also indicated that well-being was positively predicted by these individuals' feelings of autonomy (Vansteenkiste et al., 2006). Using SDT, the present research builds upon the foundation begun by Chirkov and colleagues through continued examination of autonomous regulation and adjustment outcomes. Specifically, it is believed that a migrant's self-motivated interest in moving to New Zealand will be positively and significantly related to greater sociocultural competency, higher life satisfaction, and less depression.

Motivational CQ. As a component of cultural intelligence or CQ, Motivational CQ is another aspect of motivation that relates to an individual's motivational capacity to direct energy and awareness towards functioning in culturally diverse situations (Ang et al., 2007). Ang and colleagues formulated their conceptualisation of Motivational CQ based in part on Deci and Ryan's theory of intrinsic motivation (Deci \& Ryan, 1985) and Bandura's selfefficacy framework (2002). In particular, people with higher degrees of Motivational CQ are thought to experience greater cross-cultural effectiveness because they are more intrinsically motivated and confident in their abilities to operate within diverse cultural situations. As such, both confidence and intrinsic motivation are "intertwined because people choose to engage in activities when they feel efficacious" (Van Dyne et al., 2012). In turn, the CQ framework suggests that a person's motivation and ability to function in a new culture and the self-efficacy or confidence they have to do so creates greater incentives for success and enables perseverance through potential difficulties:

Since intercultural interactions can be stressful [Mendenhall and Oddou, 1985], motivational CQ... [has] special relevance to cultural adaptation. This is consistent with meta-analytic findings that self-efficacy and relationship skills predict expatriate adjustment [Bhaskar-Shrinivas et al., 2005]. Motivational CQ should positively relate to cultural adaptation because those with higher motivational CQ have intrinsic interest in other cultures and expect to be successful in culturally diverse situations. According to social cognitive theory [Bandura, 2002], they initiate effort, persist in their efforts and perform better (Ang et al., 2007, p. 342).

Motivational CQ has been linked to a variety of both behavioural competencies and well-being outcomes. For example, empirical work on the construct has found associations between Motivational CQ and effective management behaviours, increased leadership skills, 
expatriate job performance and longer overseas assignment stays, and other measures of organisational performance (Abdul Malek \& Budhwar, 2012; Earley, 2002; Matear, 2009; Vedadi, Kheiri, \& Abbasalizadeh, 2010). Further, in a sample of over 500 expatriates, Chen and colleagues (2010) reported that employees' Motivational CQ was significantly related to adjustment within their new international assignments, which in turn contributed to higher job performance. Templer, Tay, and Chandrasekar (2006) have also studied the influence of Motivational CQ on cross-cultural adaptation indicators such as work, general, and interaction adjustment in expatriates. They found that employees with greater degrees of interest in and motivation to experience new cultures and who were more confident in their abilities to be cross-culturally effective were better adjusted to social, life, and work demands while on their foreign assignments. Other researchers have suggested that out of all the CQ components, Motivation CQ is the best predictor of task performance (Chen, Liu, \& Portnoy, 2012) because it provides agentic control of both cognitive and behavioural processes that influence goal accomplishment.

With regard to psychological adjustment, Ang and associates' (2007) validation research found that Motivational CQ was associated with participants' satisfaction concerning their ability to concentrate, make decisions, and follow through with their responsibilities. In a sample of international students, this motivation component was also found to predict life satisfaction and sociocultural adaptation and, moreover, offered more explanatory power in the prediction of cross-cultural adaptation than other components of CQ (Ward, Fischer, Lam, \& Hall, 2008; Ward, Wilson, \& Fischer, 2011). Overall, literature regarding the effects of Motivational CQ on cross-cultural adjustment appears to suggest that those with higher degrees of confidence and intrinsic motivation to operate in diverse settings have a greater capacity to understand the cultural backgrounds of people in their host societies, are more able to establish interpersonal relationships with their cross-cultural counterparts, and are more competent in "real-world" problem solving (Van Dyne et al., 2012). It is therefore suggested that Motivational CQ will be positively related to both sociocultural and psychological adjustment, and associated with lower levels of perceived discrimination.

\section{Perceived Discrimination and Adjustment}

Perceived discrimination relates to an individual's subjective experience of various forms of unfair treatment due to status (e.g., socioeconomic, racial, gender). These experiences can include rejection of housing applications, the inability to obtain employment, 
and verbal or physical maltreatment (Kessler, Mickelson, \& Williams, 1999). A relatively large amount of literature has been published regarding the effects of perceived discrimination on psychological well-being. For example, a meta-analysis comprised of over 100 studies of ethnic or racial discrimination against Latino/as in the United States found that mental health indicators such as acculturative stress were most strongly correlated with discrimination (Lee \& Ahn, 2011). Another meta-analytic review on discrimination investigated its effect on physical health symptoms such as substance abuse, good health habits, and mental health outcomes including well-being and psychological stress (Pascoe \& Smart Richman, 2009). Not only did results of the meta-analysis provide an overview of the strong relationships between various forms of discrimination, mental well-being, and health outcomes, it also examined causal pathways among these variables. In particular, Pascoe and Smart Richman found that the association between perceived discrimination and well-being (both mental and physical) was mediated through behaviours such as exercise and diet. Other studies have found relationships between experiences of racial or ethnic discrimination and poor psychological well-being such as greater degrees of loneliness, anxiety, distress (Neto \& Barros, 2000; Revollo, Qureshi, Collazos, Valero, \& Casas, 2011), and depression (Ward, Berno, \& Main, 2002; Vedder, Sam, \& Liebkind, 2007).

Less empirical attention has been paid to the possible influence discrimination may exert on the behavioural competencies of migrants or ethnic minority populations. However, research focussing on adolescent minorities with regard to these variables has found positive correlations between discrimination and conduct problems (Oppedal, Røysamb, \& Heyerdahl, 2005), school tardiness and drug- and alcohol-related police involvement (Sabatier \& Berry, 2008), poor school adjustment (Liebkind, Jasinskaja-Lahti, \& Solheim, 2004; Vedder, Sam, \& Liebkind, 2007), and defensive self-presentation (Dion \& Earn, 1975). One study (Runions, Priest, \& Dandy, 2011) of Australian children from MiddleEastern and Asian backgrounds investigated their experiences of discrimination in relation to both psychological and social adjustment. Using self-report questionnaires from both the children and their parents, results of the study indicated that more than $85 \%$ of the children reported some form of discrimination against them, and that these experiences of discrimination were predictive of impaired social functioning in terms of withdrawn social behaviour and aggression. Last, as was previously described, meta-analytic results from Chapter 2 found 6 studies reported strong associations between higher levels of discrimination and sociocultural adaptation difficulties as measured specifically by the SCAS (e.g., Gungor \& Bornstein, 2009; Zlobina, Basabe, Paez, \& Furnham, 2006). When 
considered together, these studies suggest that a positive relationship between perceived discrimination and depression, and negative associations between discrimination, life satisfaction, and behavioural competency will be found in the hypothesised path models.

\section{Motivation and Perceived Discrimination}

The previous sections introduced research involving the migration motivationadjustment and discrimination-adjustment relationships. As no empirical research was discovered that has specifically examined the linkages between reasons for migration and migration motivation (as measured by Motivational CQ and autonomous regulation) and perceived discrimination, theoretical rationale for investigation of the potentially causal pathways between these variables was derived from the CQ and SDT frameworks as well as Lazarus and Folkman's cognitive appraisal model (1984).

First, the CQ and SDT paradigms posit that motivation is related to an individual's values, beliefs in their competence, ability to cope with life demands, and determination in goal attainment. For example, a host of studies examining autonomous regulation have found it was related to greater perseverance (Pelletier, Fortier, Vallerand, \& Brière, 2001; Vallerand \& Bissonnette, 1992; Vallerand, Fortier, \& Guay, 1997), greater intention to persist (Hardre \& Reeve, 2003; Noels, Pelletier, Clément, \& Vallerand, 2000), and more determination and will as measured by greater effort-expenditure (Ryan \& Connell, 1989). In addition, individuals with higher levels of Motivational CQ are thought to be confident in social interactions and interpersonally adaptive within new cultural environments, may seek to develop relationships with culturally-different individuals (Earley \& Ang, 2003), and have been shown to exhibit greater interaction adjustment (Harrison et al., 1996; Hechanova et al., 2003). Hence, theoretical and empirical literature supports the view that a person who values diversity and is self-motivated to experience different cultures may have more confidence or higher self-efficacy in their ability to persevere through difficulties, stressors, or challenges that arise within a host society.

The second aspect of the theoretical argument posed regarding the motivationdiscrimination link is based on Lazarus and Folkman's cognitive appraisal model (1984), which suggests that perceived discrimination may be considered as a type of environmental stressor. The cognitive appraisal model proposes that individual psychological factors influence how a person perceives, evaluates or appraises, and copes with environmental stressors or threats. Theorists (e.g., Feldman Barrett \& Swim, 1998; Pascoe \& Smart Richman, 2009) have proposed that an individual's perception of prejudice or discrimination 
is a type of stressor or threat appraisal, and several empirical studies have applied this view of racism, prejudice, or discrimination to a psychological stress and coping framework in the examination of its influence on various health and well-being outcomes (see Allison, 1998; Clark, Anderson, Clark, \& Williams, 1999; Contrada et al., 2000; Major, Quinton, \& McCoy, 2002; Williams et al., 1999). Furthermore, other studies have found relationships between autonomous motivation and efficacious beliefs and lowered threat response (Hodgins et al., 2010), as well as appraisals of discrimination (Cassidy \& O'Connor, 2005; Hassel \& Perrewé, 1993; Phinney, Madden, \& Santos, 1998). With these two frameworks and empirical evidence in mind, it is suggested that an individual's self-motivated interest, and confidence in his or her ability to function effectively in a new culture may lower their perceptions of discrimination. Specifically, the underlying mechanism of this hypothesised link may be that confident and determined individuals may appraise potential discriminatory actions against them as a stressor or difficulty that can be overcome.

\section{Behavioural Competency and Psychological Adjustment}

A variety of multi-disciplinary research has investigated the influence and mediating effects of various aspects of behavioural competency on psychological well-being. In the medical field, some studies such as one conducted by Smith and colleagues (Smith, Dobbins, \& Wallston, 1991) have found evidence that perceived competence acted as a mediator between individuals' experience of rheumatoid arthritis and psychological adjustment as measured by depressive symptoms and life satisfaction. Specifically, these researchers argue that development of interventions aimed at increasing an individual's generalised sense of competency may facilitate both their psychological and functional well-being. In the developmental literature, research has uncovered mediational effects of competence on the relationship between perceptions of threat and depression in African American youth (Prelow, Weaver, \& Swenson, 2006), and children exposed to domestic violence and their psychological adjustment (Katz, Hessler, \& Annest, 2007). Further, a longitudinal study of shy-anxious Spanish-speaking preschoolers in the United States found that communication competence-based on teacher-report items of procedural and pragmatic aspects of communicating with others-impacted the relationship between children's ability to express themselves and their levels of anxiety (Strand, Pula, Parks, \& Cerna, 2011).

Research within the culture contact literature has also focussed on the potential role behavioural competence plays in the psychological well-being of various migrant groups. One study involving South Asian international students at an American university found that 
more instances of self-reported intercultural behaviours predicted lower levels of depression (Rahman \& Rollock, 2004). Another longitudinal study of Russian and Estonian immigrants in Finland found that sociocultural adaptation — as measured by language competency—was the most significant predictor of better psychological well-being (measured by less stress, anxiety, and depression) after 8 years of residence (Jasinskaja-Lahti, 2008). Further, Dalhaug and colleagues (Dalhaug, Oppedal, \& Røysamb, 2011) investigated host culture competence and depression in two multicultural schools. They postulated that acquisition of "host cultural competence is a product of interpersonal interaction and is important to adaptation and well-being” (p. 283), and in support of their hypotheses a negative association was found between cultural competence and depression. Last, the International Comparative Study of Ethno-cultural Youth or ICSEY project (Berry, Phinney, Sam, \& Vedder, 2006) also examined various intercultural variables such as language competency and social contact with both ethnic peers and members of other ethnic groups as predictors of psychological adjustment. These findings form the foundation of the present study's hypotheses that greater sociocultural competency reported by migrants resident in New Zealand will be predictive of higher levels of life satisfaction and fewer reports of depression.

\section{The Potential Mediating Effects of Perceived Discrimination}

A novel approach in the acculturation literature is now explored where perceived discrimination is suggested to mediate the relationship between behavioural competency (as measured by the SCAS-R) and psychological adjustment. It is proposed that an individual's behavioural competence is an important determinant of the degree of discrimination he or she perceives and experiences, and that in turn the amount of discrimination a person perceives influences his or her psychological adjustment to a new culture. The following section will review empirical evidence that has attempted to elucidate the various direct and indirect relationships between these constructs of behavioural competency, discrimination, and psychological well-being.

Some acculturation research has examined how behavioural competencies influence an individual's experience of discrimination. For example, Neto (2006) conducted a study with various immigrant groups in Portugal and found that behavioural problems and stressful adaptation experiences as measured by the SCAS predicted immigrants' experiences of discrimination. Further, a path-analytic approach taken by Phinney and colleagues (1998) found that adolescent migrants to the United States who reported greater feelings of competence socialising with people in intergroup situations also experienced significantly 
less perceived discrimination. Another study on cultural competence conducted by Oppedal, Røysamb, and Sam (2004) posited that an increase in migrant students' behavioural competence within their host society would be associated with a decrease in perceived discrimination. Indeed, the path analysis found that students' host culture competence was negatively associated with the discrimination they reported experiencing in class. Oppedal and colleagues concluded their study by commenting that the "notion of culture competencies may be as important to understanding the health issues of diverse ethnic groups as the demographic information that is typically collected as indicators of acculturation", such as ethnicity and host country residency (p. 492).

Some studies of acculturation have also concentrated on the indirect effects of behavioural competence on the relationship between discrimination and psychological wellbeing. For example, Wei and colleagues (2010) examined stress that Asian international students studying in the United States experienced due to discrimination, and reported that non-reactive coping behaviours in the students' new cultural environment reduced the strength of the relationship between racial discrimination stress and depressive symptoms they reported. Additionally, in support of the present study's supposition that greater behavioural cultural competency will predict less discrimination and in turn better psychological adjustment, Torres (2007) studied the contributions of acculturation, ethnic identity, coping, and intercultural competence in predicting depression among Hispanic adults. He found that intercultural competency difficulties in developing effective personenvironment relationships affected the relationship between acculturation and depression. Torres further suggests that competence- and ability-based variables should be integrated into psychological conceptualisations of cultural adaptation. In consideration of these findings, greater cross-cultural behavioural competency as measured by the SCAS-R is therefore expected to predict less perceived discrimination and in turn more positive psychological adjustment (e.g., higher levels of life satisfaction and lower levels of depression).

\section{Study Overview and Hypotheses}

The present study continued investigation of the psychometric and conceptual underpinnings of the SCAS-R and examined the relationships between migration factors, migration motivation, cultural competency, perceived discrimination, and psychological adjustment. The first objective of the study involved confirmation of the SCAS-R factor structure and further corroboration of the instrument's internal consistency. As such, it was expected that: 
1. A CFA will confirm the existing five-factor structure of the SCAS-R.

2. The SCAS-R and its five subscales will demonstrate acceptable reliability.

The second aim of the current study was to examine the potentially causal relationships between motivation factors, migration motivation, sociocultural adaptation competency, discrimination, and psychological adjustment. As was previously outlined, a number of studies have considered the linkages between these variables. Migration factors, autonomous regulation, and Motivational CQ have been linked to positive behavioural and psychological outcomes (e.g., Chirkov, Safdar, de Guzman, \& Playford, 2008; Funham \& Bochner, 1986; Ward, Wilson, \& Fischer, 2011). Behavioural competencies have been associated with less perceived discrimination (Oppedal, Røysamb, \& Sam, 2004) as well as greater psychological adjustment (Berry, Phinney, Sam, \& Vedder, 2006). Furthermore, some work has found that perceived discrimination mediated the effects of competency on adjustment (Wei, Heppner, Ku, \& Liao, 2010). Despite this extant research on motivation, behavioural competency, discrimination, and psychological well-being, no literature was discovered investigating the simultaneous associations among these concepts. Based on the aforementioned research, the following assumptions about the direct and indirect pathways between these four constructs have been illustrated in the hypothetical path models presented at the beginning of the chapter (Figures 4 and 5).

\section{Method}

\section{Procedure}

A survey was developed for the study and its electronic version made available online using a data collection website. E-mails were sent to two government organisations engaged with migrant communities asking key contact persons (e.g., settlement support coordinators, community liaisons) to provide their clientele with information about the research via e-mail, listserv, newsletter, or mailing list. Further, the e-mail listed the following inclusion criteria: Eligible participants were to be expatriates or migrants aged 16 years and older, born outside of New Zealand, and living in the country for five or less years ${ }^{2}$. A link to the survey was also included in the e-mail.

2 As mentioned in the previous chapter, this time period was selected due to literature suggesting that behavioural adaptation occurs during initial entry into a host society and 
Approximately two months later, follow-up e-mails were sent to the government contacts as a reminder of the survey closing date. Throughout the research, participants were never directly contacted by the researcher. Data collection was open from 29 November 2010 to 15 May 2011.

\section{Participants}

An initial total of 202 individuals completed the survey. Of these, participants were removed from the analysis due to living outside of New Zealand $(N=5)$, living in New Zealand for over five years $(N=4)$, and failing to complete over $80 \%$ of the survey $(N=10)$, bringing the final total of participants to 185 .

From the 185 respondents, 133 were women and 49 were men (missing $=3$ ); $\mathbf{M}_{\text {age }}=$ $39.20, \mathrm{SD}=9.04$, age range $=17-67$ years. The majority of participants described their ethnic identities according to the Statistics New Zealand ethnicity classification system (Review of the Measurement of Ethnicity, 2004) as European (62.7\%), Asian (24.9\%), Middle Eastern/Latin American/African (8.1\%), or Pacific (1.6\%). A full list of participant demographics including nationalities and ethnicities is detailed in Appendix E.

A large portion of migrants were highly educated: 76 or $42 \%$ listed having received a postgraduate degree (e.g., Master's or $\mathrm{PhD}$ ), and 64 (35.4\%) reported holding a tertiary degree. Post-secondary certificates or diplomas were held by $9.4 \%$, followed by vocational qualifications or trade certificates (7.2\%). A smaller percentage of participants reported their highest qualification as secondary school $(6.1 \%)$.

Regarding language fluency, $28.2 \%$ of participants described their current overall English language proficiency (reading, writing, understanding, and speaking) as excellent ( $N$ $=51)$; above average $(N=30)$; average $(N=4)$; and below average $(N=2)$. Over half of the participants were native English speakers $(50.8 \%$ or $N=94)$.

Participants reported having lived in New Zealand for an average of just over two years $(M=26.38$ months, $\mathrm{SD}=18.71)$, and most expected to remain in the country permanently $(53 \%)$, followed by five years or more $(22.1 \%)$, between three and five years (11.6\%), and between one and three years $(9.9 \%)$. Just over three percent of participants intended to leave within the year. From those participants who intended to leave New Zealand eventually, $31.3 \%(N=36)$ planned to return to their home country. Of those who indicated that they would migrate to a country other than their country of origin $(N=79)$, the

levels off gradually as new cultural skills are acquired (Furnham \& Bochner, 1986; Ward \& Kennedy, 1999; Ward et al., 1998; Ward \& Rana-Deuba, 1999). 
majority listed countries such as Australia $(N=9)$ and England $(N=4)$, or were undecided between multiple countries.

Last, of the 177 individuals who responded to an item concerning work status, 89 were full-time employees, followed by full-time international students $(N=35)$, part-time employees $(N=33)$, part-time international students $(N=11)$, unemployed students $(N=7)$, and students who also worked full-time $(N=2)$.

\section{Materials}

The 30-minute survey included demographic items (e.g., age, gender, nationality), motivation (migration factors and migration motivation), perceived discrimination, and crosscultural adjustment measures (see Appendix F for survey). All measures except for the revised SCAS and demographics were previously published. The means, standard deviations, and intercorrelation matrix of the demographic variables and included measures are presented in Table 8 .

Migration factors and migration motivation. Three measures regarding migrants' motivations for moving to New Zealand were included in the survey. The first, measuring migration factors, was a revised 15-item Department of Labour questionnaire from their Settlement Report (IMSED, 2008) which listed specific reasons that may have motivated participants' moves to New Zealand. These reasons included "Marry or live with a spouse or partner", "Employment opportunities", and "Safety from crime". Participants were asked to rate the extent to which each of the items applied to them personally $(1=$ Not at all; $5=$ Very mисh). The scale was factor analysed, and the factors were discovered to relate to migration factors of preservation or MFP (6 items; $\alpha=.81$ ), lifestyle or MFL (three items; $\alpha=.83$ ), and familial or MFF (three items; $\alpha=.60$; average inter-item correlation $r=.34$ ).

Cultural Intelligence Scale Motivational CQ. Developed by Ang and colleagues (2007), the motivational component of the Cultural Intelligence Scale or CQM examines an individual's internal motivation, interest, and confidence in functioning effectively within different cultural settings on a 5-point agree-disagree scale where higher scores reflect a higher degree of motivation. The internal consistency of the CQM and the four-component structure of the overall CQ measure has been demonstrated in a number of studies (Ang et al., 2004; Van Dyne, Ang, \& Koh, 2008; Templer, Tay, \& Chandrasekar, 2006; \& Ward, Fischer, Lam, \& Hall, 2009). Cronbach's alpha for the CQM in this study was .91.

Self-Regulation Questionnaire for Study Abroad. A second migration motivation measure was adapted from Chirkov and colleagues' Self Regulation Questionnaire for Study 
Abroad or SRQ-SA (2007). The SRQ-SA items distinguish between four types of motivation regulation: Intrinsic, introjected, identified, and external regulation. Intrinsic motivation reflects a migrant's belief that their move to New Zealand was motivated by interest, excitement, or was a challenging opportunity (e.g., "I moved to New Zealand because I thought it would be an exciting thing to do."). A migrant's personal commitment to having moved to New Zealand typifies identified motivation (e.g., "I moved to New Zealand because it was of great personal value to me."). The third motivation type, introjected motivation, refers to the internal pressures migrants place upon themselves to meet the expectations of others, such as approval-seeking and guilt avoidance (e.g., "I moved to New Zealand because I would be criticized if I did not."). External regulation is related to the external pressures migrants may experience, such as life circumstances or spousal expectations (e.g., "I moved to New Zealand because others [spouse, family, friends] were pushing me to do this."). A total of 20 items representing these four motivation typologies were rated on a Likert scale from 1 (= Not at all) to 5 (=Very much).

The four sub-scales of the SRQ-SA were ordered using a simplex correlation pattern to calculate the Relative Autonomy Index or AUTO (see Chirkov et al., 2007). The Autonomy Index is a bi-directional scale of autonomous regulation, a combination of the intrinsic motivation and identified regulation sub-scales, and controlled regulation (external and introjected motivation). Positive scores on the AUTO reflect a higher occurrence of autonomous regulation, and negative scores reflect controlled regulation. In this study, the AUTO mean score was $6.58(\mathrm{SD}=3.68) ; \min =-9.77$ and $\max =12$. 
Table 8

Means, Standard Deviations, and Zero-Order Correlations Among Variables $(N=146)$

\begin{tabular}{|c|c|c|c|c|c|c|c|c|c|c|c|c|c|c|c|}
\hline Variable & $M$ & $S D$ & 1 & 2 & 3 & 4 & 5 & 6 & 7 & 8 & 9 & 10 & 11 & 12 & 13 \\
\hline $\begin{array}{l}\text { 1. SCAS-R } \\
(.88)\end{array}$ & 3.97 & .51 & --- & -.08 & .06 & $.19 *$ & $.35 * *$ & $.30 * *$ & $-.19 *$ & $.40 * *$ & $-.48 * *$ & .03 & $.35 * *$ & -.04 & .10 \\
\hline $\begin{array}{r}\text { 2. MFF } \\
(.60)\end{array}$ & 1.93 & 1.11 & & --- & -.13 & -.10 & -.03 & $-.31 * *$ & -.02 & -.11 & .12 & -.09 & -.09 & $-.18 *$ & $-.18 *$ \\
\hline $\begin{array}{r}\text { 3. MFP } \\
(.81)\end{array}$ & 2.89 & 1.08 & & & --- & $.44 * *$ & -.10 & .05 & -.04 & .08 & -.07 & .06 & $-.19 *$ & $.28 * *$ & .09 \\
\hline $\begin{array}{l}\text { 4. MFL } \\
(.83)\end{array}$ & 3.75 & 1.03 & & & & --- & -.09 & $.44 * *$ & $-.22 * *$ & $.28 * *$ & $-.28 * *$ & -.06 & .04 & .13 & .03 \\
\hline $\begin{array}{l}\text { 5. CQM } \\
(.91)\end{array}$ & 5.52 & 1.21 & & & & & --- & $.19^{*}$ & -.12 & $.27 * *$ & $-.28 * *$ & -.04 & -.04 & -.04 & .01 \\
\hline $\begin{array}{l}\text { 6. AUTO } \\
\text { N/A }\end{array}$ & 6.44 & 3.68 & & & & & & --- & $-.19 *$ & $.44 * *$ & $-.40 * *$ & -.13 & $.18^{*}$ & .04 & .12 \\
\hline $\begin{array}{l}\text { 7. PD } \\
(.91)\end{array}$ & 1.51 & .60 & & & & & & & --- & $-.41 * *$ & $.44 * *$ & $.39 * *$ & -.11 & .03 & -.01 \\
\hline $\begin{array}{l}\text { 8. SWLS } \\
(.87)\end{array}$ & 3.62 & .86 & & & & & & & & --- & $-.62 * *$ & -.06 & .15 & -.03 & -.01 \\
\hline $\begin{array}{c}\text { 9. ZSDS } \\
(.85)\end{array}$ & 1.76 & .47 & & & & & & & & & --- & $.27 * *$ & $-.20 *$ & -.08 & -.05 \\
\hline $\begin{array}{l}\text { 10. LOR } \\
\text { N/A }\end{array}$ & 26.22 & 18.71 & & & & & & & & & & --- & .10 & -.05 & .04 \\
\hline $\begin{array}{l}\text { 11. ELP } \\
\text { N/A }\end{array}$ & 5.25 & .90 & & & & & & & & & & & --- & -.06 & .12 \\
\hline $\begin{array}{l}\text { 12. Gender }(1= \\
\text { Female })\end{array}$ & --- & --- & & & & & & & & & & & & --- & .01 \\
\hline 13. Age & 38.50 & 1.16 & & & & & & & & & & & & & --- \\
\hline
\end{tabular}

Note. Cronbach's alphas are displayed in parentheses where applicable. Correlations between SCAS-R and other scales are in boldface. SCAS-R = Revised Sociocultural Adaptation Scale (21-item post CFA scale); MFF = Familial Migration Factors; MFP = Preservation Migration Factors; MFL = Lifestyle Migration Factors; CQM = Motivational CQ; AUTO = Relative Autonomy Index; PD = Perceived Discrimination; SWLS = Satisfaction with Life Scale; ZSDS = Zung Self-Rating Depression Scale; LOR = Length of Residence in New Zealand; ELP = English Language Proficiency.

$* p<.05 . * * p<.01$. 
Perceived discrimination. The present study utilised a modified version of Williams and colleagues' (1999) measure of perceived discrimination or PD $(\alpha=.88)$, which focusses on individuals' routine or day-to-day experiences of unjust treatment rather than the race or ethnicity of the respondent. Participants answered items such as how frequently they "have experienced less respect than others", they "were threatened or harassed", or "received poorer service than others in restaurants and stores" on a 4-point scale $(1=$ Never; $4=$ Often). The internal consistency of the measure in the present study was .91.

Cross-cultural adjustment. The following instruments were used as measures of sociocultural adaptation and psychological adjustment.

Sociocultural adaptation. The Revised Sociocultural Adaptation Scale (SCAS-R) requires participants to rate their level of sociocultural competence with a variety of behaviours such as "Maintaining my hobbies and interests", "Building and maintaining relationships", "Changing my behaviour to suit social norms, rules, attitudes, beliefs, and customs", and "Working effectively with other students/work colleagues". Higher scores (1= Not at all competent; 5 = Extremely competent) were indicative of greater sociocultural adaptation competency. Study 2 results from this research programme indicated a Cronbach's alpha of .92, and the scale's internal consistency in the present study remained acceptable ( $\alpha$ $=.88)$.

Psychological adjustment. Psychological adjustment was assessed using both negative and positive indicators; the Zung Self-Rating Depression Scale or ZSDS (Zung et al., 1965) and the Satisfaction with Life Scale or SWLS (Diener et al., 1985). The 19-item version of the ZSDS examines physiological, affective, and psychological components of depression. Individuals are asked to indicate on a 5-point scale how frequently (endpoints $1=$ A little of the time, $5=$ Most of the time) they experience various depression components, where higher scores represent greater depression. The ZSDS has been used extensively in cross-cultural studies (Zung, 1972; Zung et al., 1969,) and has proven a reliable instrument with high internal consistency in Ward and colleagues' research with multinational samples of sojourners (Ward \& Kennedy, 1993; Ward, Leong, \& Low, 2004). In this study, Cronbach's alpha was .85. The SWLS consists of five items including "I am satisfied with my life" that measure life satisfaction on a Likert scale of $1=$ Strongly disagree to $5=$ Strongly agree . This SWLS version has been utilised in 13 countries across 25 ethnic groups and has shown good validity and reliability (Berry, Phinney, Sam, \& Vedder, 2006). The internal consistency in this study was $\alpha=.87$. 


\section{Results}

There were two main purposes of the present study. One aim of the analyses was to confirm the factor structure of the revised SCAS developed in Study 2 utilising confirmatory factor analysis (CFA) and Cronbach's alpha to demonstrate continued evidence of its internal consistency. A second objective was to test two integrated models of motivation and psychological well-being. Specifically, potentially causal pathways between these variables including the double-mediation effects of sociocultural competency and perceived discrimination were examined utilising correlation and path analyses. The following section details these approaches and findings.

\section{Confirmatory Factor Analysis}

Confirmatory factor analysis has been used extensively in the psychology field for instrument validation (e.g., Byrne, 1989). To this end, the data were subjected to a CFA using the AMOS 18 program (Arbuckle, 2009) to test the proposed five-factor structure of the SCAS-R across an independent sample and for re-specification of its structure (Costello \& Osborne, 2005).

Based on results of the EFA from Study 2, the hypothesised CFA model (Figure 6) made the following a priori assumptions: (1) participant responses to the SCAS-R would be explained by five factors (Interpersonal Communication or IC; Academic/Work Performance or AWP; Personal Interests and Community Involvement or PICI; Ecological Adaptation or EA; and Language Proficiency or LP); (2) each item would have a non-zero loading on the factor it was designed to measure and zero loadings on all other factors; (3) the five factors would be inter-correlated; and (4) the error/uniqueness terms associated with the item measurements would be uncorrelated.

A missing value analysis was run on the data set, which found less than $5 \%$ of the sample to be missing completely at random or MCAR, $\chi^{2}=131.84[111], p=.09$. Therefore, regression imputation was utilised to impute any missing data. The hypothesised five-factor model was then tested with maximum likelihood estimation, as the sample and measure met normality assumptions, by loading each of the 21 scale items onto five latent factors. 


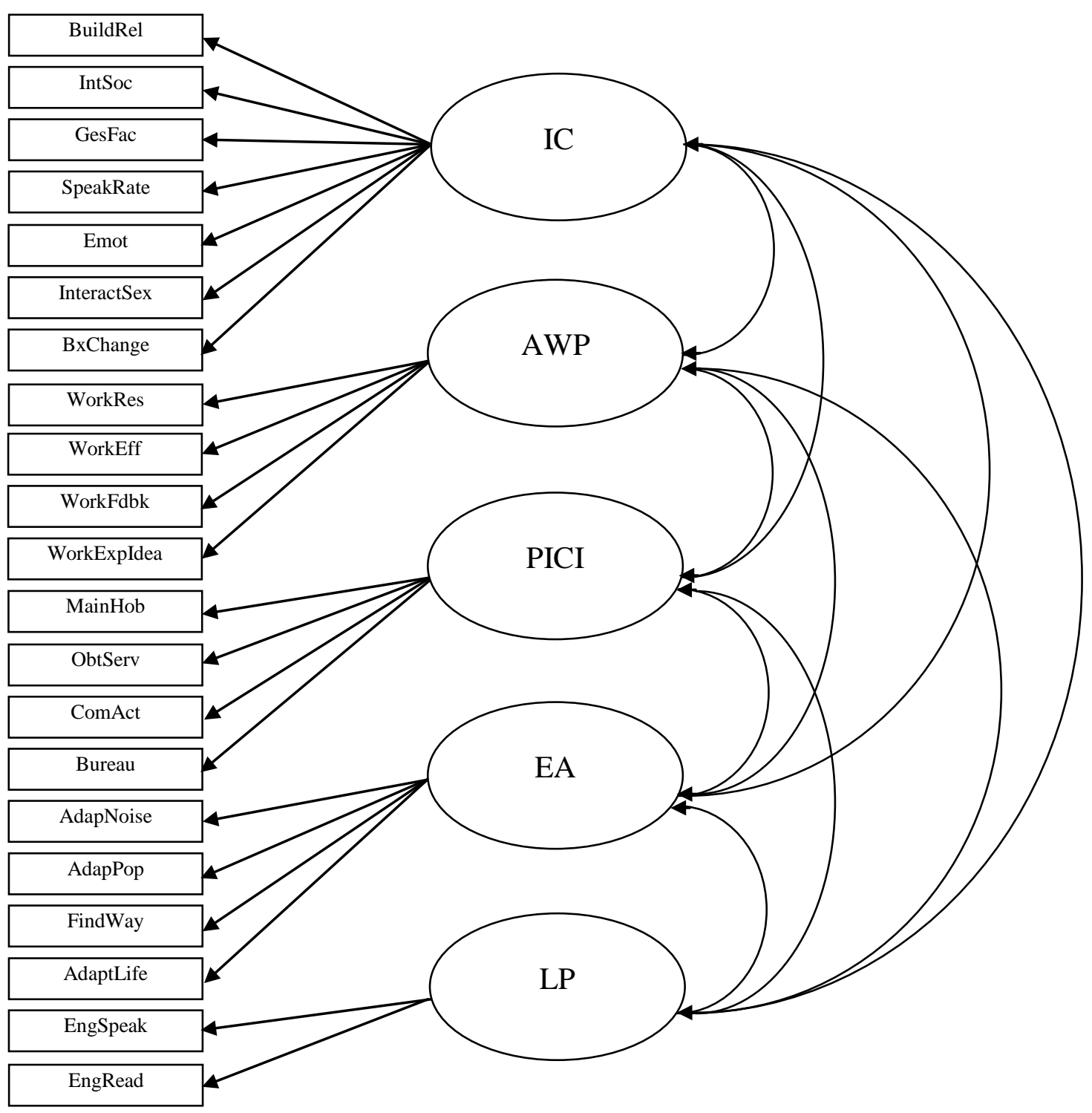

Figure 6. Hypothesised first-order CFA model of the SCAS-R.

Note. $\mathrm{IC}=$ Interpersonal Communication; AWP $=$ Academic/Work Performance; PICI $=$ Personal Interests/Community Involvement; EA = Ecological Adaptation; LP = Language Proficiency.

To ascertain the extent to which the initial model adequately represented the covariance matrix of the data, several goodness-of-fit indices were used, such as the chisquare statistic or $\chi^{2}$, the Comparative Fit Index (CFI), the Root Mean Square Error of Approximation (RMSEA), and the Standardised Root Mean Square Residual (SRMR). According to recommended cut-off values (Brown, 2006; Hu \& Bentler, 1999; Kline, 2010) the model fell below acceptable levels and therefore failed to meet the study's first hypothesis; $\chi^{2}(179)=508.20, p<.001, \mathrm{CFI}=.82, \mathrm{RMSEA}=.10$ with $\mathrm{CI}_{90}(.09, .11)$, and 
SRMR $=.07$. As these statistics were indicative of poor model fit, a specification search was required to identify a new model more representative of the data.

A re-specified model was created utilising both statistical and conceptual rationale. First, modification indices and standardised residuals were examined to determine significant discrepancies in the covariance between variables. Several scale items were found to have large correlated errors; therefore, four scale items correlating too highly with one another were removed: "Accurately interpreting and responding to other people's emotions", "Interacting at social events", "Adapting to the population density", and "Dealing with the bureaucracy".

Further, according to Brown (2006), covariance between the IC and AWP domains was quite high $(>.80)$. Conceptually, this finding may be attributed to the fact that the IC and AWP factors from the Study 2 EFA reflected behaviours in specific situational environments (e.g., interpersonal interaction behaviours and those behaviours within a work or academic setting). Although this conceptual configuration created sufficient model fit in the previous study's EFA, the large amount of factor covariance in the current study between the IC and AWP domains indicated incompatibility with the hypothesised SCAS-R fivefactor framework. Specifically, the high IC-AWP covariance suggested that a clear distinction does not exist between communication behaviours that occur within as opposed to across the proposed IC and AWP contexts. In other words, communication processes between individuals occur both inside and outside of a work/academic environment.

This theoretical rationale was used in order to justify re-specifications to the IC and AWP factors (Jackson, Gillaspy, \& Purc-Stephenson, 2009). The decision was made to move two items ("Gaining feedback from other work colleagues to help improve my performance" and "Expressing my ideas to other work colleagues in a culturally appropriate manner") from the AWP factor to the IC factor as these items related to communication behaviours. The revised IC factor was renamed "Communication", and the remaining two items in the AWP factor ("Managing work responsibilities" and "Working effectively with others") were deleted as they were not clearly related to communication processes.

Upon further examination of scale items remaining in the Communication factor, it was determined that several related more to maintaining, building, and/or being involved in relationships than communication processes. Consequently, a new factor titled "Involvement" was created, and the items "Building and maintaining relationships", "Interacting at social events", "Maintaining hobbies and interests", and "Participating in community activities" were shifted from the Communication factor to this new domain. The 
other two SCAS-R factors, Ecological Adaptation and Language Proficiency, were maintained in the re-specified model.

Deletion of the aforementioned items and creation of the Communication and Involvement factors greatly improved model fit in the post-hoc model (Figure 7) to acceptable standards, $\left(\Delta \chi_{(81)}^{2}=341.90, p<.001\right)$ RMSEA $=.06$ with $\mathrm{CI}_{90}(.05, .08), \mathrm{CFI}=$ 95. The final SCAS-R model was comprised of four factors or subscales: Communication (7 items); Involvement (four items); Ecological Adaptation (three items); and Language Proficiency (two items). All factor loadings and parameter estimates are detailed in Table 9. The study's second hypothesis concerning internal reliability was partially confirmed: The overall SCAS-R was found to have acceptable reliability as was previously reported $(\alpha=$ $.83)$, as were the refitted factors of Communication $(\alpha=.84)$, Involvement $(\alpha=.80)$, and Language Proficiency $(\alpha=.93)$. However, Cronbach's alpha of .60 for the Ecological Adaptation factor fell below the acceptable cut-off point of .70 suggested by Nunnally (1978). A table (Table 10) has been provided detailing these reliabilities as well as correlations between the SCAS-R subscales and the other study variables.

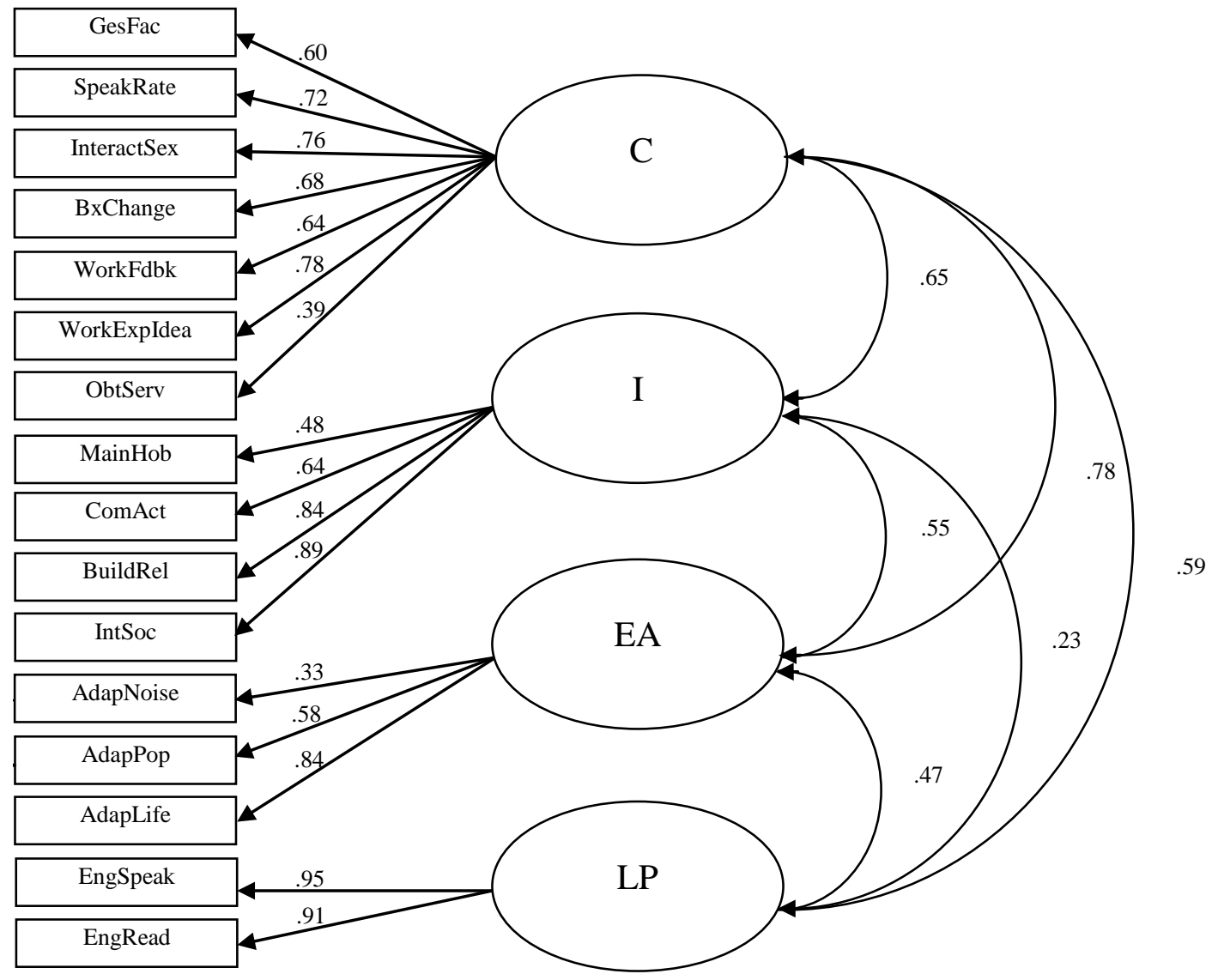

Figure 7. Re-specified first-order CFA model of the SCAS-R.

Note. Each observed variable has an associated error term not pictured here. $\mathrm{C}=$ Communication; I = Involvement; EA = Ecological Adaptation; LP = Language Proficiency. 


\section{Correlation Matrices}

Zero-order correlations (previously displayed in Table 8) among demographic, antecedent, mediator, and outcome variables were examined as a preliminary step in corroborating the relationships outlined in the hypothesised path models. In partial support of the first hypothesised path model (e.g., migrants' motives for moving abroad and psychological well-being), lifestyle factors were found to be significantly correlated with sociocultural adaptation $(r=.19)$, life satisfaction $(r=.28)$, and depression $(r=-.28)$. These results suggest that direct effects between lifestyle factors, behavioural competency, and psychological adjustment may exist. However, no significant correlations were discovered among preservation factors, sociocultural adaptation, or psychological well-being, providing evidence that this migration factor would perhaps not make a significant contribution to the hypothesised path model $^{3}$.

Correlations between migration motivation, sociocultural adaptation competency, discrimination, and psychological adjustment were also reviewed for evidence of potential significant pathways in the hypothesised migration motivation-psychological adjustment path model. Expectations regarding possible associations between autonomous regulation and Motivational CQ and the aforementioned variables were supported. Specifically, autonomous regulation was found to correlate significantly with the SCAS-R $(r=.30)$, PD ( $r$ $=-.19)$, SWLS $(r=.44)$, and ZSDS $(r=-.40)$. Furthermore, significant associations were discovered between CQM and the SCAS-R at $r=.35$ and psychological adjustment (SWLS $r$ $=.27$; ZSDS $r=-.28$ ). Suppositions regarding possible linkages between discrimination, behavioural competency, and psychological adjustment were also confirmed through negative correlations between the SCAS-R and SWLS ( $r$ s = -.19 and -.41, respectively), and a positive relationship with depression $(r=.44)$. A significant relationship was not found between CQM and PD, however, a finding that was in opposition to the hypothesised pathways.

3 Interestingly, no significant correlations were discovered between familial migration factors, sociocultural competency, discrimination, and psychological well-being. Although no hypotheses were formed for this particular migration factor, significant zero-order correlations between these variables would have supported inclusion of familial factors into the path model for exploratory, post-hoc analyses. 
Table 9

Confirmatory Factor Analysis of the SCAS-R and Factor Loadings of Items

\begin{tabular}{lcccccccc}
\hline Model & $\chi^{2}$ & $d f$ & $p<$ & $R M S E A$ & SRMR & $C F I$ & $\Delta \chi^{2}$ & $p$ \\
\hline 1. Five Factor & 508.20 & 179 & .0001 & .10 & .07 & .82 & \\
2. Four Factor & 166.30 & 98 & .0001 & .06 & .06 & .95 & $341.90>.001$ \\
\hline Items & & & & & Factor Loadings \\
\hline
\end{tabular}

Communication

6. Accurately interpreting and responding to other people's gestures and facial expressions.

8. Obtaining community services I require.

11. Varying the rate of my speaking in a culturally appropriate manner.

12. Gaining feedback from other work colleagues to help improve my performance.

16. Interacting with members of the opposite sex. $\quad .76$

17. Expressing my ideas to other work colleagues in a culturally appropriate manner.

21. Changing my behaviour to suit social norms, rules, attitudes, beliefs, and customs. .68

Б Involvement

1. Building and maintaining relationships.

3. Interacting at social events. $\quad .89$

4. Maintaining my hobbies and interests.

14. Attending or participating in community activities.

Ecological Adaptation

5. Adapting to the noise level in my neighbourhood.

9. Adapting to the population density.

19. Adapting to the pace of life. $\quad .84$

Language Proficiency

10. Understanding and speaking English. $\quad .95$

20. Reading and writing English. $\quad .91$ 
Table 10

Means, Standard Deviations, and Zero-Order Correlations Among SCAS-R Subscales and Study Variables $(N=146)$

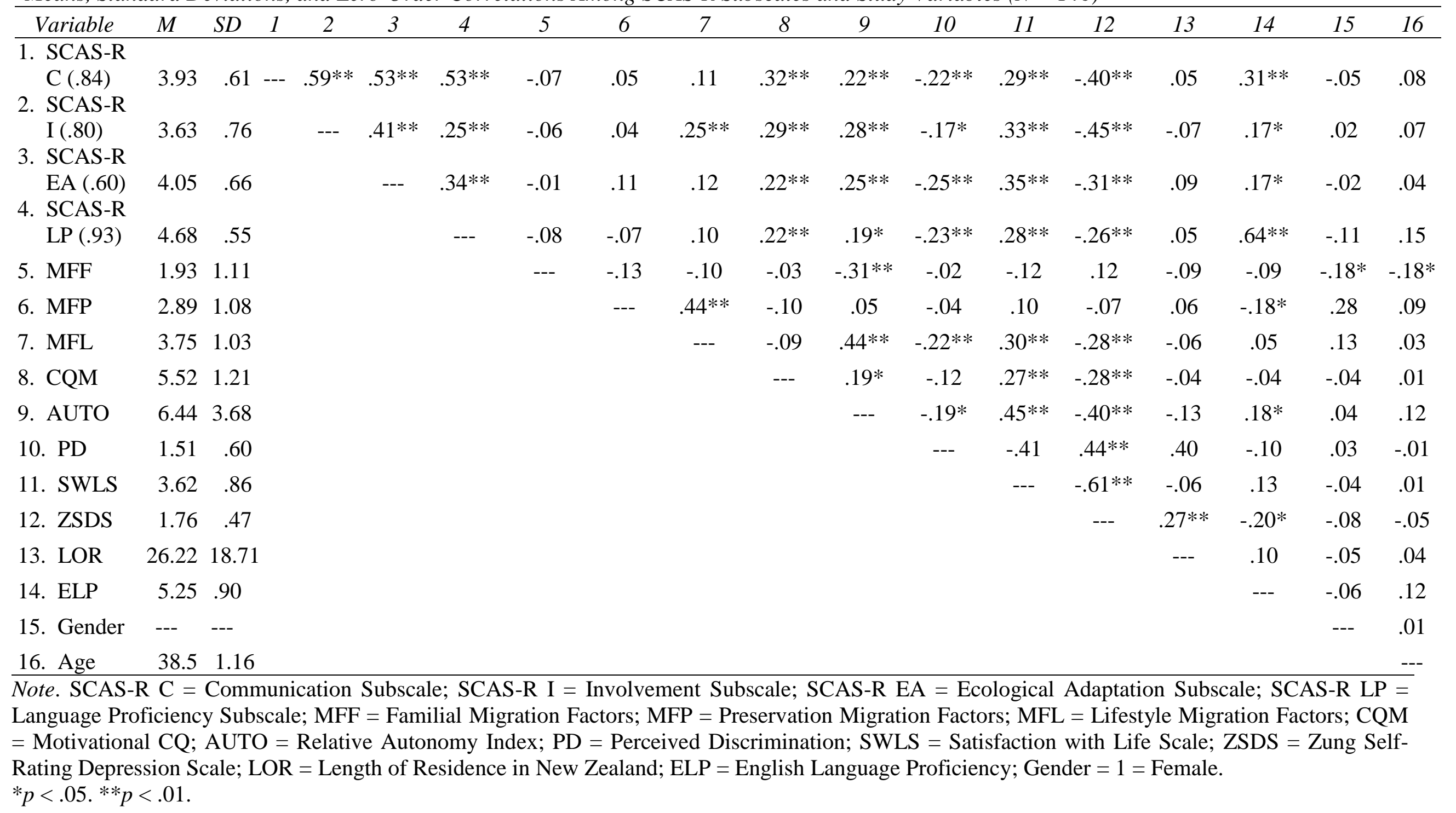




\section{Path Analyses}

Path analyses are generally the preferred method of mediation analyses for several reasons. As a straightforward extension of the single-mediator case (MacKinnon, 2000), path analyses can control for measurement error, provide detailed information of goodness of fit for the entire model, and are much more flexible than regression analyses in that multiple independent, mediator, and dependent variables may be included in the model simultaneously $\left(\right.$ Frazier, Tix, \& Barron, 2004) ${ }^{4}$. In this regard, examination of the indirect or mediated effects of sociocultural adaptation competency and perceived discrimination were possible, and a multiple-mediator model was likely to elicit a more accurate examination of these mediation effects.

As was outlined in the previous section, two hypothesised models were constructed in order to compare the indirect or mediated effects of sociocultural adaptation competency and perceived discrimination on the relationship between motivation and psychological adjustment. Data for the path analyses included 185 cases, all of which were non-missing and normally distributed, and fit the model-to-data fit criteria suggested by Bentler and Chou (1987).

Analyses were conducted in AMOS 18 (Arbuckle, 2009) using the maximum likelihood method of parameter estimation to estimate the models' parameters and goodness of fit. In accordance with AMOS procedures (Arbuckle, 2009; Byrne, 2001), overall fit for the various models was evaluated with the chi-square statistic or $\chi^{2}$, the Comparative Fit Index (CFI; Bentler, 1990), the Root Mean Square Error of Approximation (RMSEA), and the Standardised Root Mean Square Residual (SRMR). Further, to avoid difficulty ascertaining the true magnitude of any mediation effects due to the relatively small size of the sample, the path models were estimated using bootstrapping (Bollen \& Stine, 1990; Mallinckrodt et al., 2006; Shrout \& Bolger, 2002), a nonparametric approach using resampling with replacement computed multiple times (300 for the current study). The indirect effect was computed for each of the 300 samples, generating sampling distributions, bias-corrected confidence intervals, and $p$ values (Kenny, Korchmaros, \& Bolger, 2003). The

${ }^{4}$ Path analysis, a form of Structural Equation Modelling (SEM), is a procedure that allows for the investigation of relationships between one or more independent variables and one or more dependent variables (Ullman, 1996). Multiple dependent variables are often used to represent a single outcome construct, as the current study demonstrates with psychological adjustment having both positive (SWLS) and negative (ZSDS) indicators (see Hunter, 1987; Campbell \& Fiske, 1959). 
95\% confidence intervals (CI) of these mediation effect distributions were examined to determine if zero fell within the interval; if the CI excluded zero, the indirect effect was determined to be significantly different from zero at the $p<.05$ level (Shrout \& Bolger, 2002).

Migration factors path model. The first model represented a hypothesised path diagram of migration factors influencing life satisfaction and depression in New Zealand migrants. Based on empirical evidence as presented in the introduction, direct paths were anticipated between lifestyle goals, preservation goals and psychological adjustment. Further, indirect paths were anticipated from lifestyle and preservation goals through sociocultural adaptation and perceived discrimination to depression and life satisfaction. These hypotheses were generally not supported however, as the hypothesised model had poor fit, $\chi^{2}(12)=73.93, p<.001, \mathrm{CFI}=.70, \mathrm{RMSEA}=.17$ with $\mathrm{CI}_{90}(.13, .21)$, and $\mathrm{SRMR}=.10$.

A refitted model (Figure 8) was created by removing the non-significant preservation variable pathways and through correlating the life satisfaction and depression error terms. These two steps produced a significantly better fitting model $\left(\Delta \chi_{(1)}^{2}=12.60, p<.001\right) ; \chi^{2}(11)$ $=11.33, p=.42, \mathrm{CFI}=1.0, \mathrm{RMSEA}=.01$ with $\mathrm{CI}_{90}(.00, .08)$, and SRMR $=.05$. The direct and indirect effects of the refitted model can be found in Tables 11 and 12. As hypothesised, the direct effects between lifestyle goals, sociocultural adaptation, and psychological adjustment (life satisfaction and depression) were significant. These results indicate that lifestyle goals predicted greater adaptation competency, which in turn led to higher levels of life satisfaction and less depression. In regards to indirect effects, of specific interest were the significant pathways between the sequential or double mediators of sociocultural adaptation and perceived discrimination from lifestyle goals to depression $(\beta=-.07, p<.05)$ and life satisfaction $(\beta=.05, p<.05)$. Lifestyle motivation positively predicted sociocultural adaptation, which in turn predicted lower levels of perceived discrimination, which predicted better psychological outcomes (e.g., less depression and greater life satisfaction). 


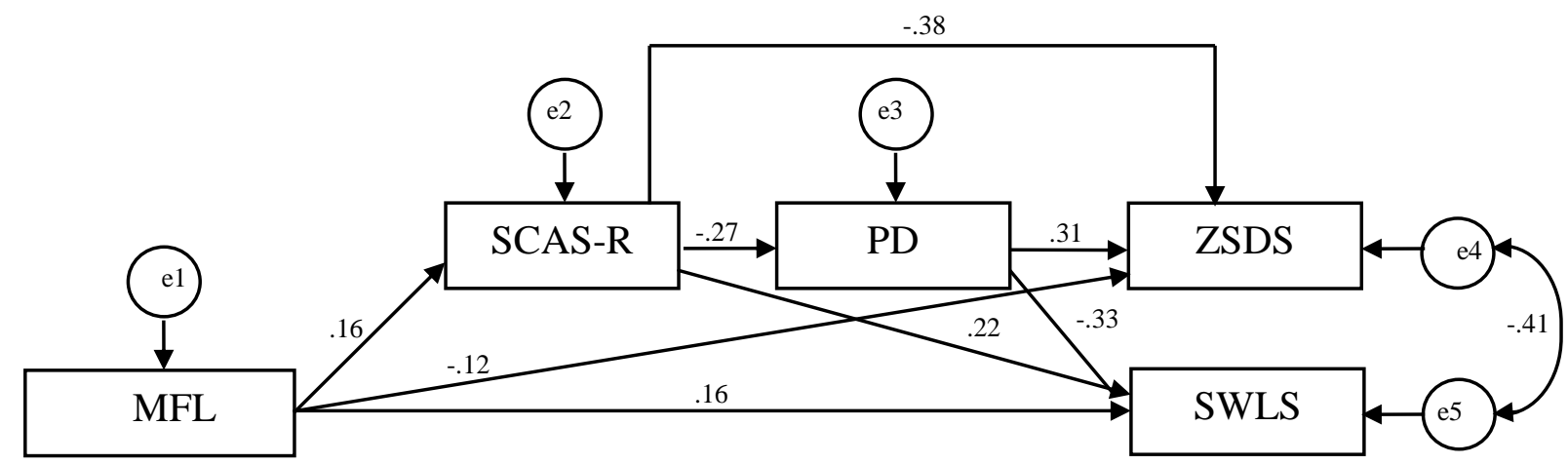

Figure 8. Refitted path model illustrating the mediated effects of sociocultural adaptation and perceived discrimination on the relationship between lifestyle goals and psychological adjustment outcomes.

Note. $\mathrm{MFL}=$ Lifestyle Migration Factors; SCAS-R = Revised Sociocultural Adaptation Scale; $\mathrm{PD}=$ Perceived Discrimination; ZSDS = Zung Self-Rating Depression Scale; SWLS = Satisfaction with Life Scale.

Table 11

Summary of the Direct Paths Between Migration Factors and Psychological Adjustment Outcomes

\begin{tabular}{|c|c|c|c|c|}
\hline Direct Effects & $\begin{array}{c}\text { Unstandardised Path } \\
\text { Coefficient (standard } \\
\text { error) }\end{array}$ & $\begin{array}{l}\text { Standardised } \\
\text { Path } \\
\text { Coefficient } \\
\end{array}$ & $95 \% \mathrm{CI}^{\mathrm{a}}$ & Sig \\
\hline $\mathrm{MFL} \rightarrow$ SCAS-R & $.08(.04)$ & .16 & $.03, .29$ & .05 \\
\hline $\mathrm{MFL} \rightarrow \mathrm{SWLS}$ & $.13(.05)$ & .16 & $.06, .27$ & .01 \\
\hline $\mathrm{MFL} \rightarrow \mathrm{ZSDS}$ & $-.05(.03)$ & -.12 & $-.22,-.03$ & .02 \\
\hline SCAS-R $\rightarrow$ PD & $-.32(.08)$ & -.27 & $-.37,-.17$ & .01 \\
\hline SCAS-R $\rightarrow$ SWLS & $.37(.12)$ & .22 & $.10, .34$ & .01 \\
\hline SCAS-R $\rightarrow$ ZSDS & $-.34(.06)$ & -.38 & $-.46,-.26$ & .01 \\
\hline $\mathrm{PD} \rightarrow \mathrm{SWLS}$ & $-.48(.10)$ & -.33 & $-.43,-.19$ & .02 \\
\hline $\mathrm{PD} \rightarrow \mathrm{ZSDS}$ & $.24(.05)$ & .31 & $.20, .40$ & .01 \\
\hline \multicolumn{5}{|c|}{$\begin{array}{l}\text { Note. MFL = Lifestyle Migration Factors; SCAS-R = Revised Sociocultural Adaptation Scale; } \\
\text { PD = Perceived Discrimination; SWLS = Satisfaction with Life Scale; ZSDS = Zung Self- } \\
\text { Rating Depression Scale; CI = Confidence Interval. a Confidence intervals are bootstrapped, } \\
\text { biased-corrected, and based on standardised path coefficients (are significant at the } .05 \text { level if } \\
\text { they exclude zero). } N=185 \text {. }\end{array}$} \\
\hline
\end{tabular}


Table 12

Summary of the Indirect Paths of Sociocultural Adaptation and Perceived Discrimination on the Relationship Between Lifestyle Goals and Psychological Adjustment Outcomes

\begin{tabular}{|c|c|c|c|c|}
\hline Indirect effects & $\begin{array}{l}\text { Standardised Path } \\
\text { Coefficient and Estimate }\end{array}$ & SE & $95 \% \mathrm{CI}^{\mathrm{a}}$ & Sig \\
\hline \multicolumn{5}{|l|}{ From MFL to PD } \\
\hline $\mathrm{MFL} \rightarrow$ SCAS-R $\rightarrow$ PD & $(.16) X(-.27)=-.04$ & .02 & $-.10,-.01$ & .02 \\
\hline \multicolumn{5}{|l|}{ From SCAS-R to Psychological Adjustment } \\
\hline $\mathrm{SCAS}-\mathrm{R} \rightarrow \mathrm{PD} \rightarrow \mathrm{SWLS}$ & $(-.27) X(-.33)=.09$ & .03 & $.04, .15$ & .01 \\
\hline $\mathrm{SCAS}-\mathrm{R} \rightarrow \mathrm{PD} \rightarrow \mathrm{ZSDS}$ & $(-.27) \times(.31)=-.08$ & .03 & $-.14,-.05$ & .00 \\
\hline \multicolumn{5}{|l|}{ From MFL to $S W L S$} \\
\hline $\mathrm{MFL} \rightarrow$ SCAS-R $\rightarrow$ SWLS & $(.16) X(.22)=.04$ & -- & -- & -- \\
\hline $\mathrm{MFL} \rightarrow \mathrm{SCAS}-\mathrm{R} \rightarrow \mathrm{PD} \rightarrow \mathrm{SWLS}$ & $(.16)(-.27)(-.33)=.01$ & -- & -- & -- \\
\hline Total Indirect Effect & .05 & .03 & $.01, .10$ & .03 \\
\hline \multicolumn{5}{|l|}{ From MFL to ZSDS } \\
\hline $\mathrm{MFL} \rightarrow \mathrm{SCAS}-\mathrm{R} \rightarrow \mathrm{ZSDS}$ & $(.16) X(-.38)=-.06$ & -- & -- & -- \\
\hline $\mathrm{MFL} \rightarrow \mathrm{SCAS}-\mathrm{R} \rightarrow \mathrm{PD} \rightarrow \mathrm{ZSDS}$ & $(.16)(-.27)(.31)=-.01$ & -- & -- & -- \\
\hline Total Indirect Effect & -.07 & .04 & $-.14,-.02$ & .03 \\
\hline
\end{tabular}

Note. MFL = Lifestyle Migration Factors; SCAS-R = Revised Sociocultural Adaptation Scale; PD = Perceived Discrimination; SWLS = Satisfaction with Life; ZSDS = Zung Self-Rating Depression Scale; CI = Confidence Interval. ${ }^{\mathrm{a} C o n f i d e n c e ~ i n t e r v a l s ~ a r e ~ b o o t s t r a p p e d, ~ b i a s e d-~}$ corrected, and based on standardised path coefficients (are significant at the .05 level if they exclude zero). $N=185$. 
Migration motivation path model. The second hypothetical model represented a path diagram of migration motivation (autonomous regulation and Motivational CQ) influencing life satisfaction and depression in New Zealand migrants. It also outlined the potential double-mediation effects of sociocultural adaptation and perceived discrimination on the relationship between migration motivation and psychological well-being. The fit indices for the hypothesised model were not acceptable, $\chi^{2}(2)=28.13, p<.001$, CFI $=.88$, RMSEA $=.27$ with $\mathrm{CI}_{90}(.19, .36)$, and SRMR $=.05$. In particular, three of the hypothesised direct paths were non-significant (AUTO $\rightarrow$ PD; CQM $\rightarrow$ ZSDS; and CQM $\rightarrow$ SWLS), indicating the absence of significant associations between autonomous regulation and perceived discrimination, Motivational CQ and depression, and Motivational CQ and life satisfaction.

Removal of these non-significant pathways significantly improved goodness of fit in the refitted model (Kline, 2005): $\Delta \chi_{(2)}^{2}=22.13, p<.01 ; \mathrm{CFI}=1.00 ; \mathrm{RMSEA}=.04$ with $\mathrm{CI}_{90}$ $(.00, .12)$, and SRMR $=.03$, leaving each of the path associations in the refitted model significant (Figure 9). This included both direct and indirect effects. In regard to direct effects, significant pathways were detected in the expected directions between autonomous regulation and the outcome variables (life satisfaction and depression). Specifically, significant direct paths were found between autonomous regulation and depression, and autonomous regulation and life satisfaction $(\beta=-.20, p=.01$ and $\beta=.32, p=.004$, respectively), suggesting that greater autonomous motivation migrants experienced in moving to New Zealand was associated with more life satisfaction and less depression. These direct paths were significant despite the presence of the sociocultural adaptation and perceived discrimination mediators. Contrary to hypotheses, however, Motivational CQ was not found to have a direct effect on either depression or life satisfaction. Estimates for all of the direct effects can be found in Table 13. 


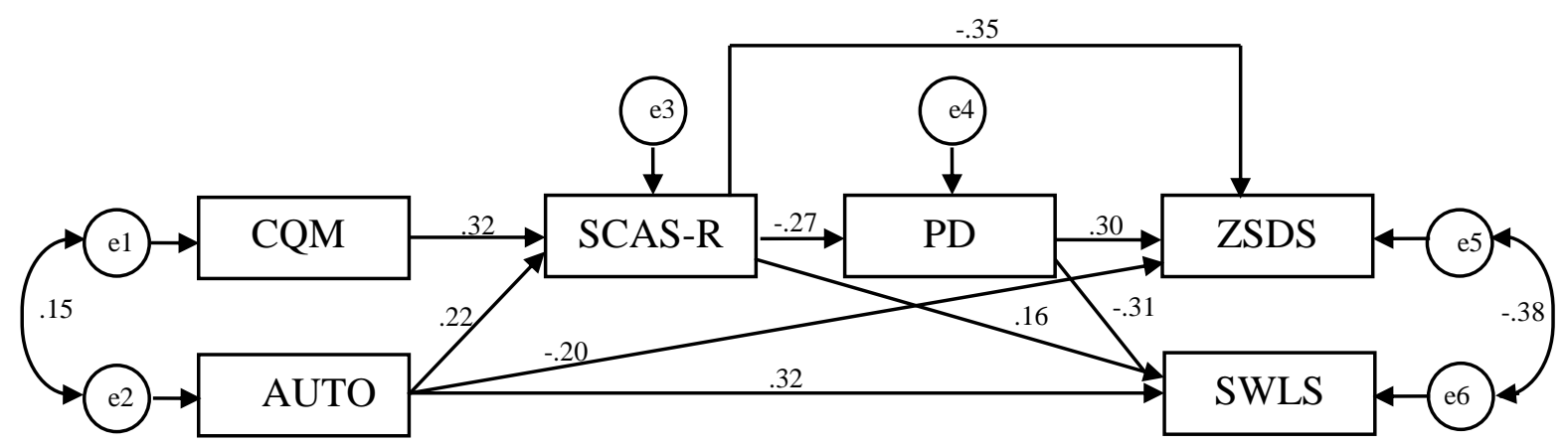

Figure 9. Refitted path model illustrating the mediated effects of sociocultural adaptation and perceived discrimination on the relationship between migration motivation and psychological adjustment.

Note. CQM = Motivational CQ; AUTO = Relative Autonomy Index; SCAS-R = Revised Sociocultural Adaptation Scale; PD = Perceived Discrimination; ZSDS = Zung SelfRating Depression Scale; SWLS = Satisfaction with Life Scale.

Table 13

Summary of the Direct Paths Between Migration Motivation and Psychological Adjustment

\begin{tabular}{lcccc}
\hline Direct Effects & $\begin{array}{c}\text { Unstandardised } \\
\text { Path Coefficient } \\
\text { (standard error) }\end{array}$ & $\begin{array}{c}\text { Standardised } \\
\text { Path } \\
\text { Coefficient }\end{array}$ & $95 \% \mathrm{CI}^{\mathrm{a}}$ & Sig \\
\hline AUTO $\rightarrow$ SCAS-R & $.03(.01)$ & .22 & $.11, .33$ & .01 \\
$\mathrm{AUTO} \rightarrow$ SWLS & $.08(.02)$ & .32 & $.21, .44$ & .00 \\
$\mathrm{AUTO} \rightarrow$ ZSDS & $-.02(.01)$ & -.20 & $-.28,-.09$ & .01 \\
$\mathrm{CQM} \rightarrow$ SCAS-R & $.13(.03)$ & .32 & $.20, .41$ & .01 \\
$\mathrm{SCAS}-\mathrm{R} \rightarrow$ PD & $-.32(.08)$ & -.27 & $-.38,-.17$ & .01 \\
$\mathrm{SCASR} \rightarrow$ SWLS & $.28(.11)$ & .17 & $.04, .25$ & .02 \\
$\mathrm{SCASR} \rightarrow$ ZSDS & $-.31(.06)$ & -.35 & $-.44,-.23$ & .01 \\
$\mathrm{PD} \rightarrow$ SWLS & $-.45(.09)$ & -.32 & $-.42,-.22$ & .01 \\
$\mathrm{PD} \rightarrow$ ZSDS & $.23(.05)$ & .30 & $.20, .41$ & .01 \\
\hline
\end{tabular}

Note. AUTO = Relative Autonomy Index; SCAS-R = Revised Sociocultural Adaptation Scale; SWLS = Satisfaction with Life $;$ ZSDS = Zung Self-Rating Depression Scale; PD = Perceived Discrimination; $\mathrm{CQM}=$ Motivational $\mathrm{CQ} ; \mathrm{CI}=$ Confidence Interval. ${ }^{\mathrm{a}}$ Confidence intervals are bootstrapped, biased-corrected, and based on standardised path coefficients (and are significant at the .05 level if they exclude zero). $N=185$.

As was previously mentioned, bootstrapping procedures were utilised in order to test the significance of the mediation or indirect effects of sociocultural adaptation and perceived discrimination within the path model. The indirect, double-mediation effects of sociocultural adaptation and perceived discrimination on the pathways between the migration motivation variables and psychological adjustment were significant. In particular, sociocultural adaptation and perceived discrimination mediated the pathway between both Motivational 
CQ and depression $(\beta=-.14, p<.01)$ and life satisfaction $(\beta=.08, p<.01)$, suggesting that individuals' confidence in their ability to function effectively in different cultural settings positively predicted sociocultural adaptation and negatively predicted perceived discrimination, which in turn predicted better psychological adjustment. Sociocultural adaptation and perceived discrimination also mediated the pathway between autonomous regulation depression $(\beta=-.10, p<.01)$, and life satisfaction $(\beta=.06, p<.01)$ : Migrants' higher levels of self-motivated interest in moving to New Zealand predicted greater adaptation competency, which in turn predicted less perceived discrimination and more positive psychological well-being. Table 14 shows the magnitude and significance for each indirect path in the model. 
Table 14

Summary of the Indirect Paths of Sociocultural Adaptation and Perceived Discrimination on the Relationship Between Migration Motivation and Psychological Adjustment

\begin{tabular}{|c|c|c|c|c|}
\hline Indirect Effects & Standardised Path Coefficient and Estimate & SE & $95 \% \mathrm{CI}^{\mathrm{a}}$ & Sig \\
\hline \multicolumn{5}{|l|}{ From AUTO to PD } \\
\hline AUTO $\rightarrow$ SCAS-R $\rightarrow$ PD & $(.22) X(-.27)=-.06$ & .02 & $-.11,-.03$ & .01 \\
\hline \multicolumn{5}{|l|}{ From CQM to PD } \\
\hline$\widetilde{\mathrm{CQM}} \rightarrow \mathrm{SCAS}-\mathrm{R} \rightarrow \mathrm{PD}$ & $(.32) \mathrm{X}(-.27)=-.09$ & .03 & $-.14,-.05$ & .00 \\
\hline \multicolumn{5}{|l|}{ From SCAS-R to Psychological Adjustment } \\
\hline SCAS-R $\rightarrow$ PD $\rightarrow$ SWLS & $(-.27) \times(-.32)=.09$ & .03 & $.05, .14$ & .00 \\
\hline SCAS-R $\rightarrow$ PD $\rightarrow$ ZSDS & $(-.27) \times(.30)=-.08$ & .03 & $-.13,-.04$ & .00 \\
\hline \multicolumn{5}{|l|}{ From AUTO to SWLS } \\
\hline AUTO $\rightarrow$ SCAS-R $\rightarrow$ SWLS & $(.22) X(.16)=.04$ & -- & -- & -- \\
\hline AUTO $\rightarrow$ SCAS-R $\rightarrow$ PD $\rightarrow$ SWLS & $(.22)(-.27)(-.31)=.02$ & -- & -- & -- \\
\hline Total Indirect Effect & .06 & .02 & $.02, .10$ & .01 \\
\hline \multicolumn{5}{|l|}{ From AUTO to ZSDS } \\
\hline AUTO $\rightarrow$ SCAS-R $\rightarrow$ ZSDS & $(.22) \mathrm{X}(-.35)=-.08$ & -- & -- & -- \\
\hline AUTO $\rightarrow$ SCAS-R $\rightarrow$ PD $\rightarrow$ ZSDS & $(.22)(-.27)(.30)=-.02$ & -- & -- & -- \\
\hline Total Indirect Effect & -.10 & .03 & $-.15,-.05$ & .01 \\
\hline \multicolumn{5}{|l|}{ From CQM to $S W L S$} \\
\hline$\widetilde{\mathrm{CQM}} \rightarrow \mathrm{SCAS}-\mathrm{R} \rightarrow \mathrm{SWLS}$ & $(.32) X(.16)=.05$ & -- & -- & -- \\
\hline $\mathrm{CQM} \rightarrow \mathrm{SCAS}-\mathrm{R} \rightarrow \mathrm{PD} \rightarrow$ SWLS & $(.32)(-.27)(-.31)=.03$ & -- & -- & -- \\
\hline Total Indirect Effect & .08 & .03 & $.04, .13$ & .01 \\
\hline \multicolumn{5}{|l|}{ From CQM to ZSDS } \\
\hline$\widetilde{\mathrm{CQM}} \rightarrow \mathrm{SCAS}-\mathrm{R} \rightarrow \mathrm{ZSDS}$ & $(.32) \times(-.35)=-.11$ & -- & -- & -- \\
\hline $\mathrm{CQM} \rightarrow \mathrm{SCAS}-\mathrm{R} \rightarrow \mathrm{PD} \rightarrow \mathrm{ZSDS}$ & $(.32)(-.27)(.30)=-.03$ & -- & -- & -- \\
\hline Total Indirect Effect & -.14 & .04 & $-.19,-.08$ & .01 \\
\hline
\end{tabular}

Note. AUTO = Relative Autonomy Index; CQM = Motivational CQ; SCAS-R = Revised Sociocultural Adaptation Scale; PD = Perceived Discrimination; SWLS = Satisfaction with Life; ZSDS = Zung Self-Rating Depression Scale; CI = Confidence Interval. ${ }^{\mathrm{a} C o n f i d e n c e}$ intervals are boostrapped, biased-corrected, and based on standardised path coefficients (are significant at the .05 level if they exclude zero). $N=185$. 


\section{Discussion}

The last study in the sociocultural adaptation research programme sought further psychometric confirmation of the SCAS-R factor structure and the scale's internal consistency. The study also tested two integrated path models that hypothesised indirect or double-mediation effects of sociocultural adaptation competency and perceived discrimination on the relationship between various components of motivation and psychological well-being. Results found mixed support for the psychometric properties of the SCAS-R: Although the scale continued to exhibit acceptable internal consistency, the refitted model generated four rather than five sociocultural adaptation factors. In regards to the hypothesised path models, the causal pathways of both were confirmed with minor modifications. Direct linkages were found between lifestyle motives and positive psychological outcomes, and behavioural competency and discrimination were found to have small but significant mediating effects on the relationship between lifestyle factors and psychological adjustment. A similar pattern was found for the path model that outlined potential relationships between migration motivation and psychological well-being.

\section{Confirmatory Factor Analysis and Internal Reliability of the SCAS-R}

Chapter 2 described development and psychometric validation of the SCAS-R in which five subscales were identified through an exploratory factor analysis: (1) Interpersonal Communication, (2) Academic/Work Performance, (3) Personal Interests and Community Involvement, (4) Ecological Adaptation, and (5) Language Proficiency. To corroborate the five-factor structure of the SCAS-R, the present study conducted a confirmatory factor analysis using a new participant sample. Contrary to expectations, the proposed structure was not replicated. Respecification of the model was required due to poor model fit, and various modifications were made based on theoretical and methodological rationale (Boomsma, 2009; Byrne, 2010). These modifications resulted in the identification of four rather than five adaptation factors: Communication (C); Involvement (I); Ecological Adaptation (EA); and Language Proficiency (LP).

Despite unsuccessful replication of the original SCAS-R factor structure, findings from the CFA support the fundamental premise that the SCAS-R captures an overall representation of cross-cultural behavioural competency as well as specific domains of behavioural adjustment. The four-factor model of the SCAS-R substantiates other research that has investigated culture change and behavioural competency as multidimensional 
constructs (see also Chapter 3). One poignant example is provided by the work of Black and colleagues (Black, Gregersen, \& Mendenhall, 1992; Black, Mendenhall, \& Oddou, 1991; Black \& Stephens, 1989), whose three-factor approach to cross-cultural adjustment includes the domains of work adjustment, adaptation to the general environment and culture, and adjustment to host national interactions. These researchers and others (e.g., Caligiuri, 2000; Parker \& McEvoy, 1993; Selmer \& Leung, 2003; Templer, Tay, \& Chandrasekar, 2006) have demonstrated both the existence and robustness of these three adjustment factors. Similarly, multiple sociocultural adaptation domains as measured by the original SCAS have also been documented. Some researchers, for example, have reported two-factor solutions involving management of behaviours and communication (Moore, 2009; Swagler \& Jome, 2005; Ward \& Kennedy, 1999), whereas others have found three-factor solutions. A factor analysis conducted by Chen (2010) outlined the following SCAS domains: Social adaptation including communicating with people from different ethnic backgrounds; physical adaptation (e.g., finding one's way around), and cultural adaptation such as understanding a host country's political system.

With this previous research on multiple adaptation domains in mind, the existence of four rather than five SCAS-R factors as outlined in the present study appears sound. Both the Language Proficiency and Communication subscales continue to reflect a fundamental tenet of culture learning theory concerning the centrality of effective communication and language skills in an individual's ability to successfully negotiate his or her new cultural setting (Argyle, 1969; Masgoret \& Ward, 2006; Ward \& Kennedy, 1999). Such language and interpersonal communication competencies are critically important for a migrant's accurate interpretation of novel social cues, and for enabling appropriate behavioural responses to norms and day-to-day routines specific to his or her host culture (Trower, Bryant, \& Argyle, 1978). Ultimately, strong verbal and non-verbal communication abilities allow a sojourning individual to develop culturally appropriate behaviours that in turn foster greater sociocultural adaptation in intercultural situations (Furnham \& Bochner, 1982). The items within these two communication and language domains adequately reflect this premise.

One unexpected result of the CFA was incorporation of various SCAS-R scale items from the original Academic/Work Performance (AWP) subscale into that of the Communication factor. Led by post-hoc analyses, a clear distinction did not emerge between various items in the original Interpersonal Communication and AWP domains. Conceptual rationale suggests this was because the AWP subscale contained several items that overlapped with the construct of interpersonal communication (e.g., "Expressing my ideas to 
other work colleagues in a culturally appropriate manner"). Results from the current study suggest that new items more specifically worded for work or academic settings would need to be generated in order to retain the AWP factor. As an example, Black and Stephen's (1989) work adjustment subscale contains items that tap a sojourner's adjustment to "Work performance standards and expectations" and "Specific work responsibilities". Overall, efforts to re-develop this domain would be advantageous, as sojourners commonly relocate for study or employment opportunities, and the ability of the SCAS-R to tap an individual's competencies or performance in these context-specific environments would strengthen the argument for the SCAS-R as a multi-faceted measure of sociocultural adaptation.

Another change made to the SCAS-R factor structure concerns development of the Involvement subscale, which highlights participative behaviours within a new host society thought to be necessary for successful adjustment. Specifically, this domain captures an individual's competence in the following areas: Developing and maintaining social networks and friendships in a novel cultural environment; becoming involved and maintaining involvement in community activities (host or ethnic); and maintaining his or her individual pursuits. A large amount of acculturation literature points to the positive intercultural adjustment benefits of involvement, such as building social networks and interacting with host nationals (e.g., Abe \& Wiseman, 1983; Clément, Baker, \& MacIntyre, 2003; Hammer, 1987). Community involvement can be heavily dependent on a migrant's linguistic selfconfidence and communication competence, as a lack of verbal skills will more than likely lead to cultural and communication misunderstandings (Marsgoret \& Ward, 2006, p. 63) or act as barriers to the development of relationships with members of the host society (Clément \& Bourhis, 1996; Noels, Pon, \& Clément, 1996). A migrant's community involvementparticularly within their own ethnic community — can also assist with maintenance of cultural and religious practices (e.g., Berry, Phinney, Sam, \& Vedder, 2006; Stuart, 2012), and research has demonstrated the importance of maintaining individual and familial pastimes and hobbies in relation to better psychological well-being and cross-cultural outcomes (e.g., Caligiuri, Hyland, Joshi, \& Bross, 1998; Mendenhall \& Oddou, 1985). For example, individuals' participation in traditional or ethnic arts activities (e.g., music, dance) has both individual and group acculturation benefits: Specifically, an individual's involvement in traditional arts activities has been found to enhance their ethnic identity and a greater sense of connectedness to their ethnic group that influences positive well-being (Fox, 2010). Ultimately, the Involvement domain of the SCAS-R captures a migrant's ability to include his or herself in the community and their competency with maintaining or developing new 
interests in the host society, all of which are central contributors to positive psychological well-being and successful sociocultural adaptation (Angel \& Angel, 2012; van Tubergen, Maas, \& Flap, 2004; Ward et al., 2010).

The Ecological Adaptation factor was maintained in the study's refitted CFA model, and, based loosely on Berry and Georgas' ecocultural framework (Berry et al., 1986; Georgas, 1993), measures a migrant's behavioural adaptation to his or her environmental surroundings. However, findings indicated low internal reliability of this SCAS-R subscale $(\alpha=.60)$, which suggests that further refinement to the domain may be necessary. For example, factor loadings of two out of three items in the domain concerning adjustment to the host society's population density and pace of life were acceptable (.58 and .84 , respectively). The third item, however, "Adapting to the noise level in my neighbourhood", had a factor loading of .33, which is below the recommended cut-off of .40 (Cronbach, 1951). Perhaps this third item is overly context-specific. As such, other items related to higher-level or more general ecocultural influences as suggested by Georgas and colleagues (Georgas, van de Vijver, \& Berry, 2004) regarding political, education, and economic systems should be generated in future validation work on the scale. Continued development of the SCAS-R and the Ecological Adaptation domain could also incorporate additional ecological frameworks, such as Bronfenbrenner's ecological systems model $(1977,1979)$ to further delineate the contextual effect of culture or society on an individual's behavioural competence.

Bronfenbrenner's model outlines four major ecological contexts or levels within which an individual interacts. The first level, the microsystem, relates to a person's immediate and proximate surroundings such as the home or workplace, whereas the second level or mesosystem encompasses the interrelationships between these various settings (e.g., interactions between workplace, family, and friends). The third ecological level, the exosystem, includes formal or informal social structures such as the government, media, and infrastructure that both directly and indirectly influence the aforementioned contexts. The last level, the macrosystem, reflects the overarching structure of a culture or society that involves both implicit ideologies (e.g., cultural values) and explicit institutional patterns (e.g., regulations or laws). Application of Bronfenbrenner's model to the SCAS-R Ecological Adaptation domain may provide a constructive way of distinguishing between the different contextual levels of a sojourning individual's new cultural environment and how his or her competencies are expressed within these various contexts.

Findings from the CFA provide continued evidence that the SCAS-R captures specific adaptation domains incorporating communication, linguistic, ecological adaptation, and 
involvement or participatory competencies known to relate to a migrant's successful crosscultural adjustment. Whether these various aspects of adjustment factor into four or five SCAS-R domains requires further resolution, however. The four-factor structure reported here and the changes seen in the original Interpersonal Communication domain may be reflections of the homogeneous participant sample that shared linguistic and cultural similarities with the host society. A five-factor model may in fact be a better fit to participant samples comprised of sojourners from more diverse backgrounds and with differing levels of host language proficiency. Additional tests of the SCAS-R model structure utilising different participant samples should be undertaken to address this issue.

\section{Pathways between Migration Factors and Adjustment}

The second objective of the current study was to test two hypothesised models of motivation and psychological well-being in relation to behavioural competency and perceived discrimination. Findings from both path models support previous studies regarding the influences of various components of migration on an individual's cross-cultural adjustment.

In regards to the first conceptual model examining push/pull relocation determinants, migrants' reports of having moved to New Zealand for pull or lifestyle reasons were directly related to greater levels of life satisfaction and fewer depressive symptoms. This was not an unexpected finding, as previous work from the areas such as sociology, tourism, and social geography has indicated that resettlement motivated by lifestyle factors-reasons such as having more leisure time, experiencing different social or cultural activities, or living in a cleaner or less crowded environment - generally leads to migrants' reports of improved wellbeing (Ewers, 2007; Legido-Quigley \& McKee, 2012; Oberoi \& Lin, 2006; Ono, 2008). What this path model adds to extant literature on acculturation, however, is a new and clearer conceptualisation of the mechanisms underpinning migrants' resettlement motives and their host country adjustment outcomes. Specifically, a migrants' lifestyle choice appears to partially influence their consequent abilities to adapt to their chosen country of residence and discrimination they may perceive: Approximately 33\% of the variance associated with lower levels of depression and around $24 \%$ of life satisfaction variance was attributed to lifestyle factors, greater behavioural competency, and less perceived discrimination. Furthermore, the direct paths of lifestyle factors and psychological adjustment were significant despite the mediation effects of behavioural competency and discrimination, suggesting that lifestyle 
motives for relocation, at least for this particular participant sample - a point that will be discussed shortly — was a powerful indicator of their adjustment to life in New Zealand.

These lifestyle or pull factors findings contrast those found for the two variables related to push motives, preservation and familial factors. Preservation migration factors (reasons for relocation due to psychological, physical, or social security) failed to significantly predict psychological well-being, despite a large breadth of literature that has reported significant relationships between push motives such as this and well-being variables including acculturative stress (Berry, Kim, Minde, \& Mok, 1987), depression (Familiar, Borges, Orozco, \& Medina-Mora, 2011; Ornelas \& Perreira, 2011), anxiety (Bauer, Priebe, Kürten, Gräf, \& Baumgartner, 1994; Teodorescu, Heir, Hauff, Wentzel-Larsen, \& Lien, 2012) and lower levels of life satisfaction (Amit \& Litwin, 2009).

Furthermore, it is of interest to note the study's results concerning the familial migration factors variable. The ability of trailing spouses or partners to contribute to or have some control in the relocation process has been associated with greater levels of satisfaction in the new host society (Hiller \& McCaig, 2007), but familial relocation can also negatively affect adjustment, particularly if one partner is more enthusiastic about the move than the other, or one partner was not a part of the decision-making process to migrate (Tabor \& Milfont, 2012). No hypotheses for familial migration factors were formed due to the contradictory and scant nature of such existing literature. However, zero-order correlations found that familial migration factors were unrelated to all of the mediation and outcome variables. These non-significant findings may be in part due to the low reliability of this particular migration factor subscale $(\alpha=.60)$ : This is the first known modification of items from the Department of Labour's Settlement Report (IMSED, 2008) concerning specific factors that may have motivated participants' moves to New Zealand, and as such may be prone to potential psychometric shortcomings. With this in mind, future studies utilising this instrument to capture migration factors will benefit from close examination of factor analyses derived from these various items. Family relocation is undoubtedly an important motive for international relocations and as such deserves further empirical scrutiny.

A plausible explanation for the differing results found between these pull and push motives and cross-cultural adjustment may lie with the over-representation of Western, welleducated, and native English speakers in the present study's participant sample. For example, lifestyle migration (e.g., pull factors) appears to occur more amongst members of relatively affluent and industrialised countries who seek more relaxed or meaningful lives abroad (see Benson \& O'Reilly, 2009). In fact, some researchers suggest that lifestyle 
migration is a product of Western individualism, typifying the individual pursuit of happiness (cf., Benson, 2011; Giddens, 1991) and that those who move abroad do so as an act of selfdiscovery (Korpela, 2011). Similarly, the study's findings regarding lifestyle factors may also reflect a specific type of "migrant personality" (Boneva \& Frieze, 2001) in the participant sample. It may be that people who are more extraverted, curious about different cultures, open-minded, flexible, or who enjoy the challenge of experiencing new cultures and improving their communication or cultural competencies may be more likely to migrate than others without these characteristics (Boneva \& Frieze, 2001; Richardson \& Rullo, 1992). Ultimately, push-pull factors that motivate a migrant's international relocation are dependent upon contextual circumstances as well as individual differences (Martin, 1993): Examination of how these personal and contextual factors interact to form the relocation motivations of migrants and how these motivations in turn influence their consequent adjustment to their host societies warrants further empirical scrutiny.

\section{Pathways between Migration Motivation and Adjustment}

A review of studies on migration motivation, competence, discrimination, and psychological well-being pointed to evidence for independent associations between some of these variables; however, the combined study of all four is a new research endeavour. As such, the second hypothesised model examined the potential mediation effects of sociocultural adaptation competency and perceived discrimination on the relationship between migration motivation and psychological adjustment.

A main finding from the second path model suggests that the relationship between Motivational CQ and psychological adjustment is fully mediated by sociocultural competency and perceived discrimination. Work embracing the cultural intelligence framework has found it to predict performance-related adjustment within social, personal, and work domains, and also psychological indicators such as life satisfaction and less depression (Ang et al., 2007; Early, 2002; Templer, Tay, \& Chandrasekar; 2006; Van Dyne et al., 2012), with Motivational CQ sometimes offering greater explanatory power in the prediction of these outcomes than the other CQ components (Ward, Fischer, Lam, \& Hall, 2008; Ward, Wilson, \& Fischer, 2011).

Both direct and indirect effects were discovered between the other motivation migration component, autonomous regulation, and the psychological well-being outcomes, suggesting a partial mediation effect for the competency and discrimination variables. This is not a surprising finding, as items from the Autonomy Index emphasise the psychological 
processes behind a migrant's decision to move abroad, and as such evoke more emotivebased responses around items concerning moves made out of shame, guilt, pride, personal values, etc. Accordingly, the Autonomy Index's emphasis on affective drivers of relocation have a stronger association with the psychological indicators of life satisfaction and depression that have also been noted in previous acculturation studies (Chirkov et al., 2008; Chirkov et al., 2007; Vansteenkiste et al., 2006). Overall, the topic of migration motivation warrants continued examination, particularly with regard to further delineation of how specific cross-cultural motivation components influence an individual's adjustment to a novel cultural milieu and the differing pathways that may exist between these constructs.

\section{Pathways between Perceived Discrimination and Psychological Adjustment}

A large amount of literature has examined the effects of perceived discrimination on psychological well-being, including various meta-analyses (Lee \& Ahn, 2012; Pascoe \& Smart, 2009) and work involving acculturating persons (e.g., Neto \& Barros, 2000; Vedder, Sam, \& Liebkind, 2007; Ward, Bochner, \& Furnham, 2001). These studies and others including the ICSEY project (Berry, Phinney, Sam \& Vedder, 2006) have provided strong evidence for the negative influence of various forms of discrimination on the psychological health of ethnic minorities and migrant populations including increased anxiety, depression, acculturative stress, and anxiety. Results of the present study reflect similar relationships, with correlations and direct pathways indicating significant associations between perceived discrimination and psychological health and adaptation. Although discrimination can be both subtle and overt, the present study measured general and relatively subtle forms of perceived discriminatory behaviours in relation to life satisfaction and depression. In order to better understand the complexities surrounding discriminatory experiences and their effect on migrants' well-being, future research would benefit from including measures that examine subtle as well as more blatant forms of discrimination and/or frequency of perceived discriminatory experiences.

\section{Pathways between Behavioural Competency, Perceived Discrimination, and Psychological Adjustment}

Direct pathways were discovered between sociocultural competency and perceived discrimination as well as between competency and the two psychological adjustment indicators in both hypothesised models. Various studies support these pathways. Work with Korean women in the United States by Yoon and colleagues, for example, has found evidence for the roles communication and linguistic competencies play in decreasing the 
perceived effects of discrimination (Yoon, Lee, Koo, \& Yoo, 2009; Yoon et al., 2012). In their qualitative study (Yoon, Lee, Koo, \& Yoo, 2009), participants reported better language and communication competency over time that in turn assisted them in challenging discriminatory behaviours directed against them. Further, decrements in culturally appropriate skills (e.g., interpersonal competence), host language fluency, and behavioural problems in minority adolescents have also been associated with increased perceptions of maltreatment (Neto, 2006; Phinney, Madden, \& Santos, 1998; Oppedal, Røysamb, \& Sam, 2004) as well as actual discriminatory experiences (García Coll et al., 1996; Kim, Wang, Deng, Alvarez, \& Li, 2011). In sum, difficulty in attaining culturally appropriate skills and interacting with host nationals and unfamiliarity with host culture norms can be seen to contribute to a migrant's heightened experience of perceived discrimination.

The positive influence of cultural competence on psychological adjustment has also been documented. One study investigating intercultural communication competency in a sample of international students divided the construct into components such as communication skills, language competence, communication effectiveness, and social integration (Redmond \& Bunyi, 1993), finding that several of these competency components predicted how the students experienced and handled stress. Additionally, in interviews with groups of international students attending various universities in New Zealand, Lewthwaite (1996) reported that communication mishaps and inabilities to establish interpersonal relationships led to students' higher levels of stress as well as lower degrees of adaptation.

\section{Indirect Pathways: The Mediating Effects of Competency and Perceived Discrimination}

Although the pathways between behavioural competency, less perceived discrimination, and greater psychological adjustment have received scant attention, some support for this relationship can be found in studies regarding stigma and resilience, competency-related skills, and training interventions. Several studies regarding destigmatisation strategies, for example, have evidenced the benefits of teaching social skills competency, visualisation of positive intergroup encounters, increasing language skills, and active community involvement in order to diminish individuals' perceived sense of stigma that have consequently improved positive psychological outcomes (Ilic et al., 2012; Stathi, Tsantila, \& Crisp, 2012). Further, emotion recognition, social interaction, verbal and nonverbal communication, and role-play training have been found to diminish self-perceived stigma and raise self-esteem in individuals with socially contractible illnesses (Augustine, Longmore, Ebenezer, \& Richard, 2012). Intervention strategies targeted at individuals with 
mental illnesses who have faced stigmas against them have also included programmes such as one led by Lucksted (Lucksted et al., 2011) on cognitive reframing and learning new behavioural responses to stigma and discrimination. This particular programme was found to decrease perceived stigma after 9 group sessions, and to increase individuals' sense of empowerment and other positive psychological indices. These studies, along with the present research, help to elucidate the role intercultural behavioural skills training may play in reducing migrants' potential experiences of discrimination as a route to better psychological adjustment within their host societies.

Last, indirect effects between motivation, cultural competency, discrimination, and psychological well-being were discovered. The degree of sociocultural competence and discrimination migrants perceived was found to mediate the association among various components of their motivations to relocate, their life satisfaction, and reported depression. In the first hypothesised model, partial mediation effects of sociocultural competency and perceived discrimination were discovered between lifestyle migration factors and psychological well-being. These results suggest that migrants who moved to New Zealand for lifestyle reasons report greater life satisfaction and less depression, but that the degree of behavioural competency they have within their new host environments and their perceptions of discriminatory actions against them also have an influence on their psychological wellbeing. The indirect pathways found within the lifestyle motivation factor path model begin to address the mechanisms through which this relocation motive can affect well-being in a migrant's new host country through highlighting the important roles competency and perceived discrimination may play in governing the association between these variables. These potential pathways to successful cross-cultural adjustment are particularly relevant to New Zealand and other countries with large numbers of globally mobile, voluntary migrants (IMSED, 2008).

The second path model suggests that the relationship between a migrants' migration motivation and resultant psychological well-being may operate through his or her level of behavioural competency and experience of discrimination. These findings advance the possibility that greater cross-cultural confidence and intrinsic migration motivation, in combination with proficient sociocultural competencies an individual exercises within a new cultural environment, can facilitate coping strategies and bolster resiliency against potential experiences of discrimination encountered during the acculturation process. 


\section{Limitations and Future Directions}

Findings from the present study should be considered in light of several limitations. First, further validation of the SCAS-R factor structure is required, as the hypothesised fivefactor model was not replicated in the present study and explorative modification procedures were utilised. Additional reliability and validity studies of the SCAS-R are also needed, particularly research concerning divergent validity, in order to examine how distinct the SCAS-R is from other cross-cultural adjustment measures. Cross-validation work with the SCAS-R using different and more representative participant samples should also be considered. The importance of further validation work cannot be emphasised enough, as "an ounce of replication is worth a ton of inferential statistics" (Steiger, 1990, p. 176 as cited in Boomsma, 2009).

Second, path model results should be interpreted with some caution due to various methodological issues. Self-report measures of perceived discrimination, for example, are dependent upon an individual's ability to accurately identify discriminatory cues. Many migrants entering new and perhaps culturally-distant host societies would find interpretation of various actions towards them challenging, particularly non-overt or subtle forms of prejudice. In addition, both of the migration motivation components were measured retrospectively after relocation had occurred. Retrospective or post-migration accounts are not as accurate as pre-migration responses would be (Winchie \& Carment, 1989), and given the relatively few number of studies that have focussed on migrants prior to relocation, further work considering pre-move migrants is recommended.

Furthermore, as a cross-sectional study, the analyses presented here are intrinsically limited in their ability to infer causal relationships. Although the utilisation of extant theories, previous empirical findings, and cross-sectional data helped to elucidate possible associations, no empirical certainties concerning causality can be provided (Kraemer, Yesavage, Taylor, \& Kupfer, 2000). Experimental designs or longitudinal studiesespecially those measuring migration factors and migration motivation before departure-are needed in order to provide a more precise and empirically sound conceptualisation of the mediating roles of sociocultural adaptation competency and perceived discrimination in the relationship between motivation and psychological adjustment. Continued structural equation modelling research on this topic would also benefit from testing alternative models (e.g., discrimination as a predictor of sociocultural competency, different mediators) to provide further evidence for or to refute the mediation effects proposed here. 
Another limitation of the study's findings involves the participant sample. As has been mentioned previously, the majority of individuals who volunteered their time for the study were highly educated, from culturally-similar countries to New Zealand such as the United Kingdom, and either spoke English as a first language or reported extremely high levels of English language fluency. Further, the overrepresentation of women in the sample may have also skewed findings regarding the migration-adjustment outcomes experience. For example, gender-related roles, expectations around these roles, and gender itself are all extremely influential factors in the structural composition of both society and the family, and these factors affect migration processes as well (cf., Donato et al., 2006). Research on migrant populations is ideally conducted on a sample representative of the population in order to best generalise findings.

Despite these limitations, the current research added to literature on culture contact through (1) continued exploration of the conceptual and psychometric underpinnings of the SCAS-R, and (2) investigation of migrants' motivations for moving to New Zealand and their psychological well-being in relation to behavioural competency and perceived discrimination. The competence-based approach afforded by the SCAS-R in this study allows for an account of how migrants may utilise the behavioural skills they have acquired in their new host environments to approach difficult discriminatory situations, which can then affect their psychological responses and positively contribute to general well-being. Further discussion of the contributions this and the previous two studies in the sociocultural adaptation research programme make to the acculturation field will be addressed in the next chapter, along with consideration of how this collective body of research may be applied in other theoretical, empirical, and training settings. 


\section{Chapter Five: General Discussion}

This research programme investigated sociocultural adaptation, a behavioural aspect of cross-cultural competency that is situated within the culture learning framework of acculturation psychology and involves an individual's ability to acquire culturally appropriate skills and negotiate new cultural settings. Through the review, revision, and expansion of the acculturation literature on sociocultural adaptation, this research programme has offered new insights into the factors that assist and hinder the acculturation process, and has examined how culturally-based behavioural competence is defined and measured within the acculturation psychology domain. The first study, a meta-analytic review, considered the demographic, situational, individual differences and psychological correlates of sociocultural adaptation as measured by the Sociocultural Adaptation Scale or SCAS (Searle \& Ward, 1990). Results of the meta-analysis emphasised the importance of individual differences (e.g., personality, motivation) and discrimination in relation to sociocultural adaptation difficulties. The second study reframed and revised the SCAS, and explored specific interpersonal, involvement, work/academic, ecological adaptation, and language domains of behavioural adjustment. The third and concluding study continued investigation of the psychometric and conceptual underpinnings of the revised SCAS, and utilised findings from the meta-analysis on motivation and perceived discrimination to examine the possible causal links between these variables and cross-cultural adjustment. The following chapter will: (1) Consider how the findings from the studies presented within this research programme contribute to the acculturation literature and other disciplines; (2) outline the limitations of the thesis, and (3) suggest how researchers and practitioners can utilise the new information on sociocultural adaptation provided here to assist with migrants' successful and effective cross-cultural adjustment.

\section{Key Findings and Implications}

\section{Beyond Culture Learning Theory: A New Look at Sociocultural Adaptation Correlates}

Research within the culture learning framework of acculturation has traditionally focussed on variables associated with the learning process. As such, a limited amount of conceptual and empirical work has considered how individual differences such as personality and motivational factors affect an individual's adjustment to new sociocultural settings. Drawing upon existing personality and motivation theories, Studies 1 and 3 of the current research programme addressed this gap in the culture learning literature by providing 
quantitative evidence that individual differences are important aspects of an individual's successful intercultural adjustment.

Personality. Many researchers agree that the lack of sound theoretical frameworks involving personality and cross-cultural competency has impeded theoretical and empirical development in the acculturation field (Berry, Poortinga, Breugelmans, Chasiotis, \& Sam, 2011; Kosic, 2006; Ward, Leong, \& Low, 2004). This thesis has highlighted two specific theories that may be beneficial in advancing the study of individual differences within an acculturative processes paradigm. The ecocultural framework (Berry, 2001, Berry et al., 1986; Georgas, 1988, 1993; van de Vijver, \& Berry, 2004) utilised throughout the thesis posits that personality influences behaviour, and that both personality and behaviour are influenced by contextual (e.g., socio-political, ecological) contexts. Mischel and Shoda's (1995) cognitive-affective theory of personality is also recommended as a foundation for continued personality and acculturation research. This framework proposes that an individual's personality can be seen as a relatively predictable system that guides and shapes behaviours. These two personality theories may be utilised within the intercultural field as platforms upon which to build future acculturation work. For example, long-standing questions related to the superiority of some personality traits over others in predicting effective cultural competency and adjustment could be framed within these paradigms. The ecocultural and cognitive-affective theories could also be referenced in order to compare and contrast how personality traits are negotiated and expressed within different cross-cultural situations, or how these traits affect behavioural skill acquisition within novel environments.

Along with presentation of these personality frameworks, the research programme also provided empirical support for the view that various personality components influence effective cross-cultural adjustment. Meta-analytic results in Study 1 illustrated significant effects between broad personality factors such as the Big Five, more narrowly defined culture-centred aspects of personality such as cross-cultural self-efficacy and cultural empathy, and sociocultural adaptation. Personality measures are often utilised in the selection of international personnel (Bernardin \& Bownas, 1985; Deller, 1997; Ones \& Viswesvaran, 1999) and in the investigation of how various personality components relate to different acculturative outcomes (Aycan, 2008; Ali, Van der Zee, \& Sanders, 2003; Kealey, 1989; Padilla, Wagatsuma, \& Lindholm, 1985). Findings from the current research provide empirical justification for continued inquiry into the nature of these personality-performance and personality-adjustment relationships. 
The present work also highlights the importance of measurement specificity with regard to personality measures and culture learning outcomes. In Study 1, culture-centred personality factors (e.g., cultural empathy) were more strongly associated with sociocultural adaptation than measures capturing general personality traits. Other research investigating the operationalisation of personality factors has corroborated this finding that contextualised personality measures are stronger predictors of performance indicators than noncontextualised instruments (Shaffer \& Postlethwaite, 2012). Van Dyne and colleagues have provided further commentary on the issue, writing that specific conceptualisations of a construct allow "for more refined theorising and testing" (Van Dyne et al., 2012, p. 296).

These results highlighting the importance of measurement specificity have several applications. For the international business, management, and organisational fields, incorporation of culture-centred personality instruments into recruitment and selection processes could allow for more accurate predictions of an indiviudal's potential success on an international assignment or, conversely, enhanced forecasting of premature assignment terminations. Such instruments could also provide the aforementioned fields with the capability to better predict a candidate's ability to work with or manage culturally-diverse teams or employees. In a clinical context, culture-centred personality instruments could be used to better inform treatment plans, counselling sessions, or other services provided to sojourning individuals (e.g., international students who may be experiencing acculturation difficulties). Last, use of culture-centred personality instruments within the acculturation field would augment our understanding of the predictive influences of personality factors on an individual's cultural skill acquisition and intercultural adjustment to a new host society.

\section{Migration Factors and Migration Motivation}

Examination of what motivates an individual's relocation to a different country is an underrepresented research area, particularly within the acculturation and culture learning literature (Gezentsvey \& Ward, 2008; Kosic, 2004; Padilla \& Perez, 2003; Winchie \& Carment, 1989). Meta-analytic findings in Study 1 substantiated recommendations from these academics and others for the continued investigation of motivation and intercultural adjustment: Cross-cultural self-efficacy and other motivation-related components were strongly correlated with sociocultural adaptation despite the small number of studies included in the meta-analysis examining these associations. Further, based on results from the metaanalysis, Study 3 examined a range of push-pull migration motivation components and their respective effects on cross-cultural adjustment. Such research provides the acculturation and 
related fields with a more thorough understanding of the determinants of migration motivation from a distinctly psychological perspective. The research programme's findings on migration motivation may also act as a foundation for how motivation theories such as self-determination theory (SDT) can be integrated with culture contact research. Last, findings from the research programme may be utilised as an empirical platform for intercultural trainers or training providers interested in developing and implementing training programmes that incorporate motivation and/or cross-cultural behavioural competency components.

Areas such as geography, sociology, and particularly economics have been the prominent leaders in the examination of push-pull migration determinants (Chirkov et al., 2007; Martin, 1993). However, the vast majority of work done in these fields has concentrated on labour migration or the economic factors that influence an individual's pursuit of employment overseas (Goss \& Lindquist, 1995). Indeed, some researchers have remarked that migration research often views migrating individuals as "mere clotheslines on which to hang propositions of economic logic" (Schumpteter, 1954; pp. 885-886 as cited in Portes, 1997).

The current work contributes a psychological perspective and adds to the acculturative literature on migration research by highlighting how migration motivation determinants such as lifestyle migration factors, intrinsic migration motivation (e.g., autonomous regulation), and Motivational CQ (a factor of cultural intelligence) positively influence cross-cultural adjustment. These components, representative of contextual or situation-specific factors as well as individual psychological differences, were found to be significant indicators of migrants' higher sociocultural competency, less perceived discrimination, and more positive psychological well-being. As such, this research goes some way in elucidating the effects of non-economic drivers of relocation on successful intercultural adjustment outcomes.

A substantial amount of literature has utilised the cultural intelligence framework to consider the specific role Motivational CQ plays in both effective behavioural competencies and positive well-being outcomes. However, much less work has explored how the other motivation theory employed in this research programme, Self-Determination Theory or SDT, can be integrated into the culture learning and acculturative paradigms. Self-Determination Theory postulates that behaviours derived from an individual's inherent values and interests (e.g., intrinsic motivation) engender better performance outcomes when compared to behaviours controlled or regulated by the extrinsic values and interests of others (Ryan \& 
Deci, 2000). Although SDT has received extensive attention in various areas of social psychology, education, management, and health care, it has only recently been applied to the phenomena of migration motivation and cross-cultural adjustment. The SDT framework offers a sound empirical and theoretical approach to the study of intrinsic and extrinsic motivation, and findings from this research programme suggest it would be a useful conceptual tool for further incorporation into the field of acculturation psychology.

This research programme may also be used as a foundation for intercultural training programmes that involve motivation components. There are three broad categories of intercultural effectiveness that were mentioned in the first chapter: (a) A cognitive aspect regarding knowledge and perceptions about a different culture; (b) a skill component concerning behaviours acquired in new settings; and (c) an intrapersonal element involving attitudes and motivations towards novel situations (Hannigan, 1990). Comprehensive intercultural training courses should involve incorporation of all three of these capacities in order to best develop an individual's effective cultural competency. Most programmes already entail some form of skills training that can be seen to fall under the culture learning framework, such as identification of cross-cultural differences and minimisation of these differences through skills streaming, simulations, modelling, feedback, or role playing (Brislin \& Yoshida, 1994). The current work suggests that training within the third rubric of intercultural effectiveness could also be presented through concepts derived from Motivational CQ and SDT regarding intrinsic motivation. First, individuals could be provided with ways to develop awareness of the intrinsic or extrinsic motives influencing their decisions to move abroad ${ }^{5}$. In addition to engendering developing awareness around intrinsic/extrinsic migration motivation, training procedures could be generated to increase participants' intrinsic motivation towards living abroad. These procedures could involve activities that encourage participants to think about the intrinsically interesting or enjoyable aspects of living in a new country, or ways in which individuals can make routine international assignments more stimulating.

\footnotetext{
${ }^{5}$ The Conscious/Competent Developmental Model (Howell, 1986; Howell \& Fleishman, 1982) could provide a useful training framework for increasing an individual's awareness of both migration motives and cross-cultural competencies. This cross-cultural competence model posits that an individual progresses through increasing stages of awareness, from being entirely unaware of their affective, behavioural, and cognitive intercultural competencies or weaknesses to more advanced stages of competence development where conscious thought about actions or performance is not necessary.
} 


\section{A Revised Measure of Sociocultural Adaptation}

A central objective of the research programme was to examine how sociocultural adaptation is operationalised as a behavioural facet of cultural competency within the acculturation framework. Study 2 attended to this topic through investigation of existing issues with the original SCAS and development of a revised measure. Further, both Studies 2 and 3 examined specific adaptation domains, including the domain of host country language proficiency.

Improved operationalisation. A major concern in acculturation psychology has involved the conceptualisation and measurement of cross-cultural adjustment, with undifferentiated use of terms such as assimilation, cross-cultural effectiveness, culture shock, adaptation, and acculturation impeding more rigorous empirical inquiry. To address this issue, a central aim of the present work involved development of a revised measure of the SCAS. The SCAS-R refined sociocultural adaptation as a measure of behavioural adjustment through the use of new terminology concerning an individual's newly-acquired competencies within a novel cultural environment. The valence of the original instrument was also changed: Whereas the original scale captured degree of intercultural difficultymaladjustment, in essence-the revised instrument reflects an individual's degree of intercultural behavioural competency. In these ways, it is posited that the SCAS-R provides a more concise understanding of an individual's adaptation and settlement within a new country. The revised instrument engenders a more unequivocal positioning of the sociocultural adaptation construct within the culture learning framework, and as such aims to improve the quality of future empirical work within the field of acculturation research.

Multiple adaptation domains. The revised sociocultural adaptation scale measures an overall representation of adaptation as well as specific adaptation domains. The theoretical underpinnings of these domains include aspects of culture and social learning theory (Black \& Mendenhall, 1990; Bochner, 1972; Furnham \& Bochner, 1986), the abilitybased model of emotional intelligence (Mayer \& Salovey, 1993), the cultural intelligence paradigm (Ang et al., 2007), adaptive performance (Pulakos et al., 2000), and the ecocultural framework (Berry et al., 1986; Georgas, 1988). The versatility of these SCAS-R domains should prove useful to future research and training endeavours in a variety of ways. For example, the ability to capture various competency domains will enable acculturation researchers to determine what specific areas of adaptation may prove more challenging than others for sojourning individuals or migrant groups. The multi-factor structure of the SCAS$\mathrm{R}$ would also allow for investigation of the predictive relationships between different 
antecedents and sociocultural adaptation domains to add a more comprehensive understanding of how behavioural competency differs between individuals. Domain constellations could also be created to form a detailed behavioural profile of an individual, which would be beneficial in applied settings for testing the efficacy of intercultural training programmes or pinpointing competency areas requiring further development.

Centrality of language proficiency. The centrality of language proficiency to the sociocultural adaptation construct has been established throughout the research programme. The introductory chapter of this thesis reviewed theoretical rationale concerning the centrality of communication and language skills to the culture learning framework. Language proficiency facilitates communication of cultural information, and the absence of host language ability can hinder development of meaningful relationships with host nationals or encumber the completion of day-to-day tasks in a foreign environment (Church, 1982; Marsgoret \& Ward, 2006; Noels, Pon, \& Clément, 1996). The meta-analysis outlined in Chapter 2 found that language proficiency was associated with successful adjustment outcomes. Studies 2 and 3 revealed a specific language competency domain embedded within the sociocultural adaptation construct, which corresponds with other researchers' conceptualisations (Berry, Phinney, Sam, \& Vedder, 2006; Masgoret \& Ward, 2006) of foreign language proficiency and communication competence as central components of successful social interaction and key indicators of acculturation. In an applied sense, the centrality of language proficiency as demonstrated in the present work suggests that international student offices and pre-departure training programmes should continue a heavy emphasis on language training to individuals.

\section{New Models of Cross-Cultural Adjustment}

A novel approach to sociocultural adaptation was presented in Study 3 through the empirical examination of two hypothetical path models involving migration motivation, discrimination, and cross-cultural adjustment. These models are innovative to the acculturation literature in two key ways. First, most research on these topics has only examined the independent associations between them, whereas Study 3 focussed on the potentially causal associations between these variables. Study 3 also considered the doublemediation effects of sociocultural adaptation competency and perceived discrimination, which were found to influence the relationship between aspects of motivation and psychological well-being. The findings from this last study have conceptual as well as methodological implications for the culture contact field. 
Conceptual considerations. Findings from Study 3 offer the acculturation psychology paradigm a novel conceptual approach to the relationships between the affective, behavioural, and cognitive components of culture change. As is illustrated in Figure 10, an individual's perception of discrimination - a cognitive facet of social identification theorywas found in Study 3 to mediate the association between behavioural competency, an aspect of culture learning, and psychological or affective outcomes (variables related to the stress and coping framework). The configuration of culture learning as an antecedent to these cognitive and affective components of acculturation is an innovative conceptualisation to the acculturation literature, as the majority of work in the field positions behavioural competency as a long-term adaptive outcome or end result of an individual's intercultural experience.

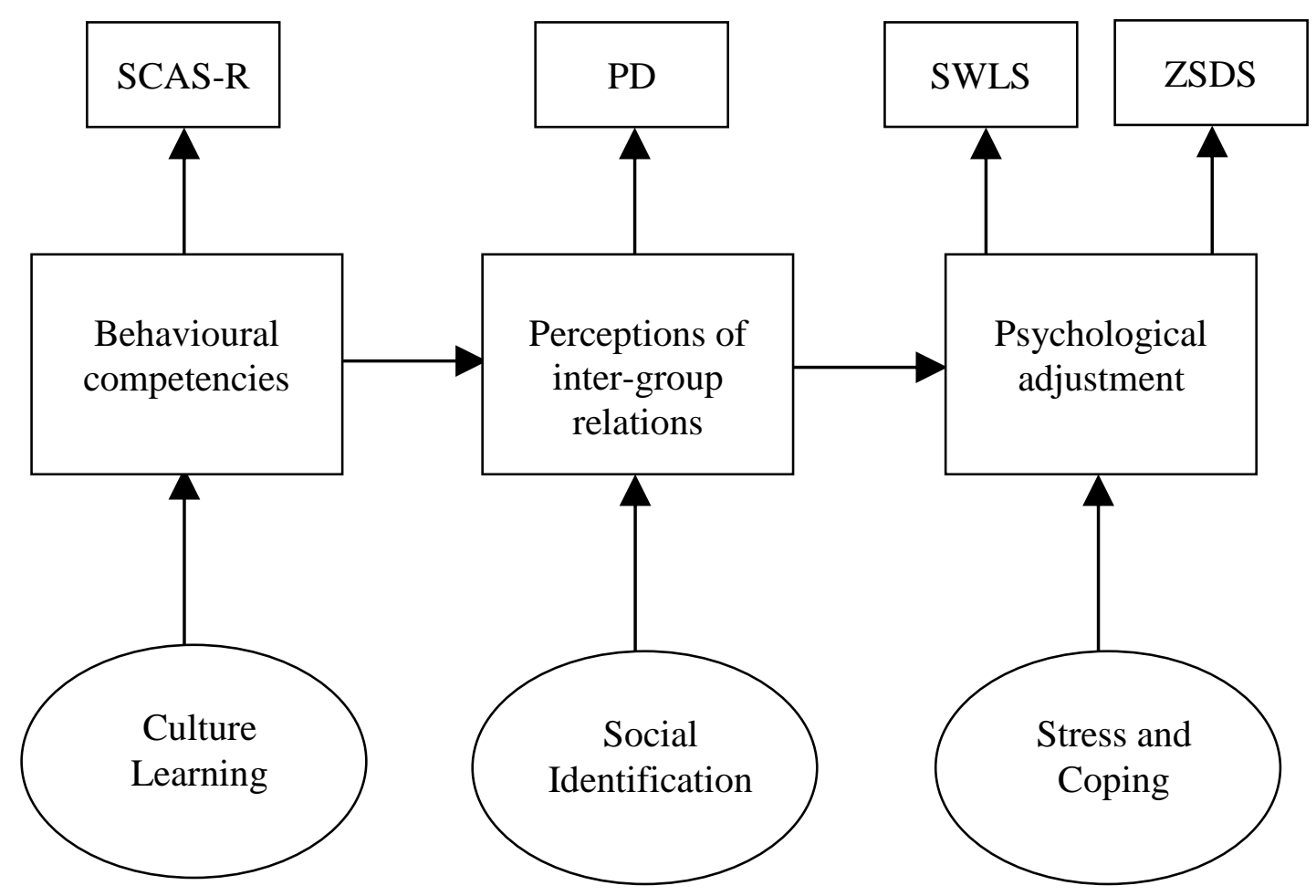

Figure 10. Hypothesised pathways tested in Study 3 between the frameworks, processes, and outcomes of the ABC model of culture contact.

Note. SCAS-R = Revised Sociocultural Adaptation Scale; $\mathrm{PD}=$ Perceived Discrimination; SWLS = Satisfaction With Life Scale; ZSDS = Zung Self-Rating Depression Scale.

Methodological considerations. Study 3 provides a more in-depth understanding of how a migrant's successful psychological adjustment may be influenced by motives that led to their relocation, their ability to learn and enact behaviours that are culturally appropriate within their host society, and their perceptions of discriminatory actions against them. The 
path analyses undertaken in Study 3 allow more insight into the associations between these variables than would be possible through other mediation methods for several reasons (e.g., simultaneous examination of all variables, the ability to control for measurement error, inclusion of multiple antecedent, mediator, and outcome variables; see Chapter 4 for further information). Based on previous theoretical and empirical findings, the models described in this research programme contribute to acculturation research through more accurate examination of the potential mediation effects of sociocultural competency and discrimination.

In sum, the competence-based approach taken within the path models allows for a greater understanding of how migrants may utilise the behavioural skills they have acquired in their new host environments to approach difficult discriminatory situations, which can then affect their psychological responses and positively contribute to general well-being. These findings advance the possibility that greater cross-cultural confidence and intrinsic migration motivation, in combination with proficient sociocultural competencies an individual exercises within a new cultural environment, can facilitate coping strategies and bolster resiliency against potential experiences of discrimination encountered during the acculturation process.

\section{Refining the Model and Measurement of Acculturation}

Operationalisation of the term "acculturation" remains an elusive challenge within the social sciences that "has, no doubt, limited the scientific exchange of information and meaningful discussion around research findings and theory development” (Sam, 2006, p. 11). The work presented in this thesis has endeavoured to address this challenge in the acculturation literature through focussing on the measurement and conceptualisation of sociocultural adaptation.

As a measurement of sociocultural adaptation, the SCAS has been widely utilised both within acculturation research and across other fields including international business and management, communication and language acquisition, education, and organisational psychology (Kim, 2009; Lai, 2006; Townsend \& Wan, 2007; Yu, 2010; Zhang, 2005). The refinements made to the SCAS in this research programme offer a more precise quantification or measurement of sojourner adjustment that, through its theoretically-driven and systematic approach, will allow researchers and practitioners working in these diverse disciplines the ability to more accurately study the processes and outcomes involved with cross-cultural behavioural competency. 
The research presented in this thesis also highlights conceptual advances to the acculturation model. Comprised of the stress and coping (affect), culture learning (behaviour), and social identification (cognition) frameworks, the conventional model of acculturation (Figure 1, page 6 of Chapter 1) infers that cognitions inform both affect and behaviours related to culture contact, but that this is not a mutually-informed or reciprocal relationship. In other words, the extant model does not consider how affect and behaviours may inform cognition. Although the ABC model of culture contact is situated on a metatheoretical level that does not explicitly consider specific sets of acculturative variables, this thesis offers an updated conceptualisation of acculturation to reflect the reciprocal influences of these three frameworks (Figure 11). More specifically, it is proposed that reciprocal relationships exist between: (1) The stress and coping, social identification, and culture learning frameworks; (2) the processes involved with coping with culture change; developing, maintaining, and changing identity, and acquiring specific behavioural skills; and (3) the acculturative outcomes of these processes and related frameworks. Further research is necessary to clarify the causal pathways or relational models between these different components of acculturation.

\section{Limitations and Future Directions}

Various methodological and instrumentation issues placed some constraints on the current thesis. For instance, all of the research reported in this research programme involved measures of self-report, creating certain restrictions around how the results may be interpreted and generalised. As an example, the degree of behavioural competency an individual exhibits in a new cultural setting can be considered as both an objective and subjective concept. In an objective sense, behavioural competency can be demonstrated via performance indicators or through observational indices (see Ruben \& Kealey, 1979). Subjectively, cross-cultural behavioural competency involves the degree to which an individual judges him or herself able to demonstrate such competency. Although researchers often rely heavily on self-report responses as a way of measuring competency, reliance on subjective accounts of performance or competency are best countered by objective or independent measures of performance. Future research could add an objective measure of competency through third person accounts (e.g., supervisor or peer evaluations) or perhaps 
through experimental designs that manipulate or prime an individual's degree of behavioural competency in novel social or cultural situations.

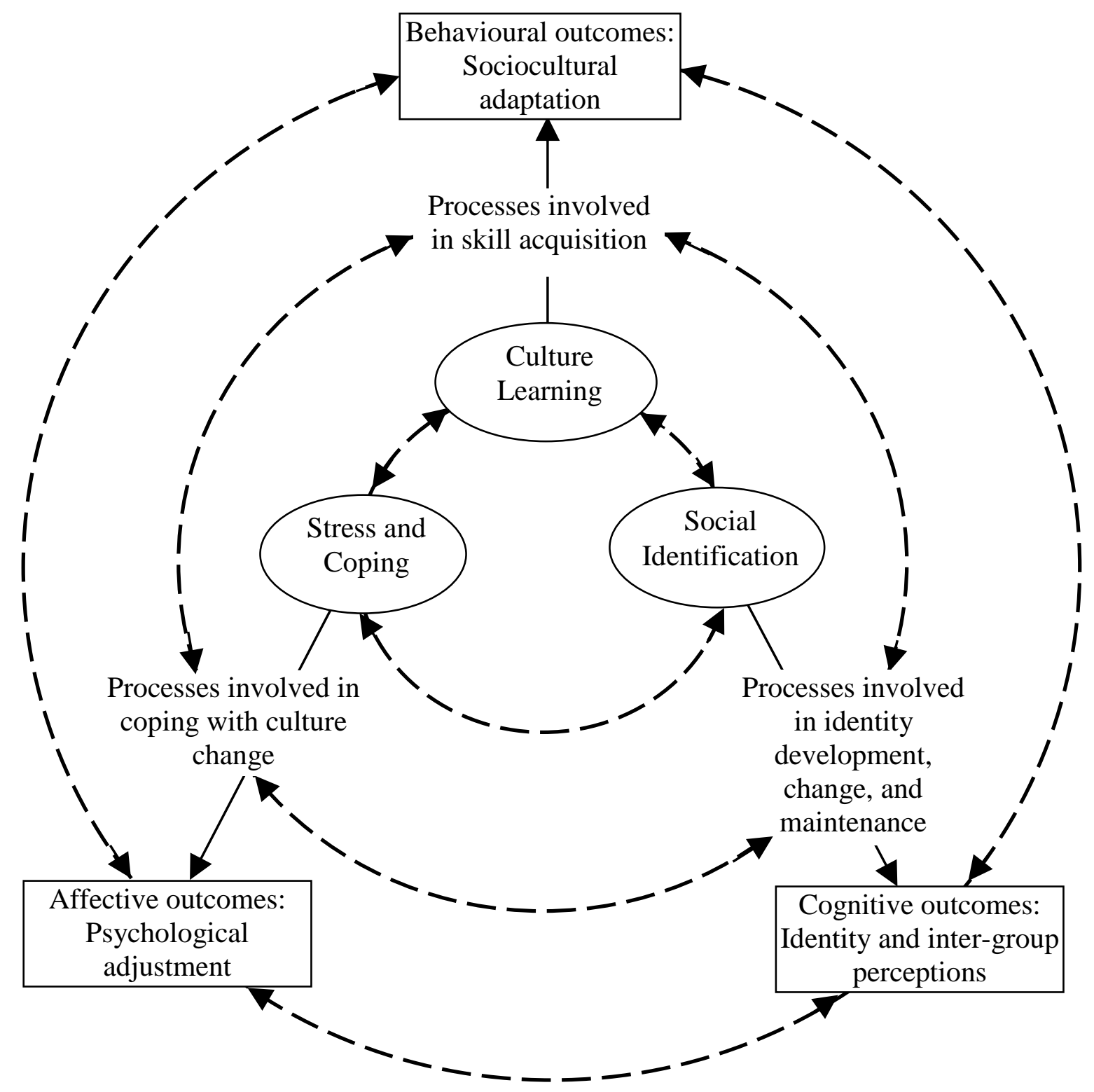

Figure 11. Adapted ABC model of culture contact.

Furthermore, development of the SCAS-R is still in its nascent stages, which involves continued exploration of various psychometric issues. As an example, the relationship between psychological adjustment indices such as life satisfaction and depression with sociocultural adaptation as measured by the SCAS-R requires further study. Previous research has shown that the magnitude of the relationship between psychological and sociocultural adaptation can change, particularly in conditions involving greater levels of an individual's cultural and social integration or when migrants or sojourning individuals are 
culturally similar rather than dissimilar to hosts (Ward, 2004). The magnitude of the psychological-sociocultural adjustment relationship is also stronger in sedentary groups as opposed to migrant groups, and stronger with individuals who have reported assimilationist or integrationist strategies of acculturation compared to those reporting separated and marginalised approaches (Ward et al., 1998; Ward \& Kennedy, 1996). Continued work with different migrant groups is necessary to ascertain the extent to which the SCAS-R relates to other indices of psychological well-being.

The precise nature of the SCAS-R factor structure also requires further scrutiny. It is evident from Studies 2 and 3 that various domains of adjustment exist that include participatory, non-verbal communication, linguistic, and ecological adaptation competencies involved with successful cross-cultural adaptation. Because both four- and five-factor structures of the SCAS-R were found in the current research programme, continued replication and validation work on the instrument is required using more heterogeneous participant samples. In addition to verifying the composition of these adaptation domains, supplementary research could also provide contextual information about what sociocultural areas of adjustment individuals find more challenging than others, delineate the discrepant antecedents and outcomes of these domains, or contribute to a more comprehensive understanding of how behavioural competency changes dependent upon differing domains individuals experience in a host society.

A final methodological limitation that must be addressed concerns causality. As was mentioned in the previous chapter, causality among variables based on cross-sectional data cannot be assumed. Ultimately, longitudinal or experimental designs are the only methodological approaches in which causality may be inferred. Future work concerning the topics covered in this research programme utilising these alternative methodologies would be particularly beneficial, as results would help obtain clearer, more empirically sound knowledge concerning the acculturation process.

Last, future research could examine the learning processes involved in behavioural skill acquisition. This thesis has highlighted the role learning plays in the process of behavioural skill acquisition, but further investigation of the stages of behavioural crosscultural competency and skill development is recommended. Various models exist that could be utilised for such endeavours, including frameworks around intercultural models of expertise and competence development (see Bhawuk, 1998; Bhawuk, Sakuda, \& Munusamy, 2008; Howell, 1986; Howell \& Fleishman, 1982). 


\section{Conclusion}

International migration trends have heralded a marked increase in intercultural contact, creating a greater need for effective cultural competency in both inter- and intracultural situations. Adroit, culturally competent individuals must balance a triangulation of factors in order to successfully navigate these changes within our societies and across our country borders. Cross-cultural competency requires becoming familiar with the unfamiliar, developing respect for different ideologies and behaviours that may contradict or challenge expectations and assumptions, and the confidence and motivation to persevere through challenges or issues that arise as a result of culture contact. Cross-cultural competency also entails the ability to function in and adapt to new surroundings. The research programme presented here has examined this latter aspect of intercultural competency. Through the review, revision, and expansion of the sociocultural adaptation construct, this research has aimed to provide a more thorough understanding of sojourner adaptation and settlement. Ultimately, the issues that arise from cross-cultural contact and cultural diversity have created an ever-growing demand for intercultural competence. The research outlined in this thesis offers those concerned with developing and improving intergroup relations new ways in which to better meet these demands. 


\section{References}

Abbe, A., Gulick, L.M.V., \& Herman, J.L. (2008). Cross-cultural competence in army leaders: A conceptual and empirical foundation. Intercultural Communication. Arlington, VA: U.S. Army Research Institute.

Abdul Malek, M., \& Budhwar, P. (2012). Cultural intelligence as a predictor of expatriate adjustment and performance in Malaysia. Journal of World Business. doi:10.1016/j.jwb.2012.07.006

Abe, H., \& Wiseman, R.L. (1983). A cross-cultural confirmation of the dimensions of intercultural effectiveness. International Journal of Intercultural Relations, 7, 53-67.

Adams, R.B., Rule, N.O., Franklin, R.G., Wang, E., Stevenson, M.T., Yoshikawa, S., Nomura, M., Sato, W., Kveraga, K., Ambady, N. (2009). Cross-cultural reading the mind in the eyes: An fMRI investigation. Journal of Cognitive Neuroscience, 22(1), 97108.

Adelman, M. (1988). Cross-cultural adjustment: A theoretical perspective on social support. International Journal of Intercultural Relations, 12, 183-204.

Adler, N.J. (1986). Do MBAs want international careers? International Journal of Intercultural Relations, 10(3), 277-300. doi:10.1016/0147-1767(86)90014-3

Ali, A., Van der Zee, K., \& Sanders, G. (2003). Determinants of intercultural adjustment among expatriate spouses. International Journal of Intercultural Relations, 27(5), 563580. doi:10.1016/S0147-1767(03)00054-3

Allison, K.W. (1998). Stress and oppressed social category membership. In J.K. Swim \& C. Stangor (Eds.), Prejudice: The target's perspective (pp. 145-170). San Diego, CA: Academic Press.

Allport, G.W. (1954). The nature of prejudice. Reading: Addison-Wesley.

Amit, K., \& Litwin, H. (2009). The subjective well-being of immigrants aged 50 and older in Israel. Social Indicators Research, 98(1), 89-104. doi:10.1007/s11205-009-9519-5

Anderson, J.C., \& Gerbing, D.W. (1984). The effect of sampling error on convergence, improper solutions, and goodness-of-fit indices for maximum likelihood confirmatory factor analyses. Psychometrika, 49(2), 155-173.

Anderson, P.A., Lustig, M.W., \& Anderson, J.F. (1990). Changes in latitude, changes in attitude: The relationship between climate and interpersonal communication predispositions. Communication Quarterly, 38, 291-311. 
Anderzen, I.I., \& Arnetz, B.B. (1999). Psychophysiological reactions to international adjustment: Results from a controlled, longitudinal study. Psychotherapy and Psychosomatics, 68, 67-75.

Ang, S., Van Dyne, L., \& Koh, C. (2006). Personality correlates of the four-factor model of cultural intelligence. Group and Organization Management, 31(1), 100-123. doi:10.1177/1059601105275267

Ang, S., Van Dyne, L., Koh, C., \& Ng, K.Y. (2004). The measurement of cultural intelligence. Academy of Management Meetings Symposium on Cultural Intelligence in the 21st Century. New Orleans, LA.

Ang, S., Van Dyne, L., Koh, C., Ng, K.Y., Templer, K.J., Tay, C., \& Chandrasekar, N.A. (2007). Cultural intelligence: Its measurement and effects on cultural judgment and decision making, cultural adaptation and task performance. Management and Organization Review, 3(3), 335-371. doi:10.1111/j.1740-8784.2007.00082.x

Angel, J.L., \& Angel, R.J. (1992). Age at migration, social connections, and well-being among elderly hispanics. Journal of Aging and Health, 4(4), 480-499. doi:10.1177/089826439200400402

Arbuckle, J. (2009). Amos 18 user's guide. SPSS Incorporated.

Archer, D. (1997). Unspoken diversity: Cultural differences in gestures. Qualitative Sociology, 20(1), 79- 105.

Argyle, M. (1969). Social interaction. London: Meuthen and Co. Ltd.

Argyle, M. (1979). New developments in the analysis of social skills. In A. Wolfgang (Ed.). London: Academic Press.

Argyle, M., \& Cook, M. (1976). Gaze and mutual gaze. Cambridge: Cambridge University Press.

Argyle, M., Furnham, A., \& Graham, J.A. (1981). Social situations. Cambridge: Cambridge University Press.

Argyle, M., \& Kendon, A. (1967). The experimental analysis of the social performance. In L. Berkowitz (Ed.), Advances in experimental social psychology (3rd ed., Vol. 3, pp. 5598). New York, NY: Academic Press.

Armes, K., \& Ward, C. (1989). Cross-cultural transitions and sojourner adjustment in Singapore. The Journal of Social Psychology, 129(2), 273-5. doi:10.1080/00224545.1989.9711728

Aronowitz, M. (1984). The social and emotional adjustment of immigrant children: A review of the literature. International Migration Review, 26, 86-110. 
Arthur, N., \& Achenbach, K. (2002). Developing multicultural counseling competencies through experiential learning. Counselor Education and Supervision, 42, 2-14.

Arthur, W., \& Bennett, W. (1995). The international assignee: The relative importance of factors perceived to contribute to success. Personnel Psychology, 48, 99-114.

Ashton, M.C. (1998). Personality and job performance: the importance of narrow traits. Journal of Organizational Behavior, 19(3), 289-303. doi:10.1002/(SICI)10991379(199805)19:3<289::AID-JOB841>3.0.CO;2-C

Ataca, B., \& Berry, J.W. (2002). Psychological, sociocultural, and marital adaptation of Turkish immigrant couples in Canada. International Journal of Psychology, 37, 13-26.

Augustine, V., Longmore, M., Ebenezer, M., \& Richard, J. (2012). Effectiveness of social skills training for reduction of self-perceived stigma in leprosy patients in rural India: A preliminary study. Leprosy Review, 83(1), 80-92.

Aycan, Z. (2008). Expatriate adjustment as a multifaceted phenomenon: Individual and organizational level predictors. The International Journal of Human Resource Management, 8(4), 434-456. doi:10.1080/095851997341540

Baard, P., Deci, E.L., \& Ryan, R.M. (2004). Intrinsic need satisfaction: A motivational basis of performance and well-being in two work settings. Journal of Applied Social Psychology, 34(10), 2045-2068. doi:10.1111/j.1559-1816.2004.tb02690.x

Babiker, I.E., Cox, J.L., \& Miller, P.M. (1980). The measurement of cultural distance and its relationship to medical consultations, symptomatology and examination performance of overseas students at Edinburgh University. Social Psychology, 15, 109-116.

Bakker, W., Van Oudenhoven, J.P., \& Van der Zee, K.I. (2004). Attachment styles, personality, and Dutch emigrants' intercultural adjustment. European Journal of Personality, 18, 387-404.

Bandura, A. (1977). Social learning theory. Oxford: Prentice-Hall.

Bandura, A. (1986). Social foundations of thought and action: A social cognitive theory. Englewood Cliffs, NJ: Prentice-Hall.

Bandura, A. (1995). Exercise of personal and collective efficacy in changing societies. In A. Bandura (Ed.), Self-efficacy in changing societies. New York, NY: Cambridge University Press.

Bandura, A. (1997). Self-efficacy: The exercise of control. New York, NY: Freeman.

Bandura, A. (2002). Social cognitive theory in cultural context. Applied Psychology, 51(2), 269-290. doi:10.1111/1464-0597.00092 
Bandura, A. (2006). Toward a psychology of human agency. Perspectives on Psychological Science, 1(2), 164-180. doi:10.1111/j.1745-6916.2006.00011.x

Bauer, M., Priebe, S., Kürten, I., Gräf, K.-J., \& Baumgartner, A. (1994). Psychological and endocrine abnormalities in refugees from East Germany: Prolonged stress, psychopathology, and hypothalamic-pituitary-thyroid axis activity. Psychiatry Research, 51(1), 61-73. doi:10.1016/0165-1781(94)90047-7

Beaupré, M.G., \& Hess, U. (2006). An ingroup advantage for confidence in emotion recognition judgments: The moderating effect of familiarity with the expressions of outgroup members. Personality and Social Psychology Bulletin, 31(1), 16-26.

Benson, M. (2011). The movement beyond (lifestyle) migration: Mobile practices and the constitution of a better way of life. Mobilities, 6(2), 221-235. doi:10.1080/17450101.2011.552901

Benson, M., \& O'Reilly, K. (2009). Migration and the search for a better way of life: A critical exploration of lifestyle migration. The Sociological Review, 57(4), 608-625. doi:10.1111/j.1467-954X.2009.01864.X

Benson, P.G. (1978). Measuring cross-cultural adjustment: The problem of criteria. International Journal of Intercultural Relations, 2(1), 21-37.

Bentler, P.M. (1990). Comparative fit indexes in structural models. Psychological Bulletin, 107(2), 238-246. doi:10.1037/0033-2909.107.2.238

Bentler, P.M., \& Chou, C.-P. (1987). Practical issues in structural modeling. Sociological Methods and Research, 16(1), 78-117. doi:10.1177/0049124187016001004

Berger, C.R. (1979). Beyond initial interactions. In H. Gales \& R. St. Clair (Eds.), Language and social psychology. Oxford: Basil Blackwell.

Bernardin, H.J., \& Bownas, D.A. (Eds.). (1985). Personality assessment in organizations (pp. v-vii). New York, NY: Praeger.

Berno, T., \& Ward, C. (1998). Psychological and sociocultural adjustment of international students in New Zealand. Annual Conference of the Society for Australasian Social Psychology. Christchurch, New Zealand.

Berno, T., \& Ward, C. (2003). The cross-cultural and educational adaptation of Asian students in New Zealand. Report for Asia 2000.

Berry, J.W. (1970). Marginality, stress and ethnic identification in an acculturated Aboriginal community. Journal of Cross-Cultural Psychology, 1, 239-252.

Berry, J.W. (1976). Human ecology and cognitive style: Comparative studies in cultural and psychological adaptation. New York, NY: Sage. 
Berry, J.W. (1990). Psychology of acculturation. In J.W. Berry \& J.J. Berman (Eds.), Nebraska symposium on motivation, 1989: Cross-cultural perspectives. Current theory and research in motivation (pp. 201-234). Lincoln, NE: University of Nebraska Press.

Berry, J.W. (1995). Psychology of acculturation. In N.R. Goldberger \& J. Veroff (Eds.), The culture and psychology reader (pp. 457-488). Newbury Park, CA: Sage.

Berry, J.W. (1997). Immigration, acculturation, and adaptation. Applied Psychology, 46(1), 5-34. doi:10.1111/j.1464-0597.1997.tb01087.x

Berry, J.W. (2001). Contextual studies of cognitive adaptation. In J.M. Collis \& S. Messick (Eds.), Intelligence and personality: Bridging the gap in theory and measurement (pp. 319-333). Mahwah, NJ: Lawrence Erlbaum.

Berry, J.W. (2006). Stress perspectives on acculturation. In D.L. Sam \& J.W. Berry (Eds.), Handbook of Acculturation Psychology (pp. 43-56). Cambridge, NY: Cambridge University Press.

Berry, J.W. (2009). A critique of critical acculturation. International Journal of Intercultural Relations, 33, 361-371.

Berry, J.W., \& Kim, U. (1988). Acculturation and mental health. In P. Dasen, J.W. Berry, \& N.Satorious (Eds.), Health and cross-cultural psychology: Towards applications. London: Sage.

Berry, J.W., Kim, U., Minde, T., \& Mok, D. (1987). Comparative studies of acculturative stress. International migration review, 21(3), 491-511.

Berry, J.W., Phinney, J.S., Sam, D.L., \& Vedder, P. (2006). Immigrant youth: Acculturation, identity, and adaptation. Applied psychology: An international review, 55(3), 303-332.

Berry, J.W., Poortinga, Y., Breugelmans, S., Chasiotis, A., \& Sam, D. (2011). Cross-cultural psychology: Research and applications (3rd ed.). Cambridge: Cambridge University Press.

Berry, J.W., Poortinga, Y.H., Segall, M.H., \& Dasen, P.R. (1992). Cross-cultural psychology: Research and application. New York, NY: Cambridge University Press.

Berscheid, E. (1985). Interpersonal attraction. Handbook of social psychology (3rd ed., pp. 413-484). New York, NY: Random House.

Bhawuk, D.P.S. (1998). The role of culture theory in cross-cultural training: A multimethod study of culture-specific, culture-general, and culture theory-based assimilators. Journal of Cross-Cultural Psychology, 29(5), 630-655. doi:10.1177/0022022198295003

Bhawuk, D.P.S., Sakuda, K.H., \& Munusamy, V.P. (2008). Intercultural competence development and triple-loop cultural learning: Toward a theory of intercultural 
sensitivity. In S. Ang \& L. van Dyne (Eds.), (pp. 342-355). Armonk, NY: M.E. Sharpe, Inc.

Bhawuk, D.P.S., \& Triandis, H.C. (1996). The role of culture theory in the study of culture and intercultural training. In D. Landis \& R. Bhagat (Eds.), Handbook of intercultural training (pp. 17-34). Newbury Park, CA: Sage.

Black, J.S. (1988). Work role transitions: A study of American expatriate managers in Japan, 19(2), 277-294.

Black, J.S., \& Gregersen, H.B. (1990). Expectations, satisfaction, and intention to leave of American expatriate managers in Japan. International Journal of Intercultural Relations, 14(4), 485-506.

Black, J.S., Gregersen, H.B., \& Mendenhall, M. (1992a). Toward a theoretical framework of repatriation adjustment. Journal of International Business Studies, 23(4), 737-760.

Black, J.S., \& Mendenhall, M. (1990). Cross-cultural training effectiveness: A review and a theoretical framework for future research. The Academy of Management Review, 15(1), $113-136$.

Black, J.S., Mendenhall, M., \& Oddou, G. (1991). Toward a comprehensive model of international adjustment: An integration of multiple theoretical perspectives. Academy of Management Review, 15(2), 291-317.

Black, J.S., \& Stephens, G.K. (1989). The influence of the spouse on American expatriate adjustment. Journal of Management, 15(4), 529-544. doi:10.1177/014920638901500403

Blake, B.F., Heslin, R., \& Curtis, S.C. (1996). Measuring impacts of cross-cultural training. In D. Landis \& R.S. Bhagat (Eds.), Handbook of Intercultural Training (2nd ed., pp. 165-182). Thousand Oaks, CA: Sage Publications, Inc.

Blume, B.D., Ford, J.K., Baldwin, T.T., \& Huang, J.L. (2009). Transfer of training: A metaanalytic review. Journal of Management, 36(4), 1065-1105. doi:10.1177/0149206309352880

Bochner, S. (1972). Problems in culture learning. In S. Bochner \& P. Wicks (Eds.), Overseas students in Australia (pp. 65-81). Sydney: University of New South Wales Press.

Bochner, S. (Ed.). (1981). The mediating person: Bridges between cultures. Boston, MA: G.K. Hall and Co.

Bochner, S., McLeod, B.M., \& Lin, A. (1977). Friendship patterns of overseas students: A function model. International Journal of Psychology, 12(4), 277-297. 
Bollen, K.A. (1989). A new incremental fit index for general structural equation models. Sociological Methods and Research, 17(3), 303-316. doi:10.1177/0049124189017003004

Bollen, K.A., \& Stine, R. (1990). Direct and indirect effects: Classical and bootstrap estimates of variability. Sociological Methodology, 20(1), 15-140.

Boneva, B.S., \& Frieze, I.H. (2001). Toward a concept of a migrant personality. Journal of Social Issues, 57(3), 477-491. doi:10.1111/0022-4537.00224

Boomsma, A. (2000). Reporting analyses of covariance structures. Structural Equation Modeling: A $\quad$ Multidisciplinary Journal, 7(3), 461-483. doi:10.1207/S15328007SEM0703_6

Booth, A., Crouter, A.C., \& Landale, N. (1997). Immigration and the family: Research and policy on U.S. immigrants. Mahwah, NJ: Lawrence Erlbaum Associates, Inc.

Bourhis, R.Y., Moïse, L.C., Perreault, S., \& Senecal, S. (1997). Towards an interactive acculturation model: A social psychological approach. International Journal of Psychology, 32, 369-386.

Brein, M., \& David, K.H. (1971). Intercultural communication and the adjustment of the sojourner. Psychological Bulletin, 76(3), 215-230. doi:10.1037/h0031441

Brenner, B.R. (2003). A study of self-awareness, self-efficacy, and sojourner adjustment over time. Unpublished doctoral dissertation. University of Maryland, College Park.

Brislin, R.W. (1981). Cross-cultural encounter: Face-to-face interaction. New York, NY: Pergamon.

Brislin, R.W., \& Yoshida, T. (Eds.). (1994). Intercultural communication training: An introduction. Thousand Oaks, CA: Sage Publications.

Bronfenbrenner, U. (1977). Toward an experimental ecology of human development. American Psychologist, 32(7), 513-531.

Bronfenbrenner, U. (1979). The ecology of human development: Experiments by nature and design. Cambridge, MA: Harvard University Press.

Brown, R. (2000). Social Identity Theory: Past achievements, current problems and future challenges. European Journal of Social Psychology, 30, 745-778.

Brown, T.A. (2006). Confirmatory factor analysis for applied research. New York, NY: Guilford.

Bryant, B., \& Trower, P. (1974). Social difficulty in a student sample. British Journal of Educational Psychology, 44, 13-21.

Buck, R.W. (1984). The communication of emotion. New York, NY: Guilford. 
Bull, R., \& Gibson-Robinson, E. (1981). The influences of eye-gaze, style of dress, and locality on the amounts of money donated to a charity. Human Relations, 34(10), 895905. doi:10.1177/001872678103401005

Burgoon, J.K. (1978). A communication model of personal space violations. Human Communication Research, 4, 129-142.

Burgoon, J.K. (1995). Cross-cultural and intercultural applications of expectancy violations theory. In R.L. Wiseman (Ed.), Intercultural communication theory (pp. 194-214). Thousand Oaks, CA: Sage.

Burton, K.D., Lydon, J.E., D’Alessandro, D.U., \& Koestner, R. (2006). The differential effects of intrinsic and identified motivation on well-being and performance: Prospective, experimental, and implicit approaches to self-determination theory. Journal of Personality and Social Psychology, 91(4), 750-62. doi:10.1037/0022-3514.91.4.750

Byrne, B.M. (1989). A primer of LISREL: Basic applications and programming for confirmatory factor analytic models. New York, NY: Springer-Verlag.

Byrne, B.M. (2001). Structural equation modeling with AMOS, EQS, and LISREL: Comparative approaches to testing for the factorial validity of a measuring instrument. International Journal of Testing, 1(1), 55-86. doi:10.1207/S15327574IJT0101_4

Byrne, B.M. (2010). Structural equation modeling with AMOS: Basic concepts, applications, and programming. New York, NY: Routledge.

Caligiuri, P.M. (2000). The Big Five personality characteristics as predictors of expatriate's desire to terminate the assignment and supervisor-rated performance. Personnel Psychology, 53(1), 67-88.

Caligiuri, P.M., Hyland, M.M., Joshi, A., \& Bross, A.S. (1998). Testing a theoretical model for examining the relationship between family adjustment and expatriates' work adjustment. Journal of Applied Psychology, 83(4), 598-614. doi:10.1037/00219010.83.4.598

Caligiuri, P.M., \& Tung, R.L. (1999). Comparing the success of male and female expatriates from a US-based multinational company. The International Journal of Human Resource Management, 10(5), 763-782. doi:10.1080/095851999340143

Campbell, D.T., \& Fiske, D.W. (1959). Convergent and discriminant validation by the multitrait-multimethod matrix. Psychological Bulletin, 56(2), 81-105. doi: $10.1037 / \mathrm{h} 0046016$

Campbell, J.P. (1999). The definition and measurement of performance in the new age. In D.R. Ilgen \& E.D. Pulakos (Eds.), The changing nature of performance: Implications 
for staffing, motivation, and development (pp. 399-429). San Francisco, CA: JosseyBass.

Caprara, G.V., Vecchione, M., Alessandri, G., Gerbino, M., \& Barbaranelli, C. (2011). The contribution of personality traits and self-efficacy beliefs to academic achievement: A longitudinal study. The British Journal of Educational Psychology, 81(1), 78-96. doi:10.1348/2044-8279.002004

Cassidy, C., \& O’Connor, R.C. (2005). Perceived discrimination among ethnic minority young people: The role of psychological variables. Journal of Applied Social Psychology, 35, 1246-1265.

Castillo, L.G., Conoley, C.W., Brossart, D.F., \& Quiros, A.E. (2007). Construction and validation of the Intragroup Marginalization Inventory. Cultural Diversity and Ethnic Minority Psychology, 13(3), 232-40. doi:10.1037/1099-9809.13.3.232

Castro, V.W. (2003). Acculturation and psychological adaptation. Westport, CT: Greenwood Press.

Chao, M.M., Okazaki, S., \& Hong, Y.-Y. (2011). The quest for multicultural competence: Challenges and lessons learned from clinical and organizational research. Social and Personality Compass, 5, 263-274.

Chen, W. (2010). Internet-usage patterns of immigrants in the process of intercultural adaptation. Cyberpsychology, Behavior and Social Networking, 13(4), 387-99. doi:10.1089/cyber.2009.0249

Chen, X.-P., Liu, D., \& Portnoy, R. (2012). A multilevel investigation of motivational cultural intelligence, organizational diversity climate, and cultural sales: Evidence from U.S. real estate firms. The Journal of Applied Psychology, 97(1), 93-106. doi:10.1037/a0024697

Cheung, F.M. (1996). The assessment of psychopathology in Chinese societies. In M.H. Bond (Ed.), The Handbook of Chinese Psychology (pp. 393-411). Hong Kong: Oxford University Press.

Cheung, F.M., \& Leung, K. (1998). Indigenous personality measures: Chinese examples. Journal of Cross-Cultural Psychology, 29(1), 233-248. doi:10.1177/0022022198291012

Chirkov, V.I. (2009a). Introduction to the special issue on critical acculturation psychology. International Journal of Intercultural Relations, 33, 87-93.

Chirkov, V.I. (2009b). Critical psychology of acculturation: What do we study and how do we study it, when we investigate acculturation? International Journal of Intercultural Relations, 33(2), 94-105. 
Chirkov, V.I., Lynch, M., \& Niwa, S. (2005). Application of the scenario questionnaire of horizontal and vertical individualism and collectivism to the assessment of cultural distance and cultural fit. International Journal of Intercultural Relations, 29(4), 469490. doi:10.1016/j.ijintrel.2005.05.014

Chirkov, V.I., Safdar, S., De Guzman, J., \& Playford, K., (2008). Further examining the role motivation to study abroad plays in the adaptation of international students in Canada. International Journal of Intercultural Relations, 32(5), 427-440. doi:10.1016/j.ijintrel.2007.12.001

Chirkov, V.I., Vansteenkiste, M., Tao, R., \& Lynch, M. (2007). The role of self-determined motivation and goals for study abroad in the adaptation of international students. International Journal of Intercultural Relations, 31, 199-222.

Church, A.T. (1982). Sojourner adjustment. Psychological Bulletin, 76, 130-215.

Clark, L.A., \& Watson, D. (1995). Constructing validity: Basic issues in objective scale development. Psychological Assessment, 7(3), 309-319. doi:10.1037/1040-3590.7.3.309

Clark, R., Anderson, N.B., Clark, V.R., \& Williams, D.R. (1999). Racism as a stressor for African Americans: A biopsychosocial model. American Psychologist, 54(10), 805-816. doi:10.1037/0003-066X.54.10.805

Clément, R., Baker, S., \& MacIntyre, P.D. (2003). Willingness to communicate in a second language: The effects of context, norms, and vitality. Journal of Language and Social Psychology, 22(2), 190-209. doi:10.1177/0261927X03022002003

Clément, R., \& Bourhis, R.Y. (1996). Bilingualism and intergroup communication. International Journal of Psycholinguistics, 12, 171-191.

Clément, R., Gardner, R. C., \& Smythe, P. C. (1980). Social and individual factors in second language acquisition. Canadian Journal of Behavioural Science/Revue canadienne des sciences du comportement, 12(4), 293-302. doi:10.1037/h0081081

Cohen, J. (1988). Statistical power analysis for the behavioral sciences (2nd ed.). Hillsdale: Erlbaum.

Cohen, J., \& Cohen, P. (1983). Applied multiple regression/correlation analysis for the behavioral sciences (2nd ed.). Hillsdale, NY: Erlbaum.

Collett, P. (1971). On training Englishmen in the non-verbal behaviour of Arabs: An experiment in intercultural communication. International Journal of Psychology, 6, 209-215.

Collett, P. (1993). Foreign bodies: A guide to European mannerisms. London: Simon \& Schuster. 
Collier, M. J. (1989). Cultural and intercultural communication competence: Current approaches and directions for future research. International Journal of Intercultural Relations, 13(3), 287-302. doi:10.1016/0147-1767(89)90014-X

Comrey, A. L., \& Lee, H. B. (1992). A first course in factor analysis (2nd ed.). Hillsdale, NJ: Erlbaum.

Contrada, R. J., Ashmore, R. D., Gary, M. L., Coups, E., Egeth, J. D., Sewell, A., Ewell, K., et al. (2000). Ethnicity-related sources of stress and their effects on well-being. Current Directions in Psychological Science, 9(4), 136-139. doi:10.1111/1467-8721.00078

Cooke, T. J. (2001). "Trailing wife" or "trailing mother"? The effect of parental status on the relationship between family migration and the labor-market participation of married women. Environment and Planning A, 33(3), 419-430. doi:10.1068/a33140

Cort, D. A., \& King, M. (1979). Some correlates of culture shock among American tourists in Africa. International Journal of Intercultural Relations, 3(2), 211-225. doi:10.1016/0147-1767(79)90065-8

Costa, P. T., \& McCrae, R. R. (1992). NEO PI-R professional manual. Odessa, FL: Psychological Assessment Resources, Inc.

Costello, A.B., \& Osborne, J.W. (2005). Best practices in exploratory factor analysis: Four recommendations for getting the most from your analysis. Practical Assessment, Research and Evaluation, 10(7), 1-9.

Cowling, W.E. (2002). Motivations for contemporary Tongan migration. In P. R. Spickard, J. L. Rondilla, \& D. H. Wright (Eds.), Pacific diaspora: Island peoples in the United States and across the pacific (pp. 99-117). Honolulu, HI: University of Hawai'i Press.

Cox, J. (2004). The role of communication, technology, and cultural identity in repatriation adjustment. International Journal of Intercultural Relations, 28(3-4), 201-219. doi:10.1016/j.ijintrel.2004.06.005

Cronbach, L.J. (1951). Coefficient alpha and the internal structure of tests. Psychometrika, 16(3), 297-334. doi:10.1007/BF02310555

Culhane, S.F. (2001). Intercultural contact in a study-abroad ESL setting. University of British Columbia.

Culhane, S.F., \& Kimber, L. (2001). Contact motivation in a SLA setting: Applying the intercultural contact model to sojourners at a Japanese university. Polyglossia, 4, 31-39.

Dalhaug, K.C., Oppedal, B., \& Røysamb, E. (2011). The role of sociocultural context for culture competence and depressive symptoms among ethnic minority youths in junior 
high school. European Journal of Developmental Psychology, 8(3), 280-294. doi:10.1080/17405621003710843

Daly, A.J. (2007). Outbound student exchange at Australian and New Zealand universities: The effects of pre-departure decision-making, in-country experiences and post-sojourn outcomes. Unpublished doctoral dissertation. Griffith University.

Dasen, P.R., Berry, J.W., \& Sartorius, N. (1988). Health and cross-cultural psychology: Towards applications. Newbury Park, CA: Sage.

Davis, T.R.V., \& Luthans, F. (1980). A social learning approach to organizational behavior. The Academy of Management Review, 5(2), 281-290.

Deci, E.L., \& Ryan, R.M. (1985). Intrinsic motivation and self-determination in human behavior. New York, NY: Plenum.

Deci, E.L., \& Ryan, R.M. (2000). The "what" and "why" of goal pursuits: Human needs and the self-determination of behavior. Psychological Inquiry, 11(4), 227-268. doi:10.1207/S15327965PLI1104_01

Deller, J. (1997). Expatriate selection: Possibilities and limitations of using personality scales. In Z. Aycan \& D.M. Saunders (Eds.), New approaches to employee management, Vol. 4: Expatriate management: Theory and research (pp. 93-116). Greenwich, CT: JAI Press.

Deshpande, S.P., \& Viswesvaran, C. (1992). Is cross-cultural training of expatriate managers effective: A meta analysis. International Journal of Intercultural Relations, 16(3), 295310.

Diener, E., Emmons, R.A., Larsen, R.J., \& Griffin, S. (1985). The Satisfaction with Life Scale. Journal of Personality Assessment, 49(1), 71-5. doi:10.1207/s15327752jpa4901_13

Dion, K.L., \& Earn, B.M. (1975). The phenomenology of being a target of prejudice. Journal of Personality and Social Psychology, 32(5), 944-950. doi:10.1037/0022-3514.32.5.944

DiTommaso, E., Brannen-McNulty, C., Ross, L., \& Burgess, M. (2003). Attachment styles, social skills and loneliness in young adults. Personality and Individual Differences, 35(2), 303-312. doi:10.1016/S0191-8869(02)00190-3

Donato, K.M., Gabaccia, D., Holdaway, J., Manalansan, M., \& Pessar, P.R. (2006). A glass half full? Gender in migration studies. International Migration Review, 40(1), 3-26. doi:10.1111/j.1747-7379.2006.00001.x

Dorigo, G., \& Tobler, W. (1983). Push-pull migration laws. Annals of the Association of American Geographers, 73(1), 1-17. doi:10.1111/j.1467-8306.1983.tb01392.x 
Duckitt, J.H. (1992). Psychology and prejudice: A historical analysis and integrative framework. American Psychologist, 47(10), 1182-1193. doi:10.1037/0003066X.47.10.1182

Duru, E., \& Poyrazli, S. (2007). Personality dimensions, psychosocial-demographic variables, and English language competency in predicting level of acculturative stress among Turkish international students. International Journal of Stress Management, 14(1), 99-110. doi:10.1037/1072-5245.14.1.99

Earley, P.C., \& Ang, S. (2003). Cultural intelligence: Individual interactions across cultures. Stanford, CA: Stanford Business Books.

Earley, P.C. (2002). Redefining interactions across cultures and organizations: Moving forward with cultural intelligence. Research in Organizational Behavior, 24, 271-299. doi:10.1016/S0191-3085(02)24008-3

Education First (2011). English proficiency index 2011 report. Retrieved from http://www.ef.co.nz/sitecore/\%5C_\%5C_/ /media/efcom/epi/pdf/EF-EPI-2011.pdf

Efron, D. (1941). Gesture and environment. New York, NY: King's Crown Press.

Ekman, P. (1972). Universals and cultural differences in facial expressions of emotion. In J. Cole (Ed.), Nebraska Symposium on Motivation, 1971 (pp. 207-282). Lincoln, NE: University of Nebraska Press.

Ekman, P. (1992). An argument for basic emotions. Cognition \& Emotion, 6(3), 169-200. doi:10.1080/02699939208411068

Elenkov, D., \& Pimentel, J.R. (2008). Social intelligence, emotional intelligence, and cultural intelligence: An integrative perspective. In S. Ang \& L. Van Dyne (Eds.), Handbook of cultural intelligence: Theory, measurement, and applications (pp. 289-395). New York, NY: M.E. Sharpe, Inc.

Elfenbein, H.A., \& Ambady, N. (2003). When familiarity breeds accuracy: Cultural exposure and facial emotion recognition. Journal of Personality and Social Psychology, 85(2), 276-290. doi:10.1037/0022-3514.85.2.276

Enniger, W. (1987). What interactants do about non-talk across cultures. In K. Knapp, W. Ennger, \& A. Knapp-Potthoff (Eds.), Analyzing intercultural interactions. Berlin: Mouton de Gruyer.

Eshel, Y., \& Rosenthal-Sokolov, M. (2000). Acculturation attitudes and sociocultural adjustment of sojourner youth in Israel. The Journal of Social Psychology, 140(6), 677691. 
Ewers, M.C. (2007). Migrants, markets and multinationals: Competition among world cities for the highly skilled. GeoJournal, 68(2-3), 119-130. doi:10.1007/s10708-007-9077-9

Eysenck, S.B.G., Eysenck, H.J., \& Barrett, P. (1985). A revised version of the psychoticism scale. Personality and Individual Differences, 6(1), 21-29. doi:10.1016/01918869(85)90026-1

Fabrigar, L. R., Wegener, D. T., MacCallum, R. C., \& Strahan, E. J. (1999). Evaluating the use of exploratory factor analysis in psychological research. Psychological Methods, 4(3), 272-299. doi:10.1037/1082-989X.4.3.272

Familiar, I., Borges, G., Orozco, R., \& Medina-Mora, M.-E. (2011). Mexican migration experiences to the US and risk for anxiety and depressive symptoms. Journal of Affective Disorders, 130(1-2), 83-91. doi:10.1016/j.jad.2010.09.025

Fan, C., \& Mak, A.S. (1998). Measuring social self-efficacy in a culturally diverse student population. Social Behavior and Personality, 26(2), 131-144. doi:10.2224/sbp.1998.26.2.131

Farley, J.U., Lehmann, D.R., \& Sawyer, A. (1995). Empirical marketing generalization using meta-analysis. Special Issue on Empricial Generalizations in Marketing, 14(3), G36G46.

Fazel, M., Wheeler, J., \& Danesh, J. (2005). Prevalence of serious mental disorder in 7000 refugees resettled in western countries: A systematic review. Lancet, 365(9467), 13091314.

Feldman Barrett, L., \& Swim, J.K. (1998). Appraisals of prejudice and discrimination. In J.K. Swim \& C. Strangor (Eds.), Prejudice: The target's perspective (pp. 11-36). San Diego, CA: Academic Press.

Feldman, D.C. (1976). A contingency theory of socialization. Administrative Science Quarterly, 21(3), 433-452.

Field, A.P. (2001). Meta-analysis of correlation coefficients: A Monte Carlo comparison of fixed- and random-effects methods, Psychological Methods, 6(2), 161-180.

Field, A.P. (2003). The problems in using fixed-effects models of meta-analysis on realworld data, Understanding Statistics: Statistical Issues in Psychology, Education, and the Social Sciences, 2(2), 105-124.

Field, A.P. (2005). Discovering statistics using SPSS (2nd ed.). London: Sage.

Fielder, F., Mitchell, T., \& Triandis, H. (1971). The culture assimilator: An approach to cross-cultural training. Journal of Applied Psychology, 55, 95-102. 
Fisher, R.A. (1915). Frequency distribution of the values of the correlation coefficient in samples of an indefinitely large population. Biometrika, 10(4), 507-521.

Fontaine, G. (1986). Roles of social support in overseas relocation: Implications for intercultural training. International Journal of Intercultural Relations, 10, 361-378.

Forced Migration Online (FMO). (2012). What is forced migration? Retrieved February 11, 2012, from http://www.forcedmigration.org/about/whatisfm

Fowler, S.M. (1994). Two decades of using simulation games for cross-cultural training. Simulation \& Gaming, 25(4), 464-476. doi:10.1177/1046878194254004

Fox, S.H. (2010). Ancient ways in current days: Ethno-cultural arts and acculturation. Unpublished doctoral dissertation. Victoria University of Wellington.

Frazier, P.A., Tix, A.P., \& Barron, K.E. (2004). Testing moderator and mediator effects in counseling psychology research. Journal of Counseling Psychology, 51(1), 115-134. doi:10.1037/0022-0167.51.1.115

Furnham, A., \& Bochner, S. (1982a). Cultures in contact: Studies in cross-cultural interaction. Oxford: Pergamon Press.

Furnham, A., \& Bochner, S. (1982b). Social difficulty in a foreign culture: An empirical analysis of culture shock. In S. Bochner (Ed.), Cultures in contact: Studies in crosscultural interaction (Vol. 1, pp. 161-198). Oxford: Pergamon Press.

Furnham, A., \& Bochner, S. (1986). Culture shock: Psychological reactions to unfamiliar environments. London: Methuen.

Furnham, A., \& Erdman, S. (1995). Psychological and socio-cultural variables as predictors of adjustment in cross-cultural transitions. Psychologia, 38, 238-251.

Furnham, A., \& Trezise, L. (1981). The mental health of foreign students. Social Science and Medicine, 17, 365-370.

Furukawa, T., \& Shibayama, T. (1993). Predicting maladjustment of exchange students in different cultures: A prospective study. Epidemiology, 28(3), 142-146. doi:10.1007/BF00801745

Galchenko, I., \& Van de Vijver, F.J. (2007). The role of perceived cultural distance in the acculturation of exchange students in Russia. International Journal of Intercultural Relations, 31, 181-197.

Gallois, C., Franklyn-Stokes, A., Giles, H., \& Coupland, N. (1988). Communication accommodation theory and intercultural encounters: Intergroup and interpersonal considerations. In Y.Y. Kim \& W.B. Gudykunst (Eds.), International and intercultural 
communication annual, Vol. XII: Theories in intercultural communication (pp. 157185). Newbury Park, CA: Sage Publications.

Gallois, C., Giles, H., Jones, E., Cargile, A., \& Ota, H. (1995). Accommodating intercultural encounters: Elaborations and extensions. In R.L. Wiseman (Ed.), Intercultural communication theory (pp. 115-147). Thousand Oaks, CA: Sage.

Gao, G., \& Gudykunst, W.B. (1990). Uncertainty, anxiety and adaptation. International Journal of Intercultural Relations, 14, 301-317.

García Coll, C, Crnic, K., Lamberty, G., Wasik, B.H., Jenkins, R., Garcia, H.V., \& McAdoo, H.P. (1996). An integrative model for the study of developmental competencies in minority children. Child Development, 67(5), 1891-1914. doi:10.1111/j.14678624.1996.tb01834.x

Gardner, R.C. (1985). Social psychology and second language learning: The role of attitudes and motivation. London: Edward Arnold.

Gardner, R.C. (2000). Correlation, causation, motivation, and second language acquisition. Canadian Psychology/Psychologie Canadienne, 41(1), 10-24. doi:10.1037/h0086854

Georgas, J. (1988). An ecological social model: The case of Greece. In J.W. Berry, S.H. Irvine, \& E.B. Hunt (Eds.), Indigenous cognition: Functioning in cultural context. Dordrecht: Nijhoff.

Georgas, J. (1993). Ecological-social model of Greek psychology. In U. Kim \& J.W. Berry (Eds.), Indigenous Psychologies (pp. 56-78). Thousands Oaks, CA: Sage Publications.

Georgas, J., Van de Vijver, F.J.R., \& Berry, J.W. (2004). The ecocultural framework, ecosocial indices, and psychological variables in cross-cultural research. Journal of Cross-Cultural Psychology, 35(1), 74-96. doi:10.1177/0022022103260459

Gertsen, M.C. (1990). Intercultural competence and expatriates. The International Journal of Human Resource Management, 1(3), 341-362. doi:10.1080/09585199000000054

Gezentsvey, M., \& Ward, C. (2008). Unveiling agency: A motivational perspective on acculturation and adaptation. In R.M. Sorrentino \& S. Yamaguchi (Eds.), Handbook of motivation and cognition across cultures. San Diego, CA: Elsevier Inc.

Giddens, A. (1991). Modernity and self-identity: Self and society in the late modern age. Stanford, CA: Stanford University Press.

Giles, H., Coupland, N., \& Wiemann, J.M. (1992). "Talk is cheap...” but "my word is my bond": Beliefs about talk. In K. Bolton \& H. Kwok (Eds.), Sociolinguists today: Eastern and Western perspectives (pp. 218-243). London: Routledge. 
Glass, G.V. (1976). Primary, secondary, and meta-analysis of research. Educational Researcher, 5(3), 308.

Goldberg, D. (1972). The detection of psychiatric illness by questionnaire. Oxford: Oxford University Press.

Gong, Y. (2003). Goal orientations and cross-cultural adjustment: An exploratory study. International Journal of Intercultural Relations, 27(3), 297-305. doi:10.1016/S01471767(03)00013-0

Gong, Y., \& Fan, J. (2006). Longitudinal examination of the role of goal orientation in crosscultural adjustment. The Journal of Applied Psychology, 91(1), 176-84. doi:10.1037/0021-9010.91.1.176

Gorsuch, R.L. (1983). Factor analysis (2nd ed.). Hillsdale, NJ: Erlbaum.

Goss, J., \& Linquist, B. (1995). Conceptualizing international labor migration: A structuration perspective. International Migration Review, 29(2), 317-351.

Gudykunst, W.B. (1999). Theory and research on intercultural relations: An introduction. International Journal of Intercultural Relations, 23(4), 529-534.

Gudykunst, W.B., \& Hammer, M.R. (1988). Strangers and hosts: An uncertainty reduction based theory of intercultural adaptation. In Y.Y. Kim \& W.B. Gudykunst (Eds.), Crosscultural adaptation: Current approaches (pp. 106-139). Newbury Park, CA: Sage.

Gudykunst, W.B., Hammer, M.R., \& Wiseman, R.L. (1977). An analysis of an integrated approach to cross-cultural training. International Journal of Intercultural Relations, 1(2), 99-110. doi:10.1016/0147-1767(77)90045-1

Gudykunst, W.B., \& Kim, Y.Y. (1984). Communicating with strangers: An approach to intercultural communication. New York, NY: Random House.

Gudykunst, W.B., \& Matsumoto, Y. (1996). Cross-cultural variability of communication in personal relationships. In W.B. Gudykunst, S. Ting-Toomey, \& T. Nashida (Eds.), Communication in personal relationships across cultures. Thousand Oaks, CA: Sage Publications, Inc.

Gullahorn, J.E., \& Gullahorn, J.T. (1966). American students abroad: Professional versus personal development. The Annals of the American Academy of Political and Social Science, 368(1), 43-59. doi:10.1177/000271626636800106

Gullahorn, J.T., \& Gullahorn, J.E. (1963). An extension of the u-curve hypothesis. Journal of Social Issues, 19(3), 33-47. doi:10.1111/j.1540-4560.1963.tb00447.x 
Gungor, D., \& Bornstein, M.H. (2009). Gender, development, values, adaptation, and discrimination in acculturating adolescents: The case of Turk heritage youth born and living in Belgium. Sex Roles, 60, 537-548.

Hall, E.T. (1976). Beyond culture. Garden City, NJ: Doubleday.

Hall, E.T., \& Hall, M.R. (1990). Understanding cultural differences: Germans, French and Americans. London: Nicholas Brealey Publishing.

Hammer, M.R. (1987). Behavioral dimensions of intercultural effectiveness: A replication and extension. International Journal of Intercultural Relations, 11(1), 65-88. doi:10.1016/0147-1767(87)90032-0

Hammer, M.R., Gudykunst, W., \& Wiseman, R.L. (1978). Dimensions of intercultural effectiveness: An exploratory study. International Journal of Intercultural Relations, 2, $382-393$.

Hannigan, T.P. (1990). Traits, attitudes, and skills that are related to intercultural effectiveness and their implications for cross-cultural training: A review of the literature. International Journal of Intercultural Relations, 14(1), 89-111.

Hardre, P.L., \& Reeve, J.M. (2003). A motivational model of rural students' intentions to persist in, versus drop out of, high school. Journal of Educational Psychology, 95(2), 347-356. doi:10.1037/0022-0663.95.2.347

Harrison, J.K., Chadwick, M., \& Scales, M. (1996). The relationship between cross-cultural adjustment and the personality variables of self-efficacy and self-monitoring. International Journal of Intercultural Relations, 20(2), 167-188.

Hasegawa, T., \& Gudykunst, W.B. (1998). Silence in Japan and the United States. Journal of Cross-Cultural Psychology, 29(5), 668-684. doi:10.1177/0022022198295005

Hassell, B.L., \& Perrewé, P.L. (1993). An examination of the relationship between older workers' perceptions of age discrimination and employee psychological states. Journal of Managerial Issues, 5(1), 109-120.

Hatcher, L. (1994). A step-by-step approach to using SAS for factor analysis and structural equation modeling. Cary, NC: SAS Institute, Inc.

Hawes, F., \& Kealey, D.J. (1981). An empirical study of Canadian technical assistance. International Journal of Intercultural Relations, 5(3), 239-258. doi:10.1016/01471767(81)90028-6

Hayduk, L.A. (1983). Personal space: Where we now stand. Psychological Bulletin, 94(2), 293-335. doi:10.1037/0033-2909.94.2.293 
Hechanova, R. (2003). Antecedents and consequences of employees' adjustment to overseas assignment: A meta- analytic review. Applied Psychology.

Hedges, L.V. (1983). A random effects model for effect sizes. Psychological Bulletin, 93(2), 388-395. doi:10.1037/0033-2909.93.2.388

Hedges, L.V., \& Olkin, I. (1985). Statistical methods for meta-analysis. Orland, FL: Academic Press.

Hedges, L.V., \& Vevea, J.L. (1998). Fixed- and random-effects models in meta-analysis. Psychological methods, 3(4), 486-504.

Hilgard, E.R., \& Bower, G.H. (1975). Theories of learning. Englewood Cliffs, NJ: PrenticeHall.

Hiller, H.H., \& McCaig, K.S. (2007). Reassessing the role of partnered women in migration decision-making and migration outcomes. Journal of Social and Personal Relationships, 24(3), 457-472. doi:10.1177/0265407507077233

Hodgins, H.S., Weibust, K.S., Weinstein, N., Shiffman, S., Miller, A., Coombs, G., \& Adair, K.C. (2010). The cost of self-protection: Threat response and performance as a function of autonomous and controlled motivations. Personality and Social Psychology Bulletin, 36(8), 1101-14. doi:10.1177/0146167210375618

Hofstede, G. (1980). Culture's consequences: International differences in work-related values. London: Sage.

Hofstede, G. (1983). Dimensions of national cultures in fifty countries and three regions. In J. Deregowski, S. Dziurawiec, \& R. Annis (Eds.), Expiscations in cross-cultural psychology. Lisse: Swets and Zeitlinger.

Hofstede, G. (2001). Culture's consequences (2nd ed.). Thousand Oaks, CA: Sage.

Honna, N. (2005). English as a multicultural language in Asia and intercultural literacy. Intercultural Communication Studies, 14(2), 73-89.

Horenczyk, G. (1996). Migrant identities in conflict: Acculturation attitudes and perceived acculturation ideologies. In G. Breakwell \& E. Lyons (Eds.), Changing European identities: Social psychological analyses of social change (pp. 241-250). Oxford: Butterworth-Heinemann.

Horn, J.L. (1965). A rationale and test for the number of factors in factor analysis. Psychometrika, 30(2), 179-185. doi:10.1007/BF02289447

Hornik, J. (1987). The effect of touch and gaze upon compliance and interest of interviewees. The Journal of Social Psychology, 127, 681-683. 
Houlfort, N., Koestner, R., Joussemet, M., Nantel-Vivier, A., \& Lekes, N. (2002). The impact of performance-contingent rewards on perceived autonomy and competence. Motivation and Emotion, 26, 279-295.

Howard, G.S., Maxwell, S.E., Wiener, R.L., Boynton, K.S., \& Rooney, W.M. (1980). Is a behavioral measure the best estimate of behavioral parameters? Perhaps not. Applied Psychological Measurement, 4(3), 293-311. doi:10.1177/014662168000400302

Howell, W. (1986). The empathic communicator. Prospect Heights, IL: Waveland Press.

Howell, W.C., \& Fleishman, E.A. (Eds.). (1982). Human performance and productivity. Volume 2: Information processing and decision making. Hillsdale, NJ: Erlbaum.

Hu, L., \& Bentler, P.M. (1999). Cutoff criteria for fit indexes in covariance structure analysis: Conventional criteria versus new alternatives. Structural Equation Modeling: A Multidisciplinary Journal, 6(1), 1-55. doi:10.1080/10705519909540118

Hui, C.H., \& Triandis, H.C. (1985). Measurement in cross-cultural psychology: A review and comparison of strategies. Journal of Cross-Cultural Psychology, 16(2), 131-152. doi:10.1177/0022002185016002001

Hunter, J.E. (1987). Multiple dependent variables in program evaluation. New Directions for Program Evaluation, 35, 43-56. doi:10.1002/ev.1458

Hunter, J.E., \& Schmidt, F.L. (1990). Methods of meta-analysis: Correcting error and bias in research findings (p. 592). Thousand Oaks, CA: Sage.

Hutcheson, G., \& Sofroniou, N. (1999). The multivariate social scientist: Introductory statistics using generalized linear models (p. 276). London: Sage Publications Ltd.

Ilic, M., Reinecke, J., Bohner, G., Röttgers, H.O., Beblo, T., Driessen, M., Frommberger, U., \& Corrigan, P.W. (2012). Protecting self-esteem from stigma: A test of different strategies for coping with the stigma of mental illness. International Journal of Social Psychiatry, 58(3), 246-257.

IMSED Research. (2008). Life in New Zealand: Settlement Experiences of Skilled Migrants Results from the 2008 Survey. Department of Labour. Retrieved from http://www.worksite.co.nz/publications/research/settlement/Settlement-Experiences-ofSkilled-Migrants.doc

Ishiyama, F.I., \& Westwood, M.J. (1992). Enhancing client-validating communication:

Helping discouraged clients in cross-cultural adjustment. Journal of Multicultural Counseling and Development, 20(2), 50-63. doi:10.1002/j.2161-1912.1992.tb00562.x 
Jackson, D.L., Gillaspy, J.A., \& Purc-Stephenson, R. (2009). Reporting practices in confirmatory factor analysis: An overview and some recommendations. Psychological methods, 14(1), 6-23. doi:10.1037/a0014694

Jasinskaja-Lahti, I. (2008). Long-term immigrant adaptation: Eight-year follow-up study among immigrants from Russia and Estonia living in Finland. International Journal of Psychology, 43(1), 6-18. doi:10.1080/00207590701804271

Jasinskaja-Lahti, I., \& Liebkind, K. (2007). A structural model of acculturation and wellbeing among immigrants from the former USSR in Finland. European Psychologist, 12(2), 80-92. doi:10.1027/1016-9040.12.2.80

Jasinskaja-Lahti, I., Liebkind, K., Horenczyk, G., \& Schmitz, P. (2003). The interactive nature of acculturation: Perceived discrimination, acculturation attitudes and stress among young ethnic repatriates in Finland, Israel and Germany. International Journal of Intercultural Relations, 27(1), 79-97. doi:10.1016/S0147-1767(02)00061-5

Jaworski, A. (1993). The power of silence. Newbury Park, CA: Sage.

Jerusalem, M., \& Mittag, W. (1995). Self-efficacy in life transitions. In A. Bandura (Ed.), Self-efficacy in changing societies (pp. 177-201). New York, NY: Cambridge University Press.

Johannesen, R.L. (1974). The functions of silence: A plea for communication research. Western Speech, 38, 25-35.

Johnson, J.P., Lenartowicz, T., \& Apud, S. (2006). Cross-cultural competence in international business: Towards a definition and model. Journal of International Business Studies, 37, $525-543$.

Joiner, T.E., \& Walker, R.L. (2002). Construct validity of a measure of acculturative stress in African Americans. Psychological Assessment, 14(4), 462-466. doi:10.1037/10403590.14.4.462

Kagan, H., \& Cohen, J. (1990). Cultural adjustment of international students. Psychological Science, 1(2), 133-137. doi:10.1111/j.1467-9280.1990.tb00082.x

Kang, S.-M. (2006). Measurement of acculturation, scale formats, and language competence: Their implications for adjustment. Journal of Cross-Cultural Psychology, 37(6), 669693. doi:10.1177/0022022106292077

Kang, S.-M., Shaver, P.R., Sue, S., Min, K.-H., \& Jing, H. (2003). Culture-specific patterns in the prediction of life satisfaction: Roles of emotion, relationship quality, and selfesteem. Personality and Social Psychology Bulletin, 29(12), 1596-1608. 
Kappe, R., \& Van der Flier, H. (2010). Using multiple and specific criteria to assess the predictive validity of the Big Five personality factors on academic performance. Journal of Research in Personality, 44, 142-145. doi:10.1016/j.jrp.2009.11.002

Kashima, E., \& Loh, E. (2006). International students' acculturation: Effects of international, conational, and local ties and need for closure. International Journal of Intercultural Relations, 30(4), 471-485. doi:10.1016/j.ijintrel.2005.12.003

Kasser, T., \& Ryan, R.M. (1996). Further examining the American dream: Differential correlates of intrinsic and extrinsic goals. Personality and Social Psychology Bulletin, $22,280-287$.

Katz, L. F., Hessler, D. M., \& Annest, A. (2007). Domestic violence, emotional competence, and child adjustment. Social Development, 16(3), 513-538. doi:10.1111/j.14679507.2007.00401.x

Kealey, D.J. (1989). A study of cross-cultural effectiveness: Theoretical issues, practical applications. International Journal of Intercultural Relations, 13(3), 387-428.

Kelley, C., \& Meyers, J.E. (1995). The Cross-Cultural Adaptability Inventory: Manual. Minneapolis, MN: Reid London House.

Kendon, A. (1967). Some functions of gaze direction in social interaction. Acta Psychologica, 23, 22-63.

Kendon, A. (1992). Some recent work from Italy on quotable gestures (“emblems"). Journal of Linguistic Anthropology, 2, 72-93.

Kendon, A. (1997). Gesture. Annual Review of Anthropology, 26, 109-128.

Kennedy, A. (2000). Singaporean sojourners: Meeting the demands of cross-cultural transitions. Unpublished doctoral dissertation. National University of Singapore.

Kenny, D.A., Korchmaros, J.D., \& Bolger, N. (2003). Lower level mediation in multilevel models. Psychological Methods, 8(2), 115-128. doi:10.1037/1082-989X.8.2.115

Kessler, R.C., Mickelson, K.D., \& Williams, D.R. (1999). The prevalence, distribution, and mental health correlates of perceived discrimination in the United States. Journal of Health and Social Behavior, 40(3), 208-230.

Kim, E. (2009). Beyond language barriers: Teaching self-efficacy among East Asian international teaching assistants. International Journal of Teaching and Learning in Higher Education, 21(2), 171-180.

Kim, M.S. (1995). Towards a theory of conversational restraints: Focusing on individuallevel dimensions of culture. In R.L. Wiseman (Ed.), Intercultural communication theory (pp. 148-169). Thousand Oaks, CA: Sage. 
Kim, O. (1999). Predictors of loneliness in elderly Korean immigrant women living in the United States of America. Journal of Advanced Nursing, 29(5), 1082-1088. doi:10.1046/j.1365-2648.1999.00993.x

Kim, S.Y., Wang, Y., Deng, S., Alvarez, R., \& Li, J. (2011). Accent, perpetual foreigner stereotype, and perceived discrimination as indirect links between English proficiency and depressive symptoms in Chinese American adolescents. Developmental Psychology, 47(1), 289-301. doi:10.1037/a0020712

Kim, Y.Y. (1991). Intercultural communication competence: A systems-theoretic view. In S. Ting-Toomey \& F. Korzenny (Eds.), Cross-cultural interpersonal communication (pp. 259-275). Newbury Park, CA: Sage Publications.

Kim, Y.Y. (1995). Interethnic communication: The context and the behavior. In S. Deetz (Ed.), Communication yearbook (pp. 511-538). Newbury Park, CA: Sage.

Kline, P. (1999). The handbook of psychological testing (2nd ed.). London: Routledge.

Kline, R.B. (2005). Principles and practice of structural equation modeling. New York, NY: Guilford Press.

Kline, R.B. (2010). Principles and practice of structural equation modeling (3rd ed.). New York, NY: The Guilford Press.

Klineberg, O., \& Hull, F.W. (1979). At a foreign university: An international study of adaptation and coping. New York, NY: Praeger Publishers.

Knapp, M.L., \& Hall, J.A. (2002). Nonverbal communication in human interaction. Belmont, CA: Wadsworth.

Kobrin, S.J. (1984). International expertise in American business. New York, NY: Institute of International Education.

Koestner, R., Lekes, N., Powers, T.A., \& Chicoine, E. (2002). Attaining personal goals: Selfconcordance plus implementation intentions equals success. Journal of Personality and Social Psychology, 83(1), 231-244. doi:10.1037/0022-3514.83.1.231

Korpela, M. (2011). Me, myself and I: Western lifestyle migrants in search of themselves in Varanasi, India. Recreation and Society in Africa, Asia and Latin America, 1(1), 53-73.

Kosic, A. (2006). Personality and individual factors in acculturation. In D.L. Sam \& J.W. Berry (Eds.), The Cambridge handbook of acculturation psychology (pp. 113-128). Cambridge: Cambridge University Press.

Kosic, A. (2004). Acculturation strategies, coping process and acculturative stress. Scandinavian Journal of Psychology, 45(4), 269-78. doi:10.1111/j.14679450.2004.00405.x 
Kraemer, H.C., Yesavage, J.A., Taylor, J.L., \& Kupfer, D (2000). How can we learn about developmental processes from cross-sectional studies, or can we? American Journal of Psychiatry, 157(2), 163-171. doi:10.1176/appi.ajp.157.2.163

Kurman, J., Eshel, Y., \& Sbeit, K. (2005). Acculturation attitudes, perceived attitudes of the majority, and adjustment of Israeli-Arab and Jewish-Ethiopian students to an Israeli university. The Journal of Social Psychology, 145(5), 593-612.

Kurman, J., \& Ronen-Eilon, C. (2004). Lack of knowledge of a culture's social axioms and adaptation difficulties among immigrants. Journal of Cross-Cultural Psychology, 35(2), 192-208. doi:10.1177/0022022103262244

Kus, L., \& Ward, C. (2009). Testing the concordance model of acculturation: Acculturation attitudes as determinants of immigrants' relations. Sixth Biennial Conference of the International Academy of Intercultural Research. Honolulu, HI.

LaFrance, M., \& Mayo, C. (1976). Racial differences in gaze behavior during conversations: Two systematic observational studies. Journal of Personality and Social Psychology, $33(5), 547-552$.

Lai, C.J. (2006). Sociocultural adaptation and intercultural sensitivity among international instructors of teaching English as a foreign language (TEFL) in universities and colleges in Taiwan (China). Unpublished doctoral dissertation. University of Minnesota.

Lalonde, R.N., \& Cameron, J.E. (1993). An intergroup perspective on immigrant acculturation with a focus on collective strategies. International Journal of Psychology, $28,57-74$.

Latham, G.P., Fay, C., \& Saari, L. (1979). The development of behavioral observation scales for appraising the performance of foremen. Personnel Psychology, 32, 299-311.

Lazarus, R., \& Folkman, S. (1984). Stress, appraisal, and coping. New York, NY: Springer.

Lee, D.L., \& Ahn, S. (2011). Discrimination against Latina/os: A meta-analysis of individuallevel resources and outcomes. The Counseling Psychologist, 40(1), 28-65. doi:10.1177/0011000011403326

Lee, E.S. (1966). A theory of migration. Demography, 3(1), 47-57.

Lee, R.M. (2005). Resilience against discrimination: Ethnic identity and other-group orientation as protective factors for Korean Americans. Journal of Counseling Psychology, 52(1), 36-44. doi:10.1037/0022-0167.52.1.36

Legido-Quigley, H., \& McKee, M. (2012). Health and social fields in the context of lifestyle migration. Health \& Place, 18(6), 1209-1216. doi:10.1016/j.healthplace.2012.08.005 
Leong, C.-H. (2007). Predictive validity of the Multicultural Personality Questionnaire: A longitudinal study on the sociopsychological adaptation of Asian undergraduates who took part in a study-abroad program. International Journal of Intercultural Relations, 31(5), 545-559. doi:10.1016/j.ijintrel.2007.01.004

Leong, C.-H., \& Ward, C. (2000). Identity conflict in sojourners. International Journal of Intercultural Relations, 24(6), 763-776. doi:10.1016/S0147-1767(00)00030-4

Leung, C. (2001). The psychological adaptation of overseas and migrant students in Australia. International Journal of Psychology, 36(4), 251-259.

Levenson, R.W., Ekman, P., \& Friesen, W.V. (1990). Voluntary facial action generates emotion-specific autonomic nervous system activity. Psychophysiology, 27, 363-384.

Lewthwaite, M. (1996). A study of international students' perspectives on cross-cultural adaptation. International Journal for the Advancement of Counselling, 19(2), 167-185. doi:10.1007/BF00114787

Li, A., \& Gasser, M. (2005). Predicting Asian international students' sociocultural adjustment: A test of two mediation models. International Journal of Intercultural Relations, 29(5), 561-576. doi:10.1016/j.ijintrel.2005.06.003

Liebkind, K. (2001). Acculturation. In R. Brown \& S.L. Gaertner (Eds.), Blackwell handbook of social psychology: Intergroup processes. Oxford: Blackwell.

Liebkind, K., Jasinskaja-Lahti, I., \& Solheim, E. (2004). Cultural identity, perceived discrimination, and parental support as determinants of immigrants' school adjustments: Vietnamese youth in Finland. Journal of Adolescent Research, 19(6), 635-656. doi:10.1177/0743558404269279

Lipsey, M.W., \& Wilson, D.B. (2001). Practical meta-analysis (Vol. 49). Thousand Oaks, CA: Sage.

Little, T.D., Lindenberger, U., \& Nesselroade, J.R. (1999). On selecting indicators for multivariate measurement and modeling with latent variables: When "good" indicators are bad and "bad" indicators are good. Psychological Methods, 4(2), 192-211. doi:10.1037/1082-989X.4.2.192

Locke, S., \& Feinsod, F. (1982). Psychological preparation for young adults traveling abroad. Adolescence, 17, 815-819.

Long, J.-H., Yan, W.-H., Yang, H.-D., \& Van Oudenhoven, J.P. (2009). Cross-cultural adaptation of Chinese students in the Netherlands. US-China Education Review, 6, 1-8.

Lucksted, A., Drapalski, A., Calmes, C., Forbes, C., DeForge, B., \& Boyd, J. (2011). Ending self-stigma: Pilot evaluation of a new intervention to reduce internalized stigma among 
people with mental illnesses. Psychiatric Rehabilitation Journal, 35(1), 51-54. doi:10.2975/35.1.2011.51.54

Lundmark, L. (2006). Mobility, migration and seasonal tourism employment: Evidence from Swedish mountain municipalities. Scandinavian Journal of Hospitality and Tourism, 6(3), 197-213. doi:10.1080/15022250600866282

Lynn, R. (1981). Cross-cultural differences in neuroticism, extraversion and psychoticism. In R. Lynn (Ed.), Dimensions of personality: Papers in honour of E.J. Eysenck (pp. 263286). Oxford: Pergamon.

Lysgaard, S. (1955). Adjustment in a foreign society: Norwegian Fulbright grantees visiting the United States. International Social Science Bulletin, 7, 45-51.

MacCallum, R.C., Widaman, K.F., Zhang, S., \& Hong, S. (1999). Sample size in factor analysis. Psychological Methods, 4(1), 84-99. doi:10.1037/1082-989X.4.1.84

MacKinnon, D.P. (2000). Contrasts in multiple mediator models. In J.S. Rose, L. Chassin, C.C. Presson, \& S.J. Sherman (Eds.), Multivariate applications in substance use research: New methods for new questions (pp. 141-160). Mahwah, NJ: Lawrence Erlbaum Associates Publishers.

Major, B., Quinton, W.J., \& McCoy, S.K. (2002). Antecedents and consequences of attributions to discrimination: Theoretical and empirical advances. In M.P. Zanna (Ed.), Advances in experimental social psychology (pp. 251-300). San Diego, CA: Academic Press.

Mak, A.S., \& Barker, M. (2004). A social cognitive learning program for facilitating intercultural relations. In Y. Kashima, Y. Endo, E.S. Kashima, C. Leung, \& J. McClure (Eds.), Progress in Asian social psychology, Vol. 4 (pp. 157-179). Seoul: KyoyookKwahak-Sa Publishing Co.

Mak, A.S., \& Buckingham, K. (2007). Beyond communication courses: Are there benefits in adding skills-based ExcelL ${ }^{\mathrm{TM}}$ sociocultural training? International Journal of Intercultural Relations, 31(3), 277-291. doi:10.1016/j.ijintrel.2006.02.002

Mak, A.S., \& Nesdale, D. (2001). Migrant distress: The role of perceived racial discrimination and coping resources. Journal of Applied Social Psychology, 31(12), 2632-2647. doi:10.1111/j.1559-1816.2001.tb00194.x

Mak, A.S., \& Tran, C. (2001). Big five personality and cultural relocation factors in Vietnamese Australian students' intercultural social self-efficacy. International Journal of Intercultural Relations, 25(2), 181-201. doi:10.1016/S0147-1767(00)00050-X 
Mak, A.S., Westwood, M.J., Barker, M.C., \& Ishiyama, F.I. (1998). The ExcelL Program for developing international students' sociocultural competencies. Journal of International Education, 9(1), 33-38.

Mallinckrodt, B., Abraham, W.T., Wei, M., \& Russell, D.W. (2006). Advances in testing the statistical significance of mediation effects. Journal of Counseling Psychology, 53(3), 372-378. doi:10.1037/0022-0167.53.3.372

Maretzki, T.W. (1969). Transcultural adjustment of Peace Corps volunteers. In J.C. Finney (Ed.), Culture change, mental health and poverty (pp. 203-221). Lexington, KT: University of Kentucky Press.

Markus, H.R., \& Kitayama, S. (1991). Culture and the self: Implications for cognition, emotion, and motivation. Psychological Review, 98(2), 224-253. doi:10.1037//0033295X.98.2.224

Marsella, A.J., \& Ring, E. (2003). Human migration and immigration. In L. Adler \& U. Gielen (Eds.), Migration: Immigrants and emigration in international perspective (pp. 3-22). Westport, CT: Greenwood Press.

Marsh, H.W., Hau, K.-T., Balla, J.R., \& Grayson, D. (1998). Is more ever too much? The number of indicators per factor in confirmatory factor analysis. Multivariate Behavioral Research, 33(2), 181-220. doi:10.1207/s15327906mbr3302_1

Martin, P.L. (1993). The immigration issue. In R. King (Ed.), The new geography of European migrants (pp. 1-16). London: Belhaven.

Masgoret, A.-M. (2006). Examining the role of language attitudes and motivation on the sociocultural adjustment and the job performance of sojourners in Spain. International Journal of Intercultural Relations, 30, 311-331.

Masgoret, A.-M., Bernaus, M., \& Gardner, R.C. (2000). A study of cross-cultural adaptation by English-speaking sojourners in Spain. Foreign Language Annals, 33(5), 548-558. doi:10.1111/j.1944-9720.2000.tb01998.x

Masgoret, A.-M., \& Gardner, R.C. (2003). Attitudes, motivation, and second-language learning: A meta-analysis of studies conducted by Gardner and associates. Language Learning, 53(1), 123-163. doi:10.1111/1467-9922.00212

Masgoret, A.-M., \& Ward, C. (2006). Culture learning approach to acculturation. In D.L. Sam \& J.W. Berry (Eds.), The Cambridge handbook of acculturation psychology. Cambridge: Cambridge University Press.

Massimini, F., \& Delle Fave, A. (2000). Individual development in a bio-cultural perspective. American Psychologist, 55(1), 24-33. doi:10.1037/0003-066X.55.1.24 
Matear, D.W. (2009). An examination of cognitive, cultural, and emotional intelligences, and motivation in the development of global transformational leadership skills. Unpublished doctoral dissertation. Capella University.

Matsumoto, D. (1989). Cultural influences on the perception of emotion. Journal of CrossCultural Psychology, 20(1), 92-105.

Matsumoto, D. (1990). Cultural similarities and differences in display rules. Motivation and Emotion, 14(3), 195-214.

Matsumoto, D., \& Ekman, P. (1989). American-Japanese cultural differences in intensity ratings of facial expressions of emotion. Motivation and Emotion, 13(2), 143-157. doi:10.1007/BF00992959

Matsumoto, D., Kasri, F., \& Kooken, K. (1999). American-Japanese cultural differences in judgments of expression intensity and subjective experience. Cognition and Emotion, $13,201-218$.

Matsumoto, D., Kudoh, T., Scherer, K., \& Wallbott, H. (1988). Antecedents of and reactions to emotions in the United States and Japan. Journal of Cross-Cultural Psychology, 19(3), 267-286. doi:10.1177/0022022188193001

Mayer, J.D., \& Salovey, P. (1993). The intelligence of emotional intelligence. Intelligence, 17(4), 433-442. doi:10.1016/0160-2896(93)90010-3

Mayer, J.D., \& Salovey, P. (1997). What is emotional intelligence? In P. Salovey \& D.J. Sluyter (Eds.), Emotional development and emotional intelligence (pp. 3-31). New York, NY: Basic Books.

Mayer, J.D., Salovey, P., \& Caruso, D.R. (2000). Models of emotional intelligence. In R.J. Sternberg (Ed.), Handbook of Intelligence. Cambridge: Cambridge University Press.

McCarthy, A., Lee, K., Itakura, S., \& Muir, D.W. (2006). Cultural display rules drive eye gaze during thinking. Journal of Cross-Cultural Psychology, 37(6), 717-722. doi:10.1177/0022022106292079

McCrae, R.R. (2002). NEO-PI-R data from 36 cultures. In R.R. McCrae \& J. Allik (Eds.), The five-factor model of personality across cultures (pp. 105-125). New York, NY: Kluwer Academic.

McCrae, R.R., Costa, P.T., \& Yik, M.S. (1996). Universal aspects of Chinese personality structure. In M.H. Bond (Ed.), The handbook of Chinese psychology (Vol. 88, pp. 189207). Hong Kong: Oxford University Press. doi:10.1037/0022-3514.88.3.547 
McDaniel, E., \& Anderson, P.A. (1998). International patterns of interpersonal tactile communication: A field study. Journal of Nonverbal Behaviour, 22(1), 59-75. doi:10.1023/A: 10229509743

Mclain, D.L. (1993). The Mstat-I: A new measure of an individual's tolerance for ambiguity. Educational and Psychological Measurement, 53(1), 183-189. doi:10.1177/0013164493053001020

McNair, D.M., Loor, M., \& Droppleman, L.F. (1981). Profile of Mood States. San Diego, CA: EdITS.

Mendenhall, M., \& Oddou, G. (1985). The dimensions of expatriate acculturation: A review. Academy of Management Review, 10(1), 39-47.

Mesoudi, A. (2009). How cultural evolutionary theory can inform social psychology and vice versa. Psychological Review, 116(4), 929-52. doi:10.1037/a0017062

Mesoudi, A. (2008). An experimental simulation of the "copy-successful-individuals" cultural learning strategy: Adaptive landscapes, producer-scrounger dynamics, and informational access costs. Evolution and Human Behavior, 29(5), 350-363. doi:10.1016/j.evolhumbehav.2008.04.005

Mischel, W. (1984). On the predictability of behavior and the structure of personality. In R.A. Zucker, J. Aronoff, \& A.I. Rabin (Eds.), Personality and the prediction of behavior (pp. 269-305). New York, NY: Academic Press.

Mischel, W., \& Shoda, Y. (1995). A cognitive-affective system theory of personality: Reconceptualizing situations, dispositions, dynamics, and invariance in personality structure. Psychological Review, 102(2), 246-268. doi:10.1037/0033-295X.102.2.246

Miyahira, K. (1991). Needs profiles of intercultural communication competence in JapaneseAmerican student dyads. The Conference on Communication in Japan and the United States. Fullerton, CA.

Mol, S.T., Born, M., \& Van der Molen, H.T. (2005). Developing criteria for expatriate effectiveness: Time to jump off the adjustment bandwagon. International Journal of Intercultural Relations, 29, 339-353.

Molinsky, A.L. (2007). Cross-cultural code-switching: The psychological challenges of adapting behavior in foreign cultural interactions. The Academy of Management Review, $32(2), 622-640$.

Molinsky, A.L., Krabbenhoft, M.A., Ambady, N., \& Choi, Y.S. (2005). Cracking the nonverbal code: Intercultural competence and gesture recognition across cultures. Journal of Cross-Cultural Psychology, 36(3), 380-395. doi:10.1177/0022022104273658 
Montreuil, A., \& Bourhis, R.Y. (2001). Majority acculturation orientations toward "valued" and "devalued" immigrants. Journal of Cross-Cultural Psychology, 32(6), 698-719. doi:10.1177/0022022101032006004

Moore, T.M. (2009). The sojourner's truth: Exploring bicultural identity as a predictor of assignment success in American expatriates. Unpublished doctoral dissertation. North Carolina State University.

Myrick, S.E., \& Martorell, G.A. (2011). Sticks and stones may break my bones: Protective factors for the effects of perceived discrimination on social competence in adolescence. Personal Relationships, 18(3), 487-501. doi:10.1111/j.1475-6811.2010.01320.x

Napier, N.K., \& Taylor, S. (1995). Western women working in Japan: Breaking corporate barriers. Westport, CT \& London: Quorum Press.

Nesdale, D., \& Mak, A.S. (2003). Ethnic identification, self-esteem and immigrant psychological health. International Journal of Intercultural Relations, 27(1), 23-40. doi:10.1016/S0147-1767(02)00062-7

Neto, F., \& Barros, J. (2000). Predictors of loneliness among adolescents from Portuguese immigrant families in Switzerland. Social Behavior and Personality: An International Journal, 28(2), 193-205. doi:10.2224/sbp.2000.28.2.193

Neto, F. (2006). Psycho-social predictors of perceived discrimination among adolescents of immigrant background: A Portuguese study. Journal of Ethnic and Migration Studies, 32(1), 89-109. doi:10.1080/13691830500335507

Noe, R.A. (1986). Trainee attributes and attitudes: Neglected influences on training effectiveness. Academy of Management Review, 11, 736-749.

Noels, K.A., Pelletier, L.G., Clément, R., \& Vallerand, R.J. (2000). Why are you learning a second language? Motivational orientations and Self-Determination Theory. Language Learning, 50(1), 57-85. doi:10.1111/0023-8333.00111

Noels, K.A., Pon, G., \& Clément, R. (1996). Language, identity, and adjustment: The role of linguistic self-confidence in the acculturation process. Journal of Language and Social Psychology, 15(3), 246-264.

Nowok, B., Van Ham, M., Findlay, A., \& Gayle, V. (2011). Does migration make you happy? A longitudinal study of internal migration and subjective well-being. IZA Discussion Paper No. 6140. Retrieved from http://ssrn.com/abstract=1968091

Nunnally, J. (1978). Psychometric theory. New York, NY: McGraw-Hill.

Oberg, K. (1960). Cultural shock: Adjustment to new cultural environments. Practical Anthropology, 7, 177-182. 
Oberoi, S.S., \& Lin, V. (2006). Brain drain of doctors from southern Africa: Brain gain for Australia. Australian Health Review, 30(1), 25-33. doi:10.1071/AH060025

Oguri, M., \& Gudykunst, W.B. (2002). The influence of self construals and communication styles on sojourners' psychological and sociocultural adjustment. International Journal of Intercultural Relations, 26, 577-593.

Ones, D.S., \& Viswesvaran, C. (1997). Personality determinants in the prediction of aspects of expatriate job success. In Z. Aycan (Ed.), New approaches to employee management, Vol. 4: Expatriate management: Theory and research (pp. 63-92). Greenwich, CT: JAI.

Ong, A.S.J., \& Ward, C. (2005). The construction and validation of a social support measure for sojourners: The Index of Sojourner Social Support (ISSS) Scale. Journal of CrossCultural Psychology, 36(6), 637-661. doi:10.1177/0022022105280508

Ono, M. (2008). Long-stay tourism and international retirement migration: Japanese retirees in Malaysia. Transnational Migration in East Asia, 77, 151-162.

Oppedal, B., Røysamb, E., \& Heyerdahl, S. (2005). Ethnic group, acculturation, and psychiatric problems in young immigrants. Journal of Child Psychology and Psychiatry, and Allied Disciplines, 46(6), 646-60. doi:10.1111/j.1469-7610.2004.00381.x

Oppedal, B., Røysamb, E., \& Sam, D.L. (2004). The effect of acculturation and social support on change in mental health among young immigrants. International Journal of Behavioral Development, 28(6), 481-494. doi:10.1080/01650250444000126

Ornelas, I.J., \& Perreira, K.M. (2011). The role of migration in the development of depressive symptoms among Latino immigrant parents in the USA. Social Science and Medicine, 73(8), 1169-1177. doi:10.1016/j.socscimed.2011.07.002

Osborne, J.W., \& Costello, A.B. (2004). Sample size and subject to item ratio in principal components analysis. Practical Assessment, Research and Evaluation, 9(11). Retrieved December 2, 2010 from http://PAREonline.net/getvn.asp?v=9\&n=11

Overton, R.C. (1998). A comparison of fixed-effects and mixed (random effects) models for meta-analysis tests of moderator variable effects, Psychological Methods, 3(3), 354379.

Padilla, A.M., \& Perez, W. (2003). Acculturation, social identity, and social cognition: A new perspective. Hispanic Journal of Behavioral Sciences, 25(1), 35-55. doi:10.1177/0739986303251694

Padilla, A.M., Wagatsuma, Y., \& Lindholm, K.J. (1985). Acculturation and personality as predictors of stress in Japanese and Japanese-Americans. The Journal of Social Psychology, 125(3), 295-305. 
Parker, B., \& McEvoy, G.M. (1993). Initial examination of a model of intercultural adjustment. International Journal of Intercultural Relations, 17(3), 355-379.

Pascoe, E.A., \& Smart Richman, L. (2009). Perceived discrimination and health: A metaanalytic review. Psychological Bulletin, 135(4), 531-54. doi:10.1037/a0016059

Pavot, W., \& Diener, E. (1993). Review of the Satisfaction with Life Scale. Psychological Assessment, 5(2), 164-172. doi:10.1037/1040-3590.5.2.164

Pavot, W., \& Diener, E. (2008). The Satisfaction with Life Scale and the emerging construct of life satisfaction. The Journal of Positive Psychology, 3(2), 137-152. doi:10.1080/17439760701756946

Pedersen, P., Draguns, J., Lonner, W.J., \& Trimble, J. (Eds.). (2007). Counseling across cultures. Thousand Oaks, CA: Sage.

Pelletier, L.G., Fortier, M.S., Vallerand, R.J., \& Brière, N.M. (2001). Associations among perceived autonomy support, forms of self-regulation, and persistence: A prospective study. Motivation and Emotion, 25(4), 279-306.

Pennebaker, J.W., Rimé, B., \& Blankenship, V. (1996). Stereotypes of northerners and southerners: A cross-cultural test of Montesque's hypothesis. Journal of Personality and Social Psychology, 70, 372-380.

Perrucci, R., \& Hu, H. (1995). Satisfaction with social and educational experiences among international graduate students. Research in Higher Education, 36(4), 491-508. doi:10.1007/BF02207908

Peterson, R.A., \& Brown, S.P. (2005). On the use of beta-coefficients in meta-analysis. Journal of Applied Psychology, 90(1), 175-181.

Pettigrew, T.F., \& Tropp, L.R. (2006). A meta-analytic test of intergroup contact theory. Journal of Personality and Social Psychology, 90(5), 751-83. doi:10.1037/00223514.90.5.751

Phinney, J.S., Horenczyk, G., Liebkind, K., \& Vedder, P. (2001). Ethnic identity, immigration, and well-being: An interactional perspective. Journal of Social Issues, 57(3), 493-510. doi:10.1111/0022-4537.00225

Phinney, J.S., Madden, T., \& Santos, L.J. (1998). Psychological variables as predictors of perceived ethnic discrimination among minority and immigrant adolescents. Journal of Applied Social Psychology, 28(11), 937-953. doi:10.1111/j.1559-1816.1998.tb01661.x

Piontkowski, U., Florack, A., Hoelker, P., \& Obdrzalek, P. (2000). Predicting acculturation attitudes of dominant and non-dominant groups. International Journal of Intercultural Relations, 24(1), 1-26. 
Podsakoff, P.M., MacKenzie, S.B., Lee, J.-Y., \& Podsakoff, N.P. (2003). Common method biases in behavioral research: A critical review of the literature and recommended remedies. The Journal of Applied Psychology, 88(5), 879-903. doi:10.1037/00219010.88.5.879

Ponterotto, J.G., Fuertes, J.N., \& Chen, E.C. (2000). Models of multicultural counseling. In S.D. Brown \& R.W. Lent (Eds.), Handbook of counseling psychology (3rd ed., pp. 639669). Hoboken, NJ: John Wiley \& Sons.

Poortinga, Y.H., Schoots, N.H., \& Van De Koppel, J.M. (1993). The understanding of Chinese and Kurdish emblematic gestures by Dutch subjects. International Journal of Psychology, 28, 41-44.

Pope-Davis, D.B., Breaux, C., \& Liu, W.M. (1997). A multicultural immersion experience: Filling a void in multicultural training. In D.B. Pope-Davis \& H.L.K. Coleman (Eds.), Multicultural counseling competencies: Assessment, education and training, and supervision. Multicultural aspects of counseling series, Vol. 7 (pp. 227-241). Thousand Oaks, CA: Sage Publications.

Porter, R.E., \& Samovar, L.A. (1998). Cultural influences on emotional expression: Implications for intercultural communication. In P.A. Andersen \& L.K. Guerrero (Eds.), Handbook of communication and emotion: Research, theory, applications, and contexts (pp. 451-472). San Diego, CA: Academic Press.

Portes, A. (1997). Immigration theory for a new century: Some problems and opportunities. International Migration Review, 31(4), 799-825.

Prelow, H.M., Weaver, S.R., \& Swenson, R.R. (2006). Competence, self-esteem, and coping efficacy as mediators of ecological risk and depressive symptoms in urban African American and European American youth. Journal of Youth and Adolescence, 35(4), 506-516. doi:10.1007/s10964-006-9068-Z

Pruitt, F.J. (1978). The adaptation of African students to American society. International Journal of Intercultural Relations, 2, 90-118.

Pulakos, E.D., Arad, S., Donovan, M.A., \& Plamondon, K.E. (2000). Adaptability in the workplace: Development of a taxonomy of adaptive performance. Journal of Applied Psychology, 85(4), 612-624. doi:10.1037//0021-9010.85.4.612

Quintrell, N., \& Westwood, M. (1994). The influence of a peer-pairing program on international students' first year experience and use of student services. Higher Education Research and Development, 13(1), 49-58. doi:10.1080/0729436940130105 
Radel, R., Sarrazin, P., \& Pelletier, L.G. (2009). Evidence of subliminally primed motivational orientations: The effects of unconscious motivational processes on the performance of a new motor task. Journal of Sport and Exercise Psychology, 31, 657674.

Rahman, O., \& Rollock, D. (2004). Acculturation, competence, and mental health among South Asian students in the United States. Journal of Multicultural Counseling and Development, 32(3), 130-142. doi:10.1002/j.2161-1912.2004.tb00366.x

Ratiu, I. (1983). Thinking internationally: A comparison of how international executives learn. International Studies of Management and Organization, 13(1/2), 139-150.

Ratner, C. (1997). Cultural psychology and qualitative methodology: Theoretical and empirical considerations. New York, NY: Plenum Publishing Corporation.

Raubenheimer, J. (2004). An item selection procedure to maximise scale reliability and validity. SA Journal of Industrial Psychology, 30(4), 59-64.

Redfield, R., Linton, R., \& Herskovits, M.J. (1936). Memorandum for the study of acculturation. American anthropologist, 38(1), 149-152.

Redmond, M.V., \& Bunyi, J.M. (1993). The relationship of intercultural communication competence with stress and the handling of stress as reported by international students. International Journal of Intercultural Relations, 17(2), 235-254. doi:10.1016/01471767(93)90027-6

Reis, H.T., \& Judd, C.M. (Eds.). (2000). Handbook of research methods in social and personality psychology. New York, NY: Cambridge University Press.

Reis, H.T., Sheldon, K.M., Gable, S.L., Roscoe, J., \& Ryan, R.M. (2000). Daily well-being: The role of autonomy, competence, and relatedness. Personality and Social Psychology Bulletin, 26(4), 419-435. doi:10.1177/0146167200266002

Revollo, H.-W., Qureshi, A., Collazos, F., Valero, S., \& Casas, M. (2011). Acculturative stress as a risk factor of depression and anxiety in the Latin American immigrant population. International Review of Psychiatry, 23(1), 84-92. doi:10.3109/09540261.2010.545988

Richardson, D.B., \& Rullo, V. (1992). Going global: Are you ready for an overseas assignment? Management Accounting, 73(12), 31-39.

Richerson, P.J., \& Boyd, R. (2005). Not by genes alone. Chicago, IL: University of Chicago Press.

Richmond, A.H. (1993). Reactive migration: Sociological perspectives on refugee movements. Journal of Refugee Studies, 6(1), 7-24. doi:10.1093/jrs/6.1.7 
Riggio, R.E. (1986). Assessment of basic social skills. Journal of Personality and Social Psychology, 51(3), 649-660. doi:10.1037//0022-3514.51.3.649

Robinson, W.S. (1950). Ecological correlations and the behavior of individuals. American Sociological Review, 15, 351-357.

Rogers, A.R. (1988). Does biology constrain culture? American Anthropologist, 90, 819-831.

Rohrlich, B.F., \& Martin, J.N. (1991). Host country and reentry adjustment of student sojourners. International Journal of Intercultural Relations, 15, 163-182.

Rokeach, M. (1973). The nature of human values. New York, NY: Free Press.

Rosenthal, R. (1984). Meta-analytic procedures for social research. Beverly Hills, CA: Sage.

Rosenthal, R., \& DiMatteo, M.R. (2001). Meta-analysis: Recent developments in quantitative methods for literature reviews, 52, 59-82.

Ruben, B.D. (1976). Assessing communication competence for intercultural adaptation. Group and Organization Studies, 1, 334-354.

Ruben, B.D. (1989). The study of cross-cultural competence: Traditions and contemporary issues. International Journal of Intercultural Relations, 13(3), 229-240. doi:10.1016/0147-1767(89)90011-4

Ruben, B.D., \& Kealey, D.J. (1979). Behavioral assessment of communication competency and the prediction of cross-cultural adaptation. International Journal of Intercultural Relations, 3(1), 15-47.

Rudmin, F.W. (2003). Critical history of the acculturation psychology of assimilation, separation, integration, and marginalization. Review of General Psychology, 7(1), 3-37. doi:10.1037/1089-2680.7.1.3

Rudmin, F.W. (2009). Constructs, measurements and models of acculturation and acculturative stress. International Journal of Intercultural Relations, 33, 106-123.

Rudmin, F.W., \& Ahmadzadeh, V. (2001). Psychometric critique of acculturation psychology: The case of Iranian migrants in Norway. Scandinavian Journal of Psychology, 42, 41-56.

Rule, N.O., Ambady, N., Adams, R.B., \& Macrae, C.N. (2008). Facets of the face: Accuracy and awareness in the perception and categorization of male sexual orientation. Journal of Personality and Social Psychology, 95, 1019-1028.

Rumbaut, R.G. (1991). The agony of exile: A study of the migration and adaptation of Indochinese refugee adults and children. In L. Frederik, J. Aheam, \& J.L. Athey (Eds.), Refugee children: Theory, research, and services (pp. 53-91). Baltimore, MD: Johns Hopkins University Press. 
Rummel, R.J. (1970). Applied factor analysis (p. 617). Evanston, IL: Northwestern University Press.

Runions, K., Priest, N., \& Dandy, J. (2011). Discrimination and psychological adjustment amongst Australian children from middle-eastern and asian backgrounds. The Australian Community Psychologist, 23(1), 23-33.

Ryan, R.M., \& Connell, J.P. (1989). Perceived locus of causality and internalization: Examining reasons for acting in two domains. Journal of Personality and Social Psychology, 57(5), 749-761. doi:10.1037/0022-3514.57.5.749

Ryan, R.M., \& Deci, E.L. (2000). Self-determination theory and the facilitation of intrinsic motivation, social development, and well-being. American Psychologist, 5(1), 68-78.

Ryan, R.M., \& Deci, E.L. (2001). Self-determination theory and the facilitation of intrinsic motivation, social development, and well-being. American Psychologist, 5(1), 68-78.

Ryan, R.M., Deci, E.L., \& Grolnick, W.S. (1995). Autonomy, relatedness, and the self: Their relation to development and psychopathology. In D. Cicchetti \& D.J. Cohen (Eds.), Developmental psychopathology: Theory and methods (pp. 618-655). New York, NY: Wiley.

Sabatier, C., \& Berry, J.W. (2008). The role of family acculturation, parental style, and perceived discrimination in the adaptation of second-generation immigrant youth in France and Canada. European Journal of Developmental Psychology, 5(2), 159-185. doi:10.1080/17405620701608739

Safadi, M., \& Valentine, C.A. (1988). Emblematic gestures among Hebrew speakers in Israel. International Journal of Intercultural Relations, 4, 327-361.

Sam, D.L. (2001). Satisfaction with life among international students: An exploratory study. Social Indicators Research, 53(3), 315-337. doi:10.1023/A:1007108614571

Sam, D.L. (2006). Acculturation: Conceptual background and core components. In D.L. Sam \& J.W. Berry (Eds.), The Cambridge handbook of acculturation psychology (pp. 1126). Cambridge: Cambridge University Press.

Sam, D.L., Vedder, P., Ward, C., \& Horenczyk, G. (2006). Psychological and sociocultural adaptation of immigrant youth. In J.W. Berry, J.S. Phinney, D.L. Sam, \& P. Vedder (Eds.), Immigrant youth in cultural transition: Acculturation, identity, and adaptation across national contexts (pp. 117-141). Mahwah, NJ: Lawrence Erlbaum Associates, Inc.

Schmitz, P.G. (2001). Psychological aspects of immigration. In L. Adler \& U. Gielen (Eds.), Cross-cultural topics in psychology (pp. 229-243). Westport, CT: Praeger. 
Schupe, E.I. (2007). Clashing cultures: A model of international student conflict. Journal of Cross-Cultural Psychology, 38(6), 750-771. doi:10.1177/0022022107308996

Schwartz, S.H. (1992). Universals in the content and structure of values: Theoretical advances and empirical tests in 20 countries. Advances in experimental social psychology. San Diego, CA: Academic Press.

Schwartz, S.H. (1994). Beyond individualism/collectivism: New dimensions of values. In U. Kim, H.C. Triandis, C. Kagitçibaşi, S.C. Choi, \& G. Yoon (Eds.), Individualism and collectivism: Theory application and methods. Newbury Park, CA: Sage.

Schwartz, S.H., Roccas, S., \& Horenczyk, G. (2000). Acculturation discrepancies and wellbeing: The moderating role of conformity. European Journal of Social Psychology, 30, $323-334$.

Searle, W., \& Ward, C. (1990). The prediction of psychological and sociocultural adjustment during cross-cultural transitions. International Journal of Intercultural Relations, 14, $449-464$.

Selmer, J. (2005). Cross-cultural training and expatriate adjustment in China: Western joint venture managers. Personnel Review, 34(1), 68-84. doi:10.1108/00483480510571888

Selmer, J., \& Leung, A.S.M. (2003). Expatriate career intentions of women on foreign assignments and their adjustment. Journal of Managerial Psychology, 18(3), 244-258. doi:10.1108/02683940310465252

Shaffer, J.A., \& Postlethwaite, B.E. (2012). A matter of context: A meta-analytic investigation of the relative validity of contextualized and non-contextualized personality measures. Personnel Psychology, 65(3), 445-493. doi:10.1111/j.17446570.2012.01250.x

Shaffer, M.A., Harrison, D.A., \& Gilley, K.M. (1999). Dimensions, determinants, and differences in the expatriate adjustment process. Journal of International Business Studies, 30(3), 557-581.

Sheldon, K.M., \& Elliot, A.J. (1999). Goal striving, need satisfaction, and longitudinal wellbeing: The self-concordance model. Journal of Personality and Social Psychology, 76(3), 482-497. doi:10.1037/0022-3514.76.3.482

Shrout, P.E., \& Bolger, N. (2002). Mediation in experimental and nonexperimental studies: New procedures and recommendations. Psychological Methods, 7(4), 422-445. doi:10.1037/1082-989X.7.4.422

Sinangil, H.K., \& Ones, D.S. (1997). Empirical investigations of the host country perspective in expatriate management. In Z. Aycan \& D.M. Sanders (Eds.), New approaches to 
employee management: Vol. 4. Expatriate management: Theory and research. Greenwich, CT: JAI Press.

Sinha, D. (1987). Psychology in India: A historical perspective. In G.H. Blowers \& A.M. Turtle (Eds.), Psychology moving east: Status of western psychology in Asia and Oceania (pp. 39-52). Boulder, CO: Westview.

Smith, C.A., Dobbins, C.J., \& Wallston, K.A. (1991). The mediational role of perceived competence in psychological adjustment to rheumatoid arthritis. Journal of Applied Social Psychology, 21(15), 1218-1244. doi:10.1111/j.1559-1816.1991.tb00467.x

Smith, P.B., Bond, M.H., \& Kagitçibaşi, C. (2006). Understanding social psychology across cultures: Living and working in a changing world. London: Sage Publications, Ltd.

Sneed, C.D. (2002). Correlates and implications for agreeableness in children. The Journal of Psychology, 136(1), 59-67. doi:10.1080/00223980209604138

Sodowsky, G.R., \& Lai, E.W.M. (1997). Asian immigrant variables and structural models of cross-cultural distress. In A. Booth, A. Crouter, \& N.S. Landale (Eds.), Immigration and the family: Research and policy on U.S. immigrants (pp. 211-234). Hillsdale, NJ: Lawrence Erlbaum Associates, Inc.

Stahl, G.K., \& Caligiuri, P. (2005). The effectiveness of expatriate coping strategies: The moderating role of cultural distance, position level, and time on the international assignment. The Journal of Applied Psychology, 90(4), 603-615. doi:10.1037/00219010.90.4.603

Stathi, S., Tsantila, K., \& Crisp, R.J. (2012). Imagining intergroup contact can combat mental health stigma by reducing anxiety, avoidance and negative stereotyping. The Journal of Social Psychology, 152(6), 746-757. doi:10.1080/00224545.2012.697080

Statistics New Zealand. (2010). International travel and migration: September 2011.

Retrieved from www.stats.govt.nz/browse_for_stats/population/Migration/ IntTravelAndMigration_HOTPSep11.aspx.

Stening, B.W. (1979). Problems in cross-cultural contact: A literature review. International Journal of Intercultural Relations, 3(3), 269-313. doi:10.1016/0147-1767(79)90016-6

Stening, B.W., \& Hammer, M.R. (1992). Cultural baggage and the adaption of expatriate American and Japanese managers. Management International Review, 32(1), 77-89.

Stevens, J.P. (1992). Applied multivariate statistics for the social sciences (2nd ed.). Hillsdale, NJ: Erlbaum. 
Stone, A.A., Turkkan, J.S., Bachrach, C.A., Jobe, J.B., Kurtzman, H.S., \& Cain, V.S. (2000). The science of self-report: Implications for research and practice. Mahwah, NJ: Lawrence Erlbaum Associates, Inc.

Stone Feinstein, B.E., \& Ward, C. (1990). Loneliness and psychological adjustment of sojourners: New perspectives on culture shock. In D.M. Keats, D. Munro, \& L. Mann (Eds.), Heterogeneity in cross-cultural psychology (pp. 537-547). Lisse: Swets \& Zeitlinger.

Stone, R. (1991). Expatriate selection and failure. Human Resource Planning, 14, 9-18.

Strand, P.S., Pula, K., Parks, C.D., \& Cerna, S. (2011). Shyness-anxiousness and receptive language skills development in Spanish- and English-speaking preschoolers. Journal of Applied Developmental Psychology, 32(6), 363-368. doi:10.1016/j.appdev.2011.06.002

Stuart, J.E. (2012). Pathways to positive development for Muslim immigrant youth in Western contexts. Unpublished doctoral dissertation. Victoria University of Wellington.

Suanet, I., \& Van de Vijver, F.J.R. (2009). Perceived cultural distance and acculturation among exchange students in Russia. Journal of Community and Applied Social Psychology, 19(3), 182-197. doi:10.1002/casp.989

Sue, D.W., Arrondondo, P., \& McDavis, R.J. (1992). Multicultural counseling competencies and standards: A call to the profession. Journal of Counseling and Development, 70, $477-486$.

Sue, D.W., \& Sue, D. (1977). Barriers to effective cross-cultural counseling. Journal of Counseling Psychology, 24(5), 420-429.

Suess, D. (1978). I can read with my eyes shut. New York, NY: Random House, Inc.

Sussman, N.M., \& Rosenfield, H.M. (1982). Influence of culture, language and sex on conversational distance. Journal of Personality and Social Psychology, 42, 66-74.

Swagler, M.A., \& Jome, L.M. (2005). The effects of personality and acculturation on the adjustment of North American sojourners in Taiwan. Journal of Counseling Psychology, 52(4), 527-536.

Swami, V. (2008). Predictors of sociocultural adjustment among sojourning Malaysian students in Britain. International Journal of Psychology, 44(4), 266-273. doi:10.1080/00207590801888745

Swami, V., Arteche, A., Chamorro-Premuzic, T., \& Furnham, A. (2010). Sociocultural adjustment among sojourning Malaysian students in Britain: A replication and path analytic extension. Social Psychiatry and Psychiatric Epidemiology, 45(1), 57-65. doi:10.1007/s00127-009-0042-4 
Swenson, L.L. (1980). Theories of learning. Belmont, CA: Wadsworth.

Tabachnick, B.G., \& Fidell, L.S. (2001). Using multivariate statistics (4th ed.,). Boston, MA: Allyn \& Bacon.

Tabor, A. S., \& Milfont, T. L. (2012). Family social support during the predeparture period: The experience of British migrants. International Journal of Psychology. doi:10.1080/00207594.2011.634008

Taft, R. (1977). Coping with unfamiliar cultures. In N. Warren (Ed.), (Vol. 1). London: Academic Press.

Tajfel, H. (1981). Human groups and social categories. Cambridge: Cambridge University Press.

Tajfel, H., \& Turner, J. (1979). An integrative theory of intergroup conflict. In W.G. Austin \& S. Worchel (Eds.), Monterey, CA: Brooks-Cole.

Tajfel, H., \& Turner, J. (1986). The social identity theory of intergroup behavior. In S. Worchel \& W.G. Austin (Eds.), Psychology of intergroup relations (pp. 7-24). Chicago, IL: Nelson-Hall.

Takeuchi, R., Yun, S., \& Tesluk, P.E. (2002). An examination of crossover and spillover effects of spousal and expatriate cross-cultural adjustment on expatriate outcomes. Journal of Applied Psychology, 87(4), 655-666. doi:10.1037/0021-9010.87.4.655

Tams, S. (2008). Constructing self-efficacy at work: A person-centered perspective. Personnel Review, 37, 165-183. doi:10.1108/00483480810850524

Tan, J.-S., \& Chua, R.Y.-J. (2003). Training and developing cultural intelligence. In P.C. Earley \& S. Ang (Eds.), Cultural intelligence: Individual interactions across cultures (pp. 258-303). Palo Alto, CA: Stanford University Press.

Tartakovsky, E., \& Schwartz, S. (2001). Motivation for emigration, values, wellbeing, and identification among young Russian Jews. International Journal of Psychology, 36(2), 88-99. doi:10.1080/00207590042000100

Taylor, D.M., \& Moghaddam, F.M. (1994). Theories of intergroup relations. Westport, CT: Praeger.

Templer, K.J., Tay, C., \& Chandrasekar, N.A. (2006). Motivational cultural intelligence, realistic job preview, realistic living conditions preview, and cross-cultural adjustment. Group and Organization Management, 31(1), 154-173. doi:10.1177/1059601105275293

Teodorescu, D.-S., Heir, T., Hauff, E., Wentzel-Larsen, T., \& Lien, L. (2012). Mental health problems and post-migration stress among multi-traumatized refugees attending 
outpatient clinics upon resettlement to Norway. Scandinavian Journal of Psychology, 53(4), 316-32. doi:10.1111/j.1467-9450.2012.00954.x

Tett, R.P., Jackson, D.N., \& Rothstein, M. (1991). Personality measures as predictors of job performance: A meta-analytic review. Personnel Psychology, 44(4), 703-742.

Ting-Toomey, S. (1988). Intercultural conflicts: A face-negotiation theory. In Y.Y. Kim \& W.B. Gudykunst (Eds.), Theories in intercultural communication. Newbury Park, CA: Sage.

Ting-Toomey, S., \& Chung, L. (1996). Cross-cultural interpersonal communication: Theoretical trends and research directions. In Y.Y. Kim \& W.B. Gudykunst (Eds.), Cross-cultural adaptation: Current approaches (pp. 237-261). Newbury Park, CA: Sage.

Ting-Toomey, S., \& Kurogi, A. (1998). Facework competence in intercultural conflict: An updated Face-Negotiation Theory. International Journal of Intercultural Relations, 22(2), 187-225.

Tinsley, H.E., \& Tinsley, D.J. (1987). Uses of factor analysis in counseling psychology research. Journal of Counseling Psychology, 34(4), 414-424. doi:10.1037/00220167.34.4.414

Torbiorn, I. (1982). Living abroad. New York, NY: Wiley.

Torres, L., \& Rollock, D. (2007). Acculturation and depression among Hispanics: The moderating effect of intercultural competence. Cultural Diversity and Ethnic Minority Psychology, 13(1), 10-17. doi:10.1037/1099-9809.13.1.10

Townsend, P., \& Wan, C. (2007). The impact of multicultural experience in the development of socio-cultural adaptation for international business students. International Journal of Educational Management, 21(3), 194-212. doi:10.1108/09513540710738656

Triandis, H.C. (1995). Individualism and collectivism. Boulder, CO: Westview Press.

Trice, A.G. (2004). Mixing it up: International graduate students' social interactions with American students. Journal of College Student Development, 45(6), 671-687. doi:10.1353/csd.2004.0074

Tropp, L.R., \& Pettigrew, T.F. (2005). Differential relationships between intergroup contact and affective and cognitive dimensions of prejudice. Personality and Social Psychology Bulletin, 31(8), 1145-1158. doi:10.1177/0146167205274854

Trower, P., Bryant, B., \& Argyle, M. (1978). Social skills and mental health. London: Methuen \& Co. Ltd. 
Tsang, E.W.K. (2001). Adjustment of mainland Chinese academics and students to Singapore. International Journal of Intercultural Relations, 25(4), 347-372. doi:10.1016/S0147-1767(01)00010-4

Tung, R.L. (1983). Selection and training procedures of U.S., European, and Japanese multinationals. The International Executive, 25(2), 5-7. doi:10.1002/tie.5060250204

Turner, J.C. (1999). Some current issues in research on social identity and self-categorization theories. In N. Ellemers, R. Spears, \& B. Doosie (Eds.), Social identity: Context, commitment, content. Oxford: Blackwell.

Ullman, C., \& Tatar, M. (2001). Psychological adjustment among Israeli adolescent immigrants: A report on life satisfaction, self-concept, and self-esteem. Journal of Youth and Adolescence, 30(4), 449-463. doi:10.1023/A:1010445200081

Ullman, J.B. (1996). Structural equation modeling. In B. G. Tabachnick \& L. S. Fidell (Eds.), Using multivariate statistics (pp. 653-771). New York, NY: Harper Collins College.

United Nations, Department of Economic and Social Affairs. (2011). International migration report 2009: A global assessment. Trends in international migrant stock: The 2008 revision.

Vallerand, R.J., \& Bissonnette, R. (1992). Intrinsic, extrinsic, and amotivational styles as predictors of behavior: A prospective study. Journal of Personality, 60(3), 599-620. doi:10.1111/j.1467-6494.1992.tb00922.x

Vallerand, R.J., Fortier, M.S., \& Guay, F. (1997). Self-determination and persistence in a real-life setting: Toward a motivational model of high school dropout. Journal of Personality and Social Psychology, 72(5), 1161-1176. doi:10.1037/00223514.72.5.1161

Van der Zee, K.I., \& Van Oudenhoven, J.P. (2000). The Multicultural Personality Questionnaire: A multidimensional instrument of multicultural effectiveness. European Journal of Personality, 14(4), 291-309. doi:http://dx.doi.org/10.1002/10990984(200007/08)14:4\%3C291::AID-PER377\%3E3.0.CO;2-6

Van der Zee, K.I., \& Van Oudenhoven, J.P. (2001). The Multicultural Personality Questionnaire: Reliability and validity of self- and other ratings of multicultural effectiveness. Journal of Research in Personality, 35(3), 278-288. doi:10.1006/jrpe.2001.2320

Van Dyne, L., Ang, S., \& Koh, C. (2008). Development and validation of the CQS. In S. Ang \& L. Van Dyne (Eds.), Handbook of cultural intelligence: Theory, measurement and applications (pp. 16-38). New York, NY: Sharpe. 
Van Dyne, L., Ang, S., Ng, K.Y., Rockstuhl, T., Tan, M.L., \& Koh, C. (2012). Subdimensions of the four factor model of cultural intelligence: Expanding the conceptualization and measurement of cultural intelligence. Social and Personality Psychology Compass, 6(4), 295-313. doi:10.1111/j.1751-9004.2012.00429.x

Van Oudenhoven, J., \& Van der Zee, K. (2002). Predicting multicultural effectiveness of international students: The Multicultural Personality Questionnaire. International Journal of Intercultural Relations, 26(6), 679-694. doi:10.1016/S0147-1767(02)00041$\mathrm{X}$

Van Selm, K., Sam, D.L., \& Van Oudenhoven, J.P. (1997). Life satisfaction and competence of Bosnian refugees in Norway. Scandinavian Journal of Psychology, 31, 143-149.

Van Tubergen, F., Maas, I., \& Flap, H. (2004). The economic incorporation of immigrants in 18 Western societies: Origin, destination, and community effects. American Sociological Review, 69(5), 704-727. doi:10.1177/000312240406900505

Vansteenkiste, M., Lens, W., Bart Soenens, B., \& Luyckx, K. (2006). Autonomy and relatedness among Chinese sojourners and applicants: Conflictual or independent predictors of well-being and adjustment? Motivation and Emotion, 30(4), 273-282.

Vansteenkiste, M., Smeets, S., Soenens, B., Lens, W., Matos, L., \& Deci, E.L. (2010). Autonomous and controlled regulation of performance-approach goals: Their relations to perfectionism and educational outcomes. Motivation and Emotion, 34(4), 333-353. doi:10.1007/s11031-010-9188-3

Vedadi, A., Kheiri, B., \& Abbasalizadeh, M. (2010). The relationship between cultural intelligence and achievement: A case study in an Iranian company. Iranian Journal of Management Studies, 3(3), 25-40.

Vedder, P.H., Sam, D., \& Liebkind, K. (2007). The acculturation and adaptation of Turkish adolescents in North-Western Europe. Applied Developmental Science, 11(3), 126-136. doi:10.1080/10888690701454617

Velicer, W.F., Eaton, C.A., \& Fava, J.L. (2000). Construct explication through factor or component analysis: A review and evaluation of alternative procedures for determining the number of factors or components. In R.D. Goffin \& E. Helmes (Eds.), Problems and solutions in human assessment: Honoring Douglas N. Jackson at seventy (pp. 41-71). Boston: Kluwer Academic Publishers.

Velicer, W.F., \& Fava, J.L. (1998). Effects of variable and subject sampling on factor pattern recovery. Psychological Methods, 3(2), 231-251. doi:10.1037/1082-989X.3.2.231 
Viechtbauer, W. (2007). Accounting for heterogeneity via random-effects models and moderator analyses in meta-analysis. Zeitschrift für Psychologie/Journal of Psychology, 215(2), 104-121.

Wang, C.-C. D., \& Mallinckrodt, B. (2006). Acculturation, attachment, and psychosocial adjustment of Chinese/Taiwanese international students. Journal of Counseling Psychology, 53(4), 422-433. doi:10.1037/0022-0167.53.4.422

Ward, C. (1996). Acculturation. In D. Landis \& R. Bhagat (Eds.), (pp. 124-147). Thousand Oaks, CA: Sage Publications.

Ward, C. (2001a). Culture learning. In C. Ward, S. Bochner, \& A. Furnham (Eds.), The Psychology of Culture Shock (2nd ed., pp. 51-69). London: Routledge.

Ward, C. (2001b). The ABCs of acculturation. In D. Matsumoto (Ed.), The handbook of culture and psychology (pp. 411-445). New York, NY: Oxford University Press.

Ward, C. (2004). Psychological theories of culture contact and their implications for intercultural training. In D. Landis, J. Bennet, \& M. Bennet (Eds.), Handbook of intercultural training (Vol. 3, pp. 185-216). Thousand Oaks, CA: Sage.

Ward, C. (2012). Facing challenges and continuing a fine tradition. International Journal of Intercultural Relations, 36, 1-2. doi:10.1016/j.ijintrel.2012.01.003

Ward C., Berno T., \& Main A. (2002) Can the Cross-cultural Adaptability Inventory predict sojourner adjustment? In P. Boski, F.J.R. van de Vijver, \& A.M. Chodnicka (Eds.), New directions in cross-cultural psychology (409-423). Warsaw: Polish Psychological Association.

Ward, C., Bochner, S., \& Furnham, A. (2001). The psychology of culture shock. East Sussex: Routledge.

Ward, C., \& Chang, W.C. (1997). "Cultural fit": A new perspective on personality and sojourner adjustment. International Journal of Intercultural Relations, 21(4), 525-533. doi:10.1016/S0147-1767(97)00023-0

Ward, C., \& Fischer, R. (2008). Personality, cultural intelligence, and cross-cultural adaptation. In S. Ang \& L. van Dyne (Eds.), Handbook of cultural intelligence: Theory, measurement, and applications (pp. 159-173). New York, NY: M.E. Sharpe, Inc.

Ward, C., Fischer, R., Lam, F.S.Z., \& Hall, L. (2008). The convergent, discriminant, and incremental validity of scores on a self-report measure of cultural intelligence. Educational and Psychological Measurement, 69(1), 85-105. doi:10.1177/0013164408322001 
Ward, C., Fox, S., Wilson, J., Stuart, J., \& Kus, L. (2010). Contextual influences on acculturation processes: The roles of family, community and society. Psychological Studies, 55(1), 26-34. doi:10.1007/s12646-010-0003-8

Ward, C., \& Kennedy, A. (1992a). Acculturation and cross-cultural adaptation of British residents in Hong Kong. The Journal of Social Psychology, 113(3), 395-397.

Ward, C., \& Kennedy, A. (1992b). Locus of control, mood disturbance, and social difficulty during cross-cultural transitions. International Journal of Intercultural Relations, 16(2), $175-194$.

Ward, C., \& Kennedy, A. (1993a). Psychological and socio-cultural adjustment during crosscultural transitions: A comparison of secondary students overseas and at home. International Journal of Psychology, 28(2), 129-147.

Ward, C., \& Kennedy, A. (1993b). Where's the culture in cross-cultural transition? Comparative studies of sojourner adjustment. Journal of Cross-Cultural Psychology, 24(2), 221-249. doi:10.1177/0022022193242006

Ward, C., \& Kennedy, A. (1994). Acculturation strategies, psychological adjustment, and sociocultural competence during cross-cultural transitions. International Journal of Intercultural Relations, 18(3), 329-343.

Ward, C., \& Kennedy, A. (1995). The relationship between psychological and sociocultural dimensions of cross-cultural adjustment. In J. Pandey, D.P.S. Bhawuk, \& D. Sinha (Eds.), Asian contributions to cross-cultural adjustment (pp. 178-295). New Delhi: Sage.

Ward, C., \& Kennedy, A. (1996a). Crossing cultures: The relationship between psychological and sociocultural dimensions of cross-cultural adjustment. In J. Pandey, D. Sinha, \& D. Bhawuk (Eds.), Asian contributions to cross-cultural psychology (pp. 289-306). London: Sage Publications Ltd.

Ward, C., \& Kennedy, A. (1996b). Before and after cross-cultural transition: A study of New Zealand volunteers on field assignments. In H. Grad, A. Blanco, \& J. Georgas (Eds.), Key issues in cross-cultural psychology: Selected papers from the Twelfth International Congress of the International Association for Cross-Cultural Psychology (pp. 138-154).

Ward, C., \& Kennedy, A. (1999). The measurement of sociocultural adaptation. International Journal of Intercultural Relations, 23(4), 659-677.

Ward, C., \& Leong, C.-H. (2006). Intercultural relations in plural societies: Theory, research and application. In D.L. Sam \& J.W. Berry (Eds.), Cambridge handbook of acculturation psychology. Cambridge: Cambridge University Press. 
Ward, C., Leong, C.-H., \& Low, M. (2004). Personality and sojourner adjustment: An exploration of the Big Five and the cultural fit proposition. Journal of Cross-Cultural Psychology, 35(2), 137-151. doi:10.1177/0022022103260719

Ward, C., \& Masgoret, A.-M. (2004). The experiences of international students in New Zealand: Report on the results of the national survey. Prepared for the New Zealand Ministry of Education. (p. 161). Retrieved from http://mudface.net/IEC_NewZealand InternationalStudentExperience.pdf

Ward, C., Okura, Y., Kennedy, A., \& Kojima, T. (1998). The u-curve on trial: A longitudinal study of psychological and sociocultural adjustment during cross-cultural transition. International Journal of Intercultural Relations, 22(3), 277-291. doi:10.1016/S01471767(98)00008-X

Ward, C., \& Rana-Deuba, A. (1999). Acculturation and adaptation revisited. Journal of Cross-Cultural Psychology, 30(4), 422.

Ward, C., \& Searle, W. (1991). The impact of value discrepancies and cultural identity on psychological and sociocultural adjustment of sojourners. International Journal of Intercultural Relations, 15, 209-225.

Ward, C., Stuart, J., \& Kus, L. (2011). The construction and validation of a measure of ethnocultural identity conflict. Journal of Personality Assessment, 93, 462-473.

Ward, C., Wilson, J., \& Fischer, R. (2011). Assessing the predictive validity of cultural intelligence over time. Personality and Individual Differences, 51(2), 138-142. doi:10.1016/j.paid.2011.03.032

Watkins, M.W. (2000). Monte Carlo PCA for parallel analysis. State College, PA: Ed \& Psych Associates.

Wei, M., Heppner, P.P., Ku, T.-Y., \& Liao, K.Y.-H. (2010). Racial discrimination stress, coping, and depressive symptoms among Asian Americans: A moderation analysis. Asian American Journal of Psychology, 1(2), 136-150. doi:10.1037/a0020157

Williams, D.R., Spencer, M.S., \& Jackson, J.S. (1999). Race, stress, and physical health: The role of group identity. In R.J. Contrada \& R.D. Ashmore (Eds.), Self, social identity, and physical health: Interdisciplinary explorations (pp. 71-100). New York, NY: Oxford University Press.

Wilson, D.B. (2005). Meta-analysis macros for SAS, SPSS, and Stata. Retrieved from http://mason.gmu.edu/ dwilsonb/ma.html

Winchie, D.B., \& Carment, D.W. (1989). Migration and motivation: The migrant's perspective. International Migration Review, 23(1), 96-104. 
Yano, Y. (2009). English as an international lingua franca: From societal to individual. World Englishes, 28(2), 246-255. doi:10.1111/j.1467-971X.2009.01587.x

Yoo, S.H., Matsumoto, D., \& LeRoux, J.A. (2006). The influence of emotion recognition and emotion regulation on intercultural adjustment. International Journal of Intercultural Relations, 30, 345-363.

Yoon, E., Hacker, J., Hewitt, A., Abrams, M., \& Cleary, S. (2012). Social connectedness, discrimination, and social status as mediators of acculturation/enculturation and wellbeing. Journal of Counseling Psychology, 59(1), 86-96. doi:10.1037/a0025366

Yoon, E., Lee, D.Y., Koo, Y.R., \& Yoo, S.K. (2009). A qualitative investigation of Korean immigrant women's lives. The Counseling Psychologist, 38(4), 523-553. doi:10.1177/0011000009346993

Yu, B. (2010). Learning Chinese abroad: The role of language attitudes and motivation in the adaptation of international students in China. Journal of Multilingual and Multicultural Development, 31(3), 301-321. doi:10.1080/01434631003735483

Yusoff, Y.M. (2010). Demographic differences among international undergraduate students at a Malaysian public university. Global Journal of Management and Business Research, 10(2), 36-41.

Zhang, J., \& Goodson, P. (2011). Acculturation and psychosocial adjustment of Chinese international students: Examining mediation and moderation effects. International Journal of Intercultural Relations, 35(3), 614-627. doi:10.1016/j.ijintrel.2010.11.004

Zhang, L. (2005). Malleability of cognitive style and its implications for management practice. Unpublished doctoral dissertation. Leeds University.

Zimmerman, B.J. (1994). Impact of self-regulatory influences on writing course attainment. American Educational Research Journal, 31(4), 845-862.

Zlobina, A., Basabe, N., Paez, D., \& Furnham, A. (2006). Sociocultural adjustment of immigrants: Universal and group-specific predictors. International Journal of Intercultural Relations, 30(2), 195-211. doi:10.1016/j.ijintrel.2005.07.005

Zung, W.K. (1972). The depression status inventory: An adjunct to the self-rating depression scale. Journal of Clinical Psychology, 28(4), 539-543. doi:10.1002/10974679(197210)28:4<539::AID-JCLP2270280427>3.0.CO;2-S

Zung, W.K., Richards, C.B., \& Short, M.J. (1965). Self-rating depression scale in an outpatient clinic. Archives of General Psychiatry, 13(6), 508-515.

Zuroff, D.C., Koestner, R., Moskowitz, D.S., McBride, C., Marshall, M., \& Bagby, M.R. (2007). Autonomous motivation for therapy: A new common factor in brief treatments 
for depression. Psychotherapy Research, 17(2), 137-147. doi:10.1080/10503300600919380 
Appendix A. Study Characteristics of the Meta-Analysis

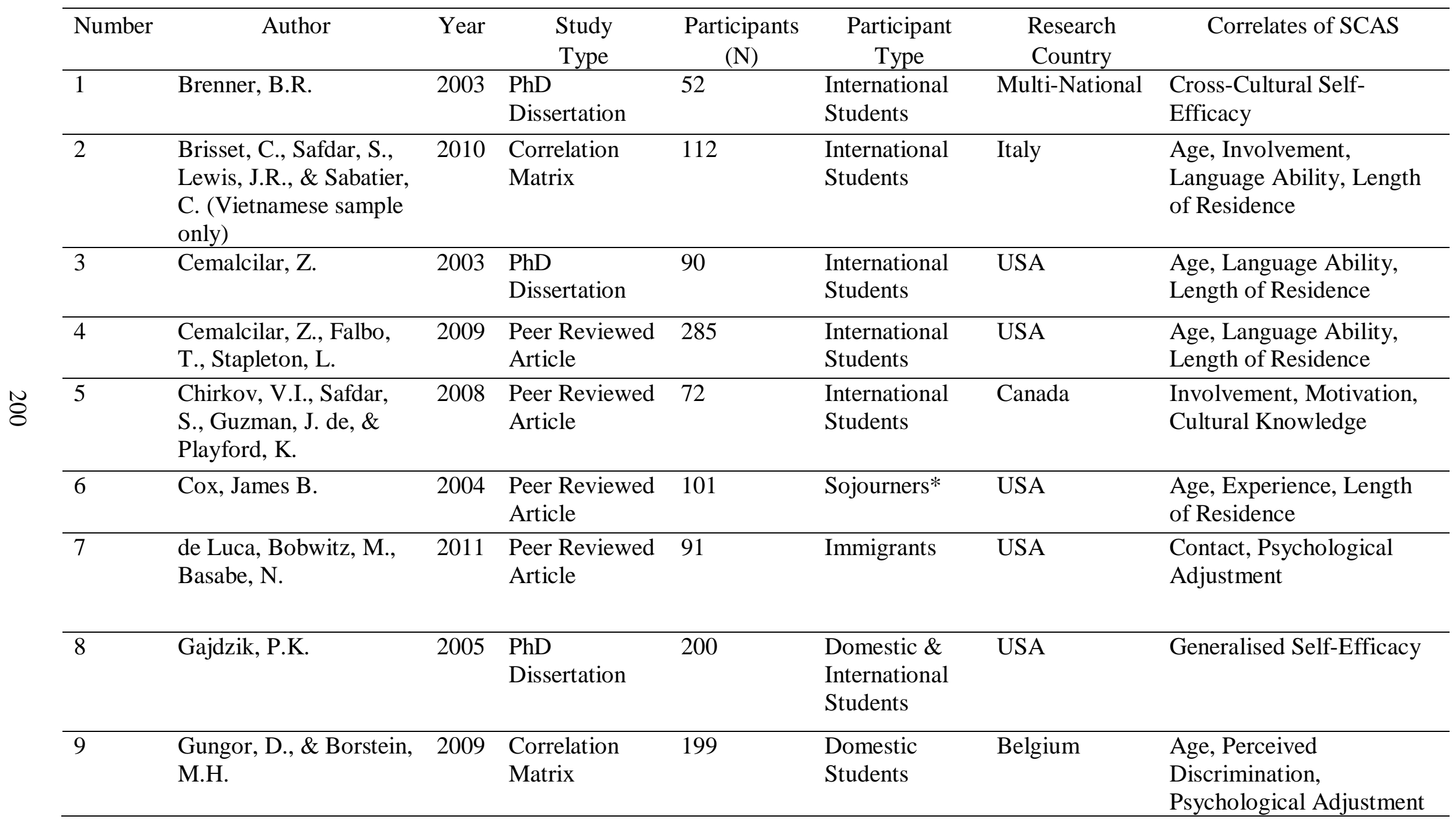




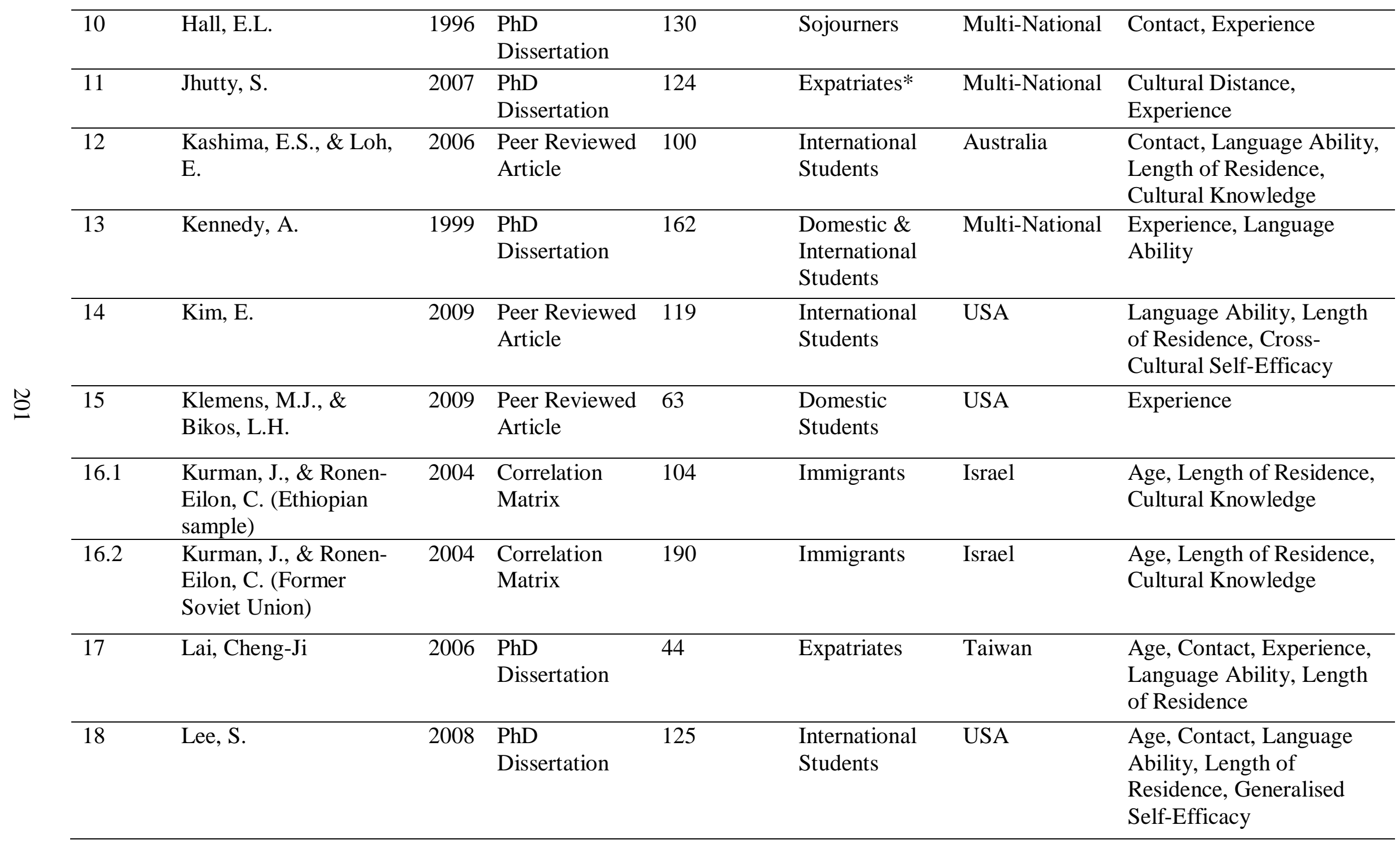




\begin{tabular}{|c|c|c|c|c|c|c|c|}
\hline 19 & Leong, C.-H. & 2007 & $\begin{array}{l}\text { Peer Reviewed } \\
\text { Article }\end{array}$ & 288 & $\begin{array}{l}\text { Domestic \& } \\
\text { International } \\
\text { Students }\end{array}$ & Multi-National & Psychological Adjustment \\
\hline 20 & Li, A., \& Gasser, M.B. & 2005 & $\begin{array}{l}\text { Peer Reviewed } \\
\text { Article }\end{array}$ & 117 & $\begin{array}{l}\text { International } \\
\text { Students }\end{array}$ & USA & $\begin{array}{l}\text { Contact, Cross-Cultural } \\
\text { Self-Efficacy }\end{array}$ \\
\hline 21 & Masgoret, A.M. & 2002 & $\begin{array}{l}\mathrm{PhD} \\
\text { Dissertation }\end{array}$ & 127 & $\begin{array}{l}\text { International } \\
\text { Students }\end{array}$ & Multi-National & $\begin{array}{l}\text { Contact, Cultural Distance, } \\
\text { Experience, Language } \\
\text { Ability, Motivation, } \\
\text { Length of Residence }\end{array}$ \\
\hline 23 & Moore, T.M. & 2009 & $\begin{array}{l}\mathrm{PhD} \\
\text { Dissertation }\end{array}$ & 120 & Combination & USA & $\begin{array}{l}\text { Age, Cultural Distance, } \\
\text { Language Ability }\end{array}$ \\
\hline 24 & $\begin{array}{l}\text { Oguri, M., \& } \\
\text { Gudykunst, W.B. }\end{array}$ & 2002 & $\begin{array}{l}\text { Peer Reviewed } \\
\text { Article }\end{array}$ & 175 & $\begin{array}{l}\text { International } \\
\text { Students }\end{array}$ & USA & Psychological Adjustment \\
\hline 27 & Shim, I.-S. & 2001 & $\begin{array}{l}\mathrm{PhD} \\
\text { Dissertation }\end{array}$ & 70 & Expatrates & Multi-National & $\begin{array}{l}\text { Age, Experience, } \\
\text { Language Ability }\end{array}$ \\
\hline 28 & Shupe, E. & 2007 & $\begin{array}{l}\text { Peer Reviewed } \\
\text { Article }\end{array}$ & 203 & $\begin{array}{l}\text { International } \\
\text { Students }\end{array}$ & USA & Cultural Distance \\
\hline 29 & $\begin{array}{l}\text { Spencer-Oatey, H., \& } \\
\text { Xiong, Z. }\end{array}$ & 2006 & $\begin{array}{l}\text { Non-Peer } \\
\text { Reviewed } \\
\text { Article }\end{array}$ & 126 & $\begin{array}{l}\text { International } \\
\text { Students }\end{array}$ & England & Psychological Adjustment \\
\hline 30.1 & Sugiura, T. (Study 1) & 2004 & $\begin{array}{l}\mathrm{PhD} \\
\text { Dissertation }\end{array}$ & 137 & $\begin{array}{l}\text { International } \\
\text { Students }\end{array}$ & Australia & $\begin{array}{l}\text { Contact, Experience, } \\
\text { Language Ability }\end{array}$ \\
\hline
\end{tabular}




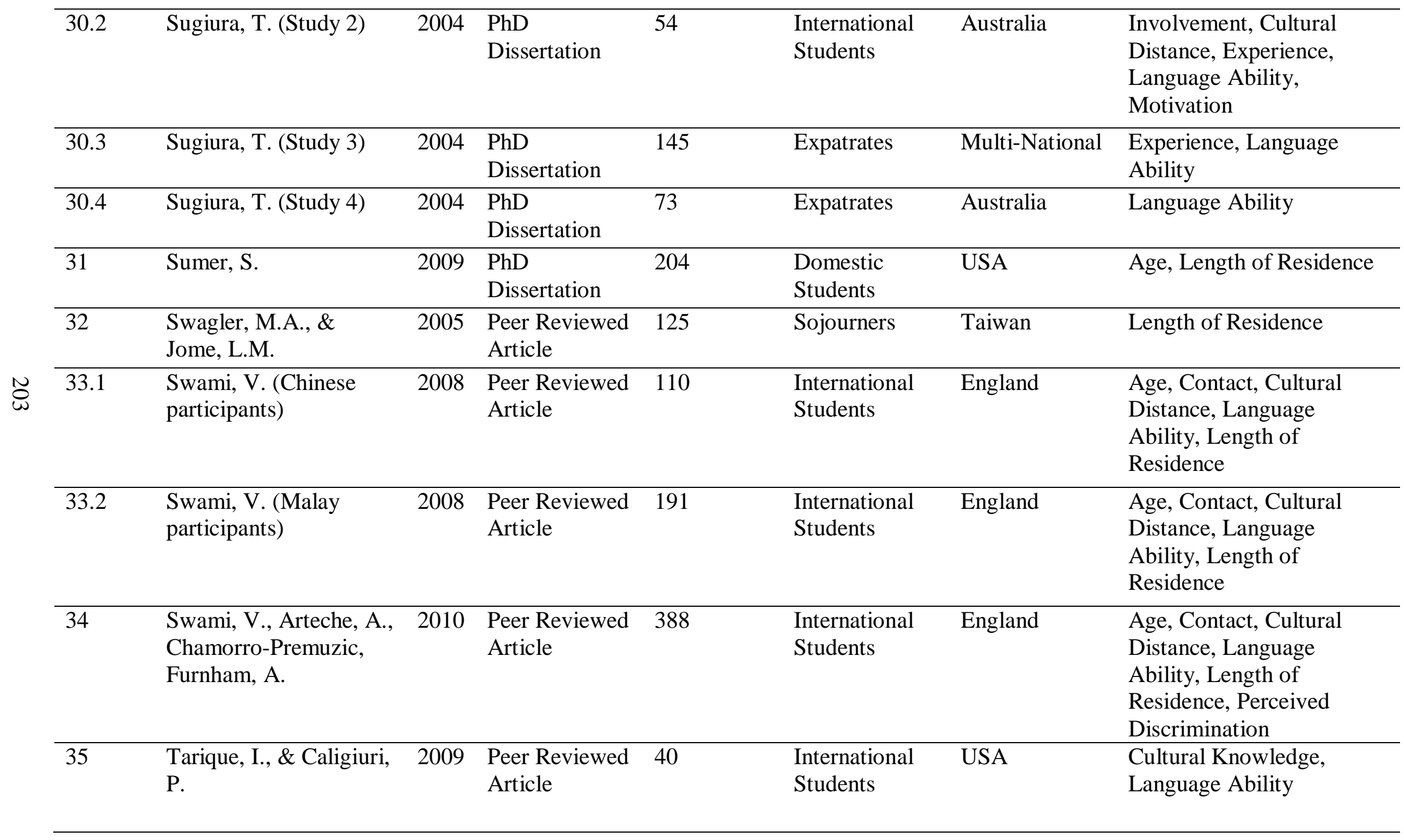




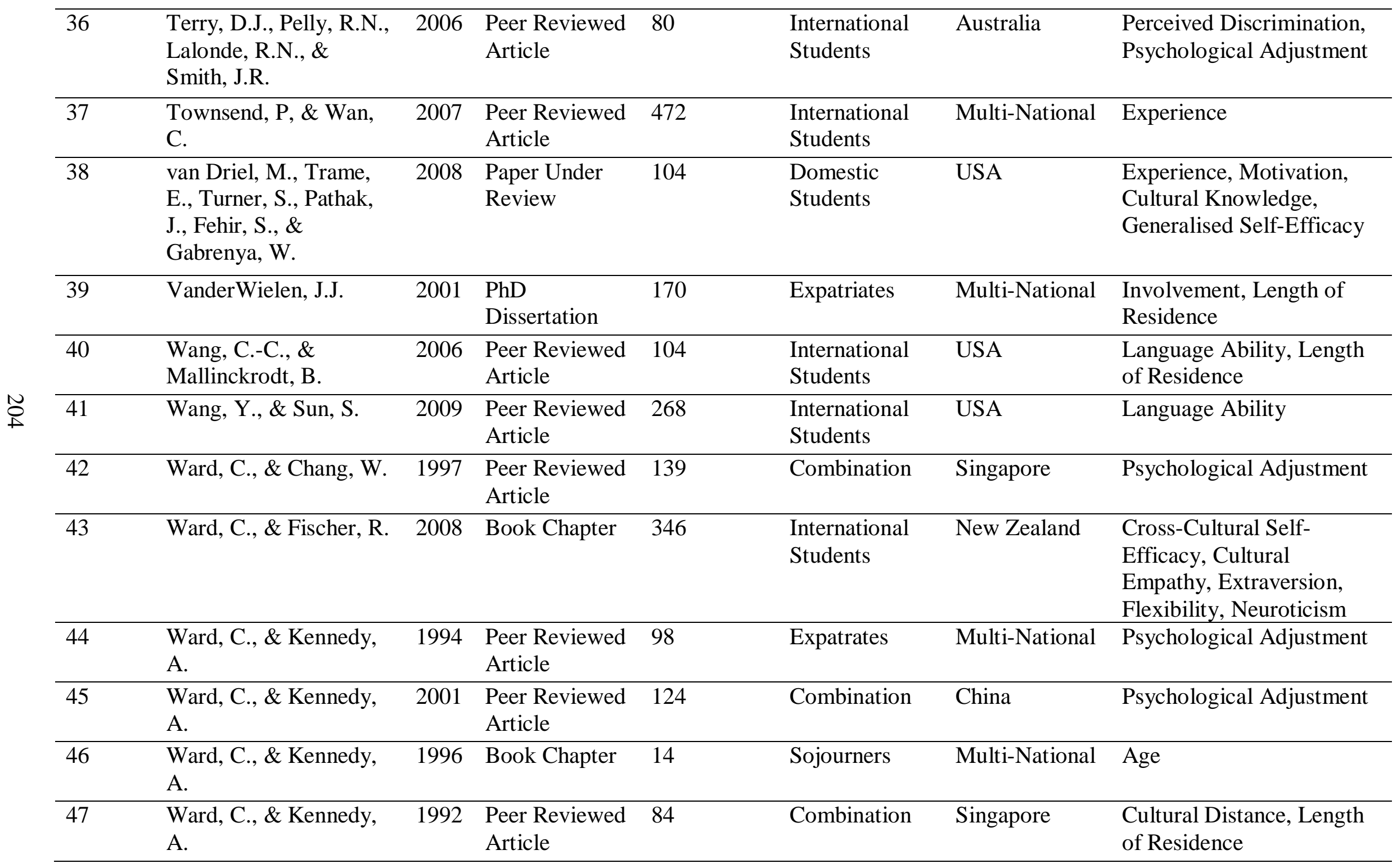




\begin{tabular}{|c|c|c|c|c|c|c|c|}
\hline 48.1 & $\begin{array}{l}\text { Ward, C., \& Kennedy, } \\
\text { A. (Study 1) }\end{array}$ & 1996 & Book Chapter & 301 & $\begin{array}{l}\text { Domestic \& } \\
\text { International } \\
\text { Students } \\
\end{array}$ & Multi-National & Psychological Adjustment \\
\hline 48.2 & $\begin{array}{l}\text { Ward, C., \& Kennedy, } \\
\text { A. (Study 2) }\end{array}$ & 1996 & Book Chapter & 319 & $\begin{array}{l}\text { Domestic \& } \\
\text { International } \\
\text { Students } \\
\end{array}$ & Multi-National & Psychological Adjustment \\
\hline 49.1 & $\begin{array}{l}\text { Ward, C., \& Kennedy, } \\
\text { A. (Study 1) }\end{array}$ & 1993 & $\begin{array}{l}\text { Peer Reviewed } \\
\text { Article }\end{array}$ & 145 & $\begin{array}{l}\text { International } \\
\text { Students }\end{array}$ & New Zealand & $\begin{array}{l}\text { Contact, Cultural Distance, } \\
\text { Experience, Length of } \\
\text { Residence }\end{array}$ \\
\hline 49.2 & $\begin{array}{l}\text { Ward, C., \& Kennedy, } \\
\text { A. (Study 2) }\end{array}$ & 1993 & $\begin{array}{l}\text { Peer Reviewed } \\
\text { Article }\end{array}$ & 156 & $\begin{array}{l}\text { International } \\
\text { Students }\end{array}$ & Singapore & $\begin{array}{l}\text { Contact, Cultural Distance, } \\
\text { Experience, Length of } \\
\text { Residence }\end{array}$ \\
\hline 50.1 & $\begin{array}{l}\text { Ward, C., \& Kennedy, } \\
\text { A. (Study 1) }\end{array}$ & 1993 & $\begin{array}{l}\text { Peer Reviewed } \\
\text { Article }\end{array}$ & 178 & $\begin{array}{l}\text { International } \\
\text { Students }\end{array}$ & Multi-National & $\begin{array}{l}\text { Contact, Cultural Distance, } \\
\text { Language Ability, Length } \\
\text { of Residence }\end{array}$ \\
\hline 50.2 & $\begin{array}{l}\text { Ward, C., \& Kennedy, } \\
\text { A. (Study 2) }\end{array}$ & 1993 & $\begin{array}{l}\text { Peer Reviewed } \\
\text { Article }\end{array}$ & 142 & $\begin{array}{l}\text { Domestic } \\
\text { Students }\end{array}$ & New Zealand & Psychological Adjustment \\
\hline 51.1 & $\begin{array}{l}\text { Ward, C., Berno, T., \& } \\
\text { Main, A. (Study 1) }\end{array}$ & 2002 & Book Chapter & 95 & $\begin{array}{l}\text { International } \\
\text { Students }\end{array}$ & New Zealand & $\begin{array}{l}\text { Cultural Distance, } \\
\text { Perceived Discrimination }\end{array}$ \\
\hline 51.2 & $\begin{array}{l}\text { Ward, C., Berno, T., \& } \\
\text { Main, A. (Study 2) }\end{array}$ & 2002 & Book Chapter & 47 & $\begin{array}{l}\text { International } \\
\text { Students }\end{array}$ & Multi-National & Psychological Adjustment \\
\hline 52.1 & $\begin{array}{l}\text { Ward, C., Fischer, R., } \\
\text { Lam, F.S., \& Hall, L. } \\
\text { (Study 2) }\end{array}$ & 2009 & $\begin{array}{l}\text { Correlation } \\
\text { Matrix }\end{array}$ & 118 & $\begin{array}{l}\text { International } \\
\text { Students }\end{array}$ & New Zealand & $\begin{array}{l}\text { Age, Language Ability, } \\
\text { Motivation, Length of } \\
\text { Residence }\end{array}$ \\
\hline 52.2 & $\begin{array}{l}\text { Ward, C., Fischer, R., } \\
\text { Lam, F.S., \& Hall, L. } \\
\text { (Study 3) }\end{array}$ & 2009 & $\begin{array}{l}\text { Correlation } \\
\text { Matrix }\end{array}$ & 102 & $\begin{array}{l}\text { International } \\
\text { Students }\end{array}$ & New Zealand & $\begin{array}{l}\text { Age, Language Ability, } \\
\text { Motivation, Length of } \\
\text { Residence }\end{array}$ \\
\hline 53.1 & $\begin{array}{l}\text { Ward, C., Leong, C.-H., } \\
\text { \& Low, M. (Australian } \\
\text { sample data set) }\end{array}$ & 2004 & $\begin{array}{l}\text { Correlation } \\
\text { Matrix }\end{array}$ & 222 & Combination & Singapore & $\begin{array}{l}\text { Contact, Involvement, } \\
\text { Experience, Length of } \\
\text { Residence }\end{array}$ \\
\hline
\end{tabular}




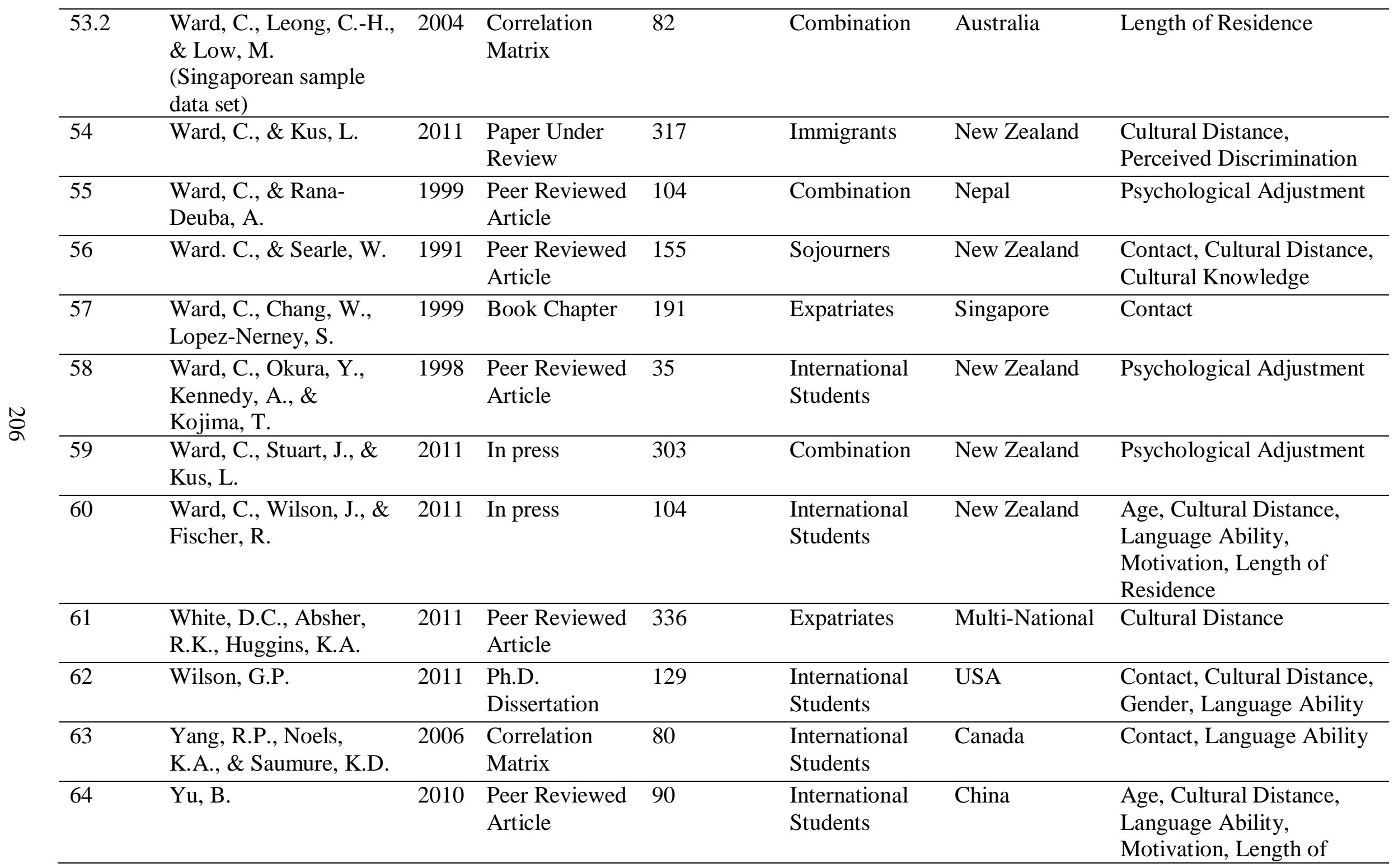


Residence

\begin{tabular}{|c|c|c|c|c|c|c|c|}
\hline 65 & Yu, B., \& Shen, H. & 2011 & $\begin{array}{l}\text { Peer Reviewed } \\
\text { Article }\end{array}$ & 198 & $\begin{array}{l}\text { International } \\
\text { Students }\end{array}$ & Australia & $\begin{array}{l}\text { Age, Gender, Integrative } \\
\text { Motivation, Language } \\
\text { Ability, Length of } \\
\text { Residence }\end{array}$ \\
\hline 66 & Yu, B.H. & 2005 & $\begin{array}{l}\mathrm{PhD} \\
\text { Dissertation }\end{array}$ & 257 & $\begin{array}{l}\text { International } \\
\text { Students }\end{array}$ & China & $\begin{array}{l}\text { Language Ability, } \\
\text { Motivation }\end{array}$ \\
\hline 67 & $\begin{array}{l}\text { Zhang, J., \& Goodson, } \\
\text { P. }\end{array}$ & 2010 & $\begin{array}{l}\text { Paper Under } \\
\text { Review }\end{array}$ & 508 & $\begin{array}{l}\text { International } \\
\text { Students }\end{array}$ & USA & Contact \\
\hline 68 & $\begin{array}{l}\text { Zlobina, A., Basabe, N., } \\
\text { Paez, D., \& Furnham, } \\
\text { A. }\end{array}$ & 2006 & $\begin{array}{l}\text { Peer Reviewed } \\
\text { Article }\end{array}$ & 518 & Immigrants & Spain & $\begin{array}{l}\text { Age, Contact, Cultural } \\
\text { Distance, Length of } \\
\text { Residence, Perceived } \\
\text { Discrimination }\end{array}$ \\
\hline 69 & $\begin{array}{l}\text { Zokaitluangi, \& Varte, } \\
\text { C.L. }\end{array}$ & 2005 & Book Chapter & 535 & $\begin{array}{l}\text { Domestic } \\
\text { Students }\end{array}$ & Italy & Psychological Adjustment \\
\hline
\end{tabular}

* Sojourner groups include missionaries, and volunteers

* Combination groups include mixtures of expats, immigrants, sojourners, spouses, students, and/or volunteers

* Expatriates include managers and employees 
Appendix B. Overview of Multi-National Studies

\begin{tabular}{|c|c|c|c|}
\hline $\begin{array}{l}\text { Study } \\
\text { Number }\end{array}$ & Author & Study Title & Research Countries \\
\hline 1 & Brenner, B.R. & $\begin{array}{l}\text { A study of self-awareness, self-efficacy, and } \\
\text { sojourner adjustment over time }\end{array}$ & $\begin{array}{l}\text { United States-based universities with exchange } \\
\text { students in } 12 \text { countries (e.g., France, Germany, } \\
\text { Hong Kong, Ireland, Russia) }\end{array}$ \\
\hline 10 & Hall, E.L. & $\begin{array}{l}\text { The relationship of object relations } \\
\text { development to cultural adjustment }\end{array}$ & $\begin{array}{l}\text { United States-based missionary organisation with } \\
\text { missionaries in } 41 \text { countries (e.g., Russia, Tanzania, } \\
\text { Kenya, France, Ukraine) }\end{array}$ \\
\hline 11 & Jhutty, S. & $\begin{array}{l}\text { Emotional intelligence and expatriate cross- } \\
\text { cultural adjustment }\end{array}$ & $\begin{array}{l}\text { Expatriates in } 41 \text { countries (e.g., United States, } \\
\text { England, Thailand, China, Germany) }\end{array}$ \\
\hline 13 & Kennedy, A. & $\begin{array}{l}\text { Singaporean sojourners: Meeting the demands } \\
\text { of cross-cultural transitions }\end{array}$ & $\begin{array}{l}\text { Singapore-based university with exchange students } \\
\text { in various countries (e.g., Australia, New Zealand, } \\
\text { China, the United States) }\end{array}$ \\
\hline 19 & Leong, C.-H. & $\begin{array}{l}\text { Predictive validity of the Multicultural } \\
\text { Personality Questionnaire: A longitudinal study } \\
\text { on the socio-psychological adaptation of Asian } \\
\text { undergraduates who took part in a study-abroad } \\
\text { program }\end{array}$ & Singapore, Not Listed \\
\hline 21 & Masgoret, A.M. & $\begin{array}{l}\text { Investigating cross-cultural adjustment, and the } \\
\text { influence of foreign language instructors on } \\
\text { second language achievement }\end{array}$ & England, Spain \\
\hline 27 & Shim, I.-S. & $\begin{array}{l}\text { Factors that facilitate or limit expatriates' } \\
\text { adapting and adjusting to another country }\end{array}$ & $\begin{array}{l}\text { United States-based organisation with expatriates in } \\
19 \text { countries (e.g., Argentina, Belgium, Dubai, } \\
\text { China) }\end{array}$ \\
\hline
\end{tabular}



30.3
Sugiura, T. (Study 3)
Adjustment patterns of international students
Australia, Japan and expatriate managers.

37 Townsend, P, \& Wan, C. The impact of multicultural experience in the development of socio-cultural adaptation for

Australia, Malaysia international business students.

$39 \quad$ VanderWielen, J.J.

Cognitive appraisal, coping, and the psychological and sociocultural adjustment of

Expatriates in 37 countries (e.g., England, United expatriates

\begin{tabular}{|c|c|c|c|}
\hline 44 & Ward, C., \& Kennedy, A. & $\begin{array}{l}\text { Acculturation strategies, psychological } \\
\text { adjustment, and sociocultural competence } \\
\text { during cross-cultural transitions }\end{array}$ & $\begin{array}{l}\text { New Zealand-based international organisation with } \\
\text { employees in at least } 27 \text { countries (e.g., China, } \\
\text { Indonesia, Japan, Mexico, United States) }\end{array}$ \\
\hline 46 & Ward, C., \& Kennedy, A. & $\begin{array}{l}\text { Before and after cross-cultural transition: A } \\
\text { study of New Zealand volunteers on field } \\
\text { assignments }\end{array}$ & $\begin{array}{l}\text { New Zealand-based volunteer organisation with } \\
\text { members in } 8 \text { countries (e.g., Bhutan, Cook Islands, } \\
\text { Nambia, Tanzania) }\end{array}$ \\
\hline 48.1 & $\begin{array}{l}\text { Ward, C., \& Kennedy, A. } \\
\text { (Study 1) }\end{array}$ & $\begin{array}{l}\text { Crossing cultures: The relationship between } \\
\text { psychological and sociocultural dimensions of } \\
\text { cross-cultural adjustment }\end{array}$ & New Zealand, Singapore \\
\hline 48.2 & $\begin{array}{l}\text { Ward, C., \& Kennedy, A. } \\
\text { (Study 2) }\end{array}$ & $\begin{array}{l}\text { Crossing cultures: The relationship between } \\
\text { psychological and sociocultural dimensions of } \\
\text { cross-cultural adjustment }\end{array}$ & $\begin{array}{l}\text { Study abroad students in } 23 \text { countries (e.g., } \\
\text { Argentina, Germany, Hong Kong, Malaysia, United } \\
\text { States) }\end{array}$ \\
\hline
\end{tabular}


51.2 Ward, C., Berno, T., \& Main, A. (Study 2)

61 White, D.C., Absher, R.K., Huggins, K.A.
Can the Cross-cultural Adaptability Inventory predict sojourner adjustment?

The effects of hardiness and cultural distance on sociocultural adaptation in an expatriate sales manager population
Australia, Singapore

Expatriates in 63 countries (e.g., Argentina, Brazil, China, Egypt, Jamaica, Japan, Sri Lanka, Ukraine, Zimbabwe) 
Appendix C. Number of Participants by Host Country

\begin{tabular}{|c|c|c|}
\hline Host Country & $\mathrm{N}$ & $\begin{array}{c}\text { Valid } \\
\%\end{array}$ \\
\hline New Zealand & 193 & 67.01 \\
\hline USA & 9 & 3.13 \\
\hline Italy & 6 & 2.08 \\
\hline Australia & 6 & 2.08 \\
\hline Japan & 5 & 1.74 \\
\hline Britain (UK) & 5 & 1.74 \\
\hline Denmark & 4 & 1.39 \\
\hline United Arab Emirates & 4 & 1.39 \\
\hline Switzerland & 3 & 1.04 \\
\hline Singapore & 3 & 1.04 \\
\hline Germany & 3 & 1.04 \\
\hline Holland (Netherlands) & 3 & 1.04 \\
\hline Taiwan & 2 & .69 \\
\hline Sweden & 2 & .69 \\
\hline Russia & 2 & .69 \\
\hline India & 2 & .69 \\
\hline France & 2 & .69 \\
\hline Belgium & 2 & .69 \\
\hline Austria & 2 & .69 \\
\hline Saudi Arabia & 1 & .35 \\
\hline Morocco & 1 & .35 \\
\hline Egypt & 1 & .35 \\
\hline Dominican Republic & 1 & .35 \\
\hline Spain & 1 & .35 \\
\hline Malaysia & 1 & .35 \\
\hline South Korea & 1 & .35 \\
\hline Hungary & 1 & .35 \\
\hline $\begin{array}{l}\text { China (also Hong } \\
\text { Kong) }\end{array}$ & 1 & .35 \\
\hline Chile & 1 & .35 \\
\hline Argentina & 1 & .35 \\
\hline Missing & 28 & \\
\hline Total & 316 & 100.00 \\
\hline
\end{tabular}




\section{VICTORIA UNIVERSITY OF WELLINGTON \\ Te Whare Wananga o te Upoko o te Ika a Maui}

\section{Researchers:}

Jessie Wilson (PhD Candidate) and Professor Colleen Ward (Research Supervisor), School of Psychology Email: jessie.wilson@vuw.ac.nz or 04 (463 5311) and colleen.ward@vuw.ac.nz.

\section{What is the purpose of this research?}

This research concerns how individuals adapt to living in new cultural environments.

\section{Who is conducting the research?}

- I am conducting this research as part of my PhD through the Centre for Applied Cross-cultural Research and School of Psychology at Victoria University of Wellington under the supervision of my advisor, Colleen Ward.

\section{What is involved if you agree to participate in the research?}

- Your participation is VOLUNTARY, and participating in this research implies your consent.

- If you agree to participate in this study, you will be given a link to an anonymous online survey to complete. In the survey, you will be asked to answer questions about behaviours such as "attending or participating in community activities" and "building and maintaining relationships". The survey will take no more than 60 minutes for you to complete.

- During the survey, you are free to withdraw at any point before the survey has been completed.

- You must be an international student or short-term migrant who is not currently living in your home country, and who has only lived in your new country of residence for less than two years to participate in the survey.

- If you are under 16 years of age, you cannot participate in the survey.

\section{Privacy and Confidentiality}

- I cannot personally identify you or match your survey responses with a name or address. This survey is completely anonymous, and you are not asked to put your name anywhere on the survey.

- If you complete the entire survey, you can enter a lucky draw for an iPod or a gift voucher. After the survey, there will be a separate contact information sheet regarding the lucky draw for you to complete if you wish that is not connected to your survey responses.

\section{What happens to the information that you provide?}

- I will keep your completed survey for at least five years after publication of my results. The surveys will be stored in a secure location in the School of Psychology, Victoria University of Wellington.

- In accordance with the requirements of some scientific journals and organizations, the coded survey responses may be shared with other competent researchers.

- The coded responses may be used in other, related studies.

- A copy of the coded survey responses will remain in my custody (Jessie Wilson).

- Preliminary results from the study will posted on the Centre for Applied Cross-Cultural Research website (http://www.vuw.ac.nz/cacr/) in October 2010.

- The information you provide may be submitted for publication in a scientific journal or presented at scientific conferences.

Thank you for considering participation in this research. Jessie Wilson and Colleen Ward 
People experience change when moving to a new culture. Such change often involves learning new skills and behaviours. The following items ask about your experiences in New Zealand. Please rate how competent you are at each of the following behaviours $(1=$ not at all competent; $5=$ extremely competent; 0 = not applicable).

\begin{tabular}{|c|c|c|c|c|c|}
\hline & $\begin{array}{c}\text { Not at all } \\
\text { competent }\end{array}$ & & & & $\begin{array}{l}\text { Extremely } \\
\text { competent }\end{array}$ \\
\hline 1. Buying food I like. & (1) & (2) & (3) & (4) & (5) \\
\hline 2. Dressing appropriately for the climate. & (1) & (2) & (3) & (4) & (5) \\
\hline 3. Maintaining my hobbies and interests. & (1) & (2) & (3) & (4) & (5) \\
\hline 4. Adapting to my accommodation. & (1) & (2) & (3) & (4) & (5) \\
\hline 5. Acquiring things I need or want. & (1) & (2) & (3) & (4) & (5) \\
\hline 6. Adapting to the pace of life. & (1) & (2) & (3) & (4) & (5) \\
\hline 7. Maintaining my religious practices. & (1) & (2) & (3) & (4) & (5) \\
\hline 8. Finding my way around. & (1) & (2) & (3) & (4) & (5) \\
\hline $\begin{array}{l}\text { 9. Adapting to the noise level in my } \\
\text { neighborhood. }\end{array}$ & (1) & (2) & (3) & (4) & (5) \\
\hline $\begin{array}{l}\text { 10. Dressing appropriately in various social } \\
\text { settings. }\end{array}$ & (1) & (2) & (3) & (4) & (5) \\
\hline 11. Adapting to the population density. & (1) & (2) & (3) & (4) & (5) \\
\hline 12. Using the transportation system. & (1) & (2) & (3) & (4) & (5) \\
\hline $\begin{array}{l}\text { 13. Seeking out and receiving appropriate } \\
\text { medical care. }\end{array}$ & (1) & (2) & (3) & (4) & (5) \\
\hline 14. Exercising my political or civic rights. & (1) & (2) & (3) & (4) & (5) \\
\hline $\begin{array}{l}\text { 15. Maintaining awareness of community } \\
\text { activities (e.g., arts, festivals, sports). }\end{array}$ & (1) & (2) & (3) & (4) & (5) \\
\hline 16. Engaging in political or civic activities. & (1) & (2) & (3) & (4) & (5) \\
\hline $\begin{array}{l}\text { 17. Attending or participating in community } \\
\text { activities. }\end{array}$ & (1) & (2) & (3) & (4) & (5) \\
\hline 18. Being aware of local news. & (1) & (2) & (3) & (4) & (5) \\
\hline 19. Dealing with the bureaucracy. & (1) & (2) & (3) & (4) & (5) \\
\hline
\end{tabular}


20. Obtaining community services I require.

(3)

(4)

21. Seeking out and receiving support from

(1)

(2)

(3)

(4)

(5) community organizations.

22. Reading and writing English.

(1)

(2)

(3) (4)

(5)

23. Changing my verbal behaviour (e.g., accent, tone) in a culturally appropriate manner.

(1)

(2)

(3)

(4)

(5)

24. Making myself understood.

(1)

(2)

(3) (4)

(5)

25. Understanding jokes and humour.

(1)

(2)

(3)

(4)

(5)

26. Building and maintaining relationships.

(1)

(2)

(3) (4)

(5)

27. Using pause and silence differently in a culturally appropriate manner.

(1)

(2)

(3)

(4)

(5)

28. Interacting with culturally diverse groups of people.

29. Solving new or unfamiliar problems.

(1)

(2)

(3)

(4)

(5)

30. Altering my facial expressions in a culturally appropriate manner.

(1) (3) (4)

(4) (5)

1. Accurately interpreting and responding to

other people's gestures and facial

(1)

(2)

(3)

(4)

(5)

expressions.

32. Asking for assistance when appropriate.

(1)

(2)

(3) (4)

(5)

33. Accurately interpreting other people's emotions.

(1)

(2)

(3)

(4)

(5)

34. Expressing my emotions to others in a culturally appropriate manner.

35. Understanding and speaking English.

(1)

(2)

(3)

(4)

(5)

(1)

(2)

(3)

(4)

(5)

36. Speaking about myself.

(1)

(2)

(3)

(4)

(5)

37. Altering my facial expressions in a culturally appropriate manner.

(1)

(2)

(3)

(4)

(5)

38. Performing routines like greetings or departures to suit different situations.

39. Interacting with members of the opposite sex.

(2)

(3)

(4)

(5)

(2)

(3)

(4)

(5)

40. Varying the rate of my speaking in a culturally appropriate manner.

(1)

(2)

(3)

(4)

(5) 
41. Interacting at social events.

(1)

(2)

(3)

(4)

(5)

42. Changing my behaviour to suit social norms, rules, attitudes, beliefs, and customs.

(1)

(2)

(3) (4)

(5)

43. Responding appropriately to corrective feedback.

(1)

(2)

(3)

(4)

(5)

44. Dealing with cultural misunderstandings.

(1)

(2)

(3) (4)

(5)

45. Listening to other people's point of view.

(1)

(2)

(3)

(4)

(5)

46. Completing academic assignments on time.

(1)

(2)

(3)

(4)

(5)

47. Performing my academic work well.

(1)

(2)

(3) (4)

(5)

48. Managing my academic responsibilities and work load.

(1)

(2)

(3)

(4)

(5)

49. Working effectively with other students and academic staff.

(1)

(2)

(3)

(4)

(5)

50. Passing academic exams.

(1)

(2)

(3)

(4)

(5)

51. Understanding the academic responsibilities expected of me.

(1)

(2)

(3)

(4)

(5)

52. Expressing my ideas in class in a culturally appropriate manner.

(1)

(2)

(3)

(4)

(5)

53. Gaining feedback from other students and academic staff to help improve my performance.

54. Attending lectures, tutorials, and other academic-related events on a regular basis.

(1)

(2)

(3)

(4)

(5)

(1)

(2)

(3)

(4)

(5) 
Please indicate how unadjusted or adjusted you are to the following items ( 1 = not adjusted at all; 7 = completely adjusted). Also, please note that a "host national" is someone who was born in your host country. Your host country is the country in which you currently live.

\begin{tabular}{|c|c|c|c|c|c|c|c|}
\hline & $\begin{array}{c}\text { Not } \\
\text { adjusted } \\
\text { all }\end{array}$ & & & & & & $\begin{array}{c}\text { Completel } \\
\text { y adjusted }\end{array}$ \\
\hline 1. Living conditions in general & (1) & (2) & (3) & (4) & (5) & (6) & (7) \\
\hline 2. Housing conditions & (1) & (2) & (3) & (4) & (5) & (6) & (7) \\
\hline 3. Food & (1) & (2) & (3) & (4) & (5) & (6) & (7) \\
\hline 4. Shopping & (1) & (2) & (3) & (4) & (5) & (6) & (7) \\
\hline 5. Cost of living & (1) & (2) & (3) & (4) & (5) & (6) & (7) \\
\hline $\begin{array}{l}\text { 6. Entertainment/recreation facilities and } \\
\text { opportunities }\end{array}$ & (1) & (2) & (3) & (4) & (5) & (6) & (7) \\
\hline 7. Health care facilities & (1) & (2) & (3) & (4) & (5) & (6) & (7) \\
\hline 8. Socializing with host nationals & (1) & (2) & (3) & (4) & (5) & (6) & (7) \\
\hline $\begin{array}{l}\text { 9. Interacting with host nationals on a day-to-day } \\
\text { basis }\end{array}$ & (1) & (2) & (3) & (4) & (5) & (6) & (7) \\
\hline $\begin{array}{l}\text { 10. Interacting with host nationals outside of } \\
\text { university }\end{array}$ & (1) & (2) & (3) & (4) & (5) & (6) & (7) \\
\hline 11. Speaking with host nationals & (1) & (2) & (3) & (4) & (5) & (6) & (7) \\
\hline 12. Specific academic responsibilities & (1) & (2) & (3) & (4) & (5) & (6) & (7) \\
\hline $\begin{array}{l}\text { 13. Academic performance standards and } \\
\text { expectations }\end{array}$ & (1) & (2) & (3) & (4) & (5) & (6) & (7) \\
\hline
\end{tabular}


Please indicate how strongly you agree with the following statements $(1=$ strongly disagree; $5=$ strongly agree).

\section{Strongly}

Disagree
Strongly Agree
1. In most ways my life is close to my ideal.

2. The conditions of my life are excellent.

3. I am satisfied with my life.

4. So far I have got the important things I want in life.

5. If I could live my life over, I would change almost nothing.
(1)

(1)

(1)

(1)

(1)

(2)

(3)

(4)

(2) (3) (4)

(4)
(5)

(2) (3) (4) (5)

(5)

(2) (3) (4) (5)

(5) 
Please indicate how often you perform the following behaviours $(1=$ never; $7=$ daily or almost daily). Again, please note that a "host national" is someone who was born in your host country.

\begin{tabular}{|c|c|c|c|c|c|c|c|}
\hline & Never & $\begin{array}{l}\text { Two or } \\
\text { three } \\
\text { times a } \\
\text { year }\end{array}$ & $\begin{array}{l}\text { Once a } \\
\text { month }\end{array}$ & $\begin{array}{l}\text { Two or } \\
\text { three } \\
\text { times a } \\
\text { month }\end{array}$ & $\begin{array}{l}\text { Once a } \\
\text { week }\end{array}$ & $\begin{array}{l}\text { Two or } \\
\text { three } \\
\text { times a } \\
\text { week }\end{array}$ & $\begin{array}{l}\text { Daily or } \\
\text { almost } \\
\text { daily }\end{array}$ \\
\hline $\begin{array}{l}\text { 1. How often do you eat New } \\
\text { Zealand dishes? }\end{array}$ & (1) & (2) & (3) & (4) & (5) & (6) & (7) \\
\hline $\begin{array}{l}\text { 2. How often do you eat } \\
\text { dishes of your own country? }\end{array}$ & (1) & (2) & (3) & (4) & (5) & (6) & (7) \\
\hline $\begin{array}{l}\text { 3. How often do you speak } \\
\text { the host language? }\end{array}$ & (1) & (2) & (3) & (4) & (5) & (6) & (7) \\
\hline $\begin{array}{l}\text { 4. How often do you speak } \\
\text { your own language? }\end{array}$ & (1) & (2) & (3) & (4) & (5) & (6) & (7) \\
\hline $\begin{array}{l}\text { 5. How often do you socialize } \\
\text { with New Zealander friends? }\end{array}$ & (1) & (2) & (3) & (4) & (5) & (6) & (7) \\
\hline $\begin{array}{l}\text { 6. How often do you socialize } \\
\text { with friends from your own } \\
\text { country? }\end{array}$ & (1) & (2) & (3) & (4) & (5) & (6) & (7) \\
\hline $\begin{array}{l}\text { 7. How often do you ask for } \\
\text { help/advice of New } \\
\text { Zealander students? }\end{array}$ & (1) & (2) & (3) & (4) & (5) & (6) & (7) \\
\hline $\begin{array}{l}\text { 8. How often do you ask for } \\
\text { help/advice of students from } \\
\text { your own country? }\end{array}$ & (1) & (2) & (3) & (4) & (5) & (6) & (7) \\
\hline $\begin{array}{l}\text { 9. How often do you gather } \\
\text { information about what is } \\
\text { happening in New Zealand? }\end{array}$ & (1) & (2) & (3) & (4) & (5) & (6) & (7) \\
\hline $\begin{array}{l}\text { 10. How often do you gather } \\
\text { information about what is } \\
\text { happening in your own } \\
\text { country? }\end{array}$ & (1) & (2) & (3) & (4) & (5) & (6) & (7) \\
\hline $\begin{array}{l}\text { 11. How often do you listen } \\
\text { to New Zealander music? }\end{array}$ & (1) & (2) & (3) & (4) & (5) & (6) & (7) \\
\hline $\begin{array}{l}\text { 12. How often do you listen } \\
\text { to music of your own } \\
\text { country? }\end{array}$ & (1) & (2) & (3) & (4) & (5) & (6) & (7) \\
\hline $\begin{array}{l}\text { 13. How often do you } \\
\text { participate in New Zealand } \\
\text { parties? }\end{array}$ & (1) & (2) & (3) & (4) & (5) & (6) & (7) \\
\hline $\begin{array}{l}\text { 14. How often do you } \\
\text { participate in parties of } \\
\text { your own country? }\end{array}$ & (1) & (2) & (3) & (4) & (5) & (6) & (7) \\
\hline $\begin{array}{l}\text { 15. How often do you } \\
\text { participate in New Zealand } \\
\text { public celebrations? }\end{array}$ & (1) & (2) & (3) & (4) & (5) & (6) & (7) \\
\hline $\begin{array}{l}\text { 16. How often do you } \\
\text { participate in public } \\
\text { celebrations of your own } \\
\text { country? }\end{array}$ & (1) & (2) & (3) & (4) & (5) & (6) & (7) \\
\hline
\end{tabular}


Read each statement and select the response that best describes your capabilities. Select the answer that BEST describes you AS YOU REALLY ARE (1 = strongly disagree; 7 = strongly agree).

\begin{tabular}{|c|c|c|c|c|c|c|c|}
\hline & $\begin{array}{l}\text { Strongly } \\
\text { disagree }\end{array}$ & & & & & & $\begin{array}{c}\text { Strongly } \\
\text { agree }\end{array}$ \\
\hline $\begin{array}{l}\text { 1. I change my verbal behaviour } \\
\text { (e.g., accent, tone) when a cross- } \\
\text { cultural interaction requires it. }\end{array}$ & (1) & (2) & (3) & (4) & (5) & (6) & (7) \\
\hline $\begin{array}{l}\text { 2. I use pause and silence } \\
\text { differently to suit different cross- } \\
\text { cultural situations. }\end{array}$ & (1) & (2) & (3) & (4) & (5) & (6) & (7) \\
\hline $\begin{array}{l}\text { 3. I vary the rate of my speaking } \\
\text { when a cross-cultural situation } \\
\text { requires it. }\end{array}$ & (1) & (2) & (3) & (4) & (5) & (6) & (7) \\
\hline $\begin{array}{l}\text { 4. I change my nonverbal } \\
\text { behaviour when a cross-cultural } \\
\text { situation requires it. }\end{array}$ & (1) & (2) & (3) & (4) & (5) & (6) & (7) \\
\hline $\begin{array}{l}\text { 5. I alter my facial expressions } \\
\text { when a cross-cultural interaction } \\
\text { requires it. }\end{array}$ & (1) & (2) & (3) & (4) & (5) & (6) & (7) \\
\hline
\end{tabular}


Please indicate how frequently you experience the following $(1=$ a little of the time; $4=$ most of the time).

\begin{tabular}{|c|c|c|c|c|}
\hline & $\begin{array}{l}\text { A little of the } \\
\text { time }\end{array}$ & $\begin{array}{l}\text { Some of the } \\
\text { time }\end{array}$ & $\begin{array}{l}\text { A good part } \\
\text { of the time }\end{array}$ & $\begin{array}{l}\text { Most of the } \\
\text { time }\end{array}$ \\
\hline 1. I feel sad. & (1) & (2) & (3) & (4) \\
\hline 2. Morning is when I feel best. & (1) & (2) & (3) & (4) \\
\hline 3. I have crying spells. & (1) & (2) & (3) & (4) \\
\hline 4. I have trouble sleeping at night. & (1) & (2) & (3) & (4) \\
\hline 5. I eat as much as I used to. & (1) & (2) & (3) & (4) \\
\hline 6. I notice that my weight has changed. & (1) & (2) & (3) & (4) \\
\hline 7. I have trouble with constipation. & (1) & (2) & (3) & (4) \\
\hline 8. My heart beats faster than usual. & (1) & (2) & (3) & (4) \\
\hline 9. I get tired for no reason. & (1) & (2) & (3) & (4) \\
\hline 10. My mind is as clear as it used to be. & (1) & (2) & (3) & (4) \\
\hline 11. I find it easy to do things I used to do. & (1) & (2) & (3) & (4) \\
\hline 12. I am restless and cannot keep still. & (1) & (2) & (3) & (4) \\
\hline 13. I am hopeful about the future. & (1) & (2) & (3) & (4) \\
\hline 14. I feel more irritable than I used to be. & (1) & (2) & (3) & (4) \\
\hline 15. I find it easy to make decisions. & (1) & (2) & (3) & (4) \\
\hline 16. I feel that I am useful and needed. & (1) & (2) & (3) & (4) \\
\hline 17. My life is meaningful. & (1) & (2) & (3) & (4) \\
\hline $\begin{array}{l}\text { 18. I feel that others would be better off if I } \\
\text { were dead. }\end{array}$ & (1) & (2) & (3) & (4) \\
\hline 19. I still enjoy the things I used to. & (1) & (2) & (3) & (4) \\
\hline
\end{tabular}


Please indicate how much difficulty you are experiencing in New Zealand in each of the areas below ( 1 = no difficulty; 5 = extreme difficulty).

\begin{tabular}{|c|c|c|c|c|c|}
\hline & $\begin{array}{c}\text { No } \\
\text { Difficult } \\
y\end{array}$ & $\begin{array}{l}\text { Slight } \\
\text { Difficult } \\
\quad \mathbf{y}\end{array}$ & $\begin{array}{c}\text { Moderat } \\
\mathbf{e} \\
\text { Difficult } \\
\mathbf{y}\end{array}$ & $\begin{array}{c}\text { Great } \\
\text { Difficult } \\
\quad \mathbf{y}\end{array}$ & $\begin{array}{l}\text { Extreme } \\
\text { Difficulty }\end{array}$ \\
\hline 1. Making friends & (1) & (2) & (3) & (4) & (5) \\
\hline 2. Following rules and regulations & (1) & (2) & (3) & (4) & (5) \\
\hline 3. Dealing with people of authority & (1) & (2) & (3) & (4) & (5) \\
\hline 4. Getting used to the local food & (1) & (2) & (3) & (4) & (5) \\
\hline 5. Using the transport system (eg., buses) & (1) & (2) & (3) & (4) & (5) \\
\hline 6. Dealing with bureaucracy & (1) & (2) & (3) & (4) & (5) \\
\hline 7. Making yourself understood & (1) & (2) & (3) & (4) & (5) \\
\hline 8. Going shopping & (1) & (2) & (3) & (4) & (5) \\
\hline 9. Dealing with someone who is unpleasant & (1) & (2) & (3) & (4) & (5) \\
\hline 10. Understanding jokes and humour & (1) & (2) & (3) & (4) & (5) \\
\hline 11. Going to parties and other social events & (1) & (2) & (3) & (4) & (5) \\
\hline 12. Talking about yourself and your feelings & (1) & (2) & (3) & (4) & (5) \\
\hline 13. Dealing with unsatisfactory service & (1) & (2) & (3) & (4) & (5) \\
\hline 14. Worshipping in your usual way & (1) & (2) & (3) & (4) & (5) \\
\hline 15. Relating to members of the opposite sex & (1) & (2) & (3) & (4) & (5) \\
\hline 16. Understanding ethnic or cultural differences & (1) & (2) & (3) & (4) & (5) \\
\hline 17. Finding your way around & (1) & (2) & (3) & (4) & (5) \\
\hline 18. Dealing with the climate & (1) & (2) & (3) & (4) & (5) \\
\hline 19. Getting used to the pace of life & (1) & (2) & (3) & (4) & (5) \\
\hline $\begin{array}{l}\text { 20. Adapting to the local accommodation (i.e., your living } \\
\text { situation) }\end{array}$ & (1) & (2) & (3) & (4) & (5) \\
\hline 21. Communicating with people of a different ethnic group & (1) & (2) & (3) & (4) & (5) \\
\hline 22. Understanding the local accent/language & (1) & (2) & (3) & (4) & (5) \\
\hline 23. Adapting to local etiquette or customs & (1) & (2) & (3) & (4) & (5) \\
\hline
\end{tabular}


Please read each question, and then select the option that best represents how you feel $(1=$ not at all like me; 5 = exactly like me).

\begin{tabular}{|c|c|c|c|c|c|}
\hline & $\begin{array}{l}\text { Not At } \\
\text { All Like } \\
\text { Me }\end{array}$ & $\begin{array}{l}\text { A Little } \\
\text { Like Me }\end{array}$ & $\begin{array}{c}\text { Like } \\
\text { Me }\end{array}$ & $\begin{array}{l}\text { Very } \\
\text { Much } \\
\text { Like Me }\end{array}$ & $\begin{array}{l}\text { Exactly } \\
\text { Like Me }\end{array}$ \\
\hline $\begin{array}{l}\text { 1. I am interested in knowing what motivates a } \\
\text { person's actions. }\end{array}$ & (1) & (2) & (3) & (4) & (5) \\
\hline 2. I can control my emotions when I need to. & (1) & (2) & (3) & (4) & (5) \\
\hline 3. I love to socialize. & (1) & (2) & (3) & (4) & (5) \\
\hline $\begin{array}{l}\text { 4. There are certain situations in which I find } \\
\text { myself worrying about whether I am doing or } \\
\text { saying the right things. }\end{array}$ & (1) & (2) & (3) & (4) & (5) \\
\hline 5. I am comfortable around all types of people. & (1) & (2) & (3) & (4) & (5) \\
\hline $\begin{array}{l}\text { 6. I can easily tell what a person's character is } \\
\text { by watching his or her interactions with } \\
\text { others. }\end{array}$ & (1) & (2) & (3) & (4) & (5) \\
\hline $\begin{array}{l}\text { 7. I often worry that people will misinterpret } \\
\text { something I have said to them. }\end{array}$ & (1) & (2) & (3) & (4) & (5) \\
\hline $\begin{array}{l}\text { 8. When in a group of people, I have trouble } \\
\text { thinking of the right things to talk about. }\end{array}$ & (1) & (2) & (3) & (4) & (5) \\
\hline $\begin{array}{l}\text { 9. I always seem to know what other peoples' } \\
\text { true feelings are no matter how hard they try } \\
\text { to conceal them. }\end{array}$ & (1) & (2) & (3) & (4) & (5) \\
\hline $\begin{array}{l}\text { 10. I am very good at maintaining a calm exterior } \\
\text { even if I am upset. }\end{array}$ & (1) & (2) & (3) & (4) & (5) \\
\hline $\begin{array}{l}\text { 11. If the situation calls for it, I am comfortable } \\
\text { with introducing myself to strangers. }\end{array}$ & (1) & (2) & (3) & (4) & (5) \\
\hline $\begin{array}{l}\text { 12. I can be strongly affected by someone smiling } \\
\text { or frowning at me. }\end{array}$ & (1) & (2) & (3) & (4) & (5) \\
\hline $\begin{array}{l}\text { 13. I would feel uncomfortable at a social event } \\
\text { attended by a lot of very important people. }\end{array}$ & (1) & (2) & (3) & (4) & (5) \\
\hline $\begin{array}{l}\text { 14. I am able to make interesting conversation } \\
\text { with others. }\end{array}$ & (1) & (2) & (3) & (4) & (5) \\
\hline $\begin{array}{l}\text { 15. I can instantly identify someone who is } \\
\text { insincere. }\end{array}$ & (1) & (2) & (3) & (4) & (5) \\
\hline $\begin{array}{l}\text { 16. While I may be nervous on the inside, I can } \\
\text { disguise it very well from others. }\end{array}$ & (1) & (2) & (3) & (4) & (5) \\
\hline $\begin{array}{l}\text { 17. At social events I enjoy talking to a lot of } \\
\text { different people. }\end{array}$ & (1) & (2) & (3) & (4) & (5) \\
\hline 18. It is very important that other people like me. & (1) & (2) & (3) & (4) & (5) \\
\hline $\begin{array}{l}\text { 19. If the situation calls for it, I can be an effective } \\
\text { group leader. }\end{array}$ & (1) & (2) & (3) & (4) & (5) \\
\hline 20. I am a sensitive, understanding person. & (1) & (2) & (3) & (4) & (5) \\
\hline
\end{tabular}




$\begin{array}{ccccc}\text { Not At } & \text { A Little } & \text { Like } & \begin{array}{c}\text { Very } \\ \text { Much }\end{array} & \text { Exactly } \\ \text { All Like } & \text { Like Me } & \text { Me } & \text { Like Me } & \text { Like Me } \\ \text { Me } & & & \end{array}$

21. I enjoy going to large social events and meeting new people.

(1) (2) (4)

(5)

22. I'm generally concerned about the impression I'm making on others.

(1) (2) (3)

(5)

23. I can easily adjust to being in just about any social situation.

(1) (2) (3) (4)


Finally, we would like to ask you some questions about yourself. You will never be personally identified in this research project or in any presentation or publication.

1. Which of the following best describes your

current situation?

$\odot \quad$ I live and work in a host country, but will return to my home country or will move to another international destination and retain my home country citizenship (expatriate).

๑) I live in a host country, and may stay for more than two years or apply for permanent residence (immigrant).

$\odot \quad$ I live and study in a host country, but will return to my home country or will move to another international destination and retain my home country citizenship (international student).

$\approx \quad$ I live in a host country due to war, violence, or persecution in my home country of citizenship and cannot or do not want to return (refugee).

$\circledast \quad$ Other, please describe:

2. What is your nationality?

3. How would you describe your

ethnic identity (e.g., Malay, Samoan)?

4. In which country were you born?

5. In which country have you lived most of your

life?

6. In which country do you currently live?

7. How long have you been in your host country?

months

8. What is your first language?

9. How would you describe your overall host language proficiency?

Poor Below Average Average

(1) (2)

(3)

Above Average

(4)

Excellent Native Speaker

10. What other languages do you speak?

Language 1:

Language 2 :

Language 3:

11. Gender:

Female

$\circledast$

13. How long are you planning to

stay in your host country?

1 Year Or Less

Between 1-3 Years

3 Years Or More

Permanently

$\circledast$

$\odot$

$\circledast$

(0) (0)

(1) (1)

(2) (2)

(3) (3)

(4) (4)

(5) (5)

(6) (6)

(7) (7)

(8) (8)

(9) (9)

๑ 


\section{VICTORIA UNIVERSITY OF WELLINGTON \\ Te Whare Wananga o te Upoko o te Ika a Maui}

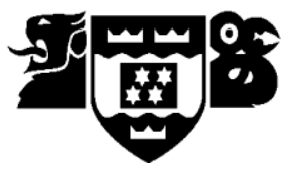

Debrief Sheet

Thank you for participating in my study. This research aims to develop a revised measure of sociocultural adaptation, which is a term used to describe the different skills an individual uses to live successfully in a new culture.

Previous research has shown that gaining specific skills is an important part of successfully engaging in and adapting to a new culture. These skills involve how well an individual interacts with others, speaks and understands the host country language, and the steps individuals take to involve themselves in their new community. The measure we are revising examines many different types of skills individuals use while living in a new culture. A portion of this study also involves comparing the measure to other similar questionnaires. I would like to make the new measure accessible to anyone who is interested in researching or learning more about adaptation in the hopes that the process of how a person adjusts to living in a new culture is more widely understood.

Overall, this research is significant because it provides us with a way to study different aspects of adaptation. Also, this research may help future researchers more accurately examine how people behave in and adjust to new cultures. Ultimately, gaining a fuller understanding of how members of our multicultural societies adapt to living in a different country is important for all of us, given the ever-increasing amount of students, expatriates, immigrants, and refugees moving internationally between nations across the globe.

Thank you again for participating in this research.

Jessie Wilson 


\section{VICTORIA UNIVERSITY OF WELLINGTON \\ Te Whare Wananga o te Upoko o te Ika a Maui}

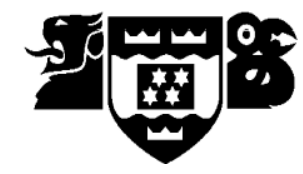

\section{Lucky Draw Contact Information Sheet}

In order to participate in the lucky draw, please complete the following information. This information is not linked to your previous survey responses in any way.

You will be contacted via e-mail only if you are the winner of the draw. Further information about your prize will be given to you at that time.

Name:

Postal Address Line 1:

Postal Address Line 2:

E-mail address:

Please indicate your preferred prize*:

1) iPod Nano (8gb)

*an iPod Nano is the default

2) $\$ 250$ gift voucher for Dick Smith Electronics

prize if the winner lives

3) $\$ 250$ gift voucher to the Warehouse

outside of New Zealand

4) $\$ 250$ gift voucher to New World supermarket 


\section{VICTORIA UNIVERSITY OF WELLINGTON \\ Te Whare Wananga o te Upoko o te Ika a Maui}

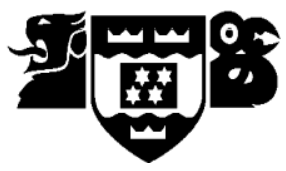

E-mail Request

To Whom It May Concern:

I am a PhD candidate at the Victoria University of Wellington, New Zealand researching how individuals experience different cultures. As part of my research, I have developed a measure to assess how a person adapts to living in a new cultural environment. The research I am conducting has been approved by the Victoria University School of Psychology Human Ethics Committee.

If possible, I would like to provide the members of your organization with some information about my online survey and a link to the survey (see below). I could post this information myself on your listserv or through an e-mail to members. Or, if you prefer, the information and link I've provided below could be used to notify your organization's members in whatever manner you choose.

Please contact me with any questions you have about this research, and thank you in advance for your time and consideration.

Kind Regards,

Jessie Wilson

Jessie.wilson@vuw.ac.nz

Centre for Applied Cross-Cultural Research

School of Psychology

Te Kura Mătai Hinengaro

Victoria University of Wellington

PO Box 600

Wellington, New Zealand

Information and link to survey:

\section{Opportunity to Participate in a Psychology Study and a Chance to Win a Free iPod Nano or \$250 Gift Voucher}

I am a PhD candidate at Victoria University conducting an online study about how people adapt to living in a new culture.

The survey is both voluntary and completely anonymous. If you are interested in participating, there is a link below that provides more information about the research, how you can participate, and the survey items. The survey will take no more than 60 minutes for you to complete.

If you complete the survey, you can go into a lucky draw for an iPod Nano (8gb) or a $\$ 250$ gift voucher from Dick Smith, the Warehouse, or New World (an iPod Nano is the default prize if the winner lives outside of New Zealand).

To participate, you must be an international student or short-term migrant who is not currently living in your home country, and who has lived in your new host country of residence for less than two years. Also, you cannot participate in the survey if you are under 16 years of age.

Please contact me at jessie.wilson@vuw.ac.nz if you have further questions about this research. 
Appendix E. Study 3 Participant Demographics $(N=185)$

\begin{tabular}{|c|c|c|}
\hline & $\mathrm{N}$ & $\%$ \\
\hline Participant Total & 185 & \\
\hline \multicolumn{3}{|l|}{ Gender } \\
\hline Female & 133 & 73.10 \\
\hline Male & 49 & 26.50 \\
\hline Missing Data & 3 & 1.60 \\
\hline \multicolumn{3}{|l|}{ Age $(M=39.20)$} \\
\hline 20 and younger & 2 & 1.10 \\
\hline $21-30$ & 26 & 14.10 \\
\hline $31-40$ & 74 & 4.00 \\
\hline $41-50$ & 50 & 27.00 \\
\hline $51-60$ & 23 & 12.40 \\
\hline 60 and older & 1 & .50 \\
\hline Missing Data & 9 & 4.90 \\
\hline \multicolumn{3}{|l|}{ Highest Completed Qualifications } \\
\hline Secondary School & 11 & 5.90 \\
\hline Post Secondary Certificate/Diploma & 17 & 9.20 \\
\hline Vocational Qualification/Trade Certificate & 13 & 7.00 \\
\hline Tertiary Degree & 64 & 34.60 \\
\hline Postgraduate Degree & 76 & 41.10 \\
\hline Missing Data & 4 & 2.20 \\
\hline \multicolumn{3}{|l|}{ English Language Proficiency } \\
\hline Below Average & 2 & 1.10 \\
\hline Average & 4 & 2.20 \\
\hline Above Average & 30 & 16.20 \\
\hline Excellent & 51 & 27.60 \\
\hline Native Speaker & 94 & 5.80 \\
\hline Missing Data & 4 & 2.20 \\
\hline \multicolumn{3}{|l|}{ Nationality } \\
\hline Algerian & 1 & .50 \\
\hline American & 21 & 11.40 \\
\hline Argentinian & 1 & .50 \\
\hline Australian & 1 & .50 \\
\hline Austrian & 1 & .50 \\
\hline Belgian & 1 & .50 \\
\hline Bengali & 1 & .50 \\
\hline Brazilian & 2 & 1.10 \\
\hline British & 49 & 26.50 \\
\hline Burmese & 1 & .50 \\
\hline Chilean & 1 & .50 \\
\hline Chinese & 1 & .50 \\
\hline Colombian & 2 & 1.10 \\
\hline Croatian & 1 & .50 \\
\hline Danish & 1 & .50 \\
\hline Dual Nationality & 9 & 4.90 \\
\hline Dutch & 7 & 3.80 \\
\hline
\end{tabular}


Egyptian

Fijian

1.60

Filipino

9.20

Finnish

.50

German

2.70

Hong Kong

.50

Hungarian

1.10

Indian

3.80

Iranian

Irish

.50

Italian

Kiribati

Korean

Malaysian

Nepali

Norwegian

Pakistani

Palestinian

Romanian

Russian

Scottish

South African

.10

.50

.50

2.20

2.70

.50

.50

.50

.50

.50

1.60

1.10

Sri Lankan

Swiss

5.90

Taiwanese

Thai

Vietnamese

Missing Data

.50

.50

.50

.50

3.80

Ethnicity

European

62.70

Pacific Peoples

1.60

Asian

3

24.90

Middle Eastern/Latin American/African

7.60

Other Ethnicity

Missing Data

.50

2.70

New Zealand Permanent Resident

Yes

75.10

No

Missing Data

Years in New Zealand

1 year or less

1 to 2 years

2 to 3 years

3 to 4 years

13.50

4 to 5 years

15.70

Missing Data

Employment Status

8

18.90

Not Employed

Employed Part-Time 
Student, Employed Part-Time

Student, Employed Full-Time

1.10

Employed Full-Time

8

Missing Data

8

48.10

4.30 


\section{VICTORIA UNIVERSITY OF WELLINGTON}

Te Whare Wananga o te Upoko o te Ika a Maui

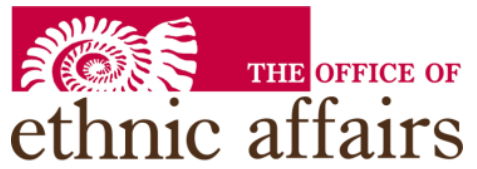

Te Tari Matawaka

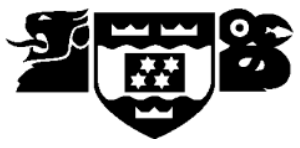

Introduction

Welcome to a research project organized through Victoria University's Centre for Applied CrossCultural Research in collaboration with the Office of Ethnic Affairs, and conducted by PhD candidate Jessie Wilson under the supervision of Professor Colleen Ward. We would like to invite you to participate in a study about your experiences in New Zealand and what motivated your move here. This project has been approved by the university's ethics committee.

\section{To complete the following survey, you must: -have been born outside of New Zealand -be 16 years of age and older, and -have been living in New Zealand for 5 years or less.}

Your participation in the study will involve completing an anonymous questionnaire. The survey takes less than 30 minutes to complete and is entirely voluntary. It includes questions such as "How competent are you at building and maintaining relationships?", "What was the main reason that motivated your move to New Zealand?", and "Have you ever experienced less respect than others?".

You do not have to take part in the study. If you do agree to participate, you are free to stop at any time without having to give a reason.

If you complete the questionnaire, it will be understood that you have consented to participate in the project, and also consent to publication of the results with the understanding that your anonymity will be preserved.

The data collected for this study will remain with us, be stored securely in the School of Psychology at Victoria University for at least five years, and be shared only with competent professionals on a case-by-case basis.

Preliminary results from the study will be posted on the Centre for Applied Cross-Cultural Research website (www.vuw.ac.nz/cacr) in May 2011. Or, you may provide your e-mail address below and a summary of the results will be e-mailed to you.

If you have any queries about the project, or if you would like to receive more information, please do not hesitate to contact us. We are happy to answer your questions. Thank you very much for your help and cooperation!

Kind Regards,

Jessie Wilson, PhD Candidate (jessie.wilson@vuw.ac.nz or 04463 5311)

Colleen Ward, Professor, School of Psychology (colleen.ward@vuw.ac.nz)

If you are interested in receiving a summary of results from the study, please enter your e-mail address below and return with your survey. Your e-mail address will not be used in any other way, and will not be connected to your survey responses. 
VICTORIA UNIVERSITY OF WELLINGTON

Te Whare Wananga o te Upoko o te Ika a Maui

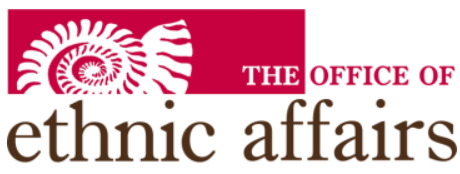

Te Tari Matawaka

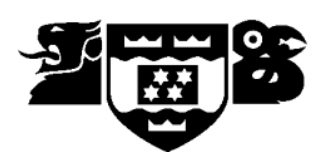

Instructions

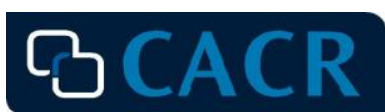

CENTRE FOR APPLIED CROSS-CULTURAL RESEARCH Te Pae Rangahau Tauhōkai Ahurea

Most of this survey will be recorded using a digital scanner. Please do not use a pencil. Instead, mark your answers carefully using a black or blue pen in this way:

\begin{tabular}{|l|lll|l|}
\hline 1. Statement & (1) & (2) & & \\
& & & & \\
\hline
\end{tabular}

If you need to change an answer, please cross out the incorrect one this way:

\begin{tabular}{|l|ll|}
\hline 1. Statement & (1) & (2)
\end{tabular}

On the following pages, we will ask some questions about yourself and how you behave in particular situations. Please answer each question using the provided rating scales. For example, fill in 2 if you eat fruit only very rarely. Please remember, there are no right or wrong answers.

\begin{tabular}{|l|ccccccc|}
\hline & Never & Very Rarely & Rarely & Sometimes & Frequently & $\begin{array}{c}\text { Very } \\
\text { Frequently }\end{array}$ & Always \\
\cline { 2 - 7 } 1. I eat fruit. & (1) & (2) & (3) & (4) & (5) & (6) & (7) \\
\hline
\end{tabular}


1. What was the main reason that motivated your move to New Zealand?

2. Read each statement and select the response that best describes your capabilities. Select the answer that BEST describes you as you really are ( 1 = Strongly disagree; 7 = Strongly agree).

\begin{tabular}{|l}
$\begin{array}{l}\text { 1. I enjoy interacting with } \\
\text { people from different } \\
\text { cultures. }\end{array}$ \\
$\begin{array}{l}\text { 2trongly } \\
\text { disagree }\end{array}$ \\
$\begin{array}{l}\text { I enjoy living in cultures that } \\
\text { are unfamiliar to me. }\end{array}$ \\
3. I am confident that I can \\
$\begin{array}{l}\text { socialize with locals in a } \\
\text { culture that is unfamiliar to } \\
\text { me. }\end{array}$ \\
$\begin{array}{l}\text { 4. I am confident that I can get } \\
\text { accustomed to the shopping } \\
\text { conditions in a different } \\
\text { culture. }\end{array}$ \\
$\begin{array}{l}\text { 5. I am sure I can deal with the } \\
\text { stresses of adjusting to a } \\
\text { culture that is new to me. }\end{array}$
\end{tabular}


3. There might have been different reasons why you were motivated to move to New Zealand.

Please indicate to what extent each of the following reasons to move to New Zealand applies to you. Some statements may seem very similar to each other, but despite this please rate all of them.

\begin{tabular}{|c|c|c|c|c|c|}
\hline & $\begin{array}{l}\text { Not at all } \\
\text { because of } \\
\text { this reason }\end{array}$ & & $\begin{array}{l}\text { Somewhat } \\
\text { because of } \\
\text { this reason }\end{array}$ & & $\begin{array}{l}\text { Completely } \\
\text { because of } \\
\text { this reason }\end{array}$ \\
\hline $\begin{array}{l}\text { 1. I moved to New Zealand because I } \\
\text { thought it would be fun and } \\
\text { interesting. }\end{array}$ & (1) & (2) & (3) & (4) & (5) \\
\hline $\begin{array}{l}\text { 2. I moved to New Zealand because it } \\
\text { was personally important to me. }\end{array}$ & (1) & (2) & (3) & (4) & (5) \\
\hline $\begin{array}{l}\text { 3. I moved to New Zealand because other } \\
\text { people (relatives and friends) expected } \\
\text { me to do this. }\end{array}$ & (1) & (2) & (3) & (4) & (5) \\
\hline $\begin{array}{l}\text { 4. I moved to New Zealand so that other } \\
\text { people would be proud of me. }\end{array}$ & (1) & (2) & (3) & (4) & (5) \\
\hline $\begin{array}{l}\text { 5. I moved to New Zealand because I } \\
\text { wanted to avoid the shame and guilt } \\
\text { of not doing this. }\end{array}$ & (1) & (2) & (3) & (4) & (5) \\
\hline $\begin{array}{l}\text { 6. I moved to New Zealand because I } \\
\text { thought I would enjoy it. }\end{array}$ & (1) & (2) & (3) & (4) & (5) \\
\hline $\begin{array}{l}\text { 7. I moved to New Zealand because this } \\
\text { is what I really want to do with my life. }\end{array}$ & (1) & (2) & (3) & (4) & (5) \\
\hline $\begin{array}{l}\text { 8. I moved to New Zealand because } \\
\text { others (spouse, family, friends) were } \\
\text { pushing me to do this. }\end{array}$ & (1) & (2) & (3) & (4) & (5) \\
\hline $\begin{array}{l}\text { 9. I moved to New Zealand because I } \\
\text { expected to get respect and } \\
\text { recognition from others for doing so. }\end{array}$ & (1) & (2) & (3) & (4) & (5) \\
\hline $\begin{array}{l}\text { 10. I moved to New Zealand because it is a } \\
\text { prestigious thing to do. }\end{array}$ & (1) & (2) & (3) & (4) & (5) \\
\hline $\begin{array}{l}\text { 11. I moved to New Zealand so that other } \\
\text { people would approve of me. }\end{array}$ & (1) & (2) & (3) & (4) & (5) \\
\hline
\end{tabular}




$\begin{array}{ccc}\begin{array}{c}\text { Not at all } \\ \text { because of }\end{array} & \begin{array}{c}\text { Somewhat } \\ \text { because of }\end{array} & \begin{array}{c}\text { Completely } \\ \text { because of } \\ \text { this }\end{array} \\ \text { reason } & \text { this reason } & \text { this reason }\end{array}$

12. I moved to New Zealand because I thought it would be an exciting thing to do.

(1) (2) (3) (4)

(5)

13. I moved to New Zealand because others (e.g., spouses, family) forced me to do this.

14. I moved to New Zealand because it was one of my life goals.

(1)

(2)

(3)

(4)

(5)

15. I moved to New Zealand because it was of great personal value to me.

(1) (2) (3) (4)

(5)

16. I moved to New Zealand because I would have been criticized if I did not.

(1) (2) (3) (4)

(5)

17. I moved to New Zealand because it was an opportunity that I highly valued.

18. I moved to New Zealand because I was highly interested in doing this.

(1) (2) (4)

(5)

19. I moved to New Zealand because that's what I was expected to do.

(1) (2) (3) (4)

(5)

20. I moved to New Zealand because I felt that I was forced to do so.

(1) (2) (4)

(5) 
4. The following section lists specific reasons why you may have been motivated to move to New Zealand. Similar to the previous section, please indicate to what extent each of the reasons applies to you personally.

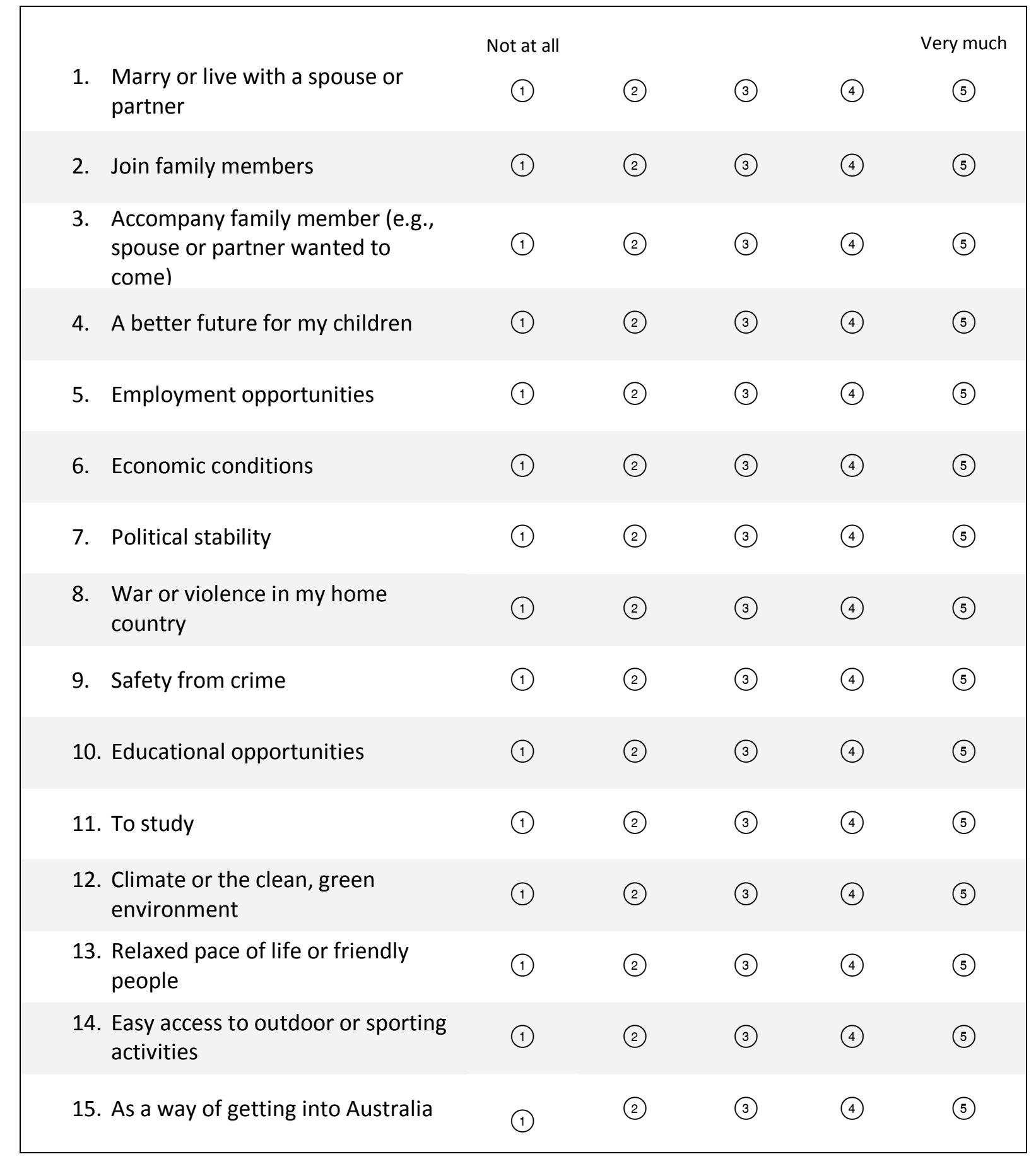


5. Please indicate how frequently you have experienced the following forms of personal mistreatment in your day-to-day life in New Zealand.

Never Rarely Sometimes Often

1. You have experienced less respect than others.

(1)

(2) (3)

(4)

2. You received poorer service than others in restaurants or stores.

(1) (3)

(4)

3. People acted as if you were unintelligent.

(1)

(2)

(3)

(4)

4. People acted as if they were better than you.

(1)

(2)

(3)

(4)

5. People acted as if they were afraid of you.

(1)

(2)

(3)

(4)

6. People acted as if they thought you were dishonest.

(1)

(2)

(3)

(4)

7. You were called names or insulted.

(1)

(2)

(3)

(4)

8. You were threatened or harassed.

(2)

(3) 
6. Listed below are a number of statements concerning personal attitudes and traits. Read each item and decide whether the statement is True or False as it pertains to you personally.

True

False

1. It is sometimes hard for me to go on with my work if I am not encouraged.

(T)

2. I sometimes feel resentful when I don't get my way.

(T)

3. On a few occasions, I have given up doing something because I thought too little of my ability.

4. There have been times when I felt like rebelling against people in authority even though I knew they were right.

(T)

5. No matter who I'm talking to, I'm always a good listener.

(T)

(F)

6. There have been occasions when I took advantage of someone.

(T)

7. I'm always willing to admit it when I make a mistake.

(

8. I sometimes try to get even rather than forgive and forget.

(

9. I am always polite, even to people who are disagreeable.

(T)

10. I have never been upset when people expressed ideas very different from my own.

(T)

(F)

11. There have been times when I was quite jealous of the good fortune of others.

(

(F)

12. I am sometimes irritated by people who ask favors of me.

(T)

(F)

13. I have never deliberately said something that hurt someone's feelings. 
7. Living in a different culture often involves learning new skills and behaviors. Thinking about life in New Zealand, please rate your competence (e.g., skill) at each the following behaviors ( $1=$ Not at all competent; 5 = Extremely competent).

\section{Not at all}

competent

Extremely competent

1. Building and maintaining relationships.

(1)

(2)

(3)

(4)

(5)

2. Managing my work responsibilities.

(1)

(2)

(3)

(4)

(5)

3. Interacting at social events.

(1)

(2)

(3)

(4)

(5)

4. Maintaining my hobbies and interests.

(1) (2)

(3)

(4)

(5)

5. Adapting to the noise level in my neighborhood.

(1) (2) (3)

(5)

6. Accurately interpreting and responding to other people's

(1) (2)

(2) (3)

(4)

(5) gestures and facial expressions.

7. Working effectively with other work colleagues.

(1) (2) (4)

(5)

8. Obtaining community services I require.

(1)

(2) (3)

(4)

(5)

9. Adapting to the population density

(1)

(2)

(3)

(4)

(5)

10. Understanding and speaking English.

(1)

(2)

(3)

(4)

(5)

11. Varying the rate of my speaking in a culturally appropriate manner.

(1)

(2)

(3)

(4)

(5)

12. Gaining feedback from other work colleagues to help improve

(1) (2)

(3)

(4)

(5) my performance. 
13. Accurately interpreting and responding to other people's

(1)

(2)

(3)

(4)

(5) emotions.

14. Attending or participating in community activities.

(1)

(2)

(3)

(4)

(5)

15. Finding my way around.

(1)

(2)

(3)

(4)

(5)

16. Interacting with members of the opposite sex.

(1)

(2)

(3)

(4)

(5)

17. Expressing my ideas to other work colleagues in a culturally

(1)

(2)

(3)

(4)

(5) appropriate manner.

18. Dealing with the bureaucracy.

(1)

(2)

(3)

(4)

(5)

19. Adapting to the pace of life.

(1)

(2)

(3)

(4)

(5)

20. Reading and writing English.

(1)

(2)

(3)

(4)

(5)

21. Changing my behavior to suit social norms, rules, attitudes, beliefs, and (1)

(2)

(3)

(4) customs. 
8. Please indicate how strongly you agree (5) or disagree (1) with each statement:

\section{Strongly}

Disagree
Strongly

Agree

1. In most ways my life is close to my ideal.

(1)

(2)

(3)

(4)

(5)

2. The conditions of my life are excellent.

(1)

(2)

(3)

(4)

(5)

3. I am satisfied with my life.

(1)

(2)

(3)

(4)

(5)

4. So far I have got the important things I want in life.

(1) (2) (4) (5)

5. If I could live my life over, I would change almost nothing.

(2)

(3)

(4)

(5) 
9. Please indicate how frequently you experience the following ( $1=$ a little of the time; $4=$ most of the time).

\begin{tabular}{|c|c|c|c|c|}
\hline & $\begin{array}{l}\text { A little of } \\
\text { the time }\end{array}$ & $\begin{array}{c}\text { Some of the } \\
\text { time }\end{array}$ & $\begin{array}{l}\text { A good part } \\
\text { of the time }\end{array}$ & $\begin{array}{c}\text { Most of the } \\
\text { time }\end{array}$ \\
\hline 1. I feel sad. & (1) & (2) & (3) & (4) \\
\hline 2. Morning is when I feel best. & (1) & (2) & (3) & (4) \\
\hline 3. I have crying spells. & (1) & (2) & (3) & (4) \\
\hline 4. I have trouble sleeping at night. & (1) & (2) & (3) & (4) \\
\hline 5. I eat as much as I used to. & (1) & (2) & (3) & (4) \\
\hline 6. I notice that my weight has changed. & (1) & (2) & (3) & (4) \\
\hline 7. I have trouble with constipation. & (1) & (2) & (3) & (4) \\
\hline 8. My heart beats faster than usual. & (1) & (2) & (3) & (4) \\
\hline 9. I get tired for no reason. & (1) & (2) & (3) & (4) \\
\hline 10. My mind is as clear as it used to be. & (1) & (2) & (3) & (4) \\
\hline 11. I find it easy to do things I used to do. & (1) & (2) & (3) & (4) \\
\hline 12. I am restless and cannot keep still. & (1) & (2) & (3) & (4) \\
\hline 13. I am hopeful about the future. & (1) & (2) & (3) & (4) \\
\hline
\end{tabular}




$\begin{array}{ccc}\text { A little of } & \text { Some of the } & \text { A good part } \\ \text { the time } & \text { time } & \text { Most of the } \\ \text { of the time } & \text { time }\end{array}$

14. I feel more irritable than I used to be.

(1)

(2)

(3)

(4)

15. I find it easy to make decisions.

(1)

(2)

(3)

(4)

16. I feel that I am useful and needed.

(1)

(2)

(3)

(4)

17. My life is meaningful.

(1)

(2)

(3)

(4)

18. I feel that others would be better off if I were dead.

(1) (3)

(4)

19. I still enjoy the things I used to.

(1)

(2)

(3)

(4) 
10. Please indicate your current employment status:

\begin{tabular}{|cccccc|}
\hline Not employed & $\begin{array}{c}\text { Employed part- } \\
\text { time (less than } 30 \\
\text { hours per week) }\end{array}$ & $\begin{array}{c}\text { Student, not } \\
\text { employed }\end{array}$ & $\begin{array}{c}\text { Student, } \\
\text { employed part- } \\
\text { time (less than } 30 \\
\text { hours per week) }\end{array}$ & $\begin{array}{c}\text { Student, } \\
\text { employed full- } \\
\text { time (more than } \\
30 \text { hours per } \\
\text { week) }\end{array}$ & $\begin{array}{c}\text { Employed full- } \\
\text { time (more than } \\
30 \text { hours per } \\
\text { week }\end{array}$ \\
(1) & (2) & (3) & (4) & (5)
\end{tabular}

11. How would you rate your chances of quitting your current job:

Very low

Very high

1. In the next three months?

(1)

(2)

(3)

(4)

(5)

(6)

(7)

2. In the next year?

(1)

(2)

(3)

(4)

(5)

(6)

(7)

3. In the next two years?

(1) (2)

(3)

(4)

(5)

(6)

(7)

4. In the next 5 years?

(1)

(2)

(3)

(4)

(5)

(6)

(7) 
12. Please indicate the extent to which you disagree or agree with the following statements.

$\begin{aligned} & \text { 1. I was hired according to my educational } \\ & \text { qualifications. }\end{aligned}$
$\begin{aligned} & \text { 2. My educational background is recognized in } \mathrm{my} \\ & \text { current position. }\end{aligned}$
$\begin{aligned} & \text { 3. } \\ & \text { My educational qualifications are recognized in } \\ & \text { my job. }\end{aligned}$
$\begin{aligned} & \text { 4. } \\ & \text { My job uses the skills I gained from previous } \\ & \text { work experience. }\end{aligned}$


13. Please indicate your level of agreement for each of the following statements.

\begin{tabular}{|c|c|c|c|c|c|c|c|}
\hline & $\begin{array}{l}\text { Strongly } \\
\text { disagree }\end{array}$ & & & & & & $\begin{array}{l}\text { Strongly } \\
\text { agree }\end{array}$ \\
\hline $\begin{array}{l}\text { of effort beyond that normally } \\
\text { expected in order to help this } \\
\text { organization be successful. }\end{array}$ & (1) & (2) & (3) & (4) & (5) & (6) & (7) \\
\hline $\begin{array}{l}\text { 2. I tell my friends that this is a } \\
\text { great organization to work for. }\end{array}$ & (1) & (2) & (3) & (4) & (5) & (6) & (7) \\
\hline $\begin{array}{l}\text { 3. I would accept almost any type } \\
\text { of job assignment in order to } \\
\text { keep working for this } \\
\text { organization. }\end{array}$ & (1) & (2) & (3) & (4) & (5) & (6) & (7) \\
\hline $\begin{array}{l}\text { 4. I find that my values and the } \\
\text { organization's values are very } \\
\text { similar. }\end{array}$ & (1) & (2) & (3) & (4) & (5) & (6) & (7) \\
\hline $\begin{array}{l}\text { 5. I am proud to tell others that I } \\
\text { am part of this organization. }\end{array}$ & (1) & (2) & (3) & (4) & (5) & (6) & (7) \\
\hline $\begin{array}{l}\text { 6. This organization really inspires } \\
\text { the very best in me in the way of } \\
\text { job performance. }\end{array}$ & (1) & (2) & (3) & (4) & (5) & (6) & (7) \\
\hline $\begin{array}{l}\text { 7. I am extremely glad that I chose } \\
\text { this organization to work for } \\
\text { over others I was considering at } \\
\text { the time I joined. }\end{array}$ & (1) & (2) & (3) & (4) & (5) & (6) & (7) \\
\hline $\begin{array}{l}\text { 8. I really care about the fate of } \\
\text { this organization. }\end{array}$ & (1) & (2) & (3) & (4) & (5) & (6) & (7) \\
\hline $\begin{array}{l}\text { 9. For me this is the best of all } \\
\text { possible organizations to work } \\
\text { for. }\end{array}$ & (1) & (2) & (3) & (4) & (5) & (6) & (7) \\
\hline
\end{tabular}


14. Please indicate how strongly you agree (5) or disagree (1) with each statement:

\begin{tabular}{|c|c|c|c|c|c|}
\hline & $\begin{array}{l}\text { Strongly } \\
\text { Disagree }\end{array}$ & & & & $\begin{array}{l}\text { Strongly } \\
\text { Agree }\end{array}$ \\
\hline $\begin{array}{l}\text { corresponds closely with what I want in } \\
\text { life. }\end{array}$ & (1) & (2) & (3) & (4) & (5) \\
\hline $\begin{array}{l}\text { 2. The conditions under which I do my } \\
\text { work are excellent. }\end{array}$ & (1) & (2) & (3) & (4) & (5) \\
\hline 3. I am satisfied with the type of work I do. & (1) & (2) & (3) & (4) & (5) \\
\hline $\begin{array}{l}\text { 4. So far I have obtained the important } \\
\text { things I want from my work. }\end{array}$ & (1) & (2) & (3) & (4) & (5) \\
\hline $\begin{array}{l}\text { 5. If I could change anything at work, I } \\
\text { would change almost nothing. }\end{array}$ & (1) & (2) & (3) & (4) & (5) \\
\hline
\end{tabular}


15. We would like to ask you some questions about yourself. You will never be personally identified in this research project or in any publication.

1. How would you describe your ethnic identity (e.g., Malay, Samoan)?

2. In which country were you born?

3. What is your nationality?

4. Do you currently live in New

4. Zealand?

Yes $\odot$ No $\odot$

5. How long have you lived in New Zealand? year(s) $\operatorname{month}(\mathrm{s})$

6. How would you describe your overall English language proficiency (reading, writing, understanding, and speaking) when you first came to New Zealand?

Poor Below Average $\quad$ Average $\quad$ Above Average $\quad$ Excellent $\quad \begin{gathered}\text { Native } \\ \text { Speaker }\end{gathered}$
(1)
(2)
(3)
(4)
(5)

(6)

7. How would you describe your overall English language proficiency now?

$\begin{array}{lllllr}\text { Poor } & \text { Below Average } & \text { Average } & \text { Above Average } & \text { Excellent } & \begin{array}{r}\text { Native } \\ \text { Speaker }\end{array} \\ \text { (1) } & \text { (2) } & \text { (3) } & \text { (4) } & \text { (5) } & \text { (6) }\end{array}$

8. What is the highest educational qualification you have achieved?

\begin{tabular}{|c|c|c|c|c|}
\hline $\begin{array}{l}\text { Primary school } \\
\text { qualification }\end{array}$ & $\begin{array}{l}\text { Secondary school } \\
\text { qualification }\end{array}$ & $\begin{array}{l}\text { Post-secondary } \\
\text { certificate/ } \\
\text { Diploma }\end{array}$ & $\begin{array}{c}\text { Vocational } \\
\text { qualification/ } \\
\text { Trade certificate }\end{array}$ & Tertiary degree \\
\hline
\end{tabular}
(1)
(2)
(3)
(4)
(5)
(6)

9. Gender: $\quad$ Female $\circledast$ Male $\circledast$ 10. Age:

11. How long do you intend to stay in New Zealand?

1 year or less

Between 1-3 years

Between 3-5 years
( 5 years or more

$\odot \quad$ I do not intend on leaving NZ

$\circledast$ $\circledast$

๑ 
12. If you intend to leave New Zealand, will you be returning to your home country or moving to another country?
Home country
$\odot$
Other country
$\circledast$
Don't know

$\odot$

13. Name of other country you plan to move to:

14. Are you a permanent resident of New Zealand?
Yes
$\circledast$
No
$\circledast$

15. If you are not a permanent resident, what kind of visa or permit do you currently hold?

Business visa

$\circledast$

Essential skills category

$\circledast$

Family visa

$\circledast$

Long-term business visa

$\odot$

Pacific access category

$\circledast$

Recognised seasonal employer scheme

$\star$

Refugee family support category

$\star$

Samoan quota scheme

Æ

Skilled migration category (e.g., Silver Fern visa)

$\star$

Student visa/permit

$\star$

Tourist visa

た

Visitor's permit

$\star$

Work to residence visa

$\star$

Work visa/permit

$\star$

Working holiday visa 
Don't know

$\circledast$

None of these

$\circledast$

16. If you answered "none of these", please describe your current visa or permit situation:

17. Are you intending to apply for New Zealand citizenship? $\quad$ Yes $\odot$ No $\odot$

18. Which region of New Zealand do you currently live in?

\begin{tabular}{|c|c|c|}
\hline Northland & $\circledast$ & Wellington \\
\hline Auckland & $\circledast$ & Tasman \\
\hline Waikato & $\circledast$ & Nelson \\
\hline Bay of Plenty & $\odot$ & Malborough \\
\hline Gisborne & $\odot$ & West Coast \\
\hline Hawke's Bay & $\circledast$ & Canterbury \\
\hline Taranaki & * & Otago \\
\hline Manawatu-Wanganui & $(*)$ & Southland \\
\hline
\end{tabular}

19. How did you find out about this survey? 


\section{Debrief}

Thank you for participating in our study. This research looks at your experiences as a migrant in New Zealand in terms of migration motivation, perceived discrimination, and adaptation. In particular, this survey examined three different aspects of migration motivation: 1) confidence with and enjoyment for experiencing different cultures; 2) relational, socio-economic, and career or educational reasons for migrating; and 3) type of motivation you experienced in moving to New Zealand (e.g., whether you were personally motivated or motivated by external factors). We are interested in determining how these different facets of motivation may relate to your potential experience of discrimination. Further, we are examining how both motivation and discrimination affect adaptation.

Adapting to a new country involves both psychological and behavioral factors. Psychologically, migrants living in a new culture can experience changes in mood, life and job satisfaction, and intentions to remain in or leave their host country. Migrants adapting to a different environment can also experience behavioral changes. This project utilizes a new measure of behavioral change known as sociocultural adaptation, which relates to the competencies you have developed in adapting to life in New Zealand. These competencies or skills include interpersonal communication, English language fluency, work behaviors, and community involvement.

Overall, we are hopeful that this research will further explain why some people are not as negatively affected by discrimination as others. This project may also allow future researchers to better examine how people behave in and adjust to new cultures. Ultimately, gaining a fuller understanding of how members of our multicultural society adapt to and experience life in a different country is important for all of us, given the continued increase of international migration and resulting ethnic diversity across nations.

Please place your completed survey in the enclosed stamped envelope along with your lucky draw information, survey feedback (next page), and lucky draw envelope and post before 1 May 2011.

Thank you again for participating in this research.

Kind Regards,

Jessie Wilson and Colleen Ward 


\section{Lucky Draw and Feedback}

As further thanks for your participation, we would like to offer you the opportunity to enter a lucky draw for a chance to win a $\$ 250$ gift voucher. If you are interested in entering the lucky draw, please complete the following information. This information will not be linked to your previous survey responses in any way.

If you are returning this page with your completed survey, please put it in the small envelope marked "Lucky Draw". Seal the lucky draw envelope, and post it along with your survey using the stamped envelope before 1 May 2011.

You will be contacted via e-mail only if you are the winner of the draw. Further information about your gift voucher will be given to you at that time.

Your name:

Street address:

Suburb:

City \& postal code:

Your e-mail address:

Please select your preferred \$250 gift voucher:

Dick Smith Electronics

New World Supermarket

The Warehouse

Please use the section below if you would like to comment on any part of the survey you have just completed or on the research project in general: 